NASA/TP-2009-213439

\title{
Integrity of the Plasma Magnetic Nozzle
}

Richard A. Gerwin

Los Alamos National Laboratory, Los Alamos, New Mexico 


\section{NASA STI Program . . . in Profile}

Since its founding, NASA has been dedicated to the advancement of aeronautics and space science. The NASA Scientific and Technical Information (STI) program plays a key part in helping NASA maintain this important role.

The NASA STI Program operates under the auspices of the Agency Chief Information Officer. It collects, organizes, provides for archiving, and disseminates NASA's STI. The NASA STI program provides access to the NASA Aeronautics and Space Database and its public interface, the NASA Technical Reports Server, thus providing one of the largest collections of aeronautical and space science STI in the world. Results are published in both non-NASA channels and by NASA in the NASA STI Report Series, which includes the following report types:

- TECHNICAL PUBLICATION. Reports of completed research or a major significant phase of research that present the results of NASA programs and include extensive data or theoretical analysis. Includes compilations of significant scientific and technical data and information deemed to be of continuing reference value. NASA counterpart of peer-reviewed formal professional papers but has less stringent limitations on manuscript length and extent of graphic presentations.

- TECHNICAL MEMORANDUM. Scientific and technical findings that are preliminary or of specialized interest, e.g., quick release reports, working papers, and bibliographies that contain minimal annotation. Does not contain extensive analysis.

- CONTRACTOR REPORT. Scientific and technical findings by NASA-sponsored contractors and grantees.
- CONFERENCE PUBLICATION. Collected papers from scientific and technical conferences, symposia, seminars, or other meetings sponsored or cosponsored by NASA.

- SPECIAL PUBLICATION. Scientific, technical, or historical information from NASA programs, projects, and missions, often concerned with subjects having substantial public interest.

- TECHNICAL TRANSLATION. Englishlanguage translations of foreign scientific and technical material pertinent to NASA's mission.

Specialized services also include creating custom thesauri, building customized databases, organizing and publishing research results.

For more information about the NASA STI program, see the following:

- Access the NASA STI program home page at http://www.sti.nasa.gov

- E-mail your question via the Internet to help@ sti.nasa.gov

- Fax your question to the NASA STI Help Desk at $443-757-5803$

- Telephone the NASA STI Help Desk at 443-757-5802

- Write to: NASA Center for AeroSpace Information (CASI) 7115 Standard Drive Hanover, MD 21076-1320 
NASA/TP-2009-213439

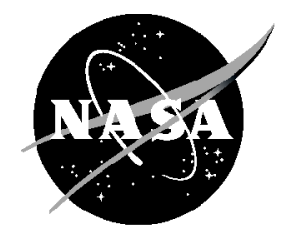

\section{Integrity of the Plasma Magnetic Nozzle}

Richard A. Gerwin

Los Alamos National Laboratory, Los Alamos, New Mexico

Prepared under C-32057-G

National Aeronautics and

Space Administration

Glenn Research Center Cleveland, Ohio 44135 


\section{Acknowledgments}

The author expresses gratitude to Dr. Peter J. Turchi and Dr. Dan Winske for their very helpful discussions. The author also wishes to thank the team of reviewers Dr. Ronald C. Davidson and Dr. Michael R. LaPointe for the time and effort they spent in reviewing and improving the manuscript. In addition, the author gratefully thanks Craig H. Williams and

Laura R. Becker for significantly improving the clarity of presentation of this multifaceted subject.

Level of Review: This material has been technically reviewed by three expert reviewers.

Available from

NASA Center for Aerospace Information

7115 Standard Drive

Hanover, MD 21076-1320
National Technical Information Service 5285 Port Royal Road Springfield, VA 22161

Available electronically at http://gltrs.grc.nasa.gov 


\section{Contents}

Summary

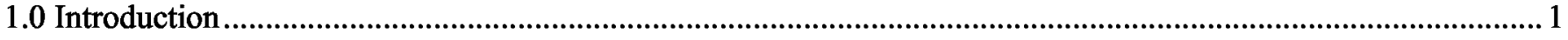

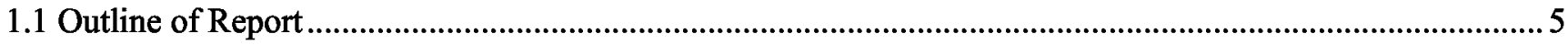

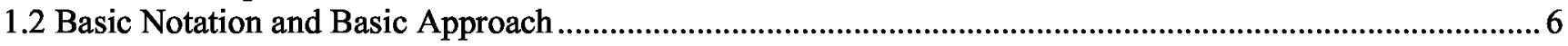

2.0 General Physics Description of Magnetic Nozzle........................................................................................... 6

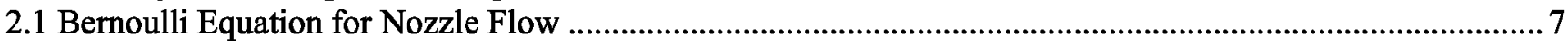

2.2 Diffusive Intermixing in Plasma-Field Interface ......................................................................................... 9

2.2.1 Diffusion of Wall-Confined Plasma...........................................................................................................

2.2.2 Diffusion of Magnetically Confined Plasma.............................................................................................. 10

2.2.3 Diffusion of Confining Magnetic Field............................................................................................... 13

2.3 Hall Effect, Plasma Rotation, and Electron Pressure in Ohm's Law .......................................................... 14

2.3.1 No Plasma Rotation, No Electron Pressure............................................................................ 15

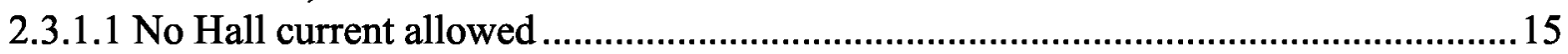

2.3.1.2 Hall current allowed (voltage shorted out)................................................................16

2.3.2 Plasma Rotation and Electron Pressure............................................................................................. 18

2.3.2.1 No Hall current allowed .................................................................................

2.3.2.2 Hall current allowed (voltage shorted out)..............................................................19

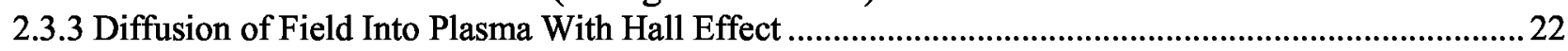

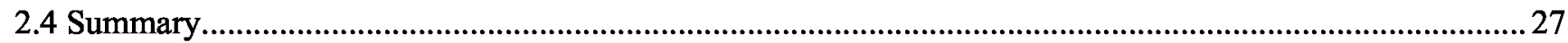

3.0 Resistivity From Gradient-Driven Microinstabilities ………….................................................................... 32

3.1 Interface Width in Breech of Nozzle ........................................................................................... 33

3.2 Linear Theory of Lower Hybrid Drift (LHD) Instability ......................................................................... 43

3.3 Quasi-Linear Evolution of LHD Instability and Anomalous Resistivity .............................................................46

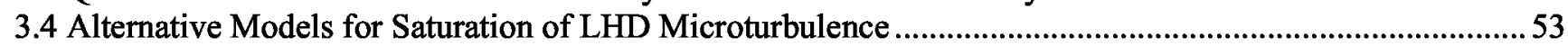

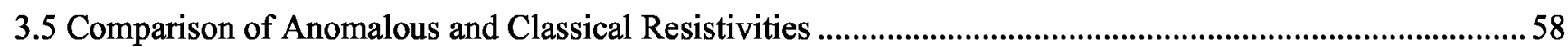

4.0 Rayleigh-Taylor- (RT-) Type Instabilities ........................................................................................................6

4.1 RT-Type Instabilities at Initial Impact With Magnetic Field ..................................................................................6 60

4.2 RT-Type Instabilities Downstream From Injection Region.............................................................................62

4.2.1 Surface Instability of Static Plasma in Region of Adverse Curvature .......................................................63

4.2.2 Surface Instability of Flowing Propellant in Region of Adverse Curvature ............................................66

4.3 Finite Larmor Radius (FLR) Stabilization of RT-Type Flute Modes ................................................................69

4.3.1 Example: Estimate of Hall Term in Coaxial MHD Thruster ………………………………………….......72

4.3.2 Concluding Thoughts ..................................................................................................................... 73

4.4 Short-Wavelength Gravity-Driven Instabilities.......................................................................................... 74

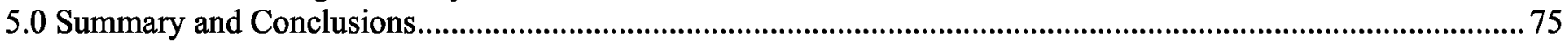

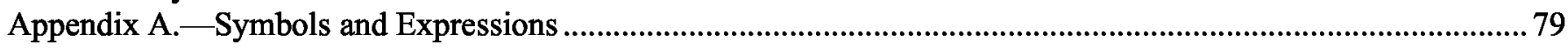

Appendix B. - Influence of Dwell Time on Plasma-Field Mixing Layer Width............................................................. 83

Appendix C. - Planar Similarity Solution for Resistive Diffusion of Field Into Plasma.............................................. 85

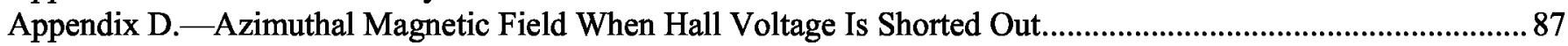

Appendix E. - Inward Radial Drift of Propellant ............................................................................................................ 89

Appendix F.-Derivation of Some Results Noted in Presentation of Initial Boundary-Layer Width......................... 91

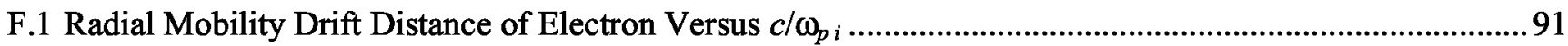

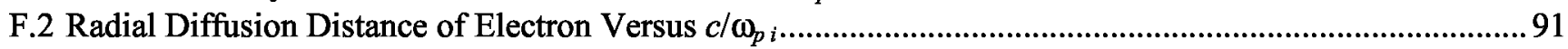

F.3 Fluid Deceleration Neglected in Ion Trajectory Equation ..............................................................................91

F.4 Azimuthal Current Carried by the Ions During One-Fourth of a Gyroperiod ………………….......................92

Appendix G. -Effect of Electron Pressure on the Initial Plasma-Field Mixing Layer Width.......................................93

Appendix H. - Relation Between Global Beta and Radial Deceleration ......................................................................97

Appendix I._- Initial Ratio of Ion Gyroradius to Plasma-Field Interface Width......................................................... 101 
Appendix J. - Generalized Plasma-Field Interface Thickness at the Throat Calculation and Example

J.1 Calculation of Plasma-Field Interface Thickness and Fraction of Attached Plasma Discussion and Example

J.2 Calculation of the Fraction of Attached Plasma-An Example

Appendix K.-Raleigh-Taylor Instability: Further Considerations

References 


\title{
Integrity of the Plasma Magnetic Nozzle
}

\author{
Richard A. Gerwin \\ Los Alamos National Laboratory \\ Los Alamos, New Mexico 87545
}

\section{Summary}

The injection of hot $(100 \mathrm{~s} \mathrm{of} \mathrm{eV})$ plasma propellant into a nozzle composed of shaped magnetic flux to convert the plasma thermal energy into directed thrust is fundamental to enabling high-specific-impulse (10 000s of seconds) and high-specific-power $(10 \mathrm{~s}$ of $\mathrm{kW} / \mathrm{kg})$ piloted interplanetary propulsion. This report pertains to the theoretical physics governing certain aspects of the flow of plasma propellant through a magnetic nozzle, primarily the integrity of the interface between the plasma and the nozzle's magnetic field, for these operational parameters. An expression for the initial thickness of the interface is derived and found to be significant (on the order of $10^{-2} \mathrm{~m}$ ). A comparison is made between classical resistivity and gradient-driven Lower Hybrid Drift microturbulent (anomalous) resistivity, from which an algorithm is derived that obtains interface thickening as a time integral, that is then related to the nozzle-shaped geometry of the interface. An algorithm characterizing the plasma temperature, density, and velocity dependencies is derived and found to be comparable to classical resistivity at local plasma temperatures on the order of $200 \mathrm{eV}$. Macroscopic flute-mode instabilities within the interface in regions of adverse magnetic curvature are discussed and a practical growth rate formula for magnetic nozzle design is derived. It is calculated that only one to two e-foldings of the most unstable Rayleigh-Taylor (RT) mode would occur. For a more complete treatment of the RT effect it will be necessary to include the Hall effect as well as ion magnetoviscosity. The necessity of incorporating the Hall effect into Ohm's law is discussed, where the full Hall current is able to flow and concomitant plasma rotation allowed. In that case, a critical nozzle length expression is derived below which the interface thickness is limited to about 1 ion gyroradius.

\subsection{Introduction}

This report pertains to the flow of plasma propellant through a nozzle composed of shaped magnetic flux, with application to the propulsion of space vehicles. The integrity of the interface between plasma and nozzle magnetic field is a particular concern. We consider at the outset a set of coaxial, circular, highly conducting coils in vacuum carrying azimuthal currents. Such a coil set produces a longitudinal magnetic field $\mathbf{B}$ having cylindrical vector components $B_{r}$ and $B_{Z}$. In terms of a "long-thin" geometric approximation, the latter component would be dominant. Plasma thrusters, in their simplest form, involve the nozzle-based acceleration of hot plasma propellant along the nozzle-shaped longitudinal magnetic field.

Figure 1 illustrates a simplified few-coil magnetic nozzle cross section without and with the propellant flowing, respectively. An engineering design would incorporate a large number of incremental contiguous coils. The magnetic nozzle configuration is nominally axially symmetric. It consists of a figure-of-revolution around the dash-dot axis of symmetry. The current-carrying magnetic-field coils are represented in cross section as rectangles. They carry adjustable azimuthal currents in the $\theta$ - (azimuthal angle) direction. The magnetic field lines produced by these coils lie in the meridional $r, Z$-plane. Some of them are represented here as solid lines closed around the coils (div $\mathbf{B}=0$ ). The chamber wall of the nozzle is not shown here, but would lie just inside of the magnetic field coils, at a slightly smaller radius.

Figure 1(a) illustrates magnetic field lines in vacuum, without injection of hot plasma propellant. The incipient nozzle breech, nozzle throat, and nozzle exit regions are indicated. An engineering design would incorporate a large number of incremental contiguous magnetic coils to smooth out and control the flow of plasma propellant.

Figure 1(b) illustrates magnetic field lines, before and after being distorted by conducting plasma propellant injected into the breech. Magnetic flux is squeezed between the highly conducting plasma propellant and the yet more highly conducting metallic magnetic field coils. In the idealized magnetohydrodynamics (MHD) model, the initial magnetic flux in the chamber volume never penetrates either the coils or the plasma. The dotted arrows indicate magnitude and direction of the plasma flow velocity. The highly conducting edge plasma flows along the distorted nozzle magnetic field lines. (The pressure of the conducting plasma distorts the initial magnetic field lines.) Concomitantly, the exterior magnetic field acts like a flexible containment wall, radially confining the interior plasma.

The directed plasma velocity is small in the breech and large at the exit. Stagnation enthalpy is converted into flow energy by the converging-diverging magnetic nozzle geometry. Magnetic trim coils may be needed beyond the exit region to straighten out the flow downstream.

Adjustments of details of the configuration geometry, as well as adjustments of the propellant's injection geometry, and the initial time dependence of the setting-up injection rate may be necessary to achieve a final desired configuration of steady flow. 

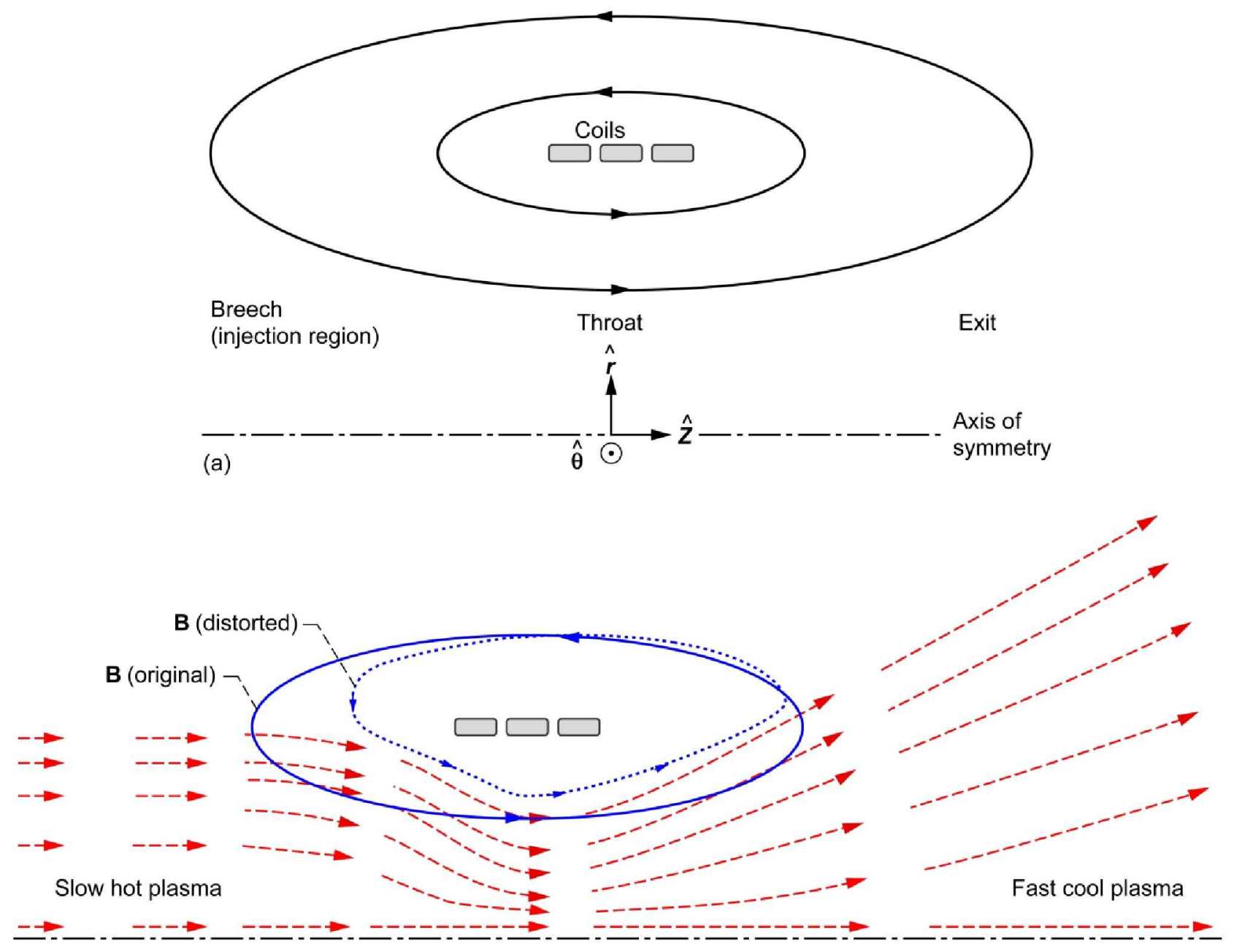

(b)

Figure 1.-Magnetic nozzle field. (a) In free space (no propellant). (b) Distorted by pressure of plasma propellant (length of plasma vectors denotes velocity magnitude).

Resistive diffusion of the squeezed magnetic flux into the slightly resistive external magnetic coils can be mitigated, in principle, by time-dependent programming of the coil's circuit currents or by the use of superconducting coils. Of course, neither of these options is available for the prevention of resistive interdiffusion of magnetic flux and plasma propellant. Certain instability mechanisms similarly cause mixing of plasma and magnetic field regions. These resistive and dynamical processes are both important because they degrade the performance of the magnetic nozzle, by allowing plasma attachment to the closed external magnetic field lines. The potential harm due to these processes thus constitutes the motivation for this report, which deals with the interface where the nozzle magnetic field meets the plasma.

When a highly conductive volume of plasma (centered on the axis) is axially driven into such a longitudinal magnetic field, azimuthal diamagnetic currents are induced to flow in the plasma. These induced plasma currents may be regarded as images of the external currents. The plasma currents are directed to reduce the internal magnetic field within the plasma and increase the field on the outside of the plasma. The net result is as if magnetic flux had been transferred from the plasma volume to the external volume. From Faraday's law, the total magnetic flux is conserved in the overall transverse cross section because the external coils are highly conducting. Pictorially, the intruding plasma pushes magnetic flux out of the way.

In an extreme idealization of this process (derived from very highly conducting plasma), all of the internal magnetic flux will have been expelled from the plasma to augment the external magnetic flux that confines the plasma laterally. Such confinement is affected by external magnetic pressure of the field (ultimately supported by the external coils). This external magnetic pressure acts inward across the plasma boundary to balance the outward lateral thermal and inertial pressure of the plasma.

The magnetic nozzle concept discussed in this report is based upon the above-described idealization (illustrated in 


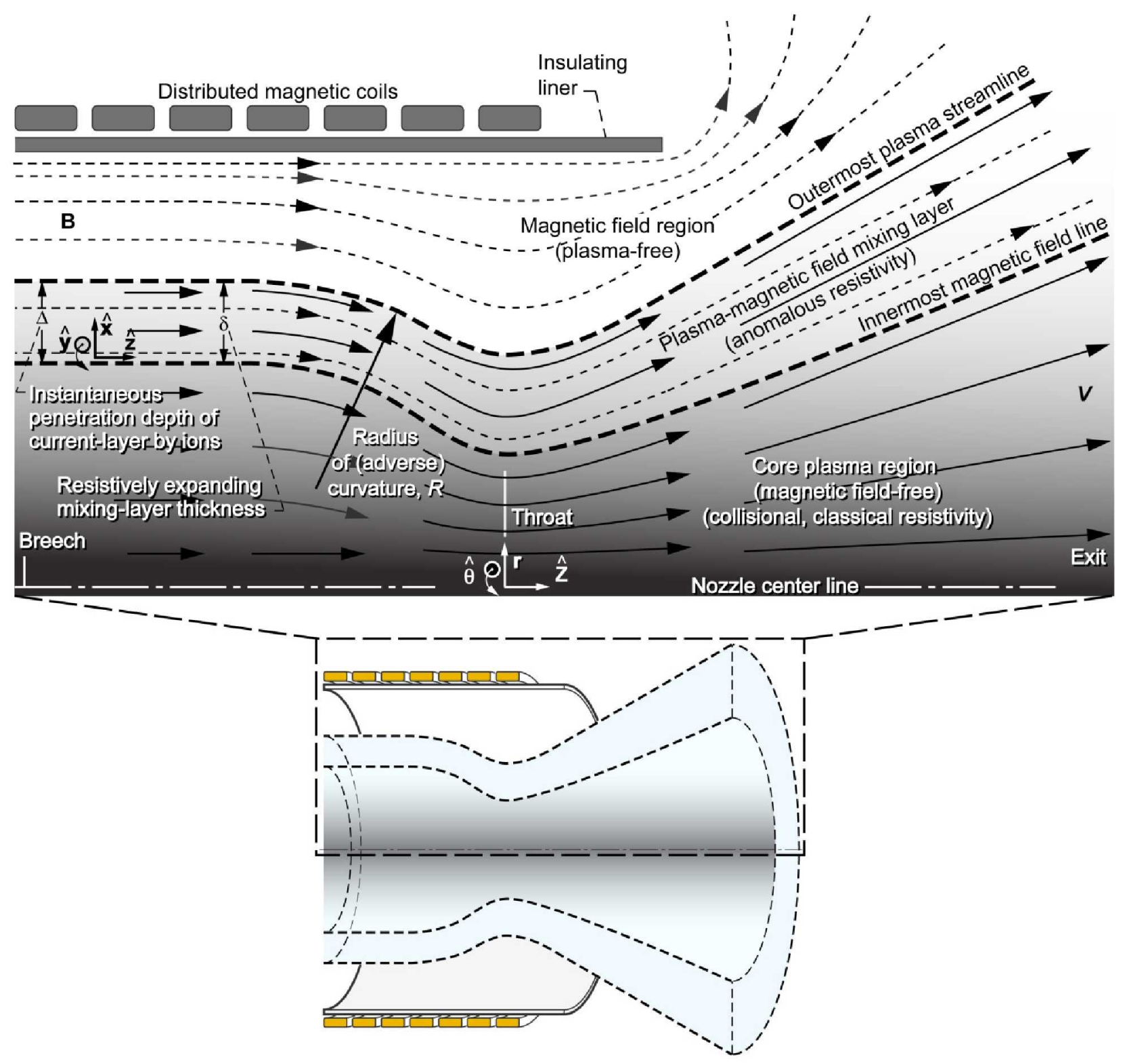

Figure 2.-Plasma magnetic nozzle (not to scale).

Fig. 1), notably incorporating a "sharp-boundary" model of the field-free plasma. Moreover, the chosen configuration is the best that could be hoped for from the viewpoint of the plasma-detachment problem, since no plasma resides on magnetic flux. The report focuses upon the degradation of this ideal zero-order configuration due to the occurrence of fundamental microphysical processes. Concomitantly, the detachment problem again becomes a concern.

A more realistic illustration that anticipates the degradation from an ideal configuration is shown in Figure 2. Starting at the top, distributed coils carry the electric current that generates the longitudinal magnetic field (B). An insulating liner is the physical surface that prevents the flow of radial current and thus provides the Hall voltage. (Without radial current, the edge plasma will not spinup.) The confining magnetic field occupies a region largely plasma-free near the coils and liner, where it is generally parallel to the axis of symmetry. The field then becomes more diffuse in the direction towards the axis of symmetry. This is the resistively expanding plasma-magnetic field (hereafter plasma-field) mixing layer $\delta$, possibly due to anomalous resistivity, which contains both plasma and magnetic field. The core plasma is envisioned to be field free.

Starting from the left in Figure 2, the initial penetration depth of ions into the confining magnetic field $\Delta$ is upstream of the throat. The resistively expanding plasma-field mixing layer $\delta$ increases from the initial penetration depth of the ions. A region of possibly Rayleigh-Taylor (RT) unstable plasma due to adverse longitudinal curvature may exist just upstream on the throat. A smooth throat is formed by gradually 
increasing the azimuthal current in the coils about halfway down the complete nozzle, then gradually decreasing the coil currents further downstream towards the exit. Beginning at the throat, there is a precipitous drop in plasma temperature, which continues downstream (so the plasma resistivity is likely to be classical downstream of the throat). Beyond the "Exit" there will be supplementary magnetic coils to straighten out the flow of the core plasma. This process will involve resistive energy losses.

The injection of hot hydrogen-plasma propellant into the breech of a magnetic nozzle, with subsequent conversion of its thermal energy into directed energy (and momentum), constitutes one of the important approaches to high-specificimpulse propulsion of space vehicles, as envisioned for certain interplanetary missions (Refs. 1 to 5). A magnetic field configuration comprising axially symmetric $\left(B_{r}, B_{0}\right)$ field lines (in terms of cylindrical coordinates), can be shaped into a converging-diverging nozzle by properly placed coaxial solenoidal coils. It offers advantages over wall-confined flow for the controlled nozzle flow application of a high-temperature fully ionized propellant gas.

One such advantage is derived from the nozzle magnetic field imposing an inward radial pressure balancing the outward plasma pressure, presumably isolating the plasma from the vessel wall (the coil shields). Such radial magnetic confinement of high-electrical-conductivity propellant can in principle reduce wall erosion by flowing plasma and reduce contamination of the plasma by heavy, high-atomic-number wall impurities. Radial magnetic confinement, if successful, thus mitigates unwanted mass entrainment and consequent reductions in specific impulse as well as mitigates the loss of thruster efficiency from inadvertent diversion of enthalpy into impurity radiation.

Another advantage is the opportunity for expeditious experimental development since propellant flow control along shaped magnetic field lines can be controlled and optimized in a convenient, iterative manner during operation. Such optimization can be realized by adjusting the size and placement of coils and their currents. Still another possible advantage may be magnetic control of the thrust vector if slight deviations from axial symmetry are tolerated near the nozzle exit.

Viability of the magnetic nozzle concept in its simplest form rests upon the integrity of a well-defined axially symmetric plasma-magnetic field interface. However, some fundamental microphysical processes, in the form of electron-ion collisions and microinstabilities, give cause for concern about the maintainability of a sharp interface. The sharp plasma edge adjacent to the surrounding magnetic flux constitutes an ideal axisymmetric equilibrium configuration in the sense of a pencil perfectly balanced on its point; it is nevertheless nonequilibrium in that there are closely accessible asymmetric configurations of lower energy. Such processes macroscopically broaden the interface by either classical or anomalous resistive diffusion, the former due to coulomb scattering of electrons on ions, and the latter due to time-dependent three-dimensional electric and magnetic fluctuations.

If the desired interface were to become diffuse because of the action of these processes, the consequent enhanced intermixing of the periphery of the propellant core with the inner edge of the magnetic nozzle field (propellant "attachment") would create a "detachment problem." Inadvertent attachment of propellant to magnetic flux becomes ultimately manifest as resistive drag acting on the attached portion of the exhaust plume. That is, the attached part of the exhaust plume axially stretches and bends the returning magnetic flux lines. Because axial stretching and bending of returning magnetic field lines requires energy, the energy drain is manifested as a resistive drag on the propellant's egress. Exhaust velocity and thrust thus become degraded. This situation motivates attention to the attachment process.

In this report, a process of "instant attachment" of newly injected propellant to the magnetic nozzle flux is described. It is not accessible to standard MHD simulations, being characterized by Hall effects and individual particle (kinetic) effects. Examination of interface physics during the first quarter ion gyroperiod after injection is performed. It shows an initial boundary-layer thickness in the breech of the nozzle, which proves to be on the order of the ion inertia length, $c / \omega_{p i}$ (the speed of light divided by the ion plasma frequency). This layer thickness in the breech of the nozzle constitutes an initial condition subject to further resistive broadening (Fig. 2).

One of the inferences of this report is as follows: Despite the substantial body of excellent theory and some limited experimental information that have been accrued, there is not yet a sufficiently comprehensive and accurate subgrid model readily available for magnetic nozzle design. The effects of nonzero beta (beta, $\beta$, is defined as the local ratio of total plasma pressure to magnetic pressure, $P_{\text {tot }} / P_{B}$, where $P_{B}=$ $B^{2} / 2 \mu_{0}$; thus, $\left.\beta=2 \mu_{0} P_{\text {tot }} / B^{2}\right)$ in gradient-driven microturbulence are not yet well understood, and $\beta$ ranges from practically zero in the external magnetic field to a large value within the internal plasma. Moreover, there is not yet agreement on the numerical factor in anomalous resistivity in the plasma edge layer, although there is agreement on the parameter dependencies. Such a model ideally would be applied in tandem with available two-dimensional axially symmetric MHD computer simulations (such as MACH2, see Ref. 3), with the intent to optimize the magnetic nozzle configuration while minimizing the detachment problem.

On the other hand, within the magnetic-fusion-energy community and within the space-plasma physics community, there exists the knowledge base and the expertise for development of the needed theoretical and computational tools. The development of such a synergistic design capability to supplement the MHD codes would require a highly detailed and computationally intensive effort by a team of scientists collectively familiar with both the numerical issues and the physics issues. 
The body of this report is intended to provide substance to these general remarks. Some of the key journal articles are reviewed without dwelling overly on their mathematical details. (It proved necessary to collect and study many papers to assimilate a certain perspective from which to identify key contributions.)

\subsection{Outline of Report}

Section 2.0, "General Physics Description of Magnetic Nozzle," presents general physics descriptions of magnetic nozzle flow, and the flow of a bounded, resistive fluid along magnetic nozzle field lines is discussed therein, along with associated processes that may be potentially deleterious to the plasma-field interface in the magnetic nozzle. The significance of local propellant resistivity and of local radial pressure gradients, in connection with diffusive broadening of the desired axially symmetric interface at the free-surface boundary of the propellant region, will be indicated. The eventual connection due to microturbulence between effective resistivity and the steepness of edge gradients will also be pointed out.

We emphasize in Section 2.0 that a local treatment of diffusive intermixing of plasma and field needs to be made well defined by specifying certain global electrophysical boundary conditions. The need arises because of the Hall effect, which introduces additional dominant physics considerations when the electron gyrofrequency in the plasma-field interface is much larger than the electron collision frequency. The global electrophysical boundary conditions directly influence the nature of the local Ohm's law, which, in turn, is a key player in calculating the evolution of the plasma-field interface.

The conventional application of the simple Ohm's law requires that the radial Hall voltage not be shorted out and concomitantly, that the Hall current not be allowed to flow. An important demonstration of this assumption lies in the opposite case, in which there ensues a severe reduction of effective plasma electrical conductivity due to Hall current, which is termed "magnetoresistance." Magnetoresistance would be a feature of any simulation or experiment that allows Hall current but disallows plasma rotation. A practical consequence of magnetoresistance germane to magnetic nozzle operation would be a severe enhancement in the rate of diffusive plasma-field intermixing. Such considerations would apply no matter whether the fundamental resistivity is classical or anomalous. In the magnetic nozzle geometry, however, having a flow of Hall current without rotation of propellant would be unrealistic. Therefore, rotation also has been included here, with attention to satisfying boundary conditions in the propellant injection region.

Allowing unhindered plasma rotation self-consistently with the presence of Hall current and with no Hall voltage drastically changes the character of the plasma-field transition layer from the artificial nonrotating case. We show that the layer thickness is limited to about the size of the ion gyroradius, provided that the nozzle length is shorter than a certain critical length. That length is $\lambda_{e} \sqrt{m_{i} / m_{e}}$, namely the product of a representative electron mean free path (possibly anomalously short) with the square root of the ion-toelectron mass ratio.

The quasi-radial Hall current produces an azimuthal $\left(\mathbf{J}_{\text {Hall }} \times \mathbf{B}\right)$ force that spins up the edge plasma. In a steadyflow model that satisfies the up-stream boundary conditions, the Hall-driven rotational velocity increases going downstream. The originally shorted Hall voltage is eventually reestablished downstream in the reference frame of the rotating edge plasma after sufficient spinup has been achieved. This explains why the interface layer broadening, downstream of the above-mentioned critical length, reverts to the resistive layer that it would have been had the Hall current not been allowed to flow. In this regard, it should be noted that viscous interaction of the plasma's edge layer with the core plasma would slow down the spatial rate of Hall spinup of the edge layer, and would therefore increase the above-mentioned critical length.

In Section 2.0 we suggest the possibility of backup approaches to magnetic nozzle utilization. In the event that a sharply defined plasma-field interface cannot be achieved and maintained throughout the nozzle, nozzle-based acceleration of attached plasma along with core plasma is to be expected because of the converging-diverging property of the attached external annular regions of magnetic flux. The problem of maximizing thrust then occurs at the nozzle exit, where accelerated attached plasma must be freed from returning magnetic flux. One approach to that detachment problem, which supports nozzle efficiency, has been documented, and the reference is listed.

Section 2.0 also contains a summary of nominal working parameters (density, temperature, and magnetic field strength) that may be relevant to some interplanetary missions and laboratory experiments. These are referred to occasionally throughout the report in connection with certain estimates of plasma properties.

In Section 3.0, "Resistivity From Gradient-Driven Microinstabilities," we first address the initial interface width (in the breech) of the plasma-field mixing layer. This result is relevant to an important microinstability, the Lower Hybrid Drift (LHD) instability, regarding its linear and nonlinear evolution. Then we review the linear theory for that microinstability, which is suspected to cause the broadening of magnetic, shear-free plasma-field interfaces. This is a relatively robust, gradient-driven small-scale instability. Some stabilizing features are pointed out.

Also within Section 3.0, the most difficult aspect of the microturbulence issue is addressed. It involves the connection of LHD microturbulence with anomalous resistivity, as arising from the nonlinear evolution and saturation of gradient-driven microinstabilities. The subject is reviewed and includes quasi-linear and alternative models. The review also discusses some attempts at numerical simulation. A 
practical formula for resistive interface broadening for general resistivity is identified, which can be applied to postprocessing of MHD simulations of magnetic nozzle flow. A comparison of classical and anomalous resistivity from one of the simulations is presented, as a function of plasma density and temperature in hydrogen propellant.

Section 4.0, "Rayleigh-Taylor- (RT-) Type Instabilities," deals with RT-type instabilities driven by adverse curvature. The presence of these RT modes leads to a lack of nozzlebased control of directed propellant flow. It is important to be aware that, in addition to diffusive broadening, the boundary surface of the propellant is free to deform, especially along the direction perpendicular to the field lines, namely the azimuthal direction. Deformations of the plasma surface in the azimuthal direction do not involve the expenditure of energy on local bending or stretching of field lines. The propellant's surface therefore is vulnerable to "flute modes," in spite of plasma pressure being nominally balanced by magnetic pressure at the interface. Specifically, regions of adverse streamline curvature and adverse magnetic curvature (center-of-curvature lies within the plasma) are susceptible to RT deformations (Fig. 2). The distinction between the propellant streamlines and the magnetic field lines is important in the injection region (see Sec. 4.0).

The RT modes will be discussed within the context of magnetic nozzle operation, including the effect of finite Larmor radius (FLR) stabilization. A physical derivation of the growth rate will be presented that incorporates the simultaneous influences of plasma pressure and plasma flow with curved streamlines. A practical formula for postprocessing use with axisymmetric MHD simulations of magnetic nozzle flow is obtained.

It is recognized that a more rigorous treatment of adverse curvature instabilities lies within the subject of "ballooning" modes, which takes into account that the adverse curvature regions have limited length. This is a complicated subject from which practical formulae applicable to magnetic nozzle flow are not readily available. It deserves a special treatment that is beyond the purview of this report. A reference to work on the destabilization of ballooning modes in mirror confined plasmas is given.

The FLR stabilization of the long-wavelength RT branch is reviewed and is utilized to construct a practical formula for nozzle design. Enhancement of the RT growth rate by Hall effects is mentioned, with references, but these are not included in this report. It is observed that adverse curvature of propellant streamlines in the injection region is practically unavoidable. The short-wavelength branch of the RT modes is also reviewed, including the absence of their FLR stabilization, as well as their close connection with the previously treated LHD instability. For the short-wavelength RT branch, the relative importance of curvature relative to the plasma pressure gradient is examined and found to be important in the plasma injection region.

Section 5.0 contains a final summary and discussion.
Appendix A provides a list of the symbols used in this report, and Appendixes $\mathrm{B}$ through $\mathrm{K}$ expand on various concepts presented in this report (see "Contents").

\subsection{Basic Notation and Basic Approach}

We use uppercase $V$ for macroscopic fluid velocities as in the MHD model and $V_{\text {th } i}$ for the ion thermal velocity. Lowercase $v$ is used for particle velocities, as in connection with the Boltzman-Vlasov equation.

The evolution of the diffusive plasma layer at the edge of the plasma is described with a local Cartesian coordinate system. This approach is valid whenever the thickness of the diffusive layer is small compared with the radius of curvature the short way around. As an example of the validity of this approach, see Section 2.3.3, "Diffusion of Field Into Plasma With Hall Effect," which describes the penetration of an external magnetic field into the plasma in full cylindrical coordinates. Whenever the diffusive magnetic layer is thin compared to the radius of curvature, then the radial variable $r$ varies negligibly over the region of interest. It thus cancels out as a constant, and the cylindrical diffusion equations reduce to a Cartesian representation of the diffusion process.

Appendix B suggests a general and simple computational approach for obtaining the evolution of the plasma diffusive edge layer. One uses the exact resistive diffusivity $D$ inside of the time integral. The time integral is replaced by an axial one-dimensional spatial integral involving the axial velocity. The axial velocity is expressed in terms of the density variation and the area variation along the axial direction using global mass flow conservation. The density, temperature, and velocity variations along the flow can be obtained from the quasi-one-dimensional model. If $D$ is based upon classical resistivity, one can take its temperature dependence from the quasi-one-dimensional model. If it is based upon anomalous resistivity, for example, from the LHD instability, one can also use the quasi-one-dimensional model for the other scaling variables.

\subsection{General Physics Description of Magnetic Nozzle}

In this section, we consider the physics associated with the momentum equations of the electrons and ions. The notation utilized is as follows. The magnetic nozzle field is composed of the vector field $\mathbf{B}=\left(B_{r}, 0, B_{Z}\right)$, referring to cylindrical coordinates $(r, \theta, Z)$. The magnitude of the vector $\mathbf{B}$ is $B$. The $Z$-axis constitutes the symmetry axis of the nozzle geometry. The unit vector along $\mathbf{B}$ is $\hat{\mathbf{b}}$. The plasma velocity vector (ion-fluid velocity vector) component along $\hat{\mathbf{b}}$ is $V_{/ /}$(see Fig. 2).

Let $\hat{\boldsymbol{\theta}}$ be a unit vector in the azimuthal direction. Then the velocity vector component across the flux surfaces in the 
quasi-radial direction $\hat{\mathbf{x}}=\hat{\boldsymbol{\theta}} \times \hat{\mathbf{b}}$ is $V_{\perp}$. The unit vector $\hat{\mathbf{x}}$ is in the direction normal to the axially symmetric flux surfaces (Fig. 2). Propellant rotation is presently precluded (but included later), so that the total velocity vector of the propellant now is $\mathbf{V}=V_{/ /} \hat{\mathbf{b}}+V_{\perp} \hat{\mathbf{x}}$.

The steady-state momentum equation for the plasma (suppressing viscosity) is obtained by adding the momentum equations of electrons and ions, while neglecting electron inertia and recognizing that electron-ion collisions cannot alter the total momentum:

$$
\rho \mathbf{V} \cdot \nabla \mathbf{V}+\nabla P_{\text {tot }}=\mathbf{J} \times \mathbf{B}
$$

Here, $\rho$ is the plasma mass density, $P_{\text {tot }}$ is the plasma pressure (electron pressure plus ion pressure), and $\mathbf{J}$ is the current density vector to be obtained from Ohm's law. When $V_{\perp}$ $\ll V_{l /}$, as is generally desired for magnetic-field guided flow of plasma, then the dominant components of Equation (1) along and across $\hat{\mathbf{b}}$ in the interface region are as follows:

$$
\begin{gathered}
\partial_{l}\left(\frac{V_{l l}^{2}}{2}\right)+\left(\frac{1}{\rho}\right) \partial_{l} P_{\mathrm{tot}}=0 \\
-\left(\frac{\rho V_{l /}^{2}}{R}\right) \hat{\mathbf{x}}+\nabla_{\perp} P_{\mathrm{tot}}=\mathbf{J} \times \mathbf{B}
\end{gathered}
$$

In Equation (2), $l$ is the distance along the local field line (or streamline in the field-free region of core plasma). In Equation (3), $R$ is the local longitudinal radius of curvature of the field line, and it is assumed for definiteness that the local curvature is adverse, that is, convex outwards. (If otherwise, replace $R$ by $-R$.) The symbol $\nabla_{\perp} P_{\text {tot }}$ represents the component of pressure gradient normal to the flux surfaces, along $\hat{\mathbf{x}}$, where $\nabla_{\perp}$ represents the component of the gradient vector along the quasi-radial direction.

It is assumed below that the plasma pressure is comparable to the magnetic pressure $(\beta \approx 1)$ in the plasma-field mixing layer; that is, for a nominal plasma-pressure versus magnetic-pressure confinement condition. This is a balance of pressures. Within the plasma core the local $\beta$ is therefore very large, and within the external magnetic field region the local $\beta$ is very small. The local $\beta$ is of order 1 within the mixing layer, but also globally the internal plasma pressure must balance the external magnetic pressure across the interface. The latter viewpoint corresponds to the concept of a "global beta."

Strictly speaking, Equation (2) as it stands really is only valid along the actual flow streamlines, which can differ from the magnetic field lines because of resistive diffusion. Because the flow vector in the plasma-field mixing layer is referred to the magnetic field lines, however, Equation (2), which leads to the Bernoulli equation, is only an approximation within the plasma-field mixing layer. Its validity depends upon $V_{\perp} / V_{/ /} \ll 1$. The flow streamlines of the plasma that have become attached to magnetic flux in the plasma-field mixing layer can be expected to differ only slightly from the magnetic field lines because of resistive diffusion.

In particular, one finds $V_{\perp} / V_{/ /} \approx 0.5\left(D / V_{/ /} \delta\right)$. Here, $D$ is a representative resistive diffusivity ( $D=\eta / \mu_{0}$ in mks units), and $\delta$ is the characteristic diffusive width of the plasma-field mixing layer. For the parameters of interest and assuming classical resistivity due to coulomb scattering, the ratio $V_{\perp} / V_{/ /}$proves to be on the order of 0.001 . Thus, the flow lines would approximately follow the field lines even if there were substantial anomalous enhancement of resistivity (in $D$ ). In this connection, it turns out that $\delta$ scales as $\sqrt{D}$, so an anomaly factor of 100 in $D$ only increases $V_{\perp} / V_{/ /}$by a factor of 10 .

\subsection{Bernoulli Equation for Nozzle Flow}

Use of the Bernoulli equation for nozzle flow illustrates the conversion of thermal energy into directed kinetic energythe fundamental operation of a convergent-divergent rocket nozzle. A simple example of this is provided when a person blows up a balloon and then lets the compressed gas stream out of the blow hole. The total momentum is zero if there are no external forces acting on the balloon; so when gas streams out the back, the balloon has to dart forward to preserve the total momentum at zero. The total energy is initially entirely thermal. The thermal energy has been converted into directed kinetic energy, so the gas temperature must drop. Temperature is a measure of thermal energy. If the gas were a plasma, classical resistivity would increase.

Integration of Equation (2), assuming that pressure is related to density adiabatically along a streamline, yields the Bernoulli equation. Thus, $P_{\text {tot }} \rho^{-\gamma}$ is constant along a streamline. By assuming that the adiabatic index $\gamma=1+2 / Q=5 / 3$ (where $Q$ is the degrees of freedom; i.e., 3 ), there are at least occasional collisions. The energy-balance equation in its entropy-production form yields this adiabatic relation when there are neither sources nor sinks of mass and heat along a streamline. The choice $\gamma=5 / 3$ assumes that charged particles undergo at least several collisions during their transit of the nozzle, so that each charged particle samples all three degrees of freedom.

The result of integrating Equation (2) along a streamline or flux line is

$$
\frac{V_{\| \prime}^{2}}{2}+\frac{S^{2}}{(\mathrm{\gamma}-1)}=\mathrm{constant}
$$


Here, $S$ is the local speed of sound, which is related to pressure and density by

$$
S^{2}=\frac{\mathrm{d} P_{\text {tot }}}{\mathrm{d} \rho}=\frac{Y P_{\text {tot }}}{\rho}
$$

Equation (4) can also be related directly to the equation of total energy balance. With help of Equation (5), Equation (4) becomes a statement of conservation of total energy per unit mass along the stationary flow configuration. That is, the sum of the kinetic energy per unit mass, the thermal energy per unit mass, and the work done by unit mass against the ambient pressure constitute a sum that is conserved along a streamline.

The Bernoulli equation displays the acceleration properties of a converging-diverging long-thin nozzle when one recalls the "choked flow" condition at the nozzle throat, namely that the flow velocity equals the sound velocity there. Denoting values in the throat by subscript " $t$,"

$$
V_{/ / t} \equiv V_{t}=S_{t}
$$

For simplicity, we shall assume infinite contraction and expansion ratios for the converging-diverging nozzle. It can be demonstrated, however, that a realistic moderate contraction ratio of 2, together with a moderate expansion ratio of 3 , yields results very close to those obtained from assuming these ratios to be infinite. A long-thin coaxial tube of fluid (either central or annular) of variable cross section $A$ has the property that mass flow rate $\dot{m}$ is conserved along the tube. Therefore, $V_{/ /} \approx 0$ is set in the breech (beginning) of the nozzle in Equation (4), because $A$ is assumed very large (infinite) in the breech. The "constant" in Equation (4) is then determined to be

$$
\text { constant }=\frac{S_{b}^{2}}{(\mathrm{Y}-1)}
$$

Here, $S_{b}$ is the speed of sound in the breech of the nozzle. Use of Equations (6) and (7) in Equation (4) yields the flowspeed of propellant in the throat,

$$
V_{t}^{2}=S_{t}^{2}=\frac{3}{4} S_{b}^{2}
$$

where $\gamma=5 / 3$ has been used.

The exit velocity or specific impulse can be obtained from Equation (4) by recalling that propellant cools down as it expands through the nozzle. For a fully expanded flow, Equations (4) with (7) then provide the exit velocity as follows:

$$
V_{e x}=\sqrt{3} S_{b}
$$

With Equation (8) this also implies that

$$
V_{e x}=2 V_{t}
$$

Use of the adiabatic relation in the sound speeds in Equation (8) then yields the ratio of ion density in the throat to ion density in the breech, namely as

$$
\frac{n_{t i}}{n_{b i}}=\left(\frac{3}{4}\right)^{3 / 2}=0.6495 \approx \frac{2}{3}
$$

Since temperature, $T$, scales adiabatically as $n^{2 / 3}$ along a streamline, Equation (10) implies that the ratio of temperature in the throat to temperature in the breech is given exactly by

$$
\frac{T}{T_{b}}=\frac{3}{4}
$$

Before applying the above results, a perspective regarding acceleration by nozzle flow is presented. The above principles of nozzle flow and the realization of high exhaust velocities govern not only the field-free core plasma, but also govern plasma that already has interdiffused with the field and become attached.

Three conditions, however, must be met for nozzle-based acceleration to be useful on attached plasma. First, electrical conductivity must be sufficiently large within the nozzle so that the plasma velocity is approximately confined along magnetic field lines, notwithstanding the presence of crossfield diffusion. Second, annular flux tubes carrying attached plasma must themselves exhibit convergence-divergence similar to the plasma core, so that nozzle-based acceleration occurs within each annular incremental flux tube. Third, the plasmadetachment problem must be addressed and solved at the exit.

Reference 6 and references therein discuss nozzle-based acceleration of highly conducting attached plasma inserted in the breech within coaxial magnetic flux tubes (no field-free plasma) and includes George Marklin's numerical calculation illustrating temperature reduction downstream of the throat of the nozzle. When longitudinal and azimuthal magnetic fields and longitudinal and rotating flows are all included, the nozzle-based acceleration process still can be reduced to a generalized Bernoulli equation. Reference 6 recognizes the importance of solving the detachment problem, but does not consider special shaping of diverging magnetic field lines for that purpose. This has been carried out in Reference 7, however, which does describe a means of successful detachment with minimal loss of efficiency, while assuming that downstream plasma has only classical resistivity.

Classical resistivity $\eta_{\mathrm{cl}}$ is the smallest possible. It therefore leads to the most difficult detachment problem. Because $\eta_{\mathrm{cl}}$ increases downstream as $T^{-3 / 2}$ due to cooling of the expanding flow, the detachment process with $\eta_{\mathrm{cl}}$ can be successfully effected (Ref. 7). It proves necessary to outfit the vehicle with coaxial solenoidal trim-coils. They must be placed downstream in the diverging region of the magnetic nozzle, in a manner that ensures that the magnetic nozzle field lines are only weakly divergent, thereby minimizing 
TABLE I.-SAMPLE PARAMETERS RELEVANT TO SPACE VEHICLE PROPULSION AND RELATED EXPERIMENTS

\begin{tabular}{|l|c|c|}
\hline \multirow{2}{*}{ Parameter } & \multicolumn{2}{c|}{ Propellant } \\
\cline { 2 - 3 } & Hydrogen & Helium \\
\hline Mass, $m$, AMU & 1 & 4 \\
\hline Number density (breech), $n_{b}, \mathrm{~cm}^{-3}$ & $1.0 \cdot 10^{15}$ & $1.0 \cdot 10^{15}$ \\
\hline Number density (throat), $n_{t}, \mathrm{~cm}^{-3}$ & $0.65 \cdot 10^{15}$ & $0.65 \cdot 10^{15}$ \\
\hline Temperature (breech), $T_{b}, \mathrm{eV}$ & 50 & 100 \\
\hline Temperature (throat), $T_{t}, \mathrm{eV}$ & 38 & 75 \\
\hline Magnetic field, ${ }^{\mathrm{a}} B, \mathrm{~T}$ & 0.20 & 0.35 \\
\hline Electron cyclotron frequency, $\omega_{c e} \mathrm{~s}^{-1}$ & $3.2 \cdot 10^{10}$ & $5.6 \cdot 10^{10}$ \\
\hline Electrical conductivity (throat), ${ }^{\mathrm{b}} \sigma_{t}, \mathrm{mho}^{\mathrm{m}} \mathrm{m}$ & $2.6 \cdot 10^{5}$ & $3.7 \cdot 10^{5}$ \\
\hline Resistive diffusivity (throat), $D_{t}, \mathrm{~m}^{2} / \mathrm{s}$ & 3.2 & 2.3 \\
\hline Electron collision frequency $\left(\right.$ throat), $\mathrm{v}_{e t}, \mathrm{~s}^{-1}$ & $0.8 \cdot 10^{8}$ & $1.0 \cdot 10^{8}$ \\
\hline Flow velocity (throat), $V_{t}, \mathrm{~m} / \mathrm{s}$ & $1.1 \cdot 10^{5}$ & $1.0 \cdot 10^{5}$ \\
\hline Exit velocity, ${ }^{\mathrm{c}} V_{\text {ex }}, \mathrm{m} / \mathrm{s}$ & $2.1 \cdot 10^{5}$ & $2.0 \cdot 10^{5}$ \\
\hline
\end{tabular}

${ }^{a}$ Local ratio of thermal to magnetic pressure $\beta=1$ in breech.

${ }^{b} \ln \Lambda=10$, where $\Lambda$ is length of breech to throat divided by ion gyroradius.

${ }^{c}$ Also, specific impulse $\left(10^{-1} \mathrm{~s}\right)$.

resistive-drag losses. The addition of such trim coils then becomes a factor in mission considerations dealing with size and weight of the vehicle. It may be that the occurrence of enhanced resistivity in the downstream region would further ease the detachment problem.

The above Bernoulli results on nozzle flow are now applied to hydrogen propellant, with parameters similar to those relevant to interplanetary travel. Also, the Bernoulli results are applied to helium propellant with parameters similar to those proposed by Turchi (Ref. 2) for a magnetic nozzle experiment at Ohio State University using the 1-MJ, 1-GW pulsed-power source. Table I presents approximate round-number values of nominal reference parameters assumed to be inserted into the breech of the nozzle. The quantities are derived according to the Bernoulli equation for the throat and exit regions of the nozzle.

It is noted that the assumed high-enthalpy plasmas provide a desired specific impulse of about $20000 \mathrm{~s}$. Also, only moderate magnetic field strength is required to effect an initial balance between plasma pressure and magnetic pressure. (Downstream of the breech, one expects the magnetic flux to press inward in radius as the plasma's internal pressure becomes converted into longitudinal flow energy.) Hydrogen propellant is assumed to be axially inserted into the breech of the nozzle and preheated to the desired temperature by drawing off edge plasma into the nozzle from the fusion power reactor.

From Table I, a feature that proves to be very important with regard to the Hall effect is observed. The electron cyclotron frequency in the edge plasma $\omega_{c} e$ largely exceeds the electron coulomb collision frequency, $v_{e}$, by more than 2 orders of magnitude. In the throat, magnetic field $B$ is still
0.7 of its breech value, hence, also $\omega_{c} e^{\text {Even a severely }}$ anomalous electron collision frequency still could be dominated by the electron cyclotron frequency.

At a fixed temperature in the breech, the classical collision frequency there is proportional to $n / T^{3 / 2}$ whereas the electron cyclotron frequency there is proportional to $\sqrt{n T}$ via pressure balance. Thus, for the given temperature, the reference density would have to be increased by orders of magnitude and out of range in order to bring the electron's coulomb collision frequency into the neighborhood of the electron cyclotron frequency. For these reasons, the Hall effect in the mixing layer is regarded as sufficiently important to merit a separate treatment below.

Finally, we note that the velocity in the throat region, $V_{t}$, together with the envisioned meter-scale length of the nozzle, can be used to provide a rough estimate of the transit-time duration available for diffusive spreading of the plasma-field mixing layer. For a meter-length nozzle, this longitudinal transit time is on the order of $10 \mu \mathrm{s}$. (In Appen$\operatorname{dix} B$ it is shown that if we allow for the transit time to increase from zero velocity in the breech, there is an amplification of the transit time by a factor of $\sim 2$.)

\subsection{Diffusive Intermixing in Plasma-Field Interface}

This subsection addresses diffusive intermixing of plasma into magnetic field and also resistive diffusion of magnetic field into plasma. Both mixing processes occur together. We turn now to Equation (3) for crossfield diffusion of plasma.

\subsubsection{Diffusion of Wall-Confined Plasma}

Equation (3) is discussed as an illustration for the worst case. This case is one where hot plasma, initially inserted into the breech of the nozzle, inadvertently immediately spreads over all upstream flux surfaces (plasma-wall interactions are ignored). The plasma profile is assumed to extend uniformly from the symmetry axis out to the wall, so that the transverse pressure gradient is now neglected relative to the centrifugal force. Effective gravity from centrifugal force with adverse curvature would cause wall-confined plasma to sink against the outer wall, creating a pressure gradient that is positive outwards. By neglecting this pressure gradient, we will overestimate the crossfield velocity of plasma and the concomitant resistive deviation of the streamline from the field line. But even with uniform pressure, the plasma is still wall confined. Then Equation (3) reads

$$
-\left(\frac{\rho V_{\| /}^{2}}{R}\right) \hat{\mathbf{x}}=\mathbf{J} \times \mathbf{B}
$$

At the same time, assume that Ohm's law holds in its simplest resistive form, namely 


$$
\sigma \mathbf{V} \times \mathbf{B}=\mathbf{J}
$$

where $\sigma$ is the electrical conductivity of plasma $(\sigma=1 / \eta)$ and $\mathbf{V} \times \mathbf{B}$ is the effective electric field in the moving reference frame of the plasma, driving the current density.

Equation (13) implies that

$$
\mathbf{J} \times \mathbf{B}=-\sigma B^{2} \mathbf{v}_{\perp}
$$

It should be observed that this MHD force density, as obtained from the simple Ohm's law, is directed oppositely to the crossfield flow velocity. This phenomenon is known as "eddy current braking" of the plasma crossfield flow. The same phenomenon creates the detachment problem at the nozzle's exit.

Use of Equation (14) in Equation (12) presents a steadyflow balance between the outward centrifugal force resulting from parallel flow along a curved field line and the MHD braking force. As a concrete example, we shall evaluate this balance just upstream of the throat, where the field lines might be curving radially inwards towards the throat with an adverse curvature (of course, it also is possible to imagine a nozzle field with good curvature everywhere). This force balance can be expressed as a ratio of transverse to longitudinal velocities in the following form:

$$
\frac{V_{\perp}}{V_{/ /}}=\left(\frac{W_{\mathrm{kin}}}{W_{\mathrm{mag}}}\right)\left(\frac{D}{R V_{/ /}}\right)
$$

Here, $W_{\text {kin }}=\rho V^{2} / 2$ is the kinetic energy density of parallel flow approaching the throat region; and $W_{\text {mag }}=B^{2} / 2 \mu_{0}$ is the magnetic energy density near that region. Also, $D_{c l}=\left(\sigma_{c l} \mu_{0}\right)^{-1}$ is the classical resistive diffusivity there (Russian or Soviet papers sometimes refer to $D$ as "magnetic viscosity"). The magnetic field is chosen to have $\beta$ of order unity, and the flow speed is on the order of the speed of sound. (Even though $\beta$ is of order unity, it is assumed that the wall takes up the plasma pressure in the present example.) Therefore, the first nondimensional ratio, the ratio of energy densities in Equation (15), is of order unity. The principal determining factor for the velocity ratio $V_{\perp} / V_{/ /}$is the second nondimensional ratio in Equation (15). For $R=1 \mathrm{~m}$, the hydrogen parameters yield $D_{c l} / R V_{/ /} \approx 3 \cdot 10^{-5}$, and the helium parameters yield $D_{c l} / R V_{/ /} \approx 2 \cdot 10^{-5}$.

These very small numbers for $D_{c l} / R V_{/ /}$signify that flowing plasma having a diffuse profile remains very well attached to field lines $\left(V_{\perp} \ll V_{/}\right)$, assuming classical resistivity. This situation would still hold true even for $R=0.1 \mathrm{~m}$. It can therefore be expected that with the simple Ohm's law with classical resistivity and for the area of each incremental annular flux tube having a converging-diverging dependence on axial distance, nozzle-based acceleration still can occur for diffuse attached plasma. Moreover, it is worth noting that the small ratio $V_{\perp} / V_{/ /}$is so small indeed that the plasma resistivity could even be anomalously large by several orders of magnitude and yet have the plasma remain fairly well guided by the field lines.

To summarize Section 2.2.1, it can be stated that diffuse wall-confined plasma preattached to the magnetic flux can usefully acquire nozzle-based acceleration provided that the consequent detachment problem is solved. Reference 7 has described an approach to solving the detachment problem, which involves implementation of a weakly diverging magnetic nozzle field in the exhaust region.

\subsubsection{Diffusion of Magnetically Confined Plasma}

We return now to Equation (3), assuming that the edge plasma at the interface has such a sharp boundary that the transverse pressure gradient (negative outwards) completely dominates the centrifugal force term. Use of the simple Ohm's law, Equations (13) and (14), then yields an equation for the transverse velocity in terms of the transverse pressure gradient, $\nabla_{\perp} P_{\text {tot }} \approx-\left(P_{\text {tot }} / \delta\right) \hat{\mathbf{x}}$ :

$$
\mathbf{V}_{\perp}=-\left(\frac{\eta}{B^{2}}\right) \nabla_{\perp} P_{\text {tot }} \approx \frac{D \beta}{2 \delta} \hat{\mathbf{x}}
$$

Here, $\eta$ is the resistivity, $\eta=1 / \sigma$, and $D$ is again resistive diffusivity $D=\eta / \mu_{0}$. Also, $\beta$ is the ratio of thermal to magnetic pressure within the interface; $\beta=2 \mu_{0} P_{\text {tot }} / B^{2}$, where $P_{\text {tot }}$ is the sum of electron and ion pressures, $P_{\text {tot }}=P_{e}+P_{i}$. Finally, $\delta$ represents the thickness of the transition layer of magnetically confined plasma. The pressure gradient of confined plasma is noted to be inward, along $-\hat{\mathbf{x}}$, where $\left|\mathbf{V}_{\perp}\right|=V_{x}$.

From Equation (16), one then obtains the estimate

$$
\frac{V_{\perp}}{V_{/ /}} \approx \frac{\beta}{2}\left(\frac{D}{V_{/ /} \delta}\right)
$$

Equation (17) for the magnetically confined plasma has the same structure as Equation (15) for the wall-confined plasma, but now with a very small plasma-field mixing layer width in the denominator. However, even if $\delta$ were 4 orders of magnitude smaller than $R$, for example $\delta=0.01 \mathrm{~cm}$, one would still have a relatively small transverse velocity, namely $V_{\perp} \approx 0.1 V_{/ /}$. This can be seen by utilizing the same parameters as for Section 2.2.1. Moreover, if $\delta \approx 1 \mathrm{~cm}$, comparable to an ion gyroradius, then $V_{\perp} \approx 0.001 V_{/ /}$. Thus, 
qualitatively it appears that classical resistivity, with the given parameters, does not destroy the small ratio of transverse to longitudinal velocity, even for rather thin plasmafield mixing layers.

The analysis leading to Equation (17) can be extended. We take into account that the plasma-field mixing layer width $\delta$ is related to the transverse diffusion velocity $V_{\perp}$. The two really should be treated together in a self-consistent manner. To exploit this point of view, we cancel $V_{/ /}$from each side of Equation (17), and make the reasonable identification within the plasma-field mixing layer:

$$
V_{\perp}=\frac{\mathrm{d} \delta}{\mathrm{d} t}
$$

This equation represents the rate at which the plasma-field mixing layer thickens with time as the flow is followed downstream. Use of Equation (18) in Equation (17) and multiplying through by $\delta$ then yields

$$
\frac{\mathrm{d}\left(\delta^{2} / 2\right)}{\mathrm{d} t} \approx \frac{\beta D}{2}
$$

Assuming that the initial boundary-layer width of attached plasma in the breech is negligibly small (corrected in Sec. 3.0, "Resistivity From Gradient-Driven Microinstabilities,"), this differential equation suggests the estimate

$$
\delta \approx \sqrt{\beta D t_{b t}}
$$

where $t_{b t}$ is the time for longitudinal flow to traverse the nozzle, say from breech to throat. (The integration of Eq. (19) over time is addressed in Appendix B.) For a propellant whose transverse pressure is balanced by confining magnetic pressure, the quantity $\beta$ will be of order unity within the plasma-field mixing layer. A more rigorous and detailed analytic procedure that includes a non-MHDderived initial layer thickness is considered beyond the scope of this report (see Sec. 3.0, "Resistivity From GradientDriven Microinstabilities").

Note that Equation (20) assumes that there is no significant initial thickness of the plasma-field transition layer in the breech of the nozzle. It is appropriate for comparison to MHD simulations that make the same assumption. This issue, which cannot be fully addressed within the resistive MHD model, is considered later in this report in Section 3.1, "Interface Width in Breech of Nozzle." It is assumed here that the field coils and the injection process are such as to allow a macroscopically gentle injection, such that there are no anomalous or extreme dynamical macroscopic processes that produce an initial width of the transition layer in the breech of the nozzle. Otherwise, such a macroscopic initial layer thickness certainly would have to be included in the contribution to the layer thickness downstream. See Reference 1.

The terse derivation of Equation (20) will now be supplemented with a more rigorous derivation, which provides a more precise interpretation of the symbol $\beta$. It turns out that $\beta$ is neither a local nor a global concept here, but has aspects of both.

For slow, noninertial resistive flow in the interface, one has a situation of quasi-equilibrium.

$$
\nabla P=\mathbf{J} \times \mathbf{B}
$$

The quasi-radial component of this equation, outwards across the flux surfaces, involves only the azimuthal component of the current density. However, the azimuthal electric field vanishes in an axially symmetric steady state, so that the azimuthal current density can be represented in Ohm's law by

$$
\eta \mathbf{J}=\mathbf{V} \times \mathbf{B}
$$

which involves the outward cross-flux velocity of plasma. Use of the latter equation in the former yields

$$
D \nabla P=-2\left(\frac{B^{2}}{2 \mu_{0}}\right) \mathbf{V}
$$

in which the gradient and the velocity are directed outwards across the flux surfaces, in the quasi-radial x-direction. Here, $D$ is the resistive diffusivity $\eta / \mu_{0}$.

In the absence of a detailed model of the interface, one must have recourse to reasonable but expeditious assumptions to go forward with the theory. In this instance, such an assumption is that the plasma outward velocity corresponds to the rate of thickening of the interface width $\delta$.

$$
V_{x}=\frac{\mathrm{d} \delta}{\mathrm{d} t}
$$

Integrating the quasi-equilibrium equation from $x=0$ at the core edge to $x=\delta$ at the vacuum end of the interface, we then have

$$
-D P_{\text {core }}=-2\left\langle\frac{B^{2}}{2 \mu_{0}}\right\rangle \delta \frac{\mathrm{d} \delta}{\mathrm{d} t}
$$

In the above equation, $P_{\text {core }}$ represents the plasma pressure at the edge of the core region, and the brackets represent an average of the enclosed quantity over the interface width, $0 \leq x \leq \delta$. This equation also can be written as 


$$
\frac{\mathrm{d} \delta^{2}}{\mathrm{~d} t}=D \beta
$$

in which $\beta$ is now given by

$$
\beta=\frac{2 \mu_{0} P_{\text {core }}}{\left\langle B^{2}\right\rangle}
$$

In subsequent equations that represent resistive thickening of the interface by resistive diffusion, $\beta$ should be accorded this interpretation.

For the transit time $t$ we invoke $t_{b t}=L_{b t} / V_{t}$, where $L_{b t}$ is regarded as a characteristic length of the nozzle from breech to throat (similarly, $L_{b}$ ex is the characteristic length of the nozzle from breech to exit plane). Also, $V_{t}$ represents velocity in the throat region, which is known to be on the order of the ion thermal velocity. Then, from Equation (20), it can be shown that the square of the boundary-layer width is

$$
\delta^{2} \approx 2 a_{i}^{2}\left(\frac{L_{b t}}{\lambda_{e}}\right) \sqrt{\frac{m_{e}}{m_{i}}}=\beta\left(\frac{c}{\omega_{p i}}\right)^{2}\left(\frac{L_{b t}}{\lambda_{e}}\right) \sqrt{\frac{m_{e}}{m_{i}}}
$$

Here, we have retained $\beta$ in Equation (20) and have set the electron and ion temperatures equal, $T_{e}=T_{i}$. Retention of beta in Equation (28) is practically just a formality. The square root of the local beta is near 1 within the interface and so is the square root of the "combination beta" described above. The model of the interface invoked here is just not sufficiently detailed as to provide more accuracy than is available with such numerical factors. The ion thermal gyroradius is connected to the ion inertia length by the square root of the local beta. So, the factor that is being suppressed in delta, as given by the second part of Equation (28), is the square root of the ratio of the combination beta to the local beta. The square root of that ratio is expected to be near 1 . Also, the resistivity $\eta$ in $D$ in Equation (20) has been reexpressed in terms of its fundamental factors for hydrogen plasma; $\eta=m_{e} v_{e} / n_{e} q^{2}$, where $m_{e}$ is electron mass, $n_{e}$ is electron number density, $q$ is the magnitude of the electron charge, and $v_{e}$ is the momentum-transfer collision frequency of an average electron with background ions or with microturbulent fluctuating fields. The negative charge of an electron will be explicitly indicated as $-q$; thus $q$ is a positive quantity.

Recall that the resistive diffusivity $D=\eta / \mu_{0}$ in mks units, and $D=c^{2} \eta / 4 \pi$ in cgs units. Here, $\mu_{0}$ is the magnetic permeability of vacuum, and $c$ is the speed of light in vacuum. It is useful to note that $c^{2}=1 / \varepsilon_{0} \mu_{0}$ in mks units, where $\varepsilon_{0}$ is the electrical permittivity of vacuum.

In Equation (28), $a_{i}$ is a representative ion gyroradius in the attached plasma within the plasma-field mixing layer, $a_{i}^{2}=\left[2 T_{i} / m_{i} \omega_{c i}^{2}\right]$, with $\omega_{c i}$ being the ion gyrofrequency in the boundary-layer magnetic field. Moreover, $\lambda_{e}$ is a representative mean free path for collisions of electrons (possibly anomalous) with ions; hence $\lambda_{e}=v_{e}^{-1} \sqrt{2 T_{e} / m_{e}}$, and $m_{e} / m_{i}$ is the electron-to-ion mass ratio. Furthermore, $\omega_{p i}$ is the ion plasma frequency, with $\omega_{p i}=\sqrt{4 \pi n q_{i}^{2} / m_{i}}$ in cgs units and $\sqrt{n q_{i}^{2} / \varepsilon_{0} m_{i}}$ in mks units. The ratio $c / \omega_{p i}$ is called the ion inertia length.

The ion gyroradius $a_{i}$ comprises a principal feature of the boundary-layer width. Other highly significant factors also codetermine the resistive MHD boundary-layer width in Equation (28). Thus, $\delta / a_{i}$ depends upon the dimensionless longitudinal distance traveled, in the form $\left(L / \lambda_{e}\right)^{1 / 2}\left(m_{e} / m_{i}\right)^{1 / 4}$.

Suppose $L=1 \mathrm{~m}$. The throat velocity is $V_{t}=10^{5} \mathrm{~m} / \mathrm{s}$; hence the transit time is about $t=10^{-5} \mathrm{~s}$. Assuming $\beta \approx 1$, and taking classical resistive diffusivity $D_{\mathrm{cl}} \approx 3 \mathrm{~m}^{2} / \mathrm{s}$ for hydrogen, one finds from Equation (20) that $\delta \approx 0.6 \cdot 10^{-2} \mathrm{~m}=0.6 \mathrm{~cm}$, with a slightly smaller value for helium propellant. This value of $\delta$ constitutes an estimate for the width attained by the plasma-field mixing layer after following propellant up to and just through the nozzle throat. It is generated by resistive diffusion of plasma into the magnetic nozzle field. It overestimates the diffusion, since $D_{\mathrm{cl}}$ is the throat value with temperature reduced from its value in the breech. It underestimates the layer thickness, however, by neglecting any initial thickness. This neglect is appropriate for comparison of layer thickness $\delta$ with that from resistive MHD simulations.

In the assumed case of classical resistivity, with $\beta$ of the order one and taking parameter values listed in Table I above, broadening of the plasma-field mixing layer after passage of propellant up to and just through the nozzle throat thus proves to be rather small compared to system dimensions. This would be so even for a modest plasma throat radius of $10 \mathrm{~cm}$. Any simulation (or experiment) of magnetic nozzle flow of nominally confined plasma having those parameters and giving results with substantially larger diffusion of the plasma-field mixing layer may be violating one or more of the model assumptions set forth above. Also, the simulation may contain some numerical diffusion.

In this simple presentation of radial resistive diffusion, we have used a constant characteristic nozzle flow velocity for estimating $\delta$, which has been taken to be the velocity in the throat region of the nozzle. This procedure serves to define a characteristic dwell time as a characteristic axial length 
divided by the characteristic longitudinal velocity. However, the dwell time of the propellant between breech and throat actually is somewhat longer due to the time needed for the propellant to accelerate up to its characteristic velocity. This increased dwell time then gives the resistive plasma-field mixing layer more time to grow. The effect of the more accurate dwell time is discussed in detail later, in Section 2.4, "Summary," and in Appendix B. It is estimated that the time needed for acceleration engenders a factor of $\sim 2$ in the increased thickness of the plasma-field mixing layer.

\subsubsection{Diffusion of Confining Magnetic Field}

In the preceding case, diffusion of plasma into the confining field was considered. There also is another process for attaching plasma to the confining field. After the ambient magnetic field has been pressed against the outer conducting wall by the initial pressure of injected hot plasma, the field can rebound spatially downstream, by undergoing inward diffusion into the core plasma regarded as a resistive medium. Turchi analytically treated this view of plasma attachment (diffusion of a pulsed magnetic field into a resistive medium), using a well-known model of time-dependent magnetic diffusion (Ref. 8, personal communication), based upon a discussion of time-dependent magnetic diffusion. We now analyze this magnetic diffusion process by a slightly different method than that used by Turchi. The treatment here of the diffusion of a magnetic field into a conductor agrees (to within a trivial numerical factor) with that presented in Section 2.2.2 of plasma diffusion across a magnetic field. The reason is that the square root of beta is near 1 for the special beta described.

The point of view adopted here is that the exact time dependence of the magnetic field experienced at the boundary of the moving plasma is regarded to be less important than the overall time scale for that time variation. The reason is that the extent of the field diffusion into the plasma is obtained by time integration. This point of view thus motivates us to choose a simple but expeditious time dependence, containing a certain relevant characteristic time, for the magnetic field experienced by the moving plasma at its boundary.

In a reference frame moving downstream with the plasma velocity comparable to the velocity in the throat $\sim V_{t}$, where that velocity is assumed constant and uniform, Maxwell's equations are written as follows. Here, $\mathbf{E}^{\prime}=\mathbf{E}+\mathbf{V} \times \mathbf{B}$ is the azimuthal electric field in the moving frame.

$$
\begin{aligned}
\nabla \times \mathbf{E}^{\prime} & =\nabla \times(\eta \mathbf{J})=\nabla \times(D \nabla \times \mathbf{B}) \\
& =-D \nabla^{2} \mathbf{B}=-\partial_{t} \mathbf{B}
\end{aligned}
$$

In an axially symmetric configuration, one can note the following property of this set of equations. The longitudinal component (along B) involves the spatial variation - in the direction normal to the flux surfaces - of azimuthal vector components of electric field $\mathbf{E}^{\prime}$ and current density $\mathbf{J}$.

Here, it is important to note two other key features to be used in Equation (29). First, in the moving frame, the azimuthal electric field $\mathbf{E}^{\prime}$ will not necessarily vanish, even in a situation of steady flow and steady fields in the lab frame. Second, the azimuthal current density vector $\mathbf{J}$ is assumed related to the azimuthal electric field $\mathbf{E}^{\prime}$ (in the moving frame) by the simple Ohm's law, as before. Since $\mathbf{J}$ involves the difference between two vector velocities, those of electrons and ions, its evaluation in any frame produces the same result for quasi-neutral nonrelativistic plasma. Also, the magnetic field is the same in the moving frame, when the velocity is nonrelativistic. The resistive diffusivity $D$ in Equation (29) is assumed here to be a scalar constant representative of the throat region of the nozzle.

Cylindrical geometry can be suppressed when examining magnetic diffusion in the vicinity of the plasma's sharp boundary, as long as the plasma-field mixing layer width is small compared to its radial distance from the axis of symmetry. Accordingly, if we let the $x$-coordinate represent the direction normal to the flux surfaces (so that the distance $x$ increases towards the wall, see Fig. 2), then the last part of Equation (29) reduces to a simple diffusion equation within the plasma, considered as a resistive medium:

$$
\partial_{x}^{2} \mathbf{B}=\frac{\partial_{t} \mathbf{B}}{D}
$$

There is no loss of generality in setting $x=0$ at the plasma edge. This situation is effectively the same as a magnetic field diffusing into a conducting half-space bounded by a planar surface.

Following the line of Turchi's argument in a general way, we shall represent the time variation of the magnetic field experienced by the longitudinally moving plasma with simple exponential time dependence. The method is a form of the "separation-of-variables" technique for solving partial differential equations. Thus,

$$
B(x, t)=G(x) e^{\left(t / t_{b t}\right)}
$$

The incoming conducting plasma in the region upstream of the throat presses the external field against the metallic wall (coils), thus increasing the field strength in the local neighborhood. By assuming this monotonic increase with time of the magnetic field strength seen by the moving edge plasma during its traverse up to the throat region, the penetration of field into plasma shall surely be overestimated. (Using the plasma temperature in the throat, one overestimates the prethroat classical resistive diffusivity $D$.) Then, using Equation (31), Equation (30) is reduced to 


$$
G^{\prime \prime}(x)=\frac{1}{D t_{b t}} G(x)
$$

Here, $G^{\prime}(x)=\mathrm{d} G / d x$. The solution of Equation (32) within the plasma region $(x<0)$ is

$$
G(x)=C_{\mathrm{I}} e^{-|x| \sqrt{1 / D t_{b t}}}
$$

where $x$ is the positive (decreasing plasma density) or negative (increasing plasma density) distance from the plasma boundary (Fig. 2). This spatial profile within the plasma, engendered by the time-dependent magnetic field imposed at the plasma boundary, clearly exhibits the character of a boundary layer of magnetic field protruding into the interior of the plasma.

The moving plasma would generally experience significant changes in field strength of a time on the order of the plasma's transit time up to the nozzle throat. Therefore, it is reasonable to regard the time constant for imposed field growth as $t_{b t}$, which is the plasma's transit time through the converging part of the nozzle. Then, the plasma-field mixing layer thickness implied by Equation (33) can be expressed as

$$
\delta=\sqrt{D t_{b t}}
$$

where $t_{b t}$ is the nominal transit time through the converging part of the nozzle, $t_{b t} \approx L_{b t} / V_{t}$. Thus, essentially the same answer is reached for field diffusion into plasma as in Subsection 2.2.2 (assuming $\beta$ is of order 1) when the plasma diffuses into the field. In both cases, for the given parameters and classical resistivity, the plasma can be expected to intermix with the field to a distance of at most about $1 \mathrm{~cm}$ during transit through the converging part of the nozzle. This distance is to be compared to a system length on the order of $1 \mathrm{~m}$ and a plasma throat radius perhaps on the order of $10 \mathrm{~cm}$.

For comparison, the steady-state magnetic-field diffusion problem is solved in the lab frame in Section 2.3.3, "Diffusion of Field Into Plasma With Hall Effect," and in Appendix C. In the limit of simple resistive diffusion, a similarity solution is found from which a characteristic penetration depth evolves with increasing axial distance downstream. That result proves to be in very good agreement with the present treatment in the moving frame.

\subsection{Hall Effect, Plasma Rotation, and Electron Pressure in Ohm's Law}

We consider the foundations of the simple form of Ohm's law within the context of magnetic nozzle physics. Certain global conditions prove to be required in order for the simple Ohm's law to provide a valid description. This circumstance may influence the nature of the computed plasma-field mixing layer and its spatial rate of broadening.

The momentum equation of the electron fluid constitutes the complete Ohm's law. Let $\mathbf{V}_{e}$ be the electron macroscopic (fluid) velocity, whereas $\mathbf{V}_{i}$ represents the ion macroscopic velocity. Therefore, the current density is

$$
\mathbf{J}=n_{e} q\left(\mathbf{V}_{i}-\mathbf{V}_{e}\right)
$$

The electron number density is $n_{e}$ (the same as the ion number density for atomic number $\Xi=1$, which is assumed here), the electron's charge is $-q$, and the mass of a single electron is $m_{e}$. The fluid-momentum equation of electrons in the lab frame then reads, in mks units,

$$
\begin{aligned}
\partial_{t} \mathbf{V}_{e}+\mathbf{V}_{e} \cdot \nabla \mathbf{V}_{e}+ & \left(\frac{1}{m_{e} n_{e}}\right) \nabla P_{e} \\
& =-v_{e}\left(\mathbf{V}_{e}-\mathbf{V}_{i}\right)-\frac{q}{m_{e}}\left(\mathbf{E}+\mathbf{V}_{e} \times \mathbf{B}\right)
\end{aligned}
$$

Here, $P_{e}$ is the electron pressure, and $v_{e}$ is the momentumtransfer collision frequency of an average electron with the ions. As usual, the latter effect is represented as a friction. The electron collision frequency can be either the classical coulomb collision frequency, or it can be anomalous because of the activity of microturbulence. The leftmost term in Equation (36) vanishes in the assumed steady state, and the inertial $\left[\mathbf{V}_{e} \cdot \nabla \mathbf{V}_{e}\right]$ term generally can be neglected in comparison to the electron-pressure gradient when the electron macroscopic velocity $\mid \mathbf{V}_{e}$ is small compared to the electron thermal velocity $\left[2 T_{e} / m_{e}\right]^{1 / 2}$. That inequality is well satisfied at the postulated temperatures.

Accordingly, by neglecting the two leftmost terms, multiplying Equation (36) by $m_{e} / q_{e}$, and using Equation (35), Equation (36) becomes

$$
\mathbf{E}+\mathbf{V}_{i} \times \mathbf{B}=\left(\frac{m_{e} v_{e}}{q}\right)\left(\frac{\mathbf{J}}{n q}\right)+\left(\frac{\mathbf{J}}{n q}\right) \times \mathbf{B}-\left(\frac{1}{n q}\right) \nabla P_{e}
$$

A more familiar form is

$$
\mathbf{E}+\mathbf{V}_{i} \times \mathbf{B}=\eta \mathbf{J}+\left(\frac{1}{n q}\right) \mathbf{J} \times \mathbf{B}-\left(\frac{1}{n q}\right) \nabla P_{e}
$$

The left-hand side is the electric field in the moving frame ( $\mathbf{E}$ is the electric field in the lab frame), and $\eta$ is the fundamental resistivity of plasma, $\eta=\sigma^{-1}$. The electrical conductivity of plasma is $\sigma=n q^{2} / m_{e} v_{e}$. The second term on the right, involving $\mathbf{J} \times \mathbf{B}$, is the magnetic Hall term. Other terms 
in Equation (37b) that go beyond the simple Ohm's law are the motional electromotive force due to plasma rotation, which is part of the $\mathbf{V} \times \mathbf{B}$ term, and the thermal Hall term, which is the electron-pressure gradient term. The thermal force term arising from the specific nature of coulomb collisions of charged particles (Ref. 9) is neglected here in the interest of simplicity. It can easily be included if so desired.

In this section, we want to emphasize that the effective electrical conductivity is not necessarily the fundamental conductivity $\sigma$. Rather, the effective electrical conductivity generally depends on the Hall terms, on whether rotation occurs, and especially on the global electrical boundary conditions that are present (either in a simulation or an experiment). Concomitantly, we shall illustrate that the nature of the plasma-field mixing layer and its spatial rate of broadening depends on the possibility of plasma rotation, on the presence of an electron pressure gradient, and on the global electrical boundary conditions. In order to demonstrate the role of the Hall effect in a simple manner, we first neglect rotation and electron pressure. They are reinserted later.

\subsubsection{No Plasma Rotation, No Electron Pressure}

It is apparent that

$$
\left(\frac{\sigma B}{n q}\right)=\frac{\omega_{c e}}{v_{e}}=\Omega
$$

wherein $\omega_{c} e$ is the electron cyclotron frequency, and $\Omega$ is the conventional Hall parameter. Recall that $\Omega \gg 1$ in the plasma's edge layer with magnetic field in the parameter regime of interest. Then multiplying Equation (37b) by the conductivity $\sigma=n_{e} q_{e}^{2} / m_{e} v_{e}$ is seen to be equivalent to

$$
\mathbf{J}+\mathbf{\Omega} \mathbf{J} \times \hat{\mathbf{b}}=\sigma\left(\mathbf{E}+\mathbf{V}_{i} \times \mathbf{B}\right)
$$

where $\hat{\mathbf{b}}$ is the unit vector along $\mathbf{B}$. The Hall term is potentially very important in the considered applications, since $\Omega$ is very large in the plasma-field mixing layer, on the order of 400 for hydrogen propellant and 560 for helium propellant in Table I. Equation (39) is now solved for the current density to exhibit the effective electrical conductivity.

The azimuthal component of Equation (39) is

$$
J_{\theta}-\Omega J_{x}=-\sigma V_{x} B
$$

where it is noted that, in an axially symmetric steady state in the lab frame, the azimuthal electric field $E_{\theta}=0$. The condition on $E_{\theta}$ results from the integral form of Faraday's law of induction, namely $\nabla \times \mathbf{E}=-\partial_{t} \mathbf{B}$. The component of Equation (39) normal to the flux surfaces (the $\hat{\mathbf{x}}$-component) is

$$
\Omega J_{\theta}+J_{x}=\sigma E_{x}
$$

It has been noted that rotation is precluded here. It also has been implicitly assumed that there is no azimuthal magnetic field. (The relevance of the azimuthal magnetic field to the considered magnetic nozzle configuration will be brought out later. See Appendix D.)

Upon multiplication of Equation (41) by $\Omega$ and adding to Equation (40), one finds

$$
J_{\theta}=\left(\frac{\sigma}{1+\Omega^{2}}\right)\left(\Omega E_{x}-V_{x} B\right)
$$

Now in the limit $\Omega \ll 1$ (the electron gyrofrequency much smaller than the electron collision frequency), Equation (42) reduces to a form of Equation (13), the simple Ohm's law:

$$
J_{\theta}=-\sigma V_{x} B
$$

The remainder of this subsection shows how the simple Ohm's law for azimuthal current conduction still can be valid in spite of the presence of a strong longitudinal magnetic field, $\Omega \gg 1$. Two extreme examples will be discussed to illustrate the importance of the Hall effect together with global boundary conditions. The first example is that the Hall current is absolutely not allowed to flow because of global electrical boundary conditions imposed upon the system. The second one is at the opposite extreme: namely, the Hall voltage is completely shorted out by another kind of those boundary conditions, and the Hall current then flows unimpeded. (Of course, there could be a number of intermediate possibilities, any one of which may be relevant to a particular experiment or simulation.)

2.3.1.1 No Hall current allowed.-If absolutely no current is allowed to flow across the flux surfaces (no quasiradial currents) because of insulating boundary conditions at the wall (and the backplate in the breech of the nozzle), then $J_{x}=0$ and Equation (40) again yields the simple Ohm's law, exactly in the form of Equation (43) above. This result is obtained in spite of a formally huge magnetic Hall term in Equation (39). On the other hand, motivated by the huge Hall parameter $\Omega$, it is prudent to be mindful, both in experiment and numerical simulation, as to whether any quasi-radial Hall currents are inadvertently allowed to flow.

Of course, the coaxial wall (which is the field coil set) has to be metallic so as to support and shape the magnetic nozzle field. However, that requirement need not conflict with the suppression of Hall currents because the conducting wall can be covered with a thin layer of insulating material. (Such a composite boundary condition then should be properly represented in numerical simulations.) Nevertheless, the presence of the insulating wall layer does not necessarily suppress the Hall current. A conducting plasma layer could 
form along the wall in a real experiment and thus could support a closure path for the Hall current.

In the present subcase it is instructive to examine the Hall electric field that corresponds to complete suppression of the Hall current. If no quasi-radial currents can flow, Equation (41) indicates that the associated space-charge field (the Hall field) is given by $E_{\mathrm{Hall}}=(\Omega / \sigma) J_{\theta}$. It is instructive to write this in reverse, utilizing Equation (38), as

$$
J_{\theta}=\left(\frac{\sigma}{\Omega}\right) E_{\text {Hall }}=\frac{n q E_{\text {Hall }}}{B}
$$

In the given circumstance of no Hall current, a Hall electric field builds up across the flux surfaces to just such a value that the azimuthal " $E / B$ " (ratio of of electric to magnetic field strengths) drift velocity exactly satisfies the simple Ohm's law. The effective electrical conductivity then is just the fundamental conductivity $\sigma$, which is the same as if there were no magnetic field. The Hall electric field is a spacecharge field associated with surface charges that are built up on insulating surfaces that stop the flow of the Hall current.

The $E / B$ drift velocity referred to above must be the azimuthal guiding-center drift velocity of the electrons. Although it is true that the ions have exactly the same $E / B$ guiding-center drift velocity, such an ion drift is not effective in canceling the electron current for the following reason: it has been assumed here at the outset that the ion fluid has no macroscopic rotational velocity; hence, within the present model the ion fluid is incapable of providing an azimuthal current to cancel that of the electrons. The physics that makes this consistent is that there are other ion drifts besides the $E / B$ guiding center drift. For example, there is the $\nabla B$ drift, and there is the diamagnetic drift resulting from a density gradient, which means that there is a lack of cancellation of neighboring gyro-orbits.

In order that the ion fluid not rotate, which was the present assumption, it must be the case that the azimuthal $E / B$ guiding center drift velocity of the ions is canceled by other effects, such as ion $\nabla B$ drifts or ion magnetization currents. It does not really matter what the other effects are. All that matters is the assumption that such individual particle mechanisms add up to constitute an ion fluid that does not rotate. It will be seen later that the assumption of no ionfluid rotation is indeed consistent with the companion assumption that the Hall current cannot flow.

2.3.1.2 Hall current allowed (voltage shorted out).Suppose the Hall electric field is completely shorted out $\left(E_{\text {Hall }}=0\right)$, and the Hall current (the quasi-radial current $J_{\text {Hall }}$ is perfectly free to flow. Note that plasma rotation is still assumed to be absent. The assumption of no macroscopic mass rotation is not generally consistent with the presence of Hall current in the axially symmetric configuration of the magnetic nozzle, but could be made consistent in a simulation. Also, it could represent a physically realistic situation within a limited region upstream, before the new ion fluid has had a chance to be spun up by the $\mathbf{J}_{\text {Hall }} \times \mathbf{B}$ magnetic body force of the Hall current.

A concrete example of shorting out the Hall electric field would be the use of a noninsulated metallic backplate in the breech of the nozzle. Conduction in the metallic backplate, for example a copper plate, is unaffected by the embedded magnetic nozzle field because of the very large collision frequencies (with phonons) of the metallic conduction electrons, which, however, also have a very high density that produces a high conductivity. If high-conductivity longitudinal electron currents (along the magnetic field lines) were to be allowed access to the backplate, then it would have the effect of setting all flux surfaces at practically the same potential, thus shorting out the radial space-charge field. Such a condition, which allows the radial Hall current to flow unimpeded, implies that either the uninsulated coaxial wall or an edge layer of conducting plasma is available at large radius to provide a longitudinal current return path to the backplate. If there were no such return path, then either net charge would be continually removed from the system or bipolar space-charge electric fields would continually build up, neither of which is tenable within the steady-state context.

In the present subcase, in which the Hall electric field $E_{\text {Hall }}$ is assumed to be completely shorted out, Equation (42) reduces to

$$
J_{\theta}=-\frac{\sigma V_{x} B}{1+\Omega^{2}}
$$

The Hall effect, in conjunction with the assumed boundary conditions and in the absence of plasma rotation, has reduced the effective electrical conductivity by a huge factor; $\sigma_{\text {eff }}=\sigma /\left(1+\Omega^{2}\right)$. For the parameters of interest, this reduction factor is on the order of $10^{5}$.

The physical basis for this reduced conductivity, termed "magnetoresistance," has an origin that is somewhat complicated. In view of the present assumption that the ions have no fluid velocity in the azimuthal direction, the burden of carrying the azimuthal current falls on the electrons. Without the " $E_{\mathrm{Hall}} / B$ " drift velocity induced by the Hall field (as in the preceding subcase), an electron can only undergo a displacement at each collision by approximately a tiny gyroradius step across the magnetic field. This displacement is in the azimuthal direction, in response to the azimuthal applied electric field $E_{\theta \text { app }}=-V_{x} B$. It can be shown that the electron's effective gyroradius must be small, on the order of $E_{\theta}$ app $/ B \omega_{c e}$. This particular gyroradius is much smaller than the electron's thermal gyroradius. Hence, the effective step size involved in the azimuthal electrical conductivity of electrons across the magnetic field is even smaller than the electron's thermal gyroradius. In contrast, the null-magnetic-field conductivity is based upon a much larger step size; namely $\lambda_{e}$. 
When the Hall voltage is shorted out, thereby precluding the $E_{\mathrm{Hall}} / B$ drift, it is the very small step size in the azimuthal direction at a collision of an electron that proves to be responsible for the decreased electrical conductivity. This phenomenon is termed "magnetoresistance." The details of the three-dimensional scattering of the cycloidal electron orbits underlying magnetoresistance lie beyond the purview of this report.

The minimal intermixing of plasma and magnetic field, found earlier for the case of classical resistivity, was based upon the applicability of the simple Ohm's law. In the present context, that result requires that no Hall current be allowed to flow. However, if the Hall electric field were even partially shorted out, Hall current would flow, and a concomitant drastic reduction in effective electrical conductivity would ensue, thereby enhancing the plasma-field intermixing.

Clearly, it is important for the elucidation of plasma-field intermixing in the magnetic nozzle to ascertain the boundary conditions and Hall-return-current paths in the envisioned magnetic nozzle device or experiment, as well as in numerical simulations of plasma flow through magnetic nozzles. Attention also should be directed to whether plasma rotation is allowed in the simulations. Also, computer simulations are vulnerable to subtle numerical effects that impact the computed physics. It is conceivable that these might inadvertently allow such Hall currents to intrude. Thus, any observed deviation in the magnetic nozzle device or in its numerical simulation that allows even some Hall current may signify a partial shorting of the Hall voltage. The question should then be raised regarding the effective electrical conductivity that governs diffusive intermixing of plasma and magnetic field.

It is instructive at this point to examine the Hall current with completely shorted Hall field. From Equations (41) and (42), with $E_{\text {Hall }}=0$ (Hall field shorted out), and in the limit of large $\Omega$, one finds with the help of Equation (38) that

$$
J_{\mathrm{Hall}}=n_{e} q V_{x}
$$

Note that the velocity $\mathbf{V}$ represents the massive plasma velocity, not the electron velocity. Thus, the Hall current is carried across flux surfaces by ions. Electrons remain relatively attached to the flux surfaces in regard to their radial motion, in the limit of large $\Omega$ and concomitantly small electron collision frequency $v_{e}$. Since the space-charge field is assumed to be globally shorted out, quasi-neutrality is not enforced in the conventional manner. Electrons vacate the local region at the same rate as the ions by flowing away along the longitudinal magnetic field.

Note also that the above result for $J_{\mathrm{Hall}}$ can be written as follows:

$$
\left.\mathbf{J}_{\text {Hall }}=n_{e} q(\mathbf{V} \times \mathbf{B}) \times \frac{\mathbf{B}}{B^{2}} \text { (radial x-component }\right)
$$

This form of expression for the quasi-radial Hall current indicates that it is associated with a guiding-center drift velocity of the ions $E_{\theta}$ app $B$ in the applied electric field, $\mathbf{E}_{\boldsymbol{\theta} \text { app }}=\mathbf{V} \times \mathbf{B}$. A stationary magnetoplasma placed in an externally applied electric field would acquire a Hall current of this form when the Hall voltage is shorted out.

Obviously, electrons connecting longitudinally along magnetic flux to a conducting backplate could serve to provide part of a current-return path for ions that connect transversely across flux surfaces to the conducting coaxial wall or to a conducting low-density plasma layer along the wall. If such a partial current path were fully completed along the wall, the space-charge field (Hall field) that otherwise enforces quasi-neutrality in the radial direction would be shorted out. Instead, quasi-neutrality would then be maintained by having the electrons and the ions vacate a given position at the same rate; however, they would flow out in their different directions, longitudinal versus radial.

With regard to such a closure path for the Hall current, in terms of classical (Spitzer) resistivity, the longitudinal electrical conductivity in a plasma-wall layer would be rather insensitive to the density of plasma in that layer (Ref. 10). It is important to note that such a path-closure process with longitudinal electron flow is implicitly assumed in the above result for the ions as carriers of the radial Hall current. Otherwise, powerful radial space-charge electric fields would cause electrons to move across flux surfaces with the ions to preserve quasi-neutrality. Examples of such powerful spacecharge fields are given in a later discussion in the beginning of Section 3.0, "Resistivity From Gradient-Driven Microinstabilities."

As already mentioned, a highly conducting backplate would practically set all flux surfaces at the same potential, thus shorting out the Hall electric field. But even if the conducting backplate were rendered inaccessible to electrons by a coating of insulating material, an alternative Hall-current return path might become available, such as a layer of lowtemperature plasma next to the insulated backplate. A lowtemperature plasma-wall layer would raise the electron collision frequency up to near the electron cyclotron frequency. A $50 \mathrm{eV}$ in hydrogen plasma dropping to 1 or $2 \mathrm{eV}$ in the plasma layer at the backplate would accomplish similar results. The possibility then arises of electron conduction currents flowing radially outwards across flux surfaces that are intersecting the backplate. Although the electrical conductivity of such an electron-shorting path $\left(\sim 10^{3} \mathrm{mho} / \mathrm{m}\right)$ would be quite small in comparison to metallic conductivity $\left(\sim 10^{7} \mathrm{mho} / \mathrm{m}\right)$, it would still be many orders of magnitude larger than the conductivity of any insulator. Since the full effect on the conductivity with unimpeded Hall current is a huge effect, even an imperfect shorting path may make a meaningful difference. Moreover, ions within the plasmawall layer next to the backplate could also contribute to path closure by crossing the flux surfaces there, traveling radially inwards. 
It is important to remark that having longitudinal Hallcurrent closure along magnetic nozzle field lines necessitates allowing for azimuthal magnetic field components. Longitudinal currents in axially symmetric configurations generate azimuthal magnetic fields according to the integral form of Ampere's law: $\nabla \times \mathbf{B}=\mu_{0} \mathrm{~J}$. In Appendix $\mathrm{D}$ the subject is briefly addressed, while also allowing for rotation of plasma. It is shown there that the consequent azimuthal magnetic field component $B_{\theta}$ has the order-of-magnitude $\beta_{i} B$, where $\beta_{i}$ is the ratio of ion thermal pressure to magnetic pressure of the nozzle magnetic field, $\mathbf{B}$.

A complete model of propellant flow in a nozzle magnetic field, which embodies longitudinal currents and azimuthal magnetic fields as well as plasma rotation and electron pressure, is very complicated. It would be accessible to simulations that include Hall effects and that carefully treat global boundary conditions. The model is not extended in such a manner here, although some discussion of the extended model is offered in Appendixes D and E. However, a partial extension of the present model that is tractable and instructive is introduced in Section 2.3.2.2, "Hall current allowed (voltage shorted out)."

Thus, we shall complete this section on the Hall effect by including electron pressure and plasma rotation in the extension of Ohm's law. In the axially symmetric configuration, it will be seen that Hall current and plasma rotation must occur together. The partially extended model presented below, however, does preclude an azimuthal magnetic field, $\mathbf{B}_{\boldsymbol{\theta}}$. Nevertheless, it is found that allowing for the possibility of plasma rotation proves to have a profound effect upon the nature of the plasma-field mixing layer, when the Hall electric field is shorted out.

\subsubsection{Plasma Rotation and Electron Pressure}

In the above discussion, the azimuthal component of the plasma fluid momentum equation has been ignored. This restriction would apply to a simulation that had provision only for radial and axial components of propellant velocity. To be consistent, as shown below, such a code also should have hard-wired into it the condition that the Hall current automatically be forbidden. In the above discussion, however, rotation was automatically excluded but the Hall current was not automatically excluded. In a real magnetic nozzle experiment or device, the occurrence of propellant rotation and Hall current would be coupled.

These remarks are illustrated by the azimuthal component of the plasma momentum equation for the case of an axially symmetric steady-state configuration.

$$
\rho(\mathbf{V} \cdot \nabla \mathbf{V})_{\theta}=(\mathbf{J} \times \mathbf{B})_{\theta}
$$

Upon working out the details, one finds

$$
\left[\left.\frac{\rho}{r(l)}\right|_{\rfloor} \mathbf{v} \cdot \nabla\left[r(l) V_{\theta}\right]=\left[\left.\frac{\rho V_{l /}}{r(l)}\right|_{\rfloor} \partial_{l}\left[r(l) V_{\theta}\right]=-J_{\text {Hall }} B\right.\right.
$$

Here $V_{\theta}$ is the rotational velocity of plasma, $V_{/ /}$is the velocity of plasma along the field line, $l$ measures length along a field line, and the $x$-direction (normal to the flux surfaces) pertains to $J_{\text {Hall }}$. The magnetic field component $B_{\theta}$ has been ignored.

It is important to note that the radius variable $r(l)$ is a function of $l$ that characterizes the geometry of the magnetic nozzle field. Thus $r(l)$ constitutes the distance from the axis of symmetry to a considered point a distance $l$ along a given field line, as measured from the breech. Strictly speaking, the spatial derivative in Equation (49) is along the r,Z-projection of a propellant streamline, since the $\theta$-coordinate is in the ignorable direction. In principle, the shape of the field line $r(l)$ will be somewhat distorted from its vacuum-field shape by the plasma propellant and must be determined selfconsistently with the propellant flow, as is done in MHD simulations such as MACH2. However, the Hall effects were excluded from the MACH2 simulation in Reference 2.

It is important to remark that if the Hall current density $J_{\mathrm{Hall}}$ is everywhere forbidden by external boundary conditions, then Equation (49) implies that there can be no rotation of plasma, provided that injected plasma is inserted with axial symmetry into the breech and has no net injected macroscopic rotation at any radius. Conversely, if it is known that there is no macroscopic rotation anywhere, then Equation (49) implies that there also can be no Hall current. Simulations that are without access to the azimuthal momentum equation of the propellant then lose that means of enforcing consistency between the presence of the Hall current and the presence of rotation.

It is also important to note that even in the event of Hall currents and concomitant rotation of plasma, the rotational kinetic energy can be recovered downstream for conversion into axially directed thrust. This conversion can be effected in the diverging region of the nozzle where presumably $J_{\text {Hall }}$ approaches 0 . Reduction of temperature downstream increases $v_{e}$ relative to $\omega_{c e}$, making Hall effects less important downstream. From Equation (49) above, the rotational velocity then vanishes as $[r(l)]^{-1}$. The conversion of rotational kinetic energy into longitudinal kinetic energy is a consequence of conservation of total energy. This feature can be demonstrated for axially symmetric steady flow by means of the generalized Bernoulli equation including rotation, which describes energy balance along the streamline projections in the meridian plane.

We now invoke Ohm's law including rotation and electron pressure. Define an effective electric field $\mathbf{E}_{\text {eff }}$ such that

$$
\mathbf{E}_{\text {eff }}=\mathbf{E}+\mathbf{V} \times \mathbf{B}+\left(\frac{1}{n q}\right) \nabla P_{e}
$$

Here, $\mathbf{E}$ is the electric field in the lab frame. Multiplying Equation (37b) by $\sigma$ and combining with Equation (38), the result is 


$$
\mathbf{J}+\mathbf{\Omega} \mathbf{J} \times \hat{\mathbf{b}}=\sigma \mathbf{E}_{\text {eff }}
$$

The azimuthal and Hall components of Equation (51) are, respectively,

$$
J_{\theta}-\Omega J_{\text {Hall }}=\sigma E_{\text {eff } \theta}
$$

and

$$
\Omega J_{\theta}+J_{\text {Hall }}=\sigma E_{\text {eff } x}
$$

Now $E_{\theta}=0$ in an axially symmetric steady state in the lab frame, and there is likewise no azimuthal pressure gradient $\nabla_{\theta} P_{e}=0$. Therefore, from Equation (50),

$$
E_{\text {eff } \theta}=-V_{x} B
$$

Also,

$$
E_{\text {eff } x}=E_{\mathrm{Hall}}+V_{\theta} B+\left(\frac{1}{n q}\right) \partial_{x} P_{e}
$$

2.3.2.1 No Hall current allowed.-In this case, $J_{\text {Hall }}=0$, so that Equations (52a) and (53a) produce $J_{\theta}=-\sigma V_{x} B$, which is again the simple form of Ohm's law. Thus, the results obtained earlier for the plasma-field mixing layer width are again realized even when plasma rotation and electron pressure are not precluded from the model, as long as the Hall current is strictly forbidden by the global electrical boundary conditions. Moreover, when the Hall current is forbidden, so is plasma rotation, as discussed above.

Electron pressure plays a new role, though, as can be seen from Equations (52b) and (53b), which now yield, with the help of Equation (38)

$$
J_{\theta}=n q\left(\frac{E_{\mathrm{Hall}}}{B}\right)+\left(\frac{1}{B}\right) \partial_{x} P_{e}
$$

In this case, the electron-fluid pressure-gradient term $B^{-1} \partial_{x} P_{e}$ contributes to the azimuthal current density as well as the $E / B$ drift. The second term of Equation (54) is partially caused by a lack of cancellation of azimuthal current elements from neighboring electron gyro-orbits, due to the spatial gradient of electron density in the $\mathrm{x}$-direction (normal to flux surfaces). From the manner in which the fluid equations are derived from more detailed kinetic models, the electron pressure gradient in the fluid model also automatically incorporates other individual-particle contributions, namely, guiding center drifts. For brevity, the $B^{-1} \partial_{x} P_{e}$ term is henceforth referred to as "magnetization current."

Although the $E / B$ guiding-center drift velocity of the ions equals that of the electrons, seemingly canceling that contribution to the electron current, it is also true-in this subcase - that there is no net rotation of the ion fluid. The "ion-fluid momentum" is the same as the "plasma-fluid momentum." The ion fluid cannot produce any macroscopic contribution to azimuthal current in the absence of propellant-fluid rotation, as pointed out earlier. Thus, on the basis of the azimuthal momentum equation of the plasma when the Hall current is forbidden, the $E / B$ drift velocity of the ions must be canceled by other individual-particle ion effects, such as other guiding-center drifts and magnetization currents. Therefore, the $E / B$ drift velocity in Equation (54) must refer to the guiding-center drift velocity of the electrons only, it being understood that there is no net rotational contribution from the ions when $J_{\mathrm{Hall}}=0$.

2.3.2.2 Hall current allowed (voltage shorted out).- - In the extreme case in which $E_{\mathrm{Hall}}=0$, concomitant with the existence of current-return paths sufficient to provide a complete circuit for the Hall current, the solution for the azimuthal current density $J_{\theta}$ from Equations (52a) and (52b) reads as follows: Using Equations (53a) and (53b), one finds

$$
J_{\theta}=\frac{\sigma}{\left(1+\Omega^{2}\right)}\left\{-V_{x} B+\Omega\left[V_{\theta} B+\left.\left(\frac{1}{n q}\right) \partial_{x} P_{e}\right|_{\mid} ^{\top \|}\right\}\right.
$$

In the limit of large $\Omega$, with help of Equation (38), this expression reduces to

$$
J_{\theta}=-\left(\frac{n q V_{x}}{\Omega}\right)+n q V_{\theta}+\left(\frac{1}{B}\right) \partial_{x} P_{e}
$$

It will be seen shortly that over most of the length of the nozzle, $V_{\theta} \gg V_{x}$ in the plasma-field mixing layer between plasma and magnetic field. Moreover, $\Omega \gg>1$ there. Hence the azimuthal current carried by the plasma in the plasmafield mixing layer essentially contains two principal contributions, one from the ion-fluid rotation, and the other from the electron magnetization current.

Next, we calculate the Hall current density for large $\Omega$, from Equations (52b), (53b), and (56):

$$
\begin{aligned}
J_{\text {Hall }} & =-\Omega J_{\theta}+\sigma E_{\text {eff } x} \\
& =n q V_{x}-\Omega n q V_{\theta}-\left(\frac{\Omega}{B}\right) \partial_{x} P_{e}+\sigma\left[V_{\theta} B+\left.\left(\frac{1}{n q}\right) \partial_{x} P_{e}\right|_{]}\right. \\
& =n q V_{x}
\end{aligned}
$$

The last step is obtained by invoking Equation (38). Thus, in the approximation $\Omega^{2} \gg 1$, it would appear that ions still carry the Hall current across flux surfaces, when rotation and electron pressure are included in the model. However, this 
result is misleading because Equation (56) is not completely accurate, being only the leading approximation (for small $\Omega^{-1}$ ). The leading approximation to $J_{\theta}$, in terms of $\Omega^{-1}$, is prone to error if $J_{\theta}$ is multiplied by large $\Omega$, as in the above equation for $J_{\text {Hall }}$. The exact answer, using the exact Equation (55) instead of Equation (56), and again invoking Equations ( 38$)$ and $(53 \mathrm{~b})$, proves to be

$$
J_{\text {Hall }}=\left(\frac{\Omega^{2}}{1+\Omega^{2}}\right) n q V_{x}+\left(\frac{\Omega}{1+\Omega^{2}}\right)\left[n q V_{\theta}+\left.\left(\frac{1}{B}\right) \partial_{x} P_{e}\right|_{]}\right.
$$

Now finally taking the limit of large $\Omega$, this reduces to

$$
J_{\mathrm{Hall}}=\left(1-\frac{1}{\Omega^{2}}\right) n q V_{x}+\left(\frac{1}{\Omega}\right)\left[n q V_{\theta}+\left.\left(\frac{1}{B}\right) \partial_{x} P_{e}\right|_{]}\right.
$$

Thus, the quasi-radial Hall current is only partly carried directly by ions across flux surfaces. Because of subtle magnetic effects, it also contains contributions from the ion-fluid rotation and from the azimuthal electron magnetization current. This is true only in regions that the plasma has been able to reach. The final results will indicate that $V_{\theta} / \Omega$ is indeed of the same order of magnitude as $V_{x}$.

It will be seen that the ions do not travel very far radially from the core plasma during times of interest. Although the Hall current circuit could be completed near the core's edge, the presence of an inadvertent halo of low-density plasma could as well facilitate a more distant closure of the Hall path. Nevertheless, the inadvertent presence of a pervasive low-density halo plasma might allow longitudinal completion of the Hall current circuit out near the wall, and across a conducting backplate.

Now safely invoking Equation (56) within this higher order calculation in $\Omega^{-1}$, Equation (59) becomes, in leading order,

$$
J_{\text {Hall }}=n q V_{x}+\frac{J_{\theta}}{\Omega}
$$

This result also is in direct agreement with Equation (52a), whose meaning is now clarified.

With the help of Equations (56) for $J_{\theta}$ and (60) for $J_{\text {Hall }}$, both transverse components of the plasma momentum equation can be utilized to find $V_{\theta}$ and $V_{x}$. An inversion in the procedure proves convenient relative to the simple case of a resistive plasma-field mixing layer. Now the quasi-radial momentum equation determines the rotational velocity, whereas the azimuthal momentum equation determines the quasi-radial velocity. These results are then used to examine the evolution of the plasma-field interface layer thickness.
The qualitative conclusion follows. If the Hall voltage is shorted to allow Hall current in the presence of selfconsistent rotation and electron pressure, the plasma-field mixing layer thickness between propellant and magnetic field becomes very different from that obtained using the simple resistive Ohm's law.

To carry out this calculation, we first utilize the plasma momentum equation in the direction normal to the flux surfaces, the x-direction (as usual, it is assumed that the transverse pressure gradient dominates the transverse inertial effects). This equation reads

$$
\partial_{x} P_{\text {tot }}=J_{\theta} B \approx n q V_{\theta} B+\partial_{x} P_{e}
$$

In the second part of Equation (61), we made use of Equation (56), wherein the small $V_{x} / \Omega$ term was neglected in comparison to $V_{\theta}$. Since the total plasma pressure is $P_{\text {tot }}=P_{i}$ $+P_{e}$, the second part of Equation (61) is equivalent to

$$
\left(\frac{1}{B}\right) \partial_{x} P_{i} \approx n q V_{\theta}
$$

Therefore, the macroscopic ion-fluid rotation is actually seen to be effected by the ion magnetization current, to leading order in $\Omega^{-1}$.

Next, Equations (61) and (62) are input to Equation (49), the azimuthal component of the plasma momentum equation. This is done as follows: Integrate Equation (49) along a given representative field line, from the breech region over an indefinite length $L$, which is a significant fraction of the length of the nozzle. The present model is valid while Hall effects are dominant, which means that the temperature is yet large enough that $\omega_{c e}>v_{e}$. The result is

$$
\left(r V_{\theta}\right)_{L}=-\int_{0}^{L}\left(\frac{r J_{\mathrm{Hall}} B}{\rho V_{/ /}}\right) \mathrm{d} l
$$

Quantitative numerical results would require carrying out the indicated integration along a selection of representative field lines near and in the plasma-field mixing layer, with their self-consistent field-line shapes characterized via the function $r(l)$. Instead, we make the following approximation, in the spirit of obtaining an order-of-magnitude scaling result for the plasma-field mixing layer width: The integral is approximated as the average value of the integrand, say, near the throat, multiplied by the distance $L$. Then, suppressing moderate numerical distinctions between the values of quantities at $l=\mathrm{L}$ and their representative average values, we multiply Equation (63) by the quasi-neutral ion number density multiplied by charge, $n_{i} q$, to obtain 


$$
n_{i} q V_{\theta} \approx-L\left[\left(\frac{n q B}{\rho V_{/ /}}\right) J_{\text {Hall }}\right]
$$

Here, for example, the numerical ratio of the average value of $r$ along a field line to $r(L)$ has been suppressed.

Note that the composite ratio $n_{i} q B / \rho$ is just the ion cyclotron frequency $\omega_{c i}=q B / m_{i}$. Accordingly, we define a large parameter $\Lambda \gg>1$ by

$$
\Lambda \equiv \frac{\omega_{c i} L}{V_{/ /}}
$$

Now $V_{/ /}$is closely coupled by the Bernoulli equation to $\left[\mathrm{\gamma}\left(T_{i}+T_{e}\right) / m_{i}\right]^{1 / 2}$, the speed of sound, which also is near the ion thermal velocity. It follows that the parameter $\Lambda$ has the order of magnitude of the ratio of nozzle length to ion gyroradius. To within a trivial numerical factor,

$$
\Lambda \approx \frac{L}{a_{i}}
$$

Therefore, Equation (64) is rewritten, using Equations (60) and (65), as

$$
n q V_{\theta} \approx-\Lambda J_{\text {Hall }}=-\Lambda\left(n q V_{x}+\frac{J_{\theta}}{\Omega}\right)
$$

Here, it is important to note, in view of Equation (62), that the Hall current $J_{\mathrm{Hall}}$ exists in virtue of the ion pressure gradient, which creates the ion-fluid rotation. This is provided that the Hall electric field $E_{\text {Hall }}$ is shorted out. Recall that $\Omega=\omega_{c e} v_{e}$, where $\omega_{c} e$ is the electron gyrofrequency, and $v_{e}$ is the collision frequency of an average electron with ions.

The quasi-radial velocity $V_{x}$ is solved as follows: Combining the left-hand side of Equation (67) with Equation (62), and substituting Equation (61) for $J_{\theta}$ on the right-hand side, the resulting equation for $V_{x}$ can be written as

$$
n q V_{x} \approx-\left[\left(\frac{1}{\Lambda B}\right) \partial_{x} P_{i}+\left.\left(\frac{1}{\Omega B}\right) \partial_{x} P_{\text {tot }}\right|_{]}\right.
$$

In the parameter regime of interest, $\Lambda$ and $\Omega$ are large and of the same order, and the two pressure gradients also are of the same order of magnitude. It is implicitly assumed that electrons and ions enter the breech with practically the same temperature, and also the same density profiles because of the quasi-neutrality condition. Therefore, this equation essentially constitutes a verification that $V_{x}$ is indeed very small compared with $V_{\theta}$. (See Eq. (62).) Now referring back to Equation (67), note that all terms therein are of the same order of magnitude when $\Lambda$ is multiplied through on the right-hand side.

Although not actually necessary, the following discussion is simplified by setting the electron pressure approximately equal to the ion pressure, within the plasma and within the plasma-field mixing layer: $P_{e} \approx P_{i}$. Then Equation (68) reads

$$
\left.n e V_{x} \approx-\frac{1}{\Lambda}\left[1+\left(\frac{2 \Lambda}{\Omega}\right)\right]\right]\left[\left.\left(\frac{1}{B}\right) \partial_{x} P_{i}\right|_{]}\right.
$$

It is understood that Equation (69) is not necessarily an accurate quantitative statement, but rather represents a qualitative scaling result. In that sense, all quantities therein are meant to be representative average values when following the flow of a piece of propellant along a given magnetic field line.

Equation (69) can be utilized to estimate the plasma-field mixing layer width $\delta$ at the plasma-field interface. The basic modeling approximation, which also was employed earlier in the case of resistive diffusion, is to associate $V_{x}$ with $\mathrm{d} \delta / \mathrm{d} t$, the rate of increase of the plasma-field mixing layer width as the longitudinal flow is followed downstream:

$$
V_{x} \approx \frac{\mathrm{d} \delta}{\mathrm{d} t}
$$

The other part of the basic idea, also employed earlier, is to represent the ion pressure gradient within the plasma-field mixing layer by its value within the core plasma divided by the width of the plasma-field mixing layer:

$$
\partial_{x} P_{i} \approx-\frac{P_{i}}{\delta}
$$

Then, Equation (69) can be expressed as follows, where $T_{i}$ refers to the ion temperature and where $V_{\mathrm{th} i}^{2}=2 T_{i} / m_{i}$ is the square of the ion thermal velocity:

$$
\frac{\mathrm{d}\left(\delta^{2} / 2\right)}{\mathrm{d} t}=\frac{1}{\Lambda}\left[1+2\left(\frac{\Lambda}{\Omega}\right)\right] \frac{n T_{i}}{n q B}=\frac{1}{\Lambda}\left[1+2\left(\frac{\Lambda}{\Omega}\right)\right]\left(\frac{V_{\mathrm{th} i}^{2}}{2 \omega_{c i}}\right)
$$

It can be shown that the right-most factor $V_{\text {thi } i}^{2} / \omega_{c i}$ is insensitive to position along the nozzle.

One now integrates Equation (72) over time, while following the propellant downstream, assuming that the initial plasma-field mixing layer width of attached plasma in the breech is negligibly small. On the right-hand side, one 
simply takes average or representative values during transit of the length of the nozzle from breech to throat $L_{b t}$ and then finds

$$
\delta^{2} \approx \frac{1}{\Lambda}\left[1+2\left(\frac{\Lambda}{\Omega}\right)\right]\left(\frac{V_{\text {th } i}^{2}}{\omega_{c i}}\right)
$$

A rigorous approach requires integration over $L_{b}$ : $\mathrm{d} / \mathrm{d} t=V_{/ /} \mathrm{d} / \mathrm{d} L_{b t}$, noting that $\Lambda$ depends on $L_{b t} / V_{/ /}$and that $V_{/ /}$cancels in Equation (72). When $\Lambda<\Omega, \delta / a_{i}$ increases slowly with $L$, as $\left[\ln \left(L_{b t} / L_{b}\right)+\left(\delta_{b} / a_{i}\right)^{2}\right]^{1 / 2}$, where $L_{b}$ is a small starting length in the breech, and $\delta_{b}$ is an initial layer thickness derived later as $\delta_{b} \approx 2 a_{i}$.

For the transit time $t$, we invoke $t_{b t}=L_{b t} / V_{/ /}$, where $V_{/ /}$is a flow velocity in transit, and also note Equation (65) for $\Lambda$. Then Equation (73) becomes simply

$$
\delta^{2} \approx\left[1+2\left(\frac{\Lambda}{\Omega}\right) \frac{V_{\mathrm{th} i}^{2}}{\omega_{c i}^{2}}=\left[1+2\left(\frac{\Lambda}{\Omega}\right)\right] a_{i}^{2}\right.
$$

It is therefore seen from Equation (74) that when $\Lambda<\Omega$, the plasma-field mixing layer width is on the order of just a few ion gyroradii. This is a very favorable result in the sense that the width of the plasma-field mixing layer appears to be independent of the electron collision frequency, if the condition $\Lambda<\Omega$ is satisfied. On the other hand, when the opposite condition is satisfied, namely when $\Lambda \gg \Omega$, then a fundamental change occurs in the scaling of the boundary-layer width.

To examine this second possibility, note that $2(\Lambda / \Omega)$ has the following order of magnitude:

$$
2\left(\frac{\Lambda}{\Omega}\right) \approx 2\left(\frac{L_{b t}}{\lambda_{e}}\right) \sqrt{\frac{m_{e}}{m_{i}}}
$$

where $\lambda_{e}$ is recalled as the mean free path of electrons due to collisions (possibly anomalous) with ions. Also, $m_{e} / m_{i}$ is the electron-to-ion mass ratio. Thus, from Equation (74), if there are fewer electron mean free paths along $L_{b t}$ than $\sqrt{m_{i} / m_{e}}$, then the boundary-layer width at the plasma edge is just one ion gyroradius. However, if the characteristic length of the nozzle is substantially greater than that relative to $\lambda_{e}$, then from Equation (74) with $\Lambda \gg \Omega$,

$$
\delta^{2} \approx 2 a_{i}^{2}\left(\frac{L_{b t}}{\lambda_{e}}\right) \sqrt{\frac{m_{e}}{m_{i}}}
$$

It is satisfying to note that Equation (76) exactly reproduces Equation (28) for the case of simple resistive diffusion of plasma into field, without Hall current and without plasma rotation. It also is of interest to record the following equivalent form of Equation (76), representing the case of simple resistive diffusion:

$$
\delta^{2} \approx \frac{2 a_{i} L_{b t}}{\Omega}
$$

which is to be compared with $\delta^{2} \approx a_{i}^{2}$ when the nozzle's length is subcritical. Here, $\Omega=\omega_{c e} v_{e}$, where $\omega_{c e}=q B / m_{e}$ is the electron gyrofrequency, and $v_{\mathrm{e}}$ is the electron collision frequency.

To summarize, in a model that incorporates rotation, electron pressure, and Hall current unimpeded by a Hall voltage, we found a transition in the behavior of the boundary-layer width from the ion gyroradius to the width associated with simple resistive diffusion. The transition is realized provided that the nozzle is long enough. The critical length $L_{\text {crit }}$ is on the order of $\lambda_{e} \sqrt{m_{i} / m_{e}}$. In the case of classical resistivity, it can be shown that this quantity is essentially the same as $\lambda_{i}$ $\sqrt{m_{i} / m_{e}}$, which relates to $\lambda_{i}$, the mean free path for ion-ion coulomb collisions. The physical reason is that the plasma has spun up enough along its length in the edge layer so that the rotational velocity in the edge layer represents a motional electric field in the rotating frame, which is strong enough to suppress the outward quasi-radial flow of Hall current (simple resistive Ohm's law). To see this, note that the outward flow of Hall current produces a $\mathbf{J}_{\text {Hall }} \times \mathbf{B}$ force (for a magnetic field that points downstream), which is counterclockwise (looking downstream). Therefore, the $\mathbf{V}_{\text {rot }} \times \mathbf{B}$ component of the electric field in the rotating frame produces a quasi-radial component of the electric field that points inward, towards the plasma. If $\mathbf{V}_{\text {rot }}$ is large enough, this effect can suppress Hall current.

\subsubsection{Diffusion of Field Into Plasma With Hall Effect}

This section on the basic physics shall conclude with an account of external magnetic field diffusion into the interior plasma, when unimpeded Hall current and consequent plasma rotation are present, in a straight cylindrical geometry model of the core propellant. This complements the preceding treatment of protrusion of plasma into magnetic field, with Hall effect, as discussed in Section 2.3.2.2, "Hall current allowed (voltage shorted out)." Also, this treatment will thus generalize the earlier one that dealt with a planar interface, in Section 2.2.3, "Diffusion of Confining Magnetic Field." Such a magnetic-field diffusion process into the core plasma occurs at the same time as that of diffusion of the plasma into the external magnetic field. When the Hall current is totally suppressed by boundary conditions, the simple 
Ohm's law governs plasma-field intermixing, and both types of resistive diffusion act simultaneously to broaden the plasma-field mixing layer.

With Hall current (and rotation) present, a more complex version of Ohm's law is in force, together with the presence of an azimuthal magnetic field. This azimuthal field surrounds the longitudinal return current that replenishes the quasi-radial Hall current in a steady state. Under such circumstances, the diffusion of the nozzle magnetic field into plasma then becomes rather involved. Nevertheless, it is possible to develop a model of this process - diffusion of field into plasma - to the point that some of the principal features can be identified. In so doing, we shall assume axial symmetry and a steady state. Appendix D shows that the azimuthal field should be relatively small compared with the other components of the field.

The development begins with Faraday's law of induction in the lab frame:

$$
\nabla \times \mathbf{E}=-\partial_{t} \mathbf{B}
$$

According to the complete Ohm's law, this can be written as

$\nabla \times\left[\eta \mathbf{J}+\left(\frac{1}{n q}\right) \mathbf{J} \times\left.\mathbf{B}\right|_{\rfloor}-\nabla \times\left[\mathbf{V} \times \mathbf{B}+\left.\left(\frac{1}{n q}\right) \nabla P_{e}\right|_{\rfloor}=-\partial_{t} \mathbf{B}=0\right.\right.$

The right-hand side is zero in a steady state in the lab frame of reference.

For the $\mathbf{J} \times \mathbf{B}$ term in Equation (79), we use the momentum equation of the plasma. It is assumed that the electron and ion temperatures are uniform. Then, the plasma pressure gradient from the momentum equation is parallel to the plasma density gradient, so that the pressure gradient term does not survive the curl operator. Hence, only the inertial term appears from the momentum equation in connection with the $\mathbf{J} \times \mathbf{B}$ term. Similarly, the electron pressure gradient is parallel to the plasma density gradient. Then the electron pressure gradient term disappears from Equation (79).

We shall consider here only the case of classical Spitzer resistivity, which is insensitive to the electron number density and depends essentially only on the electron temperature, assumed uniform. Whether the final conclusions still would be valid for any anomalous (microturbulent) resistivity $\eta_{a}$ is an open question. In the case of resistivity arising from the LHD instability, an approximate scaling argument will be made later. The result is that after allowing for adiabatic longitudinal flow and for radial pressure balance in the plasma-field interface layer, $\eta_{a}$ is seen to depend only very weakly on density alone, as $n^{-1 / 6}$. This circumstance suggests that the arguments to be given here for classical resistivity would still hold for the LHD resistivity. This suggestion cannot be considered a rigorous conclusion, however, because $\eta_{a}$ actually proves to be profile dependent through factors $B / n$ and $\left(a_{i} / x_{n}\right)^{2}$, where $a_{i}$ is the local thermal ion gyroradius and $x_{n}$ is the radial gradient length at the plasma-field interface.

Returning to the case of classical resistivity, the uniformity of electrical conductivity follows from the assumption of uniform electron temperature. Then, it is expeditious to multiply Equation (79) by $\sigma \mu_{0}=1 / D$, where $D$ is the resistive diffusivity. Also note the identity $\sigma / n q=\Omega / B$, where $\Omega$ is the Hall parameter $\Omega=\omega_{c e} / v_{e}$, and $B$ therein is the magnitude of the magnetic field (neglecting the azimuthal field component). Note that the electron gyrofrequency $\omega_{c} e^{\propto B}$, whereas the electron plasma frequency $\omega_{p e} \propto \sqrt{n}$. Also, note that the electron collision frequency is $v_{e} \propto n / T_{e}^{3 / 2}$.

We also invoke a vector identity for the $\nabla \times(\mathbf{V} \times \mathbf{B})$ term and recall that $\nabla \cdot \mathbf{B}=0$. Then, upon multiplying through by $\sigma \mu_{0}$, Equation (79) reads

$$
\begin{aligned}
\nabla \times\left[\mu_{0} \mathbf{J}+\left(\frac{\Omega}{B}\right)\right. & \left.\mu_{0} \rho \mathbf{V} \cdot \nabla \mathbf{V}\right|_{\rfloor} \\
& -\frac{1}{D}(-\mathbf{B} \nabla \cdot \mathbf{V}+\mathbf{B} \cdot \nabla \mathbf{V}-\mathbf{V} \cdot \nabla \mathbf{B})=0
\end{aligned}
$$

We shall preclude radial velocity from the plasma model, so that $V_{r}=0$. The inward-radial velocity associated with resistive dissipation (by the longitudinal return current) of magnetic energy in the azimuthal magnetic field is estimated in Appendix E, and is found to be very small. Then, a divergenceless flow condition is satisfied by uniform axially directed flow of plasma, $V_{z}$, that is assumed to be constant in time. Thus, for the purpose of examining penetration of magnetic fields into the plasma core, the plasma core is here modeled as a straight cylinder moving rigidly downstream with a constant axial velocity. The possibility of rotation is allowed, however, so the inertial term does not vanish, even if the density is uniform.

In this situation, the plasma density still is allowed to have a radial gradient, which it must in any case at the boundary of the core plasma. The plasma continuity equation in a steady state reads $\nabla \cdot(\rho \mathbf{V})=0=\rho \nabla \cdot \mathbf{V}+\mathbf{V} \cdot \nabla \rho$. Because of the assumption about the plasma velocity, and the uniformity of the plasma density along the axial direction, each of the two terms on the right-hand side of the continuity equation vanishes, yet the plasma mass density $\rho$ can have a nonuniform radial profile.

It is important to note that in this case of uniform temperature and classical resistivity, the combination $(\Omega / B) \rho$ depends only on the electron temperature and hence is uniform; that is, $\Omega=\omega_{c e} / v_{e}$ and $v_{e} \sim n / T_{e}^{3 / 2}$. This feature is used in carrying out the details in Equation (80). 
In the term $(-\mathbf{B} \nabla \cdot \mathbf{V}+\mathbf{B} \cdot \nabla \mathbf{V}-\mathbf{V} \cdot \nabla \mathbf{B})$ of Equation (80), those terms that contain both $B_{\theta}$ and $V_{\theta}$ cancel one another because the configuration is axially symmetric. It therefore is expedient to define projections of the velocity and magnetic field vectors in the r,Z-plane. Thus, the projection of the flow velocity vector is $\mathbf{V}_{\text {proj }} \equiv(V \cdot Z) \hat{\mathbf{z}}=\left(0,0, V_{Z}\right)$.

One might believe that we could also assume that $B_{r}=0$; that is, that there should be no radial magnetic field component in a straight cylindrical configuration. However, this is not the case. In the lab frame, one has a steady-state boundary-value situation, in which the nozzle magnetic field generally obeys restrictions in or near the breech region of the nozzle. For example, if the backplate is highly conducting, then the nozzle field lines are frozen into the backplate.
This constraint implies that when the $B_{Z}$ component tries to diffuse into the resistive core plasma, the nozzle field lines must be stretched inwards and thereby necessarily must produce a $B_{r}$-component. This would happen even if the backplate were not a conductor, for the nozzle field lines also are somewhat constrained by the very conductive rings that generate the nozzle field itself. The mathematical relation between axial and radial components of magnetic field follows from $\nabla \cdot \mathbf{B}=0$. It reads $r^{-1} \partial_{r}\left(r B_{r}\right)=-\partial_{z} B_{z}$.

Therefore, we must retain $B_{r}$ in principle, and the magnetic field projection vector then becomes $\mathbf{B}_{\text {proj }}=\left(B_{r}, 0\right.$, $\left.B_{Z}\right)$. Then the term $(-\mathbf{B} \nabla \cdot \mathbf{V}+\mathbf{B} \cdot \nabla \mathbf{V}-\mathbf{V} \cdot \nabla \mathbf{B})$ of Equation (80) becomes

$$
\begin{aligned}
& \left(\mathbf{B}_{\text {proj }} \cdot \nabla \mathbf{V}_{\text {proj }}-\mathbf{V}_{\text {proj }} \cdot \nabla \mathbf{B}_{\text {proj }}+\mathbf{B}_{\boldsymbol{\theta}} \cdot \nabla \mathbf{V}_{\text {proj }}+\mathbf{B}_{\text {proj }} \cdot \nabla \mathbf{V}_{\theta}-\mathbf{V}_{\boldsymbol{\theta}} \cdot \nabla \mathbf{B}_{\text {proj }}-\mathbf{V}_{\text {proj }} \cdot \nabla \mathbf{B}_{\theta}\right) \\
& =\left\{\mathbf{0}-V_{Z} \partial_{Z}\left(\mathbf{B}_{Z}+\mathbf{B}_{r}\right)+\mathbf{0}+\left(B_{r} \partial_{r}+B_{Z} \partial_{Z}\right) \mathbf{V}_{\theta}-\left[\left.\left(\frac{V_{\theta}}{r}\right) \partial_{\theta}\right|_{\rfloor}\left(B_{r} \hat{\mathbf{r}}\right)-V_{Z} \partial_{Z} \mathbf{B}_{\theta}\right\}\right. \\
& =\left\{\hat{\mathbf{r}}\left(-V_{Z} \partial_{Z} B_{r}\right)+\hat{\boldsymbol{\theta}}\left[\left(B_{r} \partial_{r}+B_{Z} \partial_{Z}\right) V_{\theta}-\left(\frac{V_{\theta}}{r}\right) B_{r}-\left.V_{Z} \partial_{Z} B_{\theta}\right|_{]}+\hat{\mathbf{Z}}\left(-V_{Z} \partial_{Z} B_{Z}\right)\right\}\right. \\
& =\left\{\hat{\mathbf{r}}\left(-V_{Z} \partial_{Z} B_{r}\right)+\hat{\boldsymbol{\theta}}\left[B_{r} r \partial_{r}\left(\frac{V_{\theta}}{r}\right)+B_{Z} \partial_{Z} V_{\theta}-\left.V_{Z} \partial_{Z} B_{\theta}\right|_{\rfloor}+\hat{\mathbf{z}}\left(-V_{Z} \partial_{Z} B_{Z}\right)\right\}\right.
\end{aligned}
$$

Here, the obvious unit vectors for the cylindrical coordinate system have been introduced. The relation $\partial_{\theta} \hat{\mathbf{r}}=\hat{\boldsymbol{\theta}}$ was used. Also, it is worth noting that the operator $V_{Z} \partial_{Z}$ would play the role of a time derivative in the moving frame.

The next calculation is to write out the fluid-inertial term in Equation (80). The occurrence of the inertial term is a direct consequence of the Hall term in Ohm's law. In evaluating the inertial term, the following vector identity is used:

$$
\nabla\left(\frac{V^{2}}{2}\right)=\mathbf{V} \cdot \nabla \mathbf{V}+\mathbf{V} \times \nabla \times \mathbf{V}
$$

The gradient term on the left-hand side does not survive the curl operator in Equation (80), when the other factors within that term are uniform. Therefore, effectively,

$$
\mathbf{V} \cdot \nabla \mathbf{V}=-\mathbf{V} \times \mathbf{\omega}
$$

where $\boldsymbol{\sigma}=\nabla \times \mathbf{V}$ is the vorticity. Operating on the inertial term with the curl in Equation (80), recalling that $(\Omega / B) p$ is uniform and using Equation (83), yields

$$
\begin{aligned}
& \nabla \times(\mathbf{V} \cdot \nabla \mathbf{V})=-(\mathbf{V} \nabla \cdot \boldsymbol{\omega}-\boldsymbol{\omega} \nabla \cdot \mathbf{V}+\boldsymbol{\omega} \cdot \nabla \mathbf{V}-\mathbf{V} \cdot \nabla \boldsymbol{\sigma}) \\
& =-\left[\boldsymbol{\omega} \cdot \nabla \mathbf{V}_{\theta}-\left(\frac{V_{\theta}}{r}\right) \partial_{\theta} \boldsymbol{\omega}-V_{Z} \partial_{Z} \boldsymbol{\omega} \mid\right]
\end{aligned}
$$

Now, the cylindrical components of vorticity $\left(\Phi_{r}, \Phi_{\theta}, \Phi_{Z}\right)$ are as follows, when noting $\mathbf{V}=\left(0, V_{\theta}, V_{Z}\right)$, with uniform $V_{Z}$ :

$$
\begin{aligned}
\boldsymbol{\omega}=\left[-\partial_{Z} V_{\theta},-\partial_{r} V_{Z},\left(\frac{1}{r}\right) \partial_{r}\left(r V_{\theta}\right)\right] \\
=\left[-\partial_{Z} V_{\theta}, 0,\left(\frac{1}{r}\right) \partial_{r}\left(r V_{\theta}\right)\right]=\left(\omega_{r}, \omega_{\theta}, \sigma_{Z}\right)
\end{aligned}
$$

Using this expression for the vorticity in the previous equation and working out the details, one finds 


$$
\begin{aligned}
& \nabla \times(\mathbf{V} \cdot \nabla \mathbf{v})=\hat{\mathbf{r}}\left(V_{Z} \partial_{Z} \boldsymbol{\varpi}_{r}\right) \\
& -\hat{\boldsymbol{\theta}}\left[\boldsymbol{\varpi}_{r} r \partial_{r}\left(\frac{V_{\theta}}{r}\right)+\left.\varpi_{Z} \partial_{Z} V_{\theta}\right|_{\rfloor}+\hat{\mathbf{z}}\left(V_{Z} \partial_{Z} \boldsymbol{\varpi}_{Z}\right)\right.
\end{aligned}
$$

This result is entirely analogous to Equation (81), except that before in Equation (81), $B_{\theta} \neq 0$, whereas now in Equations $(85)$ and $(86), \varpi_{\theta}=0$.

Finally, we display the first term of Equation (80), using $\mu_{0} J=\nabla \times \mathbf{B}$. Assuming the usual axial symmetry, and in terms of cylindrical coordinates, one finds

$$
\begin{gathered}
{\left[\nabla \times\left(\mu_{0} \mathbf{J}\right)\right]_{r}=-\partial_{Z}^{2} B_{r}+\partial_{Z} \partial_{r} B_{Z}=-\partial_{Z}^{2} B_{r}} \\
\left.-\partial_{r}\left[\left(\frac{1}{r}\right) \partial_{r}\left(r B_{r}\right)\right]\right\rfloor \\
{\left[\nabla \times\left(\mu_{0} \mathbf{J}\right)\right]_{\theta}=-\left\{\partial_{Z}^{2} B_{\theta}+\partial_{r}\left[\left(\frac{1}{r}\right) \partial_{r}\left(r B_{\theta}\right)\right] \|\right\}} \\
{\left[\nabla \times\left(\mu_{0} \mathbf{J}\right)\right]_{Z}=\left(\frac{1}{r}\right) \partial_{r}\left[r\left(\partial_{Z} B_{r}-\partial_{r} B_{Z}\right)\right]}
\end{gathered}
$$

Equations (81), (86), and (87) are used in Equation (80). To simplify the presentation, long-thin ordering for the nozzlemagnetic field geometry is invoked, namely

$$
\begin{gathered}
B_{r} \ll B_{Z} \\
\partial_{Z} \ll \partial_{r} \\
\frac{B_{r}}{B_{Z}} \sim \frac{\delta_{r}}{\delta_{Z}}
\end{gathered}
$$

Here, $\delta_{r} \ll \delta_{Z}$ and symbolize gradient scale lengths in the $\mathrm{r}$ - and Z-directions, respectively, for the special dependence of the magnetic field. The third of these conditions is required by the restriction that the magnetic field have no divergence. Using Equations (81), (86), and (87), the following three component equations are then obtained from Equation (80):

$$
\begin{aligned}
& -\partial_{r}\left[\left.\left(\frac{1}{r}\right) \partial_{r}\left(r B_{r}\right)\right|_{\rfloor}\right. \\
& +V_{Z} \partial_{Z}\left\{\left\{\left[\left(\frac{\Omega}{B}\right) \mu_{0} \rho \mid \varpi_{r}+\left(\frac{1}{D}\right) B_{r}\right\}=0\right.\right.
\end{aligned}
$$

$$
\begin{aligned}
& \left.-\partial_{r}\left[\left(\frac{1}{r}\right) \partial_{r}\left(r B_{\theta}\right)\right]\right\rfloor \\
& -\left[\left(\frac{\Omega}{B}\right) \mu_{0} \rho \mid\right]\left[\varpi_{r} r \partial_{r}\left(\frac{V_{\theta}}{r}\right)+\left.\Phi_{z} \partial_{z} V_{\theta}\right|_{]}\right. \\
& -\frac{1}{D}\left[B_{r} r \partial_{r}\left(\frac{V_{\theta}}{r}\right)+B_{Z} \partial_{Z} V_{\theta}-\left.V_{Z} \partial_{Z} B_{\theta}\right|_{]}\right. \\
& =0 \\
& -\left(\frac{1}{r}\right) \partial_{r}\left(r \partial_{r} B_{Z}\right) \\
& +V_{Z} \partial_{Z}\left\{\left[\left(\frac{\Omega}{B}\right) \mu_{0} \rho \mid \Phi_{Z}+\left(\frac{1}{D}\right) B_{Z}\right\}=0\right.
\end{aligned}
$$

These equations, which describe penetration of fields into core plasma, are evidently very complicated. For example, the azimuthal component equation for $B_{\theta}$ depends on both $B_{r}$ and $B_{Z}$ as obtained from the other two component equations. Moreover, all three component equations depend on the rotational velocity $V_{\theta}$, as calculated from a simultaneous solution of plasma penetrating into field (why computer simulations such as MACH2, extended to include Hall current and rotation, are needed). Nevertheless, it proves possible to obtain some information regarding penetration of fields into core plasma, including cylindrical geometry, Hall current, return current, and plasma rotation.

The first thing that can be done is to find out what this set of equations reduces to when the Hall current is absent. In that case, there is no longitudinal return current; hence $B_{\theta}=0$. Moreover, without Hall current, there is no mechanism to drive up the fluid rotational velocity of the plasma; hence $V_{\theta}=0$. Then the vorticity also vanishes; thus $\boldsymbol{\omega}=\mathbf{0}$ (see Eq. (85)). Under these restrictions, the above three equations reduce to the following:

$$
\begin{gathered}
\partial_{r}\left[\left.\left(\frac{1}{r}\right) \partial_{r}\left(r B_{r}\right)\right|_{\rfloor}=\left(\frac{V_{Z}}{D}\right) \partial_{Z} B_{r}\right. \\
0=0 \\
\left(\frac{1}{r}\right) \partial_{r}\left(r \partial_{r} B_{Z}\right)=\left(\frac{V_{Z}}{D}\right) \partial_{Z} B_{Z}
\end{gathered}
$$

Clearly, these reduced equations for steady-state disposition of the $\mathrm{r}$ - and Z-components of magnetic field exhibit the character of resistive diffusion of the magnetic field, which was the subject of a previous section based upon the simple 
Ohm's law. When the boundary-layer width is small compared to the radius of core plasma, the relative variation of the $r$-coordinate within the plasma-field mixing layer can be neglected, and the equations reduce to a description of diffusion in planar geometry. The only essential difference from the previous section just referred to is that now Equation (90) with respect to the lab frame, is not the moving frame. Thus, the operator $V_{Z} \partial_{Z}$ plays the role of a time derivative in a frame of reference moving with plasma propellant.

In the lab frame of reference, with ancillary boundary conditions in force at the breech of the nozzle, inward diffusion of the $B_{Z}$ component can occur only with the simultaneous appearance and inward diffusion of a $B_{r}$ component. This must be so whether or not the Hall effect is present. The fundamental requirement that the magnetic field be divergence free, $\nabla \cdot \mathbf{B}=0$, forces the appearance of $B_{r}$ with a nonuniform radial profile. This must happen whenever the term $V_{Z} \partial_{Z} B_{Z}$ is significant. Moreover, although the present discussion has been cast in terms of a straight cylindrical plasma core, its conclusions, with allowance for obvious geometric modifications, have meaning as well for a converging-diverging nozzle-shaped geometry.

In Appendix C, a similarity solution is derived for $B_{Z}$ in Equation (90) above, assuming a thin plasma-field mixing layer. The solution reads

$$
B_{Z}=B_{\mathrm{pe}}\left[1-\operatorname{erf}\left(\frac{X}{2 \sqrt{u_{\tau}}}\right)\right]
$$

where $B_{\mathrm{pe}}$ is the assumed-uniform value of $B_{Z}$ at the plasma edge, $X$ is the inward radial distance from the plasma edge, and $u_{\tau}=Z D / V_{Z}$ is a time-like similarity variable. In a more realistic scenario, the edge field would be nonuniform along $Z$, since the starting flux between the plasma and the wall would be preserved because that flux spread out downstream during the inward diffusion of the magnetic field. However, such a scenario is not accessible to a similarity solution. We ask for the value of $X$ where the field is reduced to, say, onethird of its edge value. For the value of $\tau$, a characteristic length $Z=1 \mathrm{~m}, D=3 \mathrm{~m}^{2} / \mathrm{s}$, and $V_{z}=10^{5} \mathrm{~m} / \mathrm{s}$ are input, in accordance with Table I for parameters of hydrogen propellant. The value of $X$ is then chosen so that the value of the error function is $2 / 3$. The result is that the characteristic length of protrusion of magnetic field into the plasmaconsidered as a simple resistive medium -is $X=0.65 \mathrm{~cm}$, in excellent agreement with the earlier calculation based upon a different approach.

We return now to the opposite case, where the Hall current, its return current, and plasma rotation are present. A complete discussion is beyond the purview of this report. It is sufficient here to just focus upon Equation (89c), which describes the steady-state disposition of the longitudinal magnetic field relative to core plasma. For convenience, that equation is reproduced here:

$$
\begin{aligned}
& -\left(\frac{1}{r}\right) \partial_{r}\left(r \partial_{r} B_{Z}\right) \\
& \quad+V_{Z} \partial_{Z}\left\{\left\{\left[\left(\frac{\Omega}{B}\right) \mu_{0} \rho \mid\right]^{\top} \sigma_{Z}+\left(\frac{1}{D}\right) B_{Z}\right\}\right)=0
\end{aligned}
$$

Let us compare the first term with the second term, the Hall term. Equation (62) is used in connection with the vorticity $\boldsymbol{\sigma}_{z}$. That equation states that the ion rotational current density is given by the ion magnetization current density; that is, $n q V_{\theta} \approx B^{-1} \partial_{r} P_{i}$. It is not significantly material whether $B$ is regarded as $B_{Z}$ or as the total magnetic field including $B_{\theta}$. Then one can easily estimate, to order of magnitude, the ratio of the second term of Equation (89c) to the first term. The ratio proves to be $\beta_{i}\left(\lambda_{e} / L_{\varpi}\right) \sqrt{m_{i} / m_{e}}$. Here, $V_{Z} \partial_{Z} \sim V_{\text {th } i} / L_{\varpi}$, where $V_{\text {th } i}$ is a representative ion thermal velocity and $L_{\bar{\varpi}}$ is a characteristic axial length related to the development of vorticity in the plasma-field mixing layer. For $\beta_{i}$ on the order of 1 , when the quantity $L_{\varpi} / \lambda_{e} \sqrt{m_{e} / m_{i}}$ exceeds 1 , the Hall term is unimportant and consequently ordinary resistive diffusion of field into plasma is obtained. This condition essentially agrees with that needed for resistive diffusion of plasma into field in the presence of Hall current and rotation, as described in connection with Equations (74) to (76) (see $L_{\text {crit }}$ discussion in Section 2.3.2.2, "Hall current allowed (voltage shorted out)". Therefore,

$$
L_{\varpi} \approx \lambda_{e} \sqrt{\frac{m_{i}}{m_{e}}} \approx L_{\text {crit }}
$$

In the opposite extreme, if the Hall term were very large, one might expect that the radial gradients of $B_{Z}$ would have to become very large in the first term of Equation (89c) to keep that equation in balance. The "sharp boundary" picture emerging from such a scenario implies that $B_{Z}$ has not diffused very much into the core plasma.

It is instructive to take a somewhat different approach to the issue of resistive diffusion of field into plasma as regards the alteration of this process by the Hall effect and rotation. Note that

$$
\left(\frac{\Omega \mu_{0} \rho}{B}\right)=\left(\frac{m_{i}}{q D}\right)
$$


Here, as usual, $m_{i}$ is the mass of an ion, $q$ is the magnitude of the electron's charge, and $D$ is the resistive diffusivity. In this section, we are assuming singly charged ions. Then Equation $(89 \mathrm{c})$ can be rewritten as

$$
\left(\frac{1}{r}\right) \partial_{r}\left(r \partial_{r} B_{Z}\right)=\left(\frac{V_{Z}}{D}\right) \partial_{Z}\left(B_{Z}+\frac{m_{i} \Phi_{Z}}{q}\right)
$$

Recall Equation (85), in particular that the longitudinal component of vorticity $\bar{\sigma}_{Z}$ is related to the ion-fluid rotational velocity by $\Phi_{Z}=r^{-1} \partial_{r}\left(r V_{\theta}\right)$. Utilizing again the ion magnetization current density $B^{-1} \partial_{r} P_{i}$ to represent the ion-fluid azimuthal current density $n q_{i} V_{\theta}$, it can then be qualitatively estimated that the vorticity term $\left(m_{i} / q_{i}\right) \varpi_{Z}$ in Equation (94), can become significant in comparison to the $B_{Z}$ term when the plasma-field mixing layer width (for diffusion of plasma into field) is small on the order of an ion gyroradius, $a_{i}$.

A more quantitative estimate proceeds as follows. Factoring out $m_{i} / q_{i}$, one has in Equation (94) a comparison of the ion gyrofrequency, $\omega_{c i}$ with the vorticity component $\boldsymbol{\sigma}_{Z}$. Then for a radially uniform ion temperature, a radially uniform magnetic field, and invoking the magnetization current density, one can then estimate the vorticity component for a given density profile. The simplest case is a linearly decaying density profile of the form $n(x)=n_{\text {core }}[1-x / \delta]$, where $x$ is the radial distance outwards from the nominal plasma edge and $n_{\text {core }}$ is the number density in the core propellant. Then one finds

$$
\begin{aligned}
\bar{\omega}_{Z} & \approx \partial_{x}\left(\frac{n q V_{\theta}}{n q}\right)=\partial_{x}\left[\left.\left(\frac{1}{n q B}\right) \partial_{x} P_{i}\right|_{\rfloor}\right. \\
& =\left(\frac{T_{i}}{q B}\right) \partial_{x}\left[\left.\left(\frac{1}{n}\right) \partial_{x} n\right|_{\rfloor}=-\frac{1}{2}\left(\frac{a_{i}}{\delta}\right)^{2}\left(\frac{n_{\text {core }}}{n}\right)^{2} \omega_{c i}\right.
\end{aligned}
$$

Thus, the vorticity $\boldsymbol{\sigma}_{Z}$ is comparable to or larger than the ion gyrofrequency $\omega_{c i}$ when the plasma-into-field layer width $\delta$ is small on the order of the ion gyroradius $a_{i}$ and particularly in the low-density edge of the layer. This admittedly non-self-consistent argument indicates that when the plasmainto-field layer width $\delta$ is on the order of $a_{i}$, then also under the same condition, a large Hall effect manifested as ionfluid rotation acts to dominate resistive penetration of field into plasma.

Assuming that the Hall-vorticity term does indeed dominate the right-hand side of Equation (94), one can then proceed to estimate the field-into-plasma penetration depth $\delta_{B}$ as follows. According to the remarks in the previous paragraph $\left|\omega_{Z}\right| \approx\left|C_{\mathrm{I}}\right| \omega_{c i}$, where $\left|C_{\mathrm{I}}\right|$ is a number exceeding 1 (see
Eq. (95)). Then, equating the left side of Equation (94) to the Hall term on the right side one has the following:

$$
\begin{gathered}
\frac{1}{\delta_{B}^{2}}=\frac{C_{I} V_{t h i}}{D L_{\varpi}} \\
\delta_{B}^{2} \approx \frac{L_{\varpi} D}{C_{\mathrm{I}} V_{\text {th } i}}=\frac{1}{C_{\mathrm{I}}}\left(\frac{c}{\omega_{p e}}\right)^{2} \frac{v_{e} L_{\varpi}}{V_{\text {th } i}} \\
=\frac{m_{e}}{C_{\mathrm{I}} m_{i}}\left(\frac{c}{\omega_{p i}}\right)^{2} \frac{v_{e} V_{\text {th } e} L_{\varpi}}{V_{\text {the } e} V_{\text {th } i}}
\end{gathered}
$$

Here, the expression $\left(1 / \sigma \mu_{0}\right)$ is inserted in place of $D$ and set in terms of the electron plasma frequency, $\omega_{p e}=\sqrt{n q^{2} / \varepsilon_{0} m_{e}}$. Also, the corresponding ion plasma frequency $\omega_{p i}$ is introduced. Then the relation $\varepsilon_{0} \mu_{0}=1 / c^{2}$ is invoked, where $c$ is the speed of light in mks units. Finally, the electron thermal velocity $V_{\text {th }} e$ is also introduced. For simplicity, electron and ion temperatures are assumed equal.

Recalling that the electron collision mean free path is given by $\lambda_{e}=V_{\text {th }} e_{e}$, the above equation for the square of the field-penetration depth becomes the following:

$$
\delta_{B}^{2} \approx \frac{1}{C_{\mathrm{I}}} \sqrt{\frac{m_{e}}{m_{i}}}\left(\frac{c}{\omega_{p i}}\right)^{2} \frac{L_{\varpi}}{\lambda_{e}}
$$

In the present example of a dominant Hall effect, the product $\left(L_{\varpi} / \lambda_{e}\right) \sqrt{m_{e} / m_{i}}$ is smaller than unity, in contrast to the situation with resistive diffusion. Hence, the field protrusion depth is $\delta_{B}<\left(c / \omega_{p}\right)$, which is known as the "ion inertia length." Since $a_{i}=\left(c / \omega_{p}\right) \sqrt{\beta_{i}}$, the ion inertia length is on the order of the ion gyroradius whenever $\sqrt{\beta_{i}}$ is on the order of 1 .

We conclude that the field protrusion depth into the plasma is on the order of the ion inertia length (the ion gyroradius), provided that the characteristic axial length does not exceed a certain critical value. This is essentially the same critical length found for the Hall effect to dominate resistive diffusion of plasma into field. It is important to emphasize that the Hall effect and plasma rotation had to be included together in order to reach Equation (96c).

\subsection{Summary}

Since Section 2.0 is lengthy, we pause here for an interim summary and discussion of its contents and a few recommendations for future work. 
First, a simple model of quasi-one-dimensional nozzle flow was presented based upon the Bernoulli equation. This well-known model affords useful estimates of propellant flow speeds and other quantities as related to the injected propellant properties; for example, the propellant speed was derived at the nozzle throat and at the exit plane.

Then, we indicated how the longitudinal flow becomes distorted by the presence of curved magnetic field lines and edge pressure gradients when the plasma propellant is not a perfect conductor. Using the simplest form of Ohm's law, we displayed the eddy current braking of propellant flow across flux surfaces. For the tabulated parameters of interest, which include an assumption of classical resistivity, we showed that eddy current braking in the region upstream of the nozzle's throat severely limits the growth of the resistive boundary layer of edge plasma, as generated by pressuregradient-driven plasma diffusion into the confining magnetic nozzle field. The growth of this plasma-field mixing layer is relevant to the amount of attached plasma that evolves during transit of the nozzle. A more detailed estimate of the layer thickness is presented at the end of this summary section.

The implicit assumption in our treatment was that, within the resistive MHD model, there has occurred no sudden initial penetration of magnetic field and plasma. Such behavior might be caused by dynamical resistive MHD effects induced by rapid spatial convergence of field lines downstream as induced by upstream plasma impact. Early lateral displacement of field lines by the highly conducting injected plasma, together with the unavoidable crowding of flux into the nozzle's throat, could enhance the spatial rate of convergence of flux beyond the breech of the nozzle, instigating strong curvature and thereby spoiling the gentle convergence of propellant flow. Coil discreteness can exacerbate that effect. Dynamical resistive MHD processes that may be engendered by such rapid spatial changes need to be understood and avoided, if possible. References 1 to 3 have identified such behavior in an axisymmetric simulation and has pointed out its importance. A non-MHD effect that causes instant penetration of field and injected plasma is demonstrated in Section 3.0, "Resistivity From GradientDriven Microinstabilities." In other words, the injection process is here assumed to be sufficiently smooth and gradual as to be benign. A systematic investigation of the effects of coil discreteness and the rapidity of spatial convergence, within the axially symmetric resistive MHD model, would be a worthwhile and instructive project.

Although the propellant temperature drops precipitously after passage through the throat of the nozzle (see Ref. 6), it hardly drops at all between breech and throat. Therefore, classical resistivity should still be small up to the throat. This leads to the expectation that for $1 \mathrm{~m}$ or less of travel (from breech to throat), there should be very little growth of the resistive plasma-field mixing layer separating core-plasma from magnetic nozzle field, in the absence of anomalous resistivity, when the simple Ohm's law applies.
A separate companion process, which also generates attached plasma, is the diffusion of external magnetic field into the plasma - considered as a fixed resistive medium. This process also was analyzed on the basis of the simple form of Ohm's law with classical resistivity and was found to produce essentially the same width of plasma-field mixing layer as previously estimated for the other process. This result was demonstrated by two independent methods, which agreed very well. The first was from the point of view of the moving propellant, with an assumed growing timedependent field applied at the boundary. The second was from the lab frame point of view, assuming a steady-state configuration with a constant external magnetic field.

It is convenient to encapsulate these results in terms of a magnetic Reynolds number, defined as $\operatorname{Re}_{\text {mag }}=V_{A} r / D_{c l}$. Here, the Alfven speed is $V_{A}=\sqrt{B^{2} / \mu_{0} \rho}$, with $B$ being the magnitude of the nozzle magnetic field, and $\rho$ the propellant mass density. Also, $r$ is a representative radius of the confined plasma in the nozzle, and $D_{\mathrm{cl}}=\eta_{\mathrm{cl}} / \mu_{0}$ is a representative resistive diffusivity of plasma propellant. For the purposes of making approximate estimates, the magnetic Reynolds number may be evaluated at the throat of the nozzle. When $\sqrt{\beta} \approx 1$, that is, $V \approx V_{\text {th } i} \approx V_{A}$, with $V_{\text {th } i}$ being the ion thermal speed, the normalized width $\delta$ of the plasmafield mixing layer may be characterized as

$$
\frac{\delta}{r} \sim \frac{1}{\sqrt{\mathrm{Re}_{\mathrm{mag}}}} \sqrt{\frac{L_{b t}}{r}}
$$

where $L_{b t}$ is a characteristic nozzle axial length. As a numerical example, in the region just upstream of the throat $\operatorname{Re}_{\text {mag }} \approx 10^{4}$, and $L_{b t} / r \approx 10$ for $L_{b t} \approx 1 \mathrm{~m}$ and $r \approx 0.1 \mathrm{~m}$. Then $\delta / r \sim 0.03$. This ratio will grow further downstream. The analytical description of the entire evolution of $\delta$ over the length of the nozzle is beyond the purview of this report. Equation (97) thus is relevant to the plasma attachment process.

Turchi (Ref. 8, private communication) points out that if the angle $\phi_{\mathbf{B} \mathbf{V}}$ between field and flow were regarded as an independent parameter, and if the transverse pressure gradient were neglected, then the boundary-layer width would scale rather differently, namely as

$$
\frac{\delta}{r} \sim\left(\frac{1}{\operatorname{Re}_{\mathrm{mag}}}\right) \sin \phi_{\mathbf{B V}}
$$

This result can be derived from the inertial component of the plasma-momentum equation across the flux surfaces (the $x$-component) while incorporating the simple form of Ohm's law for $\mathbf{J}$ within the $\mathbf{J} \times \mathbf{B}$ force. In that manner, one finds that the incident "core" velocity component across the flux 
surfaces, $V_{x \text { core }}=V \sin \phi_{\mathbf{B V}}$, experiences a slowing down due to eddy current braking, with a characteristic time $t_{x \text { char }}=D / V_{A}^{2}$. The boundary-layer width then can be estimated as $\delta \sim V t_{x \operatorname{char}} \sin \phi_{\mathbf{B} \mathrm{V}}$. Assuming that $\sqrt{\beta} \approx 1-$ that is, $V \approx V_{\text {th } i} \approx V_{A}$-one immediately arrives at the expression in Equation (98). The latter constitutes a much stronger dependence on $\mathrm{Re}_{\text {mag }}$.

The extremely short slowing-down time for inertially driven cross-flux motion, which is the result of eddy current braking for the upstream parameters of interest, suggests that the angle between field and flow may not be independently specified in a highly conducting plasma. Section 4.0, "Rayleigh-Taylor- (RT-) Type Instabilities" quantitatively estimates the crossfield braking time within the single-fluid resistive MHD model and the consequent shape of the propellant streamlines. The flow would very quickly become practically field aligned, with a very small remnant of crossfield flow driven by the edge pressure gradient. Therefore, it would appear that the model that underlies Equation (98) is more likely to be valid in the low-pressure high-resistivity region that exists downstream of the throat, where detachment is of interest rather than attachment. There, the magnetic Reynolds number is much smaller, so that the flow angle (relative to the magnetic field) would be less vulnerable to alteration by eddy current braking and also would be less influenced by the broader transverse pressure gradients. The latter point of view was, in fact, adopted for the detachment calculation in Reference 7 . It is therefore recommended that the scaling of the boundary-layer thickness be studied as a function of position along the nozzle in order to elucidate the transition between Equations (97) and (98).

The small amount of plasma-field intermixing estimated upstream of the throat, for the parameters of interest and using classical resistivity, serves as a prompt to consider possible causes why simulations or experiments might nevertheless exhibit substantially greater boundary-layer widths at the nozzle's throat. Such causes could be as follows:

(1) numerical diffusion

(2) degree of processes allowed (Hall effect, rotation, ion viscosity, classical vs. anomalous resistivity)

(3) global geometrical configurations (number and placement of discrete field coils, types of global electrical boundary conditions including plasma effects)

(4) a subtle combination thereof

A feature of the boundary-layer physics notably absent from this report, so far, is ion viscosity. This feature is introduced later in the discussion of Rayleigh-Taylor type instabilities in Section 4.3, "Finite Larmor Radius (FLR) Stabilization of RT-Type Flute Modes." It can be shown that the collisionless contribution to the force term arising from ion gyroviscosity (see Ref. 9) bears essentially the same order-of-magnitude relation to ion inertia in the plasma-momentum equation as does the Hall term to the motional electric field in Ohm's law; that ratio is on the order of ion gyroradius to the plasma's radial scale length. Therefore, collisionless ion gyroviscosity ideally should be included in the plasma model of the interface whenever Hall effects are included.

In this concluding discussion of the general physics topic, we turn now to a review of the underlying physics represented in the modeling of the edge plasma during plasma flow through magnetic nozzles, specifically, the physics associated with the complete Ohm's law (the electron momentum equation). The derivation of the general Ohm's law was first outlined, and then several special cases were examined. The emphasis was on the Hall effect and plasma rotation. A major concern, however, was the justification for use of the simple Ohm's law while in the presence of a strong magnetic field. The simple Ohm's law is often perceived as being the limit of the complete Ohm's law when the electron collision frequency is very large, $v_{e} \gg \omega_{c}$.

The principal results were as follows: First, it was pointed out that when the Hall current is completely suppressed by electrically insulating global boundary conditions, the simple form of Ohm's law is restored by means of the quasi-radial Hall voltage. This result is obtained even though, in the case of interest, $v_{e} \ll \omega_{c e}$. This justifies the resistive diffusion result obtained with the simple Ohm's law, which governs the spatial growth of the plasma-field mixing layer. Practically the same result for the plasma-field mixing layer growth was obtained from two distinct points of view, plasma diffusion into magnetic field and field diffusion into plasma.

Next, in simulations that may short out the Hall voltage and thereby allow Hall current to flow - but inconsistently without plasma rotation - it was shown that there would be a very large reduction in the effective electrical conductivity of plasma. This is the phenomenon of "magnetoresistance," which would enhance edge-plasma diffusion across the flux surfaces. Magnetoresistance associated with the Hall effect has been observed in gas-plasma and solid-state-plasma experiments configured so that rotation is impossible.

Finally, self-consistently allowing for flow of Hall current and plasma rotation, a characteristic length $L_{\bar{\sigma}}$ along the nozzle was identified beyond which the above-mentioned resistive spatial growth of the plasma-field mixing layer would occur. However, for lengths smaller than $L_{\bar{\sigma}}$, the plasma-field mixing layer width proved to be insensitive to the electron collision frequency; instead, it was on the order of the ion gyroradius. Again, essentially the same results were obtained from the two points of view: penetration of plasma into field and penetration of field into plasma. The characteristic length $L_{\varpi}\left(\approx L_{\text {crit }}\right)$ was identified approximately as $L_{\bar{\sigma}} \approx \lambda_{e} \sqrt{m_{i} / m_{e}}$, where $\lambda_{e}$ is the collision mean free path of a representative electron, $m_{i}$ is the mass of an ion, and $m_{e}$ is the mass of an electron. Because the insensitivity of layer 
thickness to electron collisionality is a favorable result, it is recommended that Hall-MHD simulations be performed in which the Hall space-charge electric field is shorted out and rotation of plasma is allowed, while systematically varying the length of the nozzle configuration. Beyond the length $L_{\bar{\omega}}$, plasma spinup produces a quasi-radial motional electrical field that suppresses the flow of Hall current. We then recover the preceding results where the Hall current was not allowed to flow.

Lastly, in this concluding discussion of Section 2.0, on general physics of the magnetic nozzle, we turn to the topic of the dynamical dwell time available for resistive growth of the plasma-field mixing layer. The model that was employed earlier in this report simply assumed that the dwell time is on the order of $L_{b} / V_{t}$, where $L_{b t}$ is a characteristic axial length of the nozzle and $V_{t}$ is the velocity of propellant in the throat. Now, we shall refine that model by recognizing that the dwell time (say, from breech to throat) must be longer because of the time required for acceleration of the propellant up to speed. The plasma-field mixing layer thickness at the throat will be calculated using this refined model.

From Equation (19) with $\beta \approx 1$ in the transition layer, the algorithm that is used to investigate the effect of increased dwell time between breech and throat is

$$
\delta^{2}=\int_{0}^{t_{b t}} D_{\mathrm{cl}} \mathrm{d} t
$$

where $\delta$ is the thickness of the plasma-field mixing layer, $t_{b t}$ is the time required for the propellant to traverse the distance from breech to throat, and $D_{\mathrm{cl}}$ is the classical (Spitzer) resistive diffusivity. It is known that $D_{\mathrm{cl}}$ is insensitive to plasma density and essentially depends only on electron temperature. Equation (99) is analyzed in Appendix B, using a generic shape for the nozzle. There, the time variable is related to the flow velocity, the longitudinal distance along the nozzle $L_{b t}$, and the nozzle's geometry (i.e., the contraction ratio). The nominal plasma edge layer width at the throat $\sqrt{D_{t \mathrm{cl}} L_{b t} / V_{t}}$ is found to be enhanced by the longer dwell time, but by less than a factor of about 2 from the original estimate. Specifically, for a radius-contraction ratio of 2 the bounding numerical factor proved to be 1.7 , whereas for a radius-contraction ratio of 3 it proved to be 2.3 .

From these results, an approximate overestimate of the fraction of attached hydrogen plasma propellant at the throat can now be made for the sample parameters, with the assumptions of classical resistivity and no Hall current. First, the $0.6-\mathrm{cm}$ estimate of $\delta$ is doubled to allow for bidirectional diffusion (plasma into field and field into plasma). Then we multiply by another factor of 2 to allow for the effect on the layer thickness of the increase in dwell time from its reference value $\sqrt{D_{t \mathrm{cl}} L_{b t} / V_{t}}$. This adjustment is for the time required during acceleration of propellant from breech to throat, over a length of $1 \mathrm{~m}$. The final result is a plasma-field mixing layer at the throat of about $2.5 \mathrm{~cm}$. Taking a nozzle's nominal plasma radius at the throat, $r=10 \mathrm{~cm}$, this leads to an upper bound of the attached fraction $2 \delta / r$ of about 50 percent. The factor 2 comes from the geometrically enlarged area of an outer annulus of thickness $\delta$ in cylindrical geometry. To the extent that this result is even approximately realistic, it suggests a search for mitigating effects. Therefore, an example of operation at higher stagnation temperature and lower resistivity with a heavier propellant ion, to achieve the same specific impulse, could be pursued at a future time.

Since the result for the layer thickness is insensitive to the radius-contraction ratio, essentially three options for remediation are allowed, assuming classical resistivity: The first, suggested by Turchi, is to shorten the length $L_{b t}$ from breech to throat. However, the thickness of the plasma-field mixing layer only scales as $\sqrt{L_{b t}}$, assuming that the scaling in Equation (97) is valid. The second is to enlarge the (plasma) throat, which has the disadvantage of increasing the massflow rate of propellant (for fixed stagnation density and stagnation temperature), with consequences for vehicle design and mission logistics. Hence, the second option would entail a smaller propellant density to preserve the mass flow rate $\dot{m}$. The consequent enlargement of the ionion collision mean free path may require extending the MHD flow model into the kinetic regime. The third option is to work at a higher stagnation temperature in order to decrease the resistive diffusivity $D$ (to increase the magnetic Reynolds number) and also to work with heavier propellant atoms to maintain the desired exhaust velocity (specific impulse). See the discussion on constraints at the end of this discussion section.

The above estimate of plasma-field mixing layer thickness, applied to hydrogen propellant with $L_{b t} \approx 1 \mathrm{~m}$ from breech to throat, can as well be applied to the envisioned "Godzilla" experiment (see Sec. 2.1, "Bernoulli Equation for Nozzle Flow") with fully stripped helium propellant and $L_{b t} \approx 0.3 \mathrm{~m}$ (see Ref. 2). According to the tabulated parameters, taking into account the higher stagnation temperature in helium and that the atomic number $\Xi$ is $2, D$ is about $2 / 3$ of the hydrogen value; hence, $\sqrt{D}$ is about 0.8 of the hydrogen value. Also $L_{b} t$ is $1 / 3$ of the previous value, so $\sqrt{L_{b t}}$ is about 0.6 of the previous value. The velocity near the throat, $V_{t}$, is about the same for both propellants. Consequently, the previous answer for the boundary-layer width $\delta$, namely $\sqrt{D_{t \mathrm{cl}} L_{b t} / V_{t}}$, is to be multiplied by $0.6 \times 0.8 \approx 0.5$. Since the previous $\delta$ for hydrogen propellant was about $2.5 \mathrm{~cm}$, the new estimate for helium now is $\delta \approx 1.3 \mathrm{~cm}$.

Figure 6 of Reference 2 displays results relevant to the planned helium experiment, as obtained by the MACH2 
simulation using classical resistivity. It indicates that, at the throat, the radius of core plasma is only about $2 \mathrm{~cm}$. From there, the magnetic field drops off from its nominal edge value of 0.75 to $0.28 \mathrm{~T}$ on axis over only a radial distance of about $2 \mathrm{~cm}$. This drop-off ratio is close to a factor $1 / e$; hence, $2 \mathrm{~cm}$ would appear to constitute a fair representation of the characteristic boundary-layer width obtained by $\mathrm{MACH} 2$ at the throat (compared with the above overestimate of $1.3 \mathrm{~cm}$ ). The plasma density or pressure profiles (not shown in the figure) might have indicated some additional outward penetration of plasma into field, leading to a somewhat thicker effective plasma-field mixing layer. Since both field penetration into plasma and plasma penetration into field engender plasma attachment, it is recommended that the type of information in the figure henceforth be made more complete by providing the radial density and pressure profiles of plasma corresponding to the magnetic profiles therein. The He parameters and coil configuration employed in Reference 2 leading to a 2-cm plasma throat signify that essentially all of the He propellant has become attached to magnetic flux at the throat position.

Because the plasma's corresponding density profile or pressure profile was absent from Figure 6 in Reference 2, our assessment of what transpired in that simulation between breech and throat is necessarily limited. What can be said is that the model of classical resistive diffusion developed in Section 2.0 of this report is in fair agreement with the MACH2 simulation of Reference 2, being too small by perhaps a factor of about 2 or 3 . This is in spite of the fact that the derivation carried out in Appendix B was biased in several respects towards too much interdiffusion.

Figure 8 of Reference 3 shows radial profiles in the plasmanozzle throat for a larger system. The system length is $1 \mathrm{~m}$, and the system radius is $18 \mathrm{~cm}$. The radial plasma pressure profile is shown, along with the radial magnetic pressure profile. At the throat of the nozzle-shaped plasma, the interface width extends from about 3 to about $7 \mathrm{~cm}$; thus, the width is about $4 \mathrm{~cm}$. For comparison, a rough estimate of the resistively diffused width can be obtained from the expression $\sqrt{D t}$. For helium at $100 \mathrm{eV}, D \approx 2.3 \mathrm{~m}^{2} / \mathrm{s}$. Also, $t \approx 10 \mu \mathrm{s}$, from a quasi-one-dimensional isentropic flow model (velocity in the throat $\approx 10^{5} \mathrm{~m} / \mathrm{s}$ ). Then, the resistively diffused width proves to be about $0.5 \mathrm{~cm}$, which is an order of magnitude smaller than the width observed in the MHD simulation. (A factor of 2 or 3 enhancement due to the dwell time during speed-up does not resolve this discrepancy. See Appendix B.) An anomalous process within the resistive MHD model ultimately is held responsible for the 50 percent mass loss, which is tentatively ascribed to the discrete magnetic geometry. In the present report, no attempt is made to address this anomalous process, which appears to lack a complete and definitive understanding. The authors of Reference 3 and this author are in agreement that the observed anomalous mass loss can probably be mitigated by employing a more gradual and smoother magnetic geometry transition between the nozzle inlet and the plasma-nozzle throat. Such an adjustment may require elongated inlet geometry. This speculation ought to be tested with more numerical MHD simulations.

The influence of the nozzle shape of core plasma on the accrued diffusive edge-layer thickness yet remains to be thoroughly investigated. Measured magnetic nozzle fieldline shapes in a real experiment, albeit in coaxial geometry over part of the effective length of the thruster, are displayed in Figure 1 of Reference 11. Therein, resistive drag by the plasma substantially modifies the field-line shape.

Although the occurrence of substantial diffusive plasma attachment motivates exploration of improvements in design, it should be recognized that there are fundamental constraints arising out of mission logistics. For example, the two quantities $\mathrm{d} m / \mathrm{d} t=\dot{m}$ and the specific impulse $V_{e} / g$ ought to remain fixed for a given mission when searching for improved parameters. The latter constraint implies that the stagnation temperature is fixed for a given propellant; the former constraint implies that the quantity $\rho r^{2}$ is fixed (say at the throat). Here, $\rho$ is the mass density of propellant and $r$ is the throat radius of the confined plasma core.

However, there is a third constraint associated with the magnetic nozzle: the external magnetic pressure should balance the internal plasma pressure transversely. The consequent condition on the global beta, namely that $\beta \approx 1$, along with fixed stagnation temperature, implies that $\rho$ scales as $B^{2}$. Therefore, the product $B r$ must be fixed at the throat. A fixed temperature then implies that the ratio $r / a_{i}$ is fixed; that is, that the ratio of ion gyroradius $a_{i}$ to the effective plasma-throat radius $r$ is fixed-for a given propellant. This result was shown in Reference 2. In terms of different propellants, the ratio $a_{i} / r$ scales approximately as $m_{i} /(\Xi)^{3 / 2}$ for fixed $\dot{m}$ and specific impulse, where $m_{i}$ is the ion mass and $(\Xi)\left(1.6 \times 10^{-19} \mathrm{C}\right)=q_{i}$ is the ion charge. In this report, $\left|q_{i}\right|$ $=\left|q_{e}\right|$, and only hydrogen propellant is of interest.

This constraint on $a_{i} / r$ should be remembered when contemplating the use of Hall current and rotation along with axial lengths shorter than $\lambda_{e} \sqrt{m_{i} / m_{e}}$ in order to limit the plasma-field mixing layer thickness to the ion gyroradius. This constraint also is important when considering the activity of certain microinstabilities at the plasma-field interface, such as the LHD instability. The linear and nonlinear properties of the latter depend fundamentally on the size of the ion gyroradius relative to transverse plasma gradient lengths such as the thickness of the plasma-field interface.

Finally, it should be emphasized that in this report the concept of axially symmetric resistive MHD flow with a relatively small rate of resistive interdiffusion at the plasmafield interface has been taken for granted. If ill-understood resistive MHD dynamical mixing processes were to exist in the breech because of the severity of the plasma injection process, and perhaps as exacerbated by the discreteness 
of field coils, those processes would certainly have to be identified and eliminated. Otherwise, supposedly educated attempts at systematic engineering designs of magnetic nozzles would be premature.

\subsection{Resistivity From Gradient-Driven Microinstabilities}

In this section, we review the research that has been done on certain microinstabilities, which can potentially contribute to anomalously rapid broadening of the plasma-field interface. Attention is focused primarily on those modes that propagate in the azimuthal direction, directly across the magnetic field.

Plasma density gradients that have been formed across a confining magnetic field are often regarded, within the context of perfect symmetry, as equilibrium states of ideal dynamical force-balance. In a practical sense, however, they constitute nonequilibrium configurations precariously suspended away from states of lower energy. That is because perfect symmetry is never attained. Therefore, such configurations are actually vulnerable to symmetry-destroying plasma instabilities that grow out of slight asymmetries and that try to access the lower states. For the magnetic nozzle, the macroscopic consequence would be a loss of definition of the plasma-field interface, with thickening of the plasma-field mixing layer, and concomitant attachment of peripheral propellant to magnetic nozzle flux. In fact, even a macroscopically smooth resistive fluid model entails a graininess and concomitant loss of symmetry at the microscopic level, leading to resistive diffusion.

Some of these instabilities have very large growth rates and are not amenable to descriptions within a resistive magnetofluid MHD model. The LHD instability is one that has been identified as a major concern in such situations. It is nominally a robust nonresonant instability that does not require the extreme conditions needed by some other wellknown microinstabilities. For example, it does not need current drift velocities in excess of the electron thermal speed as would be required by the Buneman instability. It is insensitive to the electron-ion temperature ratio and so does not need $T_{e} \gg T_{i}$ as would be required for destabilization of the ion-acoustic instability. Reference 12 presents simulations of screw-pinch and theta-pinch magnetically driven implosions using a hybrid computer simulation (particle ions and fluid electrons), which employed the so-called Chodura resistivity. This is an empirically parametrized representation of electron resistivity arising from the ion-acoustic instability. However, they ignored the instability requirement that $T_{e} \gg T_{i}$. In their model, the current drift velocity correctly is required to exceed the ion-acoustic speed, which is a much more stringent requirement than in the LHD instability. Then, in the empirical model, the electron collision frequency is scaled by the ion plasma frequency.
(Chodura resistivity is an option in the MACH2 resistiveMHD computer simulations.) Sgro and Nielson had some limited success with this approach. They computed the structure of the magnetic field in magnetically imploded plasmas and generated reflected ions in front of the magnetic piston; nevertheless, the empirical resistivity lacked a firm theoretical foundation, and there was difficulty in treating the late phases of those implosions. Regarding the validity of the empirical resistivity, it should also be remarked that Chodura himself had a similar degree of success in simulations of magnetically compressed plasma, but employed a rather different electron collision frequency: namely, the constant 0.025 multiplied by the electron gyrofrequency in the external magnetic field (see Fig. 1 in Ref. 13). Also, the LHD instability can be destabilized by weak plasma gradients, having gradient lengths that are large compared to the ion gyroradius. Moreover, although nominally stabilized by finite $\beta$, it persists into the ion-cyclotron regime even where the local $\beta$ is substantially larger than 1 . (For a brief review of the various properties of this instability, see the introductory discussion in Ref. 14 and the other references therein.)

In the magnetic nozzle, the linear phase of the LHD instability would manifest itself as a fine-scale rotating azimuthal ripple (flute-type mode) in the plasma-field interface region. In this report, the linear and nonlinear theories of this instability will be briefly reviewed. The nonlinear phase evolves into a form of plasma turbulence that would be macroscopically evident as an enhanced resistivity. Whether such enhancement dominates over classical resistivity depends upon parameters such as the electron temperature and the local width of the plasma-field mixing layer.

In this report, the nonlinearly evolved microturbulent LHD resistivity will be compared with the classical resistivity that arises from coulomb collisions of electrons on ions. Although the influence of finite $\beta$ on the linear phase of the instability has been clarified (Ref. 15), this writer believes that the $\beta$ dependence of the evolved microturbulence (Ref. 14) is not yet definitively understood. Therefore, in the following discussion $\beta$ will simply be taken to be of order 1 within the plasma-field mixing layer.

The width of the plasma-field mixing layer relative to the size of the ion gyroradius proves to be a key parameter for driving the LHD instability in its linear phase. This ratio also characterizes the microturbulent resistivity in the nonlinearly evolved turbulent phase. For these reasons, it is important to ascertain whether there is an initial plasma-field mixing layer width that might be immediately formed during the propellant injection process, and if so, to ascertain its characteristic thickness. Moreover, this initial plasma-field mixing layer width defines the initial condition for further resistive broadening as the propellant moves downstream, no matter whether that diffusion be classical or anomalous. Thus, the first order of business is to identify the width of the plasma-field mixing layer that naturally occurs when injected plasma first impacts magnetic flux. 


\subsection{Interface Width in Breech of Nozzle}

We preview the results by emphasizing that there must be an irreducible initial plasma-field mixing layer thickness engendered at the very injection of plasma into the external magnetic field. Moreover, it cannot be understood from within the resistive MHD model. Hall effects and kinetic (individual particle) effects are responsible for its formation, which therefore would be missed in standard resistive MHD simulations such as those performed by the MACH2 code. Modeling of the initial mixing layer in the breech of the nozzle therefore comprises the topic of this section.

Older theories of the interface width separating an impacting plasma from an external magnetic field, self-consistently taking into account the magnetic shielding by the plasma particles themselves, predicted that the width would be on the order of the hybrid electron-ion gyroradius $\sqrt{a_{i} a_{e}}$. This collisionless model is known as the Ferraro-Rosenbluth sheath. (See Ref. 16 and references therein.) However, Peter, Ron, and Rostoker demonstrated in Reference 16 that the sheath would rapidly disintegrate as an entity because of flute-type instabilities with wavelengths somewhat larger than the sheath thickness. Moreover, the disintegration would occur very quickly. The growth rate can be inferred to be $\sim \sqrt{\omega_{c i} \omega_{c e}}$, the lower hybrid gyrofrequency. The action of these fast flute instabilities is believed to be the reason that the Ferraro-Rosenbluth sheath has never been observed. In this subsection, we develop a model with early turbulent collisions for the electrons and later classical or anomalous electron-ion collisions, for a much larger interface width, $\sim c / \omega_{p i}$. Such a sheath width of impacting plasma has been observed by Ripin et al. (Ref. 17).

A heuristic picture will be developed of the initial plasmafield mixing layer thickness produced by injected propellant in the breech of the nozzle. The result indicates that, on the basis of fundamental physical processes, the initial thickness is on the order of the ion inertia length $c / \omega_{p i}$. Here, $c$ is the

speed of light in vacuum and $\omega_{p i}$ is the ion plasma frequency. For conciseness, no detailed attempt will be made to derive exact numerical factors, but the length scale of the initial quasi-radial plasma-field transition will be established (where "initial" refers to conditions in the breech of the nozzle that are immediately experienced by a given element of injected plasma, but a macroscopic stationary flow is allowed).

The interaction between newly injected propellant plasma and the magnetic nozzle field may be characterized to first order as the inability of "new" electrons to freely cross the nozzle's magnetic flux surfaces because of their diminutive electron mass. That is, the electrons have not yet had time to collisionally diffuse any significant quasi-radial distance across the magnetic field in the manner discussed earlier. So in this simplified model, the electrons are, at first, treated as collisionless during the short initial ion gyrotime of interest.

The individual ions, on the other hand, make sizeable quasi-radial excursions across the flux surfaces into the region of strong magnetic field because of their much greater mass. Thus, a quasi-radial space-charge electric field $E_{x}$, which limits the ion excursions, is established in the mixing layer. It points radially inwards towards the core plasma. The nozzle longitudinal magnetic field $B_{\text {long }}$ is screened out of the core plasma according to a characteristic spatial transition of width $\mathrm{d} x$. By Ampere's law, it arises from the net azimuthal current layer $J_{y} d x$ carried by charged particle species in the interface region.

Our model focuses on a short time interval after injection of a given plasma element. This is a time interval so short that only electrons can carry the azimuthal current - for the reasons explicated below. A motional azimuthal electric field associated with the quasi-radial fluid velocity $V_{x}$ expanding across a longitudinal magnetic field $B_{\text {long (local coordinates) }}$ is neglected. This simplification proves to correspond to a highly sub-Alfvenic expansion across the magnetic field, $V_{x}$ $\ll V_{A}$. See the justifying remarks in Section 3.1, "Interface Width in Breech of Nozzle." Also, see Reference 17.

We consider a planar interface model having the nozzle magnetic field $B$ in the z-direction (into the page), the quasiradial $x$-direction pointing vertically upwards across flux surfaces towards the region of strong magnetic field, and the $y$-direction (the azimuthal direction) pointing horizontally rightwards. The nozzle magnetic field becomes negligible towards the negative $\mathrm{x}$-direction, into the core plasma. Also, the space-charge electric field $\mathbf{E}$ points in the negative $\mathrm{x}$-direction, and is perpendicular to the magnetic field (see Fig. 3).

The ions cross the magnetic field more easily than the electrons, so the outer part of the boundary layer is ion rich, whereas the inner part of the boundary layer is electron rich. These conditions set up a strong space-charge electric field, which serves to give the electrons a guiding center drift, in conjunction with the presence of the magnetic field.

During one ion gyroperiod, only the electrons experience a guiding-center drift, because of their relatively rapid gyrations. This drift is to the right, in the direction of $\mathbf{E} \times \mathbf{B}$ (clockwise in terms of the azimuthal angle). This is equivalent to a current flow to the left, which is in the direction to weaken the internal magnetic field and to strengthen the external magnetic field.

We consider only very early times while following newly injected plasma, during which "new" ions within the magnetic field interface can barely complete a significant fraction of a gyroperiod. (For hydrogen ions entering into a magnetic field of $0.5 \mathrm{~T}$, one gyroperiod is $0.1 \mu \mathrm{s}$, whereas the transit time through the meter-long nozzle is tens of microseconds for $V_{/ /} \approx 10^{5} \mathrm{~m} / \mathrm{s}$.) 
Magnetic field region (plasma free)

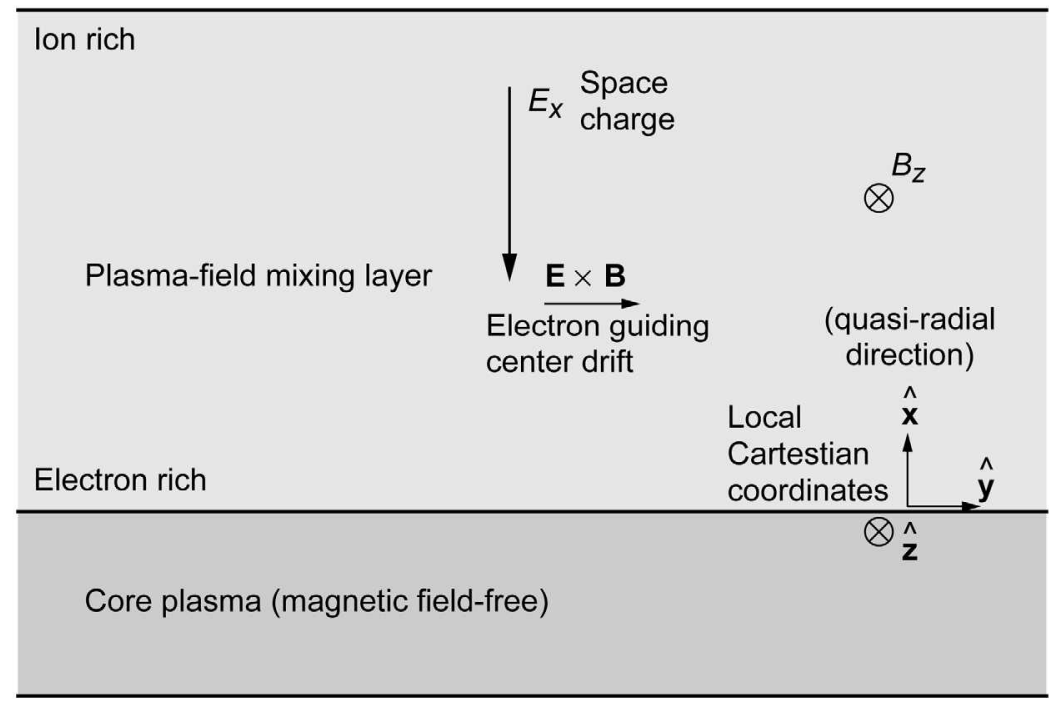

Figure 3.- Initial plasma-field mixing layer produced by propellant injection into breech (view looking downstream).

During this short time interval, the new ions cannot express a macroscopic azimuthal current - in the absence of an azimuthal electric field (again, see Sec. 3.1). The reason is that a macroscopic azimuthal ion current would be the net cooperative effect of individual ion guiding-center drifts and magnetization current loops (due to partially uncancelled gyro-orbits in a density gradient). These individual particle mechanisms, by their very nature, require many ion gyroperiods to become macroscopically evident. They are therefore not available to the ions during an early time increment lasting less than one ion gyrocycle after injection while following a given plasma element downstream. By the way, this view of the situation implicitly defines the local reference frame with respect to azimuthal velocity, in that the ions are assumed injected without any macroscopic rotational motion. In other words, at injection there is no azimuthal beam of ions in the lab frame of reference. The electrons, on the other hand, do exhibit very many gyrocycles during this first ion gyration and so can indeed manifest macroscopic azimuthal currents early on.

To simplify the model, we suppose that the electrons are collisionless (a restriction removed later) and have negligible pressure (also removed later). Then, the electrons carry an azimuthal macroscopic current only as the result of an $E / B$ azimuthal guiding-center drift velocity (in mks units). This drift, unlike the other current generating processes, does not need significant electron pressure. $E / B$ drift results from nonuniform curvature of a gyro-orbit. During energy gain from $-E$, the electron's orbit acquires a greater radius of curvature.
Over the part of the orbit against $-E$, energy is lost producing a smaller radius. The trajectory is a cycloid, a gyrocircle with a moving center having velocity $E / B$. If the temperature $T_{e}$ were very small then the gyrocircle would be very small, but the description still applies.

This temporary exclusion of electron pressure precludes other current-generating individual particle effects, namely magnetization currents and grad- $B$ guiding-center drifts. The simplifying assumption of no field-line curvature in the breech also precludes "curvature drifts" of the electron's guiding center. At any rate, the latter drift also vanishes in the zero-electron-pressure approximation. (From the way the fluid equations are derived from more detailed models, we know that the electron fluid equations - with electron temperature included - automatically incorporate the various individual-particle effects, as regards production of macroscopic currents.)

In view of the zero-electron-pressure assumption, the electron macroscopic momentum equation in mks units is simply

$$
0=-n_{e} q\left(\mathbf{E}+\mathbf{V}_{e} \times \mathbf{B}\right)
$$

where $\mathbf{V}_{e}=\mathbf{E} \times \mathbf{B} / B^{2}$ is the guiding-center (macroscopic fluid) drift velocity vector of the electrons, of number density $n_{e}$. The above equation, in component form, where $B_{z}=B_{\text {long }}$, is equivalent to 


$$
E_{x}=\frac{J_{y} B_{z}}{n_{e} q}
$$

where $J_{y}=-n_{e} q V_{y e}$ is the azimuthal current density carried by the electrons in the plasma-field mixing layer, and $V_{y} e$ is positive to the right. This current is driven such as to cancel the vacuum magnetic flux within the plasma and to increase it outside of the plasma. Since the longitudinal flux cannot be created or destroyed over a short time interval within the volume enclosed by the conducting wall (the coil), the magnetic flux is pushed aside or displaced by the injected plasma.

By means of Ampere's law, which reads $\mu_{0} \mathbf{J}=\nabla \times \mathbf{B}$ (or also $\mu_{0} J_{y}=-\partial_{x} B_{z}$ ), Equation (101) for the space-charge field can be written as

$$
E_{x}=-\left(\frac{1}{n_{e} q}\right) \partial_{x} \frac{B_{z}^{2}}{2 \mu_{0}}
$$

This result proves to be key to estimating the thickness of the plasma-field mixing layer in the nozzle magnetic field. It is based on the azimuthal current being carried only by the electrons.

We now have to consider the time-dependent position, that is, the trajectory $\mathbf{r}(t)$, of an individual ion that is incident on the plasma-field mixing layer. It can be shown that the inertial force on the individual ion from radial deceleration of the plasma fluid is small compared to the force on the individual ion from the space-charge electric field, provided that the radial fluid expansion velocity is highly subAlfvenic. See Appendix $F$ for the derivation. The single particle's velocity vector is $\mathrm{v}_{i}=\mathrm{dr} i \mathrm{~d} t$. The momentum balance equation of an ion, in the plane perpendicular to $\mathbf{B}$, reads

$$
m_{i} \frac{\mathrm{dv}_{\perp i}}{\mathrm{~d} t}=q\left(\mathbf{E}+\mathbf{v}_{\perp i} \times \mathbf{B}\right)
$$

Here, the velocity vector of the ion is partitioned as $\mathrm{v}_{i}=\mathrm{v}_{\perp i}+v_{z i} \mathbf{B} / B$. The space-charge electric field vector $\mathbf{E}$ is in the local $\mathbf{x}$-direction, and the magnetic-field vector $\mathbf{B}$ is in the local z-direction. The scalar product ("dot product") of Equation (103) with $\mathbf{v}_{\perp i}$ then leads to a statement of energy balance in the form

$$
\frac{\mathrm{d}\left(1 / 2 m_{i} v_{\perp i}^{2}\right)}{\mathrm{d} t}=q E_{x} v_{x i}
$$

Equation (104) shows that the magnetic field does no work on the ion, and that the work done on the particle by the $\mathrm{x}$-directed electric field affects the total perpendicular energy of the particle, not merely the energy associated with the quasi-radial velocity $v_{x} i$. That is, in Equation (104) $v_{\perp i}^{2}=v_{x i}^{2}+v_{y i}^{2}$. Therefore, to make use of this energy balance equation we also need an independent equation for the azimuthal velocity component $v_{y}$, furnished by the azimuthal component of the momentum balance Equation (103):

$$
m_{i} \frac{\mathrm{d} v_{y i}}{\mathrm{~d} t}=-q v_{x i} B_{z}
$$

It now proves useful to relinquish the time variable, utilizing instead the $x$-coordinate of the particle's quasi-radial position as the independent variable. Noting that $v_{x i}=\mathrm{d} x / \mathrm{d} t$, the energy balance Equation (104) becomes

$$
\frac{\mathrm{d}}{\mathrm{d} x}\left(\frac{m_{i} v_{x i}^{2}}{2}+\frac{m_{i} v_{y i}^{2}}{2}\right)=q E_{x}
$$

Likewise, the azimuthal momentum Equation (105) becomes

$$
m_{i} \frac{\mathrm{d} v_{y i}}{\mathrm{~d} x}=-q B_{z}
$$

Multiplying through by $v_{y} i$ in Equation (107) shows that Equation (106) can also be written as

$$
\frac{\mathrm{d}}{\mathrm{d} x}\left(\frac{m_{i} v_{x i}^{2}}{2}\right)=q\left(E_{x}+v_{y i} B_{z}\right)
$$

Hence, energy balance does indeed apply to just the quasiradial x-component of ion motion, but only from within the instantaneous azimuthal-moving frame of the ion. Note the appearance of the radial electric field in the moving frame.

The best way to convey our approach to the problem of the initial thickness of the edge-plasma boundary layer is to begin by integrating the azimuthal momentum equation, Equation (107). The injected plasma has no directed azimuthal velocity. Thermal ions incident on the plasma-field mixing layer then will be equally likely to have positive and negative values of $v_{y}$. As a representative ion, therefore, we choose an intermediate example for which the azimuthal velocity at the core region is $v_{y} i$ core $=0$. The maximum radial extent of this particular trajectory is called $\Delta$, which is the quantity to be determined. Presumably, this particular $\Delta$ lies in the midst of the distribution of $\Delta$-values generated by internal ions incident on the plasma-field mixing layer at all possible angles. 
Equation (107) is integrated from $x=0$ to $x=\Delta$. The final azimuthal velocity is written as $v_{y} i \Delta$. The result of this integration then reads

$$
v_{y i \Delta}=-\left\langle\omega_{c i}\right\rangle \Delta
$$

wherein $\left\langle\omega_{c i}\right\rangle=q\left\langle B_{z}\right\rangle / m_{i}$ is the radial average of the ion gyrofrequency over the considered trajectory segment. Note that the appearance of the radial average magnetic field is mathematically correct and required. The average magnetic field within the plasma-field mixing layer is identified as roughly half of the outer magnetic field: $\left\langle\omega_{c i}\right\rangle=\omega_{c i} / 2$. Thus $B_{z}$ and $\omega_{c i}$ without brackets now indicate the stronger magnetic field out beyond the plasma-field mixing layer. Then (109) becomes

$$
v_{y \Delta i}=-\frac{\omega_{c i} \Delta}{2}
$$

Now the energy balance Equation (106) can be integrated from $x=0$ to $x=\Delta$. The impacting radial velocity is denoted by $v_{x} i$ core, and the final radial velocity at the outermost extent of the trajectory segment is clearly $v_{x i \Delta}=0$. Integrated energy balance for an individual ion over the entire specified trajectory segment then can be expressed as

$$
-\frac{m_{i} v_{x i \text { core }}^{2}}{2}+\frac{m_{i} v_{y i \Delta}^{2}}{2}=q\left\langle E_{x}\right\rangle \Delta
$$

Equation (111a) slightly rearranged,

$$
\frac{m_{i} v_{x i \text { core }}^{2}}{2}=-q\left\langle E_{x}\right\rangle \Delta+\frac{m_{i} v_{y i \Delta}^{2}}{2}
$$

states that the incident kinetic energy of the outward moving ion, $1 / 2 m_{i} v_{x i \text { core }}^{2}$, is partly depleted by doing work $-q\left\langle E_{x}\right\rangle \Delta$ against the inward-directed space-charge electric field (recall that $E_{x}$ is negative). The leftover kinetic energy at the end of the considered trajectory segment is just $1 / 2 m_{i} v_{y i \Delta}^{2}$. Again, the appearance of the radial average of the electric field $\left\langle E_{x}\right\rangle$ is indeed mathematically correct and required.

The expression for the electric field in Equation (102) is used in Equation (111b), which then becomes

$$
\frac{m_{i} v_{x i \text { core }}^{2}}{2}=q \Delta\left\langle\left(\frac{1}{n_{e} q}\right) \partial_{x}\left(\frac{B^{2}}{2 \mu_{0}}\right)\right\rangle+\frac{m_{i} v_{y i \Delta}^{2}}{2}
$$

In the further interpretation of Equation (112), the extremely powerful drive to quasi-neutrality is recognized at the envisioned plasma density $10^{15} \mathrm{~cm}^{-3}$ (provided that the space-charge field does not get shorted out).

Accordingly, we assume quasi-neutrality in the transition layer, namely $n_{e} \approx n_{i}=n$ (for hydrogen, with $\Xi=1$ ). Thus, $n_{i}-n_{e} \mid \ll n_{i}$. This assumption does not preclude a significant (but not unrealistically huge) space-charge electric field that maintains quasi-neutrality. A specific knowledge of the anomalous microturbulence that instigates the early transition to quasi-neutrality in the interface region is not needed for the estimate of the early width of the interface.

Note: A preliminary transient phase of virulent microturbulence must exist, driven by a huge space-charge electric field, which facilitates rapid transport of electrons across flux surfaces as they follow ions in an attempt to preserve neutrality. The main text assumes that this preliminary phase is already completed so that quasi-neutrality is preserved. Without this preliminary adjustment, the space-charge field would remain huge. For example, if an ion density of $10^{15} \mathrm{~cm}^{-3}$ were displaced a distance of only $0.001 \mathrm{~cm}$ beyond the electron boundary, it would create an electric field of $2 \mathrm{MV} / \mathrm{cm}$, with severe consequences. An alternative possibility is that the electron boundary remains absolutely fixed and that the ions are allowed to extend only a thermal Debye length $(0.00016 \mathrm{~cm}$ at $50 \mathrm{eV})$ beyond the electron boundary into the magnetic field. This also seems unrealistic and contradicts experimental observations. It seems to be necessary that there is a preliminary draconian adjustment of the electrons' distribution upon plasma injection. This anomalous electron turbulence is shown to be necessary in Appendix F, where classical mobility and collisional drift cannot cause electrons to traverse the required ion penetration depth of an order of a fraction of an ion gyroperiod.

An approximate estimate of the average term $\left\langle\left(1 / n_{e} q\right) \partial_{x}\left(B^{2} / 2 \mu_{0}\right)\right\rangle$ in Equation (112) can be made by simply using intermediate values of the quantities therein, which does not call upon any detailed profiles within the plasma-field mixing layer. The value of $n_{e} \approx n$ in the denominator of Equation (112) ranges from its core value to a much smaller value over the plasma-field mixing layer width $\Delta$. Its average, therefore, is simply interpreted as half of the number density $n_{\text {core }}$ in the core plasma. Also, we interpret the average spatial derivative of magnetic pressure in Equation (112) as the outer value of magnetic pressure divided by $\Delta$. (The neglected inner value of magnetic pressure is significantly smaller.) 
The characteristic radial spatial decrease of $B^{2}$ into the plasma should be governed approximately by the same layer width $\Delta$ as governs the characteristic radial outward decrease of plasma density (associated with the radial protrusion of the ion trajectories). This behavior of density and magnetic field profiles is consonant with the constancy of total (plasma plus magnetic) pressure across the plasma-field mixing layer (see Appendixes $\mathrm{G}$ and $\mathrm{H}$ ).

Such rudimentary partitioning of the whole quantity $\left\langle\left(1 / n_{e} q\right) \partial_{x}\left(B^{2} / 2 \mu_{0}\right)\right\rangle$ in Equation (112) proves to be not far numerically (within a factor of 2) from the exact answer obtained with specific sample profiles. The detailed profiles within the plasma-field mixing layer are, of course, unknown. However, for example, with exponentially decreasing $n(x)$ and exponentially increasing $[B(x)]^{2}$ over width $\Delta$ (qualitatively consistent with pressure balance across the layer), one obtains a numerical factor 1.17 instead of 2 in Equation (113) below. Moreover, a square root is eventually extracted to find the width $\Delta$, which finally yields a comparative error of only about 30 percent.

Using just the above rudimentary procedure then, after multiplying by $2 / m_{i}$ Equation (112) can be reduced to

$$
v_{x i \text { core }}^{2}=2 V_{A}^{2}+v_{y i \Delta}^{2}
$$

Here, the square of the Alfven speed $V_{A}^{2}=B^{2} / \mu_{0} n m_{i}$ contains the outer magnetic field beyond the plasma-field mixing layer, and the inner number density of core plasma. We suppress the subscripts that would have supplied those meanings.

Equation (110) for $v_{y \Delta i}$ is now used in Equation (113), together with the key identity

$$
\frac{V_{A}}{\omega_{c i}}=\frac{c}{\omega_{p i}}
$$

The result reads

$$
v_{x \text { core } i}^{2}=\left(2 V_{A}^{2}\right)\left(1+\frac{\omega_{p i}^{2}}{8 c^{2}} \Delta^{2}\right)
$$

This result is based upon energy and momentum balance for an individual ion's trajectory, together with the relation of the radial space-charge electric field to the azimuthal electron current, obtained from the fluid electron's radial momentum equation. No overt connection is invoked here or below with the actual temperature of the ions or with the thermal gyroradius of an ion. This salient point will be emphasized again below.
Note that the $\Delta$ estimated here depends, in principle, on the particular slice of the ion's incident velocity distribution used to make the calculation. Although this particular $\Delta$ is assumed to be representative of the plasma-field mixing layer width, that assumption is not rigorously tested in this report.

We now heuristically consider the trajectory of an individual ion as the ion enters at perpendicular incidence and proceeds into the plasma-field mixing layer. Incident azimuthal velocity $v_{y \text { core } i}=0$, but incident parallel (to B) velocity $v_{z \text { core } i}$ is arbitrary. If it undergoes roughly one-fourth of a gyrocircle in the average magnetic field in the plasma-field mixing layer, such a trajectory segment is expected to be approximately commensurate with the time needed for that ion to reach its maximum radial protrusion beyond the core plasma, denoted by $\Delta$. Since the final radial velocity vanishes, the average radial velocity over the quarter circle is taken to be half of the incident radial velocity. Then one obviously has

$$
\Delta \approx \frac{1}{2} v_{x i \text { core }} \tau_{i 1 / 4} \approx \frac{1}{2} v_{x i \text { core }} \frac{\pi / 2}{\left\langle\omega_{c i}\right\rangle}=v_{x i \text { core }} \frac{\pi / 2}{\omega_{c i}}
$$

In Equation (116), time $\tau_{i 1} 1 / 4$ is taken as one-quarter of the ion gyroperiod in the average magnetic field within the plasma-field mixing layer. Again, the average magnetic field is set to half the outer magnetic field, so the ion gyrofrequency $\omega_{c i}$ without brackets in Equation (116) is understood to contain the outer magnetic field.

Next, we calculate $\Delta^{2}$ from Equation (116) and use Equation (115) for $v_{x i \text { core }}^{2}$ and the identity in Equation (114). In that manner we easily obtain the following equation for $\Delta_{\text {norm }}^{2}=\left(\Delta \omega_{p i} / c\right)^{2}$ where $\Delta_{\text {norm }}$ denotes the nondimensional version of $\Delta$ measured in units of the ion inertia length $c / \omega_{p i}$ (and $\omega_{p i}$ refers to $\left.n_{\text {core }}\right)$ :

$$
\Delta_{\text {norm }}^{2}=\frac{\pi^{2}}{2}\left(1+\frac{\Delta_{\text {norm }}^{2}}{8}\right)
$$

The term $\Delta_{\text {norm }}^{2} / 8$ represents the final azimuthal kinetic energy that ultimately remains of the ion's incident radial kinetic energy, after subtracting the work done by the ion against the space-charge field. The term $\Delta_{\text {norm }}^{2} / 8$ in Equation (117) proves to be comparable to the first term (i.e., $\approx 1$ ). The solution of Equation $(117)$ is $\Delta_{\text {norm }}^{2} \approx 13$; then, $\Delta_{\text {norm }} \approx 3.6=\Delta \omega_{p i} / c$. 
Use of the specific sample profiles mentioned above in evaluating the average space-charge electric field suggest the possibility that the coefficient of the first term in Equation (113) (i.e., 2) should perhaps have been "1." Then, instead of Equation (117),

$$
\Delta_{\text {norm }}^{2}=\frac{\pi^{2}}{4}\left(1+\frac{\Delta_{\text {norm }}^{2}}{4}\right)
$$

with the solution $\Delta_{\text {norm }} \approx 2.6$.

These approximate model results paint a definitive picture. Consider a newly injected plasma element near the interface between the plasma and the magnetic nozzle flux, which is followed downstream at early times not exceeding about one-fourth of an ion gyroperiod. The extent of a representative ion protrusion out of the plasma element into the (displaced) magnetic flux is a few ion inertia lengths as characterized by the core-plasma number density; that is,

$$
2.6\left(\frac{c}{\omega_{p i}}\right) \approx \Delta \approx 3.6\left(\frac{c}{\omega_{p i}}\right)
$$

Our heuristic model cannot guarantee the exact numerical factor. Except possibly for the numerical factor, this result for the plasma's edge-layer thickness is dependent neither on the incident radial velocity of the ion nor on the strength of the external confining magnetic field. It depends solely on the ion number density in the bulk plasma.

The constraints built into the model that yields this result are as follows: First, quasi-neutrality within the plasma-field mixing layer is achieved immediately in a preliminary transient adjustment involving microturbulence and is maintained thereafter. The almost-massless electrons pulled into the magnetic flux by quasi-neutrality have become attached to the magnetic flux. Second, the electron current layer shields the interior plasma from the magnetic field practically from the outset of injection of the considered plasma element (after several electron gyroperiods). Third, the ion component makes practically no contribution to the azimuthal current layer. Finally, a cautionary note is that the calculated $\Delta$ was obtained here by considering a special restricted class of individual ion trajectories selected out of the thermal-ion distribution, namely those that enter the plasmafield mixing layer without azimuthal velocity. A rigorous calculation of $\Delta$ requires an average over the entire incidention velocity distribution.

Note that the edge-plasma boundary layer thickness $\Delta$ as calculated here also characterizes the inward-radial decay of $B^{2}$, according to Equation (112) and the associated discussion. Hence, the characteristic decay distance of $B$ itself into the plasma must be $2 \Delta$, and $\Delta \approx 2 c / \omega_{p i}$ to $3 c / \omega_{p i}$ (comparable to Eq. (119)). The ion inertia length is about $1 \mathrm{~cm}$ for a hydrogen-plasma having number density $10^{15} \mathrm{~cm}^{-3}$.

There is indeed experimental evidence (Ref. 17) for $c / \omega_{p i}$ to be the length scale for the plasma-field transition layer produced when injected plasma first impacts upon and displaces the ambient magnetic field. The account in Reference 17, however, does not provide a clear and definitive understanding of this feature. Although Reference 17 addresses laser-produced plasma expansion into an existing magnetic field, the physics issues therein are generic and relevant to injection of plasma into a magnetic nozzle.

The above results for the width of the plasma-field interface of newly injected plasma can be very simply obtained from a reduced model, within about a factor of 2 . The reduced model begins by assuming that ions of only a single outward quasi-radial velocity are initially incident on the interface, $v_{x \text { core } i} \neq 0$ and $v_{y}$ core $i=0$, with $v_{z \text { core } i}$ being arbitrary but following the plasma in the reference frame of $v_{z}$ core $i$. It also assumes, as before, that the electron current layer is responsible for magnetic shielding of the interior plasma. A key assumption is that there is pressure balance across the interface. The electron pressure is still neglected in this reduced model.

Consider the rate at which outward ion momentum flows into the interface, per unit area, during the first quarter to half gyroperiod, before the ions have been fully reflected by the outer magnetic field: It is the particle flux multiplied by the momentum of a particle; namely, $\left(n_{\text {core }} v_{x \text { core } i}\right)\left(m_{i} v_{x \text { core } i}\right)$ $=\rho_{\text {core }} v_{x \text { core } i}^{2}$. Balancing this outflux of initial radial ion momentum against the inward magnetic pressure immediately yields $v_{x \text { core } i}=V_{A} / \sqrt{2}$, in which the Alfven velocity is evaluated with the outer magnetic field and the inner mass density. The outward fluid velocity would be smaller with a full angular velocity distribution.

The distance beyond the core where an ion starts to turn around (radial velocity $\rightarrow 0$ ) during one-quarter of a gyroperiod in the average magnetic field in the plasma-field mixing layer is

$$
\begin{aligned}
\Delta=\left\langle v_{x i}\right\rangle \tau_{i 1 / 4} & \approx \frac{1}{2} v_{x \text { core } i} \frac{\pi / 2}{\left\langle\omega_{c i}\right\rangle} \\
& \approx \frac{\pi}{2} \frac{v_{x \text { core } i}}{\omega_{c i}}=\frac{\pi}{\sqrt{8}} \frac{V_{A}}{\omega_{c i}}=\frac{\pi}{\sqrt{8}} \frac{c}{\omega_{p i}}
\end{aligned}
$$

Here as before, the average radial velocity in the layer is taken as half the incident velocity, and the average magnetic field in the layer has been taken as half the outer magnetic field. Because electrons have been pulled out into this layer by the forces that maintain quasi-neutrality, they will have become attached to magnetic flux in the layer. Thus, the 
evolution of layer width resembles a ratchet process that cannot be reversed.

Recall the more detailed model that dealt with the energy and momentum balance of an ion's trajectory in the spacecharge electric field and the shielded magnetic field. It apparently gives a somewhat larger value for the width of the interface: namely $2.6 c / \omega_{p i}$ to $3.6 c / \omega_{p i}$ (comparable to Eq. (119)). Also recall that the corresponding decay length of the magnetic field into the core plasma is predicted to be twice this value.

It is important to make a clarification regarding the above model. A motional azimuthal electric field has been neglected at the outset that otherwise would have been produced by a radial fluid velocity of injected plasma expanding across the longitudinal magnetic flux. In principle, the ions in the interface region can respond to this azimuthal electric field during their initial quarter gyroperiod. Thereby they can contribute to the azimuthal macroscopic current provisionally assumed carried only by the electrons. It can be shown, however, that the ion contribution would be minor, provided that the plasma-fluid's initial quasi-radial expansion velocity across B is highly sub-Alfvenic.

Specifically, it can be demonstrated that the azimuthal fluid velocity component $V_{y} e$ of the current-carrying electrons is of order $V_{A}$. In contrast, the azimuthal fluid velocity $V_{y i}$ of the ions in response to the motional electric field $E_{y}=-V_{x i} B_{z}$ during the first quarter gyroperiod is much smaller. In particular, $V_{y} i$ proves to be merely on the order of the radial ion-fluid velocity $V_{x i}$. See Appendix $\mathrm{F}$ for the derivation of these results. The massless electron response to $E_{y}$ merely would be a radial $\mathbf{E} \times \mathbf{B}$ guiding center drift. The electron contribution to the azimuthal current would not be directly affected by the azimuthal electric field. Therefore, if the plasma's radial expansion velocity $V_{x i}$ is highly subAlfvenic, that is, if $V_{x i} \ll V_{A}$, then $V_{y i} \ll<V_{y e}$ and the ions cannot make a significant contribution to the macroscopic azimuthal current. The electrons carry practically all the azimuthal current.

The reason that it is possible in principle for the fluid plasma's radial expansion velocity to be sub-Alfvenic at the plasma interface with the magnetic field is as follows. After a brief sub-Alfvenic dynamical adjustment in the strong magnetic field limit, described in Appendix $\mathrm{H}$, further radial expansion of the ion fluid involves the ions having to drag reluctant electrons across longitudinal magnetic flux. The resulting expansion velocity principally depends on the nature of the resistivity or the microturbulence that facilitates crossfield electron transport, even right after injection. If, on the other hand, the plasma's initial quasi-radial-expansion fluid velocity (macroscopic velocity) were close to the Alfven speed in the plasma-field interface region, then it would be important to incorporate into the model the corresponding motional azimuthal electric field and the ion response to it.
That has not been done in the present report, which definitely assumes a sub-Alfvenic expansion across the magnetic flux.

We now remove the two restrictive assumptions that were made on the electrons. These were the neglect of electronion collisions and the neglect of electron pressure.

If electron-ion collisions are included (but still with neglect of electron pressure), then the electron-fluid momentum equation can easily be solved for the electron-fluid velocity $\mathbf{V}_{e}$, which then reads

$$
\mathbf{V}_{e}=-\Omega\left(\frac{1}{1+\Omega^{2}}\right) \frac{\mathbf{E}}{B}+\Omega^{2}\left(\frac{1}{1+\Omega^{2}}\right) \frac{\mathbf{E} \times \mathbf{B}}{B^{2}}
$$

Here, $\Omega=\omega_{c} e^{/ v_{e}}$ is the ratio of the electron gyrofrequency $q B / m_{e}$ to the collision frequency (possibly anomalous) of a representative electron with the ions. In the limit $\Omega \rightarrow 0$, the second term, the azimuthal $E / B$ drift velocity term, disappears. The remaining first term reduces to the usual electron drift mobility term along the electric field, without a magnetic field; namely, $-\left(q / m_{e} v_{e}\right) \mathbf{E}$.

For parameters of interest to us, however, $\Omega^{2} \gg 1$. For example, with the hydrogen plasma particle number density $10^{15} \mathrm{~cm}^{-3}$, a plasma temperature of $50 \mathrm{eV}$, and a magnetic field strength of order $0.5 \mathrm{~T}(5000 \mathrm{G})$, one has $\omega_{c e} \approx$ $10^{11} \mathrm{rad} / \mathrm{s}$. In contrast, the classical Spitzer collision frequency is $v_{e} \approx 10^{8} \mathrm{~s}^{-1}$. Then $\Omega=1000$ and $\Omega^{2}=10^{6}$. It is immaterial to the argument whether 0.2 or $0.5 \mathrm{~T}$ is used here. Even if the electron collision frequency were anomalously large by a factor of 10 or 100 , it would still be the case that $\Omega^{2} \gg 1$. For large $\Omega^{2}$, Equation (121) reduces to

$$
\mathbf{V}_{e}=-\left(\frac{1}{\Omega}\right) \frac{\mathbf{E}}{B}+\frac{\mathbf{E} \times \mathbf{B}}{B^{2}}
$$

With large $\Omega$, the drift velocity of the electrons along the quasi-radial electric field (first term) becomes very small compared with the azimuthal $E / B$ drift velocity (second term). This result is just what is necessary to carry out the above simplified derivation and estimate of the thickness $\Delta$ of the edge-plasma transition layer.

The radial diffusion distance of electrons due to their coulomb collisions with ions during one-fourth of an ion gyroperiod can easily be calculated, as can the radial mobilitydrift-distance during that same time interval from the first term of Equation (122). The former distance is approximately $\Omega^{-1 / 2} c / \omega_{p} ;$ the latter is $\Omega^{-1} c / \omega_{p i}$ (see Appendix F for the derivation). It is still assumed that the transient subphase that preserves quasi-neutrality has already occurred. The quarter ion-gyroperiod diffusion result $\Omega^{-1 / 2}\left(c / \omega_{p i}\right)$ compares with the resistive-MHD boundary-layer diffusion 
as evolved along flow distance $L_{b t}$ (Eq. (77)), which is approximately $\delta \approx \Omega^{-1 / 2}\left(L_{b i} c / \omega_{p i}\right)^{1 / 2}$.

Both of the above distances are small in comparison to the layer width $c / \omega_{p i}$ when $\Omega \gg 1$. Thus, the effects of collisional diffusion and mobility-drift of electrons in the radial direction are not important, for the electrons then would travel radially only a small fraction of the layer thickness during one-fourth of an ion gyroperiod. This is the case although there are many collisions of an average electron during one ion gyroperiod. Therefore, the dominant motion of electrons still is governed by the azimuthal $E / B$ drift velocity, which is what enabled the estimate of $c / \omega_{p i}$ for the transition layer thickness.

Moreover, the same qualitative picture would remain marginally valid even if the electron collision frequency $v_{e}$ were several hundred times larger and on the order of the electron gyrofrequency $\omega_{c e}$, thus making $\Omega \approx 1$. Hence we reach a different conclusion with respect to the effect of electron collisions than did Reference 17, which failed to emphasize the importance of the electron's $E / B$ drift in determining the thickness of the plasma-field transition layer.

The above remarks addressing the effects of electron collisions can easily be extended to include electron pressure. Instead of Equation (122) one finds

$$
\mathbf{V}_{e}=-\left(\frac{1}{\Omega}\right) \frac{\mathbf{E}_{e f f}}{B}+\frac{\mathbf{E}_{e f f} \times \mathbf{B}}{B^{2}}
$$

where $\mathbf{E}_{\text {eff }}=\mathbf{E}+\left(n q_{e}\right)^{-1} \nabla P_{e}$. Again, large $\Omega$ ensures the dominance of the azimuthal electron-fluid velocity, the second term in Equation (123).

However, the second term in (123) now contains $\mathbf{E}_{\text {eff }}$ instead of just $\mathbf{E}$, so there are some differences in detail as regards both the derivation and the result for the thickness of the plasma-field transition layer. For example, the negative electron pressure gradient adds its effect to the negative space-charge electric field so as to enhance the azimuthal electron-fluid velocity, augmenting magnetic shielding by the electron current layer. One expects that this current enhancement will act to decrease the thickness of the plasma-field mixing layer. Such calculations are performed in Appendixes $\mathrm{G}$ and $\mathrm{H}$.

The derivations in Appendixes $\mathrm{G}$ and $\mathrm{H}$ include the fluidelectron pressure gradient and for simplicity allow the electrons to have the same temperature as the ions. Also, these temperatures are assumed to be uniform within the plasmafield mixing layer. In addition, Appendix $\mathrm{H}$ explicitly includes the fluid-ion pressure gradient, and moreover, the assumption of a static plasma-field mixing layer is relaxed. (Assuming that the expanding plasma element has made the initial adjustment to quasi-neutrality when it first encounters the external magnetic field region, then there is no inconsistency of quasi-neutrality with the small mobility and drift displacements of the electrons.)

A more rigorous treatment of radial averaging of the electric field is carried out in Appendix G, a somewhat different averaging treatment independent from those described above. Again, specific profile shapes within the plasma-field mixing layer are not essential restrictions to make the calculations. Then, instead of Equation (117) or (118),

$$
\Delta_{\text {norm }}^{2}=\frac{\pi^{2}}{8}\left[\left(e-\beta_{G}\right)+\frac{1}{2} \Delta_{\text {norm }}^{2}\right]
$$

Here $e$ is the base of natural logarithms $(\approx 2.7183)$ and $\beta_{G}$ is the global $\beta$ across the plasma-field mixing layer, namely, the inner plasma pressure divided by the outer magnetic pressure. The new effect of electron pressure is now manifested by the square bracket containing the effect of the global $\beta$. As $\beta_{G}$ increases, the solution $\Delta_{\text {norm }}$ decreases, and so then does the thickness of the plasma-field mixing layer.

However, there must be overall pressure balance across a static plasma-field mixing layer, so that $\beta_{G}=1$ (see Appen$\operatorname{dix} H$ ). The solution of Equation (124) then is $\Delta_{\text {norm }} \approx 2.35$, which compares well with the earlier estimates of 3.6 and 2.6. Thus our earlier conclusion retains validity; namely, that the initial thickness of the plasma-field boundary layer (i.e., in the injection region) is a few ion inertia lengths $c / \omega_{p}$.

Early quasi-radial deceleration of injected expanding plasma is included in the calculations in Appendix H. Quasiradial deceleration is found to have no major effect on the width of the plasma-field mixing layer, provided that the quasiradial macroscopic expansion velocity of the fluid plasma is highly sub-Alfvenic (Ref. 17). This limit corresponds to a strong magnetic nozzle field.

Several important concluding remarks can be made regarding the initial width of the plasma-field mixing layer.

The plasma-field mixing layer width at the interface between plasma and magnetic field is formed in the injection region by processes not accessible to the standard resistive MHD model. It serves as the initial condition for further broadening of the layer downstream. The radially protruding collisionless ion trajectories do not at first become trapped on magnetic nozzle flux; rather, those ion trajectories return to the field-free core plasma. However, their positive spacecharge halo beyond the core pulls some edge electrons radially out of the core so as to preserve quasi-neutrality. This space-charge effect constitutes a one-way irreversible process facilitated by microturbulence driven by the initially large space-charge electric field. A detailed knowledge of that initial microturbulence is unnecessary. Since electrons are practically massless, some form of electron collisionality, be it coulomb collisions with ions or with microturbulent fluctuations, is necessary to scatter the edge electrons onto the external magnetic flux. 
There is no physical mechanism of collisions, or effective collisions, available for pulling those practically massless electrons radially back off of the magnetic flux so as to return them, macroscopically, into the core. (Such a mechanism would have to work "uphill" against the electron density gradient.) They then effectively become trapped on an external annular increment of previously expelled magnetic flux. The overall process is like a ratchet, in which a continuous supply of radially protruding ion trajectories furnishes space charge to keep the ratchet expansion process going, during the early times following injection of any given plasma element within the interface region. Note, however, that this heuristic microphysical description of interface broadening has general validity not limited to the injection region.

If the quasi-radial space-charge field were shorted out at early times after injection, by the unimpeded flow of Hall current, the edge-plasma situation would be fundamentally altered. Then, some of the edge electrons would preserve charge balance by flowing longitudinally to the backplate (as the ions partially vacate the plasma's edge layer due to the radial extent of their trajectories). The ratchet effect thereby would be circumvented. Even if the associated Hall-current circuit were not completely closed, such a global redistribution of edge-plasma electrons would drastically reduce the otherwise huge local space-charge electric field in the edge region and would at least partially mitigate the ratchet effect at the plasma-field interface.

The plasma-field mixing layer in the breech of the nozzle (sometimes called the initial boundary-layer width in this report) is characterized by the ion inertia length $c / \omega_{p}$, according to the models employed here. This initial broadening of the interface is facilitated by the above-mentioned ratchet effect. The results of these several models contain numerical multipliers suggesting that the mixing layer in the breech of the nozzle probably is a few times thicker than $c / \omega_{p i}$. This result has important consequences for the attachment of propellant to magnetic flux. For example, if the initial interface were $2 \mathrm{~cm}$ thick and the nozzle breech of injected plasma were $30 \mathrm{~cm}$ in radius, then 15 percent of the injected plasma propellant would be immediately affected. This would occur before the onset of additional adverse effects along the flow.

The width $\delta$ of the plasma-field interface-just after injection - is not only on the order of a few $c / \omega_{p i}$, but is also on the order of the ion gyroradius $a_{i}$. This follows from the relation $a_{i} /\left(c / \omega_{p i}\right)=\sqrt{\beta_{i}}$, with $\sqrt{\beta_{i}} \sim 1$ in the interface. For example, $a_{i} \delta \approx 0.5 \sqrt{\beta_{i}} \approx 1 / \sqrt{8}=0.35$, when $\delta \approx 2 c / \omega_{p i}$ and $\beta_{i} \approx 0.5$. (For a more rigorous derivation of this numerical result, see Appendix I.)

The interface itself is expected to broaden during downstream flow. An important question then arises as to whether the width of the interface exceeds $a_{i}$ downstream, as the flow approaches the nozzle's throat. This question is important because both the linear and nonlinear behavior of the LHD instability are affected by the plasma-field mixinglayer width relative to the ion gyroradius in the layer.

We will now estimate the ratio $a_{i} / \delta$ along the downstream flow. In so doing, we now formally assume the initial conditions

$$
\frac{a_{i}}{\delta} \approx 0.5 \sqrt{\beta_{i}} \text { where } \delta \approx 2 c / \omega_{p i}
$$

to hold in the breech (beginning) of the nozzle, where variables such as $n, \delta, t$, and $D$ are labeled with subscript $b$.

First, to illustrate the method, suppose that the interface is broadened just due to the action of classical resistivity, $\eta_{\mathrm{cl}} \sim 1 / T^{3 / 2}$. (Here, $T=T_{e}=T_{i}$. ) Using the broadening algorithm in simplified form (avoiding the time integral), we take $\delta^{2} \sim D_{\mathrm{cl}} t$, where $\delta$ is the width of the interface and $t$ is the longitudinal transit time for the propellant flow. Also, $D_{\mathrm{cl}}$ is the classical resistive diffusivity arising from coulomb collisions of electrons on ions, $D_{\mathrm{cl}}=\eta_{\mathrm{cl}} / \mu_{0}$ in mks units and $D_{\mathrm{cl}}=\left(c^{2} / 4 \pi\right) \eta_{\mathrm{cl}}$ in cgs units. Then $\delta^{2} \sim t / T^{3 / 2}$. The variation of the squared ratio of ion gyroradius $a_{i}$ to interface width $\delta$ can be written as

$$
\frac{a_{i}^{2}}{\delta^{2}} \sim\left(\frac{T}{B^{2}}\right) \frac{T^{3 / 2}}{t} \sim \frac{T^{5 / 2}}{n T t} \sim \frac{T^{3 / 2}}{n t} \sim \frac{1}{t}
$$

Here, we utililized radial magnetic pressure balance of plasma pressure, and the adiabatic relation $T \sim n^{-1}$ with $\gamma$ $=5 / 3$ ) between density and temperature along the flow. From this very simple calculation, one would conclude that $a_{i} / \delta \sim t^{-1 / 2}$ along the flow. That is, the ion gyroradius apparently becomes smaller in relation to the boundary-layer width as the flow progresses downstream, as $t$ increases. A more careful calculation, however, shows that this conclusion is too hasty.

The above argument is deficient in that the initial plasmafield mixing-layer width in the breech $\delta_{b}$ was ignored. Also, there is no indication of a natural time scale for $t$. Both deficiencies can easily be corrected by allowing for the initial condition in the diffusive-broadening algorithm. (A more generalized plasma-field interface thickness calculation at the throat is performed in Appendix J. Also included is an example calculation of the fraction of attached plasma.) The algorithm now reads $\delta^{2}=\delta_{b}^{2}+D_{\mathrm{cl}} t$. Here, $\delta_{b}$ represents the initial ion penetration depth into the magnetic field. Carrying out the same derivation as in the above paragraph, one then finds that 


$$
\frac{a_{i}}{\delta}=\frac{1}{2} \beta_{i}^{1 / 2} \sqrt{\frac{n_{b} / n}{1+\frac{n_{b}}{n} \frac{t}{t_{b}}}}
$$

where the time scale $t_{b}$ is defined in terms of parameter values in the breech, $t_{b}=\delta_{b}^{2} / D_{b}$. Also, we have invoked the relation between ion gyroradius $a_{i}$ and ion skin depth $c / \omega_{p}$, in the form $\left(a_{i} / \delta\right)_{b}=0.5 \sqrt{\beta_{i}}$ for $\delta_{b}=2\left(c / \omega_{p} i\right)_{b}$. Now, relevant conditions are such that as the nozzle's throat is approached, $n_{b} / n \approx 1.5$ and $t<t_{b}$. The characteristic time $t_{b}$ is several hundred microseconds, for both classical and anomalous resistivity; whereas the time for longitudinal flow is only about $10 \mu \mathrm{s}$. The time $t_{b}$ scales as $T^{3 / 2} / n$. We infer that $a_{i} / \delta$ is practically unchanged between breech and throat, when the interface broadens because of the action of classical resistivity.

Next, the same argument is carried out with an anomalous resistivity, $\eta_{a}$, that arises from LHD microturbulence. The expression given below for this resistivity is derived later in this report. It applies to the case where the plasma-field interface width extends over several ion gyroradii. This resistivity is expected to dominate over classical resistivity at sufficiently high temperatures (see Sec. 3.5, "Comparison of Anomalous and Classical Resistivities"). One then has

$$
\begin{array}{r}
\eta_{a} \approx C_{\mathrm{Brack}}\left(\frac{\omega_{c e}^{2}}{\omega_{p e}^{2}}\right)\left(\frac{m_{e}}{m_{i}}\right)\left(\frac{1}{\omega_{L H}}\right)\left(\frac{V_{d}}{V_{\mathrm{th} i}}\right)^{2} \\
\text { where } 0.1 \leq \mathrm{C}_{\mathrm{Brack}} \leq 0.4
\end{array}
$$

The constant contour factor $C_{\text {Brack }}$ is not precisely known, but spans $0.1<C_{\text {Brack }}<0.4$. These values are based on a numerically obtained expression for anomalous resistivity in the Brackbill et al. computer simulations (Ref. 14) of the resistivity in a magnetoplasma gradient confined by magnetic pressure. Here, $\omega_{c e}$ is the electron gyrofrequency, $\omega_{p e}$ is the electron plasma frequency, and $\omega_{L H}=\sqrt{\omega_{c e} \omega_{c i}}$ is the lower hybrid gyrofrequency in the case of usual interest where $\omega_{p e}^{2} \gg \omega_{c e}^{2}$. In the above expression for anomalous resistivity $\eta_{a}$, in accordance with the notation introduced later in Section 3.2, "Linear Theory of Lower Hybrid Drift (LHD) Instability," $V_{d}$ represents the azimuthal drift velocity of electrons in the equilibrium current layer that separates plasma from the confining magnetic field. This notation is meant to apply in a reference frame in which the ions have no azimuthal drift velocity. Also, $V_{t h} i$ represents the ion thermal velocity, $\left(2 T_{i} / m_{i}\right)^{1 / 2}$.The anomalous resistive diffusivity in cgs units, $D_{a}=\left(c^{2} / 4 \pi\right) \eta_{a}$, therefore scales along the flow as

$$
\begin{aligned}
\frac{D_{a}}{\left(V_{d} / V_{\mathrm{th} i}\right)^{2}} \sim\left(\frac{B^{2}}{n}\right) \frac{1}{B} \sim \frac{\sqrt{n T}}{n} \sim \sqrt{\frac{T}{n}} & \\
& \sim \sqrt{\frac{n^{2 / 3}}{n}} \sim \frac{1}{n^{1 / 6}}
\end{aligned}
$$

Again, we have invoked radial pressure balance for $B^{2}$ and the adiabatic relation between $n$ and $T$ along the flow. It is important to note that the anomalous resistive diffusivity $D_{a}$ scales with the square of the current-drift velocity.

At first the initial plasma-field mixing-layer width as well as the factor $\left(V_{d} / V_{\text {th } i}\right)^{2}$ are neglected. Then, using the simple algorithm for variation of the boundary-layer width along the flow, $\delta^{2} \sim D_{a} t$, we can estimate the variation along the flow of the squared ratio of $a_{i}$ to $\delta$ (where $V_{\text {th } i}$ is the ion thermal velocity):

$$
\frac{a_{i}^{2}}{\delta^{2}} \sim\left(\frac{V_{t h i}^{2}}{\omega_{c i}^{2}}\right) \frac{1}{D_{a} t} \sim\left(\frac{T}{B^{2}}\right) \frac{n^{1 / 6}}{t} \sim\left(\frac{T}{n T}\right) \frac{n^{1 / 6}}{t} \sim \frac{1}{n^{5 / 6} t}
$$

The ratio itself then would vary along the flow as

$$
\frac{a_{i}}{\delta} \sim \frac{1}{n^{5 / 12} t^{1 / 2}}
$$

For the reasons mentioned earlier, however, it would be too hasty to accept this result for the variation of $a_{i} / \delta$ along the flow. Instead, taking into account the initial layer thickness as in the earlier calculation with classical resistivity, and now also including the factor $\left(V_{d} / V_{\text {th } i}\right)^{2}=\left(a_{i} / \delta\right)^{2}$ in the anomalous resistive diffusivity $D_{a}$, we find

$$
\frac{a_{i}}{\delta}=\frac{1}{2} \beta_{i}^{1 / 2} \sqrt{\frac{n_{b} / n}{1+\left(\frac{n_{b}}{n}\right)^{1 / 6} \frac{\left(a_{i} / \delta\right)^{2}}{\beta_{i} / 4}\left(\frac{t}{t_{b}}\right)}}
$$

Although the ratio of interest still appears on the right-hand side in the denominator, that is immaterial in the parameter range of interest here because the transit time $t_{b t}$ is small: $t_{b t} \ll<t_{b}$. As before, the characteristic time $t_{b}$ is defined by $t_{b}=\delta_{b}{ }^{2} /\left(D_{a}\right)_{b}$. The time $t_{b}$ scales as $(n T)^{-1 / 2}$. Also as before, $n_{b} / n \approx 1.5$ between breech and throat. 
Thus, we again infer that the ratio of the ion gyroradius to the interface layer width is practically unchanged between breech and throat. In the breech, this ratio is estimated to be about $1 / 3$. Hence our review of the LHD instability and its nonlinear evolution will be performed within the context of a plasma-field interface width that extends over several ion gyroradii, at any point along the flow between breech and throat. The overall conclusion is that the emphasis on the anomalous LHD resistivity should be in the small-iongyroradius regime $\left(a_{i}<\delta\right)$.

Avoiding the time integral of resistive diffusivity $D$ (whether classical or anomalous) really means that the average value of $D$ between breech and throat has been used. This method makes sense here because the plasma parameters do not vary much between breech and throat. The density at the throat drops to about two-thirds of its breech value, and the throat temperature drops to three-fourths of its breech value.

A final remark is that further downstream beyond the throat of the nozzle, there is a rapid spatial drop in plasma temperature with increased coulomb scattering of electrons. One would then expect that beyond the throat, classical resistivity (which is almost independent of density) would become more important than the LHD-based resistivity. The latter is basically independent of temperature and almost independent of plasma density along the flow (see the above scaling of $D_{a}$ ). Thus, the discussion of microturbulent LHD resistivity $\eta_{a}$ will be set within the context of edge plasma that is situated between the breech and the throat, where $\eta_{a}$ is most likely to matter.

\subsection{Linear Theory of Lower Hybrid Drift (LHD) Instability}

This subsection briefly outlines the linear theory of the small amplitude phase of the azimuthal mode LHD instability and summarizes the principal results for the frequency, growth rate, and wave number of this mode. These results then are utilized in the following Subsection 3.3, "QuasiLinear Evolution of LHD Instability and Anomalous Resistivity" on the nonlinear evolution of the mode and the development of an anomalous resistivity due to the mode. It is understood that all calculations are being done in the local longitudinal reference frame of the edge plasma. Along the way, we point out the limitations of the simplified models that are often utilized and the results of certain papers that attempted to overcome these limitations. There are three principal limitations, which can be described as follows:

(1) The Local Approximation: Most of the papers dealing with the linear theory have assumed that the magnetic field is in the $\mathrm{z}$-direction and that the wave propagates in the azimuthal $(y-)$ direction, with the mode structure $\mathrm{e}^{[i(k y-\omega t)]}$.
This is then of the "flute-mode" type. The mode frequency $\omega$ may be complex, signifying temporal mode growth. Notably, the radial structure of the mode, along the plasma density gradient in the $\mathrm{x}$-direction, is neglected. Such neglect of the radial structure of the mode is called the local approximation.

(2) The Electrostatic Approximation: It is commonly assumed that the charged particle density fluctuations both produce and respond only to fluctuations in the electric field. Fluctuations in the confining magnetic field are ignored. This is known as "the electrostatic approximation." Therefore, the fluctuating electric field $\{\delta \mathbf{E}\}$ is in the azimuthal $\mathbf{y}$-direction, along the azimuthal wave vector $\mathbf{k}$; the electric field fluctuation then is called longitudinal.

(3) The Unmagnetized Ion Approximation: It is assumed that the important frequencies and growth rates are so large, and the mode wavelengths are so short, that the effect of the direct current $(\mathrm{dc})$ magnetic field on the ion orbits can be ignored. That is, during a mode-fluctuation time, the ions cannot nearly complete a gyro-orbit. This is called the unmagnetized ion approximation. Therefore, the unperturbed ion orbits, which appear in the linear theory, are just straightline orbits.

Attempts to relax these three restrictions will be summarized at the end of this subsection. With these three restrictions in force, the dispersion relation for the mode is derived as follows. One starts with one of the Maxwell equations, Ampere's law in the electrostatic approximation, in which the fluctuating magnetic field $\{\delta \mathbf{B}\}$ is ignored (we shall use cgs units here, with $c$ being the speed of light):

$$
\nabla \times\{\delta \mathbf{B}\}=\left(\frac{1}{c}\right) \partial_{t}\{\delta \mathbf{D}\}=0
$$

Instead of setting $\nabla \times\{\delta \mathbf{B}\}=0$, the desired result could be achieved just by taking the divergence of Ampere's law. The vector $\{\delta \mathbf{D}\}$ is the electric displacement vector. In terms of Fourier-mode amplitudes, this equation becomes

$$
\left(\frac{-i \omega}{c}\right) D_{y}^{(1)}(k, \omega)=\left(\frac{-i \omega}{c}\right) \varepsilon(k, \omega) E_{y}^{(1)}(k, \omega)=0
$$

The superscript "(1)" means that the Fourier-mode amplitude corresponds to a first-order small perturbation. The $\{\delta \mathbf{D}\}$ is analyzed into its Fourier amplitudes $D_{y}^{(1)}(k, \omega)$, and the fluctuating electric field $\{\boldsymbol{\delta E}\}$ is analyzed into its Fourier components $E_{y}^{(1)}(k, \omega)$. We have used the fact that the electric displacement component $D_{y}^{(1)}(k, \omega)$ is related to the electric field component $E_{y}^{(1)}(k, \omega)$ through the dielectric function $\varepsilon(k, \omega)$. 
In order that the mode amplitude in Equation (134) be nonzero, the dielectric function must vanish:

$$
\varepsilon(k, \omega)=0
$$

This equation constitutes the dispersion relation that determines the real part of the mode's frequency Real $(\omega)$ and growth rate, which is the imaginary part, $\operatorname{Imaginary}(\omega)=\gamma$, in terms of the azimuthal mode wave number $k$. It is found that the growth rate of the LHD mode is maximized at a characteristic value of $k$, which is near the reciprocal of the electron thermal gyroradius $1 / a_{e}$. The specific form of the dielectric function is to be obtained from the linearized small-amplitude dynamics of the electrons and the ions.

It is convenient to express Equation (135) in terms of the electric susceptibilities of each charge species, $\chi_{e}(k, \omega)$ and $\chi_{i}(k, \omega)$. Then Equation (135) reads

$$
1+\chi_{e}(k, \omega)+\chi_{i}(k, \omega)=0
$$

The $\chi_{e}$ and $\chi_{i}$ can be formally obtained from the particle species conservation equations and the relation of species current densities to the fluctuating electric field. Ultimately, these formal relations must be given substance by means of the Vlasov dynamical equations for the velocity distribution functions of each charge species; this shall be done in the next Subsection 3.3, but only for $\chi_{i}(k, \omega)$. It will be seen that, because of Equation (136), only $\chi_{i}$ needs to be used to obtain the anomalous resistivity in the quasi-linear version of the nonlinear evolution of the mode.

Of course $\chi_{e}$ also must be known to obtain the dispersion relation Equation (136) in the linear theory. In the unmagnetized ion model $\chi_{e}$ is much more complicated than $\chi_{i}$ because the electrons sense the dc magnetic field while the ions do not. Therefore $\chi_{e}$ will not be derived here. The detailed expression for $\chi_{e}$ may be found in the paper of Davidson and Gladd (Ref. 18). Reference 18 also contains background references to the LHD instability.

The electric susceptibilities, $\chi_{e}$ and $\chi_{i}$, are now related to the species polarizabilities, $\alpha_{e}$ and $\alpha_{i}$, in order to indicate how the Vlasov equations for the species dynamics enter the picture. From the continuity equation for each charge species " $s$,"

$$
\partial_{t}\left\{\delta n_{s}\right\}+\nabla \cdot \frac{\left\{\delta \mathbf{J}_{s}\right\}}{q_{s}}=0
$$

Here, $\left\{\delta n_{s}\right\}$ is the number density fluctuation of a species, $\left\{\delta \mathrm{J}_{s}\right\}$ is the current density fluctuation of the species, and the particle charge of the species is $q$ ( $q=q$ for ions and $q=-q$ for electrons). The negative charge of the electron will be explicitly indicated $-q_{e}$; thus $q_{e}$ itself is a positive quantity. In terms of Fourier amplitudes, the above charge conservation equation can be written as

$$
-i \omega n_{s}^{(1)}(k, \omega)+\frac{i k J_{y s}^{(1)}(k, \omega)}{q_{s}}=0
$$

but the Fourier amplitude of the current density fluctuation is related to the Fourier amplitude of the electric field fluctuation by the electrical conductivity $\sigma_{s}$ for that species, namely $J_{y s}^{(1)}(k, \omega)=\sigma_{s}(k, \omega) E_{y}^{(1)}(k, \omega)$. The charge-density Fourier amplitude of each species is then given by

$$
n_{s}^{(1)}(k, \omega) q_{s}=\left(\frac{k}{\omega}\right) \sigma_{s} E_{y}^{(1)}(k, \omega)=\alpha_{s}(k, \omega) E_{y}^{(1)}(k, \omega)
$$

where $\alpha_{s}=(k / \omega) \sigma_{s}$ is the polarizability of species "s." That is, polarizability is the ratio of the charge density fluctuation of species " $s$ " to the electric field fluctuation. This ratio can be calculated by solving the Vlasov equation, as will be carried out for ions in the next subsection.

The Vlasov equation (the collisionless Boltzmann equation), is merely a statement of conservation of particles in position-velocity phase space.

We can now return to Ampere's law expressed in terms of explicit currents and electric fields, instead of using the electric displacement vector. This allows the derivation of another equivalent form of Equation (136). Ampere's law in the electrostatic approximation is

$$
\nabla \times\{\delta \mathbf{B}\}=0=\left(\frac{4 \pi}{c}\right)\{\delta \mathbf{J}\}+\left(\frac{1}{c}\right) \partial_{t}\{\delta \mathbf{E}\}
$$

The divergence of this equation, even keeping $\nabla \times\{\delta \mathbf{B}\}$, reduces to Poisson's equation relating the divergence of the electric field to charge density when charge conservation is invoked. It is actually unnecessary to set $\nabla \times\{\delta \mathbf{B}\}=0$, provided that only the electrostatic fluctuations are allowed to have an effect within the model.

The equation for the Fourier amplitudes then reads

$0=\left(\frac{4 \pi}{c}\right) J_{y e}^{(1)}(k, \omega)+\left(\frac{4 \pi}{c}\right) J_{y i}^{(1)}(k, \omega)-\left(\frac{i \omega}{c}\right) E_{y}^{(1)}(k, \omega)$

Use of the conductivity relation $J_{y S}^{(1)}(k, \omega)=\sigma_{s}(k, \omega) E_{y}^{(1)}(k, \omega)$, and multiplication by $i k / \omega$ and then dividing by $k$ yields

$$
\begin{aligned}
0=1+\left(\frac{4 \pi i}{k}\right)\left(\frac{\sigma_{e} k}{\omega}\right)+\left(\frac{4 \pi i}{k}\right) & \left(\frac{\sigma_{i} k}{\omega}\right) \\
& =1+\left(\frac{4 \pi i}{k}\right)\left(\alpha_{e}+\alpha_{i}\right)
\end{aligned}
$$


Comparing Equations (142) and (136), the susceptibility is related to the polarizability of species " $s$ " by

$$
\chi_{s}=\left(\frac{4 \pi i}{k}\right) \alpha_{s}
$$

recalling that $\alpha_{s}$ is the ratio of the charge density fluctuation of the mode to the electric field fluctuation in the mode. Use in Equation (143) of the expressions for $\alpha_{s}(s=e, i)$ obtained from the Vlasov equation, and then use of $\chi_{s}$ in Equation (136), yields the specific dispersion relation. This dispersion relation provides values of complex $\omega$ for given azimuthal wave number $k$.

The results of this dispersion relation must be obtained numerically and presented graphically, as is done in the paper of Davidson and Gladd (Ref. 18). All three of the approximations mentioned above were invoked in Reference 18. However, analytical results for growth rate maximized over $k$ do become available when $T_{e} \ll T_{i}$. These results of the linear theory are summarized below, and are utilized in the nonlinear (quasi-linear) theory in the next subsection. Moreover, it conveniently turns out that the numerical results are not greatly changed when $T_{e}=T_{i}$, as is apparent from the numerical results in Figures 2 and 3 in the paper of Davidson and Gladd (see also Refs. 19 and 22). Thus, the results presented here are not greatly sensitive to the electron-ion temperature ratio.

At the wave number for which the LHD-mode growth rate $\gamma$ is maximized, and when $T_{e} \ll T_{i}$, one has

$$
\begin{gathered}
\gamma=\frac{\sqrt{2 \pi}}{8}\left(\frac{V_{d}}{V_{\mathrm{th} i}}\right)^{2} \omega_{L H} \\
k a_{e}=\sqrt{\frac{2 T_{e}}{T_{i}}} \text { or also } k V_{\mathrm{th} i}=\sqrt{2} \omega_{L H} \\
\operatorname{Real}(\omega)=\left(\frac{1}{\sqrt{2}}\right)\left(\frac{V_{d}}{V_{\mathrm{th} i}}\right) \omega_{L H}=\frac{1}{2} k V_{d}
\end{gathered}
$$

Here, $V_{d}$ is the azimuthal current drift velocity in the macroscopic rest frame of the ions, $V_{\mathrm{th} i}$ is the ion thermal velocity $\sqrt{2 T_{i} / m_{i}}$, and $a_{e}$ is the thermal electron gyroradius. Also, $\omega_{L H}$ is the lower hybrid gyrofrequency $\sqrt{\omega_{c e} \omega_{c i}}$. The results in Equation (144) were obtained in the regime $V_{d}<V_{\text {th } i}$. This proves to be the regime of interest, based on the earlier calculations of the ratio $a_{i} / \delta<1$, presented at the end of the subsection on the initial width of the plasma-field mixing layer.
Note that in this regime the LHD mode is destabilized $(\gamma>0)$ at arbitrarily small current drift velocities $V_{d}$. This small drift-velocity condition corresponds to arbitrarily broad density gradients. That is because when $T_{e} \ll T_{i}$, the drift-to-ion thermal velocity ratio becomes $V_{d} / V_{\text {th } i}=0.5 a_{i} / x_{n}$, where $a_{i}$ is the thermal ion gyroradius and $1 / x_{n}=\left|n^{-1} \partial_{x} n\right|$ is the reciprocal gradient length $\left(x_{n}\right.$ is the quasi-radial length of decay of density). However, the apparent ease with which the mode is destabilized proves to be an artifact of the unmagnetized ion approximation, as shown in Reference 19. Nevertheless, within the context of plasma propellant flow in the magnetic nozzle, Equation (144a) yields an effective cutoff at which the mode loses importance for the production of microturbulent resistivity.

To make such an estimate relevant to space-vehicle propulsion, we note the following parameter values. A hydrogen ion gyroperiod would be of order $0.1 \mu \mathrm{s}$, whereas a characteristic longitudinal transit time for propellant to traverse a $1-\mathrm{m}$ nozzle would be of order $10 \mu \mathrm{s}$. On that basis, a growth rate smaller than about $0.01 \omega_{c i}$ should be ignored for practical purposes.

For hydrogen plasma propellant with $T_{e}<T_{i}$, the effective limiting width of the plasma-field mixing layer is estimated from Equation (144a) to be about 20 ion gyroradii, which actually proves to be in good semiquantitative agreement with the Freidberg-Gerwin cutoff (Ref. 19). For $T_{e}=T_{i}$, Reference 19 would predict about 12 ion gyroradii in hydrogen plasma. This degree of agreement comes from the circumstance that, in the solutions of the LHD dispersion relation allowing for magnetized ion gyroresonances, the growth-rate envelope of the individual ion gyroresonances is in good agreement with the theory having unmagnetized ions. Unfortunately, this condition for effective LHD-mode stabilization would imply at least about $10 \mathrm{~cm}$ of radius having plasma attached to magnetic flux. It should be remembered that this result is burdened with the three approximations mentioned earlier.

We end this subsection with an account of attempts to relax the three modeling restrictions. Gladd, Sgro, and Hewett investigated the local approximation both analytically and with computer simulation in Reference 20 . The simulation was based upon a so-called hybrid model using particle ions and fluid electrons. Self-consistently treating the radial structure of the eigenmodes in a nonlocal model, they found that, while not perfect, the local approximation still provided fairly accurate quantitative results. These are graphically compared with the exact results. While their model was comprehensive, their investigation had only a limited scope and left untreated other important aspects of edge-plasma stability (such as $\beta$ effects and gradient-width effects) that are relevant to the magnetic nozzle application. 
Drake, Huba, and Gladd addressed the accuracy of the electrostatic approximation for the LHD instability within a full electromagnetic treatment in Reference 15. In a linear theory, they found that gradually increasing local $\beta$ had the effect of relegating the LHD instability into the ion-cyclotron realm. For a local $\beta$ value below about 1.5 , the growth rate of the mode is still somewhat larger than $0.01 \omega_{c i}$, and so it would still have some relevance to the space-propulsion applications of the magnetic nozzle considered in this report. As the local $\beta$ is further increased up to 2.0 , however, the growth rate suddenly takes a steep dive down towards very small values that have absolutely no relevance. These results from a full electromagnetic treatment are important for magnetic nozzle applications because in the plasma-field mixing layer the local $\beta$ ranges from very small out in the magnetic field region to very large in the plasma region.

A few remarks are in order here, to provide a perspective on the finite- $\beta$ results of Reference 15 . First, the finite- $\beta$ mechanism that reduces the growth rate of the mode is the absorption of wave energy by those electrons resonant with the wave in virtue of their grad- $B$ guiding-center drift. This means that those particular electrons can be knocked out of resonance by collisions, and the wave can then be collisionally destabilized. This possibility deserves further examination within the context of the magnetic nozzle application. Second, Reference 15 utilized the local approximation. However, an understanding of the edge plasma in the magnetic nozzle requires a nonlocal treatment of the radial mode structure all across the edge plasma that also takes into account the presence of a large radial variation of $\beta$. Third, the calculation in Reference 15 was not self-consistent because the ions were modeled as unmagnetized even though the LHD mode was degraded into the ion-cyclotron regime by $\beta$ exceeding 1 . Thus, it seems that there is still some relevant work to be done in the area of linear theoryoriented to the magnetic nozzle application - that includes electromagnetic effects, collisions of electrons with ions, nonlocal mode structure, and ion orbits that sense the ambient magnetic field. E.Y. Choueiri (Ref. 21) studied a type of instability closely related to the LHD but that has a component of wave vector along the magnetic field. This is called the Modified Two-Stream instability in its lowtemperature form, and it is called the Electron Acoustic instability (in Soviet journals) in its high-temperature form. In his thesis, the instability is modeled as drift driven by external electric fields without the presence of plasma gradients. However, in his development of the theory for application to coaxial plasma thrusters, Choueiri included electromagnetic effects (hence non-zero $\beta$ ) and electron collisions. His treatment is analogous to the local approximation of the LHD instability and also is limited to unmagnetized ions.

Finally, Freidberg and Gerwin (Ref. 19) addressed the unmagnetized ion approximation. By taking into account the effect of the ambient magnetic field on the ion orbits, it was found that there is a continuous transformation of the LHD instability into the Ion Cyclotron Drift instability. This transformation occurs as the ratio $V_{d} / V_{\mathrm{th} i}=0.5\left(1+T_{e} / T_{i}\right) a_{i} / x_{n}$ decreases, hence as the gradient broadens. The absence of an instability cutoff at low drift velocities, found in the unmagnetized ion approximation, is thereby corrected. For deuterium plasma, the instability is stabilized when the gradient width exceeds $17 a_{i}$, and it is stabilized at about $12 a_{i}$ for hydrogen plasma, which implies, as earlier, an undesirably large fraction of plasma attached to magnetic nozzle flux. A note of caution is that Reference 19 still relied on both the local approximation and the electrostatic approximation. Hence, as mentioned above, a comprehensive linear theory free of approximations and oriented to the magnetic nozzle application would still be useful.

Huba and Ossakow (Ref. 22) clarified the role of collisions in the LHD instability, especially as regards its transition into the Ion Cyclotron Drift instability at low drift velocities. They showed that even a very small amount of ion collisionality, $m_{e} / m_{i}<v_{i} / \omega_{c i}<\sqrt{m_{e} / m_{i}}$, destroyed the ion-cyclotron resonance features. The parameters of interest lie near the low-collisionality end of the indicated range. The ion-resonance features become smoothed out by occasional ion collisions into behaviors that make the instability appear as if it were the LHD instability with unmagnetized ions. In this manner, the LHD instability acquires more significance than previously anticipated, within the low-drift regime. It should be emphasized that the linearized model of Huba and Ossakow is electrostatic, hence strictly valid only for $\beta=0$. One can speculate that if the same results proved to be true for the electromagnetic case, then the finite- $\beta$, linearized theory of Drake, Huba, and Gladd (Ref. 15), with unmagnetized ions, would also accrue more validity.

\subsection{Quasi-Linear Evolution of LHD Instability and Anomalous Resistivity}

Several nonlinear hypotheses have been advanced for the saturation level of microturbulent fluctuations arising from the LHD instability in magnetoplasma gradients. The saturation level of those fluctuations, in turn, determines the magnitude and parameter dependence of the anomalous resistivity $\eta_{a}$ within those gradients. The various saturation hypotheses have been enumerated, with references, in the paper of Gladd, Sgro, and Hewett (Ref. 20), and in Brackbill et al. (Ref. 14). Those hypotheses will be briefly summarized in the subsection following this one, 3.4 "Alternative Models for Saturation of LHD Microturbulence."

The main purpose of this subsection, however, is to review the second-order, single-mode, quasi-linear hypothesis, as the development of this particular model is easy to understand. Moreover, an expression is thereby arrived at for $\eta_{a}$, which - surprisingly - is essentially the same as the results of other models, as well as those of some simulations. 
It is understood that all calculations are being done in the local longitudinal reference frame of the edge plasma. The result of the quasi-linear model for $\eta_{a}$, which is in essential agreement with an electromagnetic simulation described in Reference 14 except for the $\beta$ dependence, will be compared with classical resistivity. (There is a difficulty of interpreting the simulation in (Ref. 14) with regard to the $\beta$ dependence of $\eta_{a}$, which is provisionally obviated by simply taking $\beta$ of order 1 in the plasma-field mixing layer.)

We begin with the Vlasov equation for the electron component of plasma. The effect of the ambient static magnetic field on the electron orbits must be taken into account. (In the electrostatic approximation, the electrons do not respond to fluctuations in the magnetic field.) Coulomb collisions of electrons are neglected for simplicity. This equation for the electron distribution function $\mathrm{f}_{e}\left(\mathbf{r}_{e}, \mathbf{v}_{e}, t\right)$ reads as follows:

$$
\partial_{t} \mathrm{f}_{e}+\nabla_{\mathbf{H}} \cdot\left[\left.\left(\frac{\mathrm{dH}}{\mathrm{d} t}\right) \mathrm{f}_{e}\right|_{\rfloor}=0\right.
$$

Here, the state of a particle is represented by a point in sixdimensional phase space $\mathbf{H}$, symbolized as follows by the set of mutually independent position and velocity variables:

$$
\mathbf{H}=\left(\mathbf{r}_{e}, \mathbf{v}_{e}\right)
$$

In Equation (145), the formal time-rate-of-change of those variables, as if along an orbit (although not), is defined here by

$$
\frac{\mathrm{d} H}{\mathrm{~d} t}=\left(\mathbf{v}_{e}, \mathbf{a}_{e}\right)
$$

The acceleration $\mathbf{a}_{e}$ of an electron particle is given in terms of electric field $\mathbf{E}$ and magnetic field $\mathbf{B}$, in cgs units, by

$$
\mathbf{a}_{e}=\left(\frac{-q}{m_{e}}\right)\left(\mathbf{E}+\frac{\mathbf{v}_{e} \times \mathbf{B}}{c}\right)
$$

Note that electric and magnetic fields $\mathbf{E}$ and $\mathbf{B}$ in Equation (148) depend on particle position $\mathbf{r}_{e}$ but not on particle velocity $\mathbf{v}_{e}$. In Equation (145), $\mathbf{r}_{e}$ and $\mathbf{v}_{e}$ constitute mutually independent variables in phase space, and they also are independent of the time variable $t$.

For purposes of the particle-continuity interpretation of Equation (145), which involves interpretation of the phasespace divergence operator, $\mathbf{v}_{e}$ in the first component of Equation (147) is considered to be a vector directed within $r$-space, and $\mathbf{a}_{e}$ in the second component is considered to be directed within $v$-space. Accordingly, the phase-space divergence operator in Equation (145), namely $\nabla_{\mathbf{H}} \cdot\left[(\mathrm{d} \mathbf{H} / \mathrm{d} t) \mathrm{f}_{e}\right]=$ $\nabla_{\mathbf{r}} \cdot\left[(\mathrm{d} \mathbf{H} / \mathrm{d} t) \mathrm{f}_{e}\right]+\nabla_{\mathbf{v}} \cdot\left[(\mathrm{dH} / \mathrm{d} t) \mathrm{f}_{e}\right]$, is to be applied to the respective components of $\mathrm{dH} / \mathrm{d} t$, as defined in Equation (147). This procedure is described in detail below.

The distribution function $\mathrm{f}_{e}$, which is to be determined by the partial differential Equation (145), is the phase-space distribution function of the electrons, $\mathrm{f}_{e}(\mathbf{H}, t)$. This function is the number of electrons per unit phase volume at the point $\mathbf{H}=\left(\mathbf{r}_{e}, \mathbf{v}_{e}\right)$ at time $t$. That is, $\mathrm{f}_{e}(\mathbf{H}, t)$ is the number density of electron particles in phase space. The flux of electrons through phase space is just $\left[(\mathrm{d} \mathbf{H} / \mathrm{d} t) \mathrm{f}_{e}\right]$, the first component of which (see Eq. (147)) is the particle flux through ordinary space, and the second of which is regarded as the flux through "velocity" space.

Now consider a fixed element of phase volume, $\mathrm{d}^{3} r \mathrm{~d}^{3} v$, at phase point $\mathbf{H}$. Consistent with the above description of electron flux through phase space, the spatial divergence $\left[\nabla_{\mathbf{r}} \cdot\left(\mathbf{v}_{e} \mathrm{f}_{e}\right) \mathrm{d}^{3} r \mathrm{~d}^{3} v\right]$ signifies the rate at which electrons leave "ordinary-volume" element $\mathrm{d}^{3} r$; likewise, the velocity divergence $\left[\nabla_{\mathrm{v}} \cdot\left(\mathbf{a}_{e} \mathrm{f}_{e}\right) \mathrm{d}^{3} r \mathrm{~d}^{3} v\right]$ signifies the rate at which they leave "velocity-volume" element $\mathrm{d}^{3} v$. The sum of these rates constitutes the total rate of egress of electrons, $-\partial_{t} f_{e}$ $d^{3} r d^{3} v$, away from the combined volume element, $d^{3} r d^{3} v$. This rate balance is encapsulated in Equation (145), which thus signifies the local continuity of a gas of particles in phase space. Equation (145) closely resembles the equation of local mass continuity in ordinary fluid dynamics.

Since $\mathbf{r}_{e}$ and $\mathbf{v}_{e}$ are regarded as mutually independent variables, the first part of the divergence operator reads as

$$
\nabla_{\mathbf{r}} \cdot\left(\mathbf{v}_{e} \mathrm{f}_{e}\right)=\mathbf{v}_{e} \cdot \nabla_{\mathbf{r}} \mathrm{f}_{e}
$$

The second part of the divergence operator is

$$
\nabla_{\mathbf{v}} \cdot\left(\mathbf{a}_{e} \mathrm{f}_{e}\right)=\mathbf{a}_{e} \cdot \nabla_{\mathbf{v}} \mathrm{f}_{e}
$$

Equation (148) for the particle acceleration, $\mathbf{a}_{e}$, was utilized in Equation (149b). A detailed justification of Equation (149b) uses the fact that $\mathbf{E}$ and $\mathbf{B}$ in Equation (148) depend only upon $\mathbf{r}_{e}$ and not on $\mathbf{v}_{e}$; and also uses the fact that $\nabla_{\mathbf{v}} \cdot\left(\mathbf{v}_{e} \times \mathbf{B f}\right)=\mathbf{v}_{e} \times \mathbf{B} \cdot \nabla_{\mathbf{v}} \mathrm{f}_{e}+\mathrm{f}_{e} \nabla_{\mathbf{v}} \cdot\left(\mathbf{v}_{e} \times \mathbf{B}\right)=\mathbf{v}_{e} \times \mathbf{B} \cdot \nabla_{\mathbf{v}} \mathrm{f}_{e}+0$. 
Now using Equation (149) and $\nabla_{\mathbf{H}} \cdot\left[(\mathrm{dH} / \mathrm{d} t) \mathrm{f}_{e}\right]=\nabla_{\mathbf{r}} \cdot\left[(\mathrm{dH} / \mathrm{d} t) \mathrm{f}_{e}\right]$ $+\nabla_{\mathbf{v}} \cdot\left[(\mathrm{dH} / \mathrm{d} t) \mathrm{f}_{e}\right]$, the Vlasov Equation (145) for the electron distribution function can be written as follows:

$$
\begin{aligned}
\partial_{t} \mathrm{f}_{e}+\nabla_{\mathbf{H}} \cdot\left(\left[\frac{\mathrm{d} \mathbf{H}}{\mathrm{d} t}\right]_{]} \mathrm{f}_{e}\right) & =\partial_{t} \mathrm{f}_{e}+\mathbf{v}_{e} \cdot \nabla_{\mathbf{r}} \mathrm{f}_{e} \\
& +\frac{(-q)}{m_{e}}\left(\mathbf{E}+\frac{\mathbf{v}_{e} \times \mathbf{B}}{c}\right) \cdot \nabla_{\mathbf{v}} \mathrm{f}_{e}=\mathbf{0}
\end{aligned}
$$

Equation (150) is the conventional form of the Vlasov equation for the electron phase-space distribution function. Collisions of electrons with electrons and with ions are neglected. Replacing $-q / m_{e}$ by $q / m_{i}$, it becomes the Vlasov equation for the ion phase-space distribution function. We now present the quasi-linear formulation of the microturbulent resistivity of the magnetoplasma, called anomalous resistivity, as it cannot be described in terms of coulomb collisions of electrons with ions.

First, the anomalous resistivity of the plasma, $\eta_{a}$, will be formulated in terms of the electric-field fluctuation energy by taking the statistical ensemble average of Equation (150) averaged over a large number of systems, identical but for the indeterminate phase of the LHD wave. The indeterminate phase of the wave is due to the fact that there is no preferred point in azimuth from which the wave should originate, nor is there a preferred point in time at which the wave should suddenly appear. That is, the unperturbed configuration is uniform in the y-direction and uniform in its static behavior in time. The phase of the wave is here regarded as a random variable, which is present in the form of a complex exponential phase factor implicitly attached to the Fourier amplitudes associated with the wave.

Second, the saturation level of the electric field fluctuations, which determines $\eta_{a}$, will be estimated from energy balance as in the paper of Davidson and Gladd (Ref. 18). A very important refinement of that early energy balance argument then becomes necessary, as was later pointed out by Davidson (Ref. 23).

Ensemble-averaged quantities are represented by brackets: for example, $f=\langle f\rangle+\{\delta f\}$ and $n=\langle n\rangle+\{\delta n\}$. The ensemble average of the Vlasov Equation (150) can then be written as follows:

$$
\begin{aligned}
\partial_{t}\left\langle\mathrm{f}_{e}\right\rangle+\mathbf{v}_{e} \cdot \nabla_{\mathbf{r}}\left\langle\mathrm{f}_{e}\right\rangle & \\
& -\frac{q_{e}}{m_{e}}\left(\langle\mathbf{E}\rangle+\frac{\mathbf{v}_{e}}{c} \times\langle\mathbf{B}\rangle\right) \cdot \nabla_{\mathbf{v}}\left\langle\mathrm{f}_{e}\right\rangle \\
& =\frac{q_{e}}{m_{e}}\left\langle\{\delta \mathbf{E}\} \cdot \nabla_{\mathbf{v}}\left\{\delta \mathbf{f}_{e}\right\}\right\rangle
\end{aligned}
$$

In the electrostatic approximation, the magnetic fluctuation $\{\delta B\}$ has been ignored. Note that the ensemble-averaged distribution function $\langle\mathrm{f}\rangle$ depends upon the averaged product of the linear fluctuations on the right-hand side. We will now define $\langle n\rangle=$ "ensemble averaged number density," and $\left\langle\mathbf{v}_{e}\right\rangle=$ "ensemble-averaged electron velocity." Also $\left\langle P_{e}\right\rangle$ will represent the ensemble-averaged electron pressure.

Multiplying Equation (151) by $m_{e} \mathbf{v}_{e}$ and integrating over all velocity space, $\int d^{3} v_{e}$, that equation becomes

$$
\begin{aligned}
\partial_{t}\left(m_{e}\langle n\rangle\left\langle\mathbf{v}_{e}\right\rangle\right)+\nabla \cdot\left(m_{e}\langle n\rangle\left\langle\mathbf{v}_{e}\right\rangle\left\langle\mathbf{v}_{e}\right\rangle\right)+\nabla\left\langle P_{e}\right\rangle \\
=-\langle n\rangle q\left(\langle\mathbf{E}\rangle+\frac{\left\langle\mathbf{v}_{e}\right\rangle \times\langle\mathbf{B}\rangle}{c}\right)-q\langle\{\boldsymbol{\delta} n\}\{\delta \mathbf{E}\}\rangle
\end{aligned}
$$

The second term is the divergence of a symmetric tensor. We have used the velocity moments of the distribution function, namely $\langle n\rangle=\int\langle\mathrm{f}\rangle \mathrm{d}^{3} v,\langle n\rangle\left\langle\mathbf{v}_{e}\right\rangle=\int\langle\mathrm{f}\rangle \mathbf{v}_{e} \mathrm{~d}^{3} v$, and $\delta n_{e}=\int\left\{\delta \mathrm{f}_{e}\right\} \mathrm{d}^{3} v$. Note the ensemble average of $n_{e}$ is quasi-neutral. We have also used the moment that defines the electron pressure tensor; that is not written down. The pressure tensor is assumed here to be a scalar (neglect of electron viscosity). These moment integrals extend over all of velocity space.

It is important to realize that the quantities $\langle n\rangle$ and $\left\langle\mathbf{v}_{e}\right\rangle$ are not simply the macroscopic density and electron velocity of the static, perturbation-free configuration, but that they embody as well the additional average effects of the secondorder products of the fluctuations. The macroscopically smooth electron pressure $P_{e}$ also includes both thermal and fluctuation contributions that have been averaged over, but it turns out that these detailed considerations regarding the pressure do not complicate the following discussion.

We suppose that the macroscopic ensemble-averaged system only has radial gradients $\partial / \partial x$ perpendicular to the z-directed magnetic field, and that $\left\langle v_{e}\right\rangle$, the electron current velocity, is in the azimuthal (y-) direction. Then the y-component of Equation (152), which is the macroscopic $y$-momentum equation, reduces to the following:

$$
\partial_{t}\left(m_{e}\langle n\rangle\left\langle v_{y e}\right\rangle\right)+\langle n\rangle q\left\langle E_{y}\right\rangle=-q\left\langle\left\{\delta n_{e}\right\}\left\{\delta E_{y}\right\}\right\rangle
$$

Here, $\left\{\delta n_{e}\right\}$ is a real electron density fluctuation, which is related to the complex wave-Fourier amplitude $n_{e}^{(1)}(k, \omega)$ and its complex conjugate $n_{e}^{(1)^{*}}(k, \omega)$ as follows: 


$$
\left\{\delta n_{e}\right\}=\frac{1}{2}\left(n_{e}^{(1)}+n_{e}^{(1) *}\right)
$$

Likewise,

$$
\left\{\delta E_{y}\right\}=\frac{1}{2}\left(E_{y}^{(1)}+E_{y}^{(1)^{*}}\right)
$$

As mentioned earlier, a random phase factor $e^{i \Psi_{\text {ran }}}$ is implicitly assumed to be incorporated in the complex Fourier amplitudes $n_{e}^{(1)}(k, \omega)$ and $E_{y}^{(1)}(k, \omega)$ in Equation (154). Consequently, in the phase-averaged second-order product $\left\langle\left\{\delta n_{e}\right\}\left\{\delta E_{y}\right\}\right\rangle$ in Equation (153), only the cross terms $\left(n_{e}^{(1)} E_{y}^{(1)^{*}}+n_{e}^{(1)^{*}} E_{y}^{(1)}\right)$ survive the average because the factor $e^{i \Psi_{\text {ran }}}$ cancels. The remaining terms are $\left(n_{e}^{(1)} E_{y}^{(1)}+n_{e}^{(1)^{*}} E_{y}^{(1)^{*}}\right)$, which after averaging, produce the factor $\left\langle\cos \left(2 \psi_{\text {ran }}\right)\right\rangle=0$. This description of the averaged contribution to $\left\langle\left\{\delta n_{e}\right\}\left\{\delta E_{y}\right\}\right\rangle$ is correct even when allowing for the fact that the wave frequency $\omega=\operatorname{Real}(\omega)+i \gamma$ is a complex quantity.

Therefore, the right-hand side of Equation (153) can be written as follows:

$$
\begin{array}{r}
-q\left\langle\left\{\delta n_{e}\right\}\left\{\delta E_{y}\right\}\right\rangle=-q \frac{1}{4}\left(n_{e}^{(1)} E_{y}^{(1)^{*}}+n_{e}^{(1)^{*}} E_{y}^{(1)}\right) \\
=-q \frac{1}{2} \operatorname{Real}\left(n_{e}^{(1)} E_{y}^{(1)^{*}}\right)=\frac{1}{2} \operatorname{Real}\left(-q n_{e}^{(1)} E_{y}^{(1)^{*}}\right)
\end{array}
$$

However, from the equations for the electron polarizability $\alpha_{e}$ and electron susceptibility $\chi_{e}$, from Equations (139) and (143), respectively, $\quad-q_{e} n_{e}^{(1)}=\alpha_{e} E_{y}^{(1)}=(k / 4 \pi i) \chi_{e} E_{y}^{(1)}$. Therefore,

$$
\begin{aligned}
-q\left\langle\left\{\delta n_{e}\right\}\left\{\delta E_{y}\right\}\right\rangle & =\frac{1}{2} \operatorname{Real}\left[\left.\left(\frac{k}{4 \pi i}\right) \chi_{e} E_{y}^{(1)} E_{y}^{(1)^{*}}\right|_{\rfloor}\right. \\
& =\frac{1}{2}\left(\frac{k}{4 \pi}\right) \operatorname{Imaginary}\left(\chi_{e}\right) E_{y}^{(1)} E_{y}^{(1)^{*}}
\end{aligned}
$$

The averaged fluctuation energy density in the real electric field fluctuations may be calculated in terms of its complex Fourier amplitude in the same manner as described above. One easily finds $\left\langle\left\{\delta E_{y}\right\}^{2}\right\rangle=1 / 2 E_{y}^{(1)} E_{y}^{(1)^{*}}$. Then Equation (156) can be reexpressed in terms of the real electric field fluctuation energy density. Equation (156) becomes

$$
\begin{array}{r}
-q\left\langle\left\{\delta n_{e}\right\}\left\{\delta E_{y}\right\}\right\rangle=2 k \text { Imaginary }\left(\chi_{e}\right)\left\{\left.\frac{\left.\left\langle\left\{\delta E_{y}\right\}^{2}\right\rangle\right|_{i}}{8 \pi}\right|_{j}\right. \\
=2 k \text { Imaginary }\left(\chi_{e}\right) W_{\mathrm{f} 1}^{E}
\end{array}
$$

The averaged energy density in the fluctuating electric field has here been defined by $W_{\mathrm{fl}}^{E}=\left\{\left\langle\left\{\delta E_{y}\right\}^{2}\right\rangle / 8 \pi\right\}$.

Now, in the LHD fluctuations, the electron susceptibility $\chi_{e}(k, \omega)$ is a very complicated object because the electron's orbit senses the magnetic field. Fortunately, in the quasilinear theory, $\omega$ and $k$ of a mode are still regarded as being related by the dispersion relation. It is expressed in Equation (136), which implies

$$
\operatorname{Imaginary}\left(\chi_{e}\right)=-\operatorname{Imaginary}\left(\chi_{i}\right)
$$

The ion susceptibility $\chi_{i}$ is easy to calculate when the ions are unmagnetized because of the short time scales (so ions make straight-line (degenerate) orbits only). Use of Equation (158) in Equation (157) and the latter in Equation (153) yields the macroscopic azimuthal electron momentum equation in the form

$$
\begin{aligned}
\partial_{t}\left(m_{e}\langle n\rangle\left\langle v_{y e}\right\rangle\right) & +\langle n\rangle q\left\langle E_{y}\right\rangle \\
& =-2 k \text { Imaginary }\left(\chi_{i}\right) W_{\mathrm{fl}}^{E}
\end{aligned}
$$

We will now calculate $\chi_{i}$ from the Vlasov equation for the ions.

In the macroscopic rest frame of the ions, neglecting the influence of the static ambient magnetic field as well as of magnetic fluctuations, the Vlasov equation for the ion phasespace distribution function $\mathrm{f}_{i}(\mathbf{r}, \mathbf{v}, t)$ reads

$$
\partial_{t} \mathrm{f}_{i}+\mathbf{v} \cdot \nabla_{\mathbf{r}} \mathrm{f}_{i}+\left(\frac{q \mathbf{E}}{m_{i}}\right) \cdot \nabla_{\mathbf{v}} \mathrm{f}_{i}=0
$$

In Equation (160), $\mathrm{f}_{i}(\mathbf{r}, \mathbf{v}, t)$ is the ion phase-space distribution function described earlier in this section. It represents the time-dependent number density of ions at position $\mathbf{r}_{i}$, and the time-dependent number density per velocity volume at velocity $\mathbf{v}_{i}$.

In the presence of plasma fluctuations, $f_{i}$ naturally splits into a time-independent part that is not directly dependent upon the fluctuations, and a part $\left\{\delta \mathrm{f}_{i}\right\}$ that directly participates in those fluctuations. Moreover, $\left\{\delta f_{i}\right\}$ can be Fourier analyzed (expanded in microscopic plane waves) as follows: 


$$
\left\{\delta f_{i}(\mathbf{r}, \mathbf{v}, t)\right\}=\sum_{\mathbf{k}} f_{i}^{(1)}(\mathbf{k}, \omega(\mathbf{k})) e^{i(\mathbf{k} \cdot \mathbf{r}-\omega(\mathbf{k}) t)}
$$

The quantity $f_{i}^{(1)}(\mathbf{k}, \omega(\mathbf{k}))$ is called the Fourier amplitude or the Fourier coefficient of $\left\{\delta f_{i}(\mathbf{k}, \omega(\mathbf{k}))\right\}$. In this Fourier expansion, the complex frequency $\omega(\mathbf{k})$ is related to the wave vector $\mathbf{k}$ via the dispersion relation. (The dispersion relation is the outcome of self-consistently applying the plasma dynamical equations together with Maxwell's electromagnetic equations.) In the present instance, we focus on those microscopic waves that possess only an azimuthal dependence (dependence on the $y$-coordinate).

The linearized equation for the fluctuations then reads

$$
\begin{aligned}
\partial_{t}\{\boldsymbol{\delta}\}_{i}+\mathbf{v} \cdot \nabla_{\mathbf{r}}\left\{\delta \mathrm{f}_{i}\right\}+ & \left(\frac{q \mathbf{E}_{\mathrm{eq}}}{m_{i}}\right) \cdot \nabla_{\mathbf{v}}\left\{\delta \mathrm{f}_{i}\right\} \\
& =-\left(\frac{q\{\delta \mathbf{E}\}}{m_{i}}\right) \cdot \nabla_{\mathbf{v}} f_{i}^{(0)}(k, \omega)
\end{aligned}
$$

In the macroscopic rest frame of the ions the ion pressure is electrostatically confined, so the equilibrium electric field in the quasi-radial (x-) direction is $E_{\text {eq }}=\left(T_{i} / q\right)\left(n^{-1} \partial_{x} n\right)$. Moreover, the third term in Equation (162) is on the order of $\left(q E_{\text {eq }} / m_{i} V_{\text {th }}\right)\left\{\delta f_{i}\right\}$ with $V_{\text {th } i}$ the ion thermal velocity, $V_{\text {th } i}=$ $\sqrt{2 T_{i} / m_{i}}$. The second term in Equation (162) is on the order of $\left(k V_{\text {th } i}\right)\left\{\delta f_{i}\right\}$ where $k$ is the mode wave number. Therefore, ratio of the third term to the second term proves to be $\sim 1 /\left(2 k x_{n}\right)$, where the gradient length is $x_{n}=1 /\left(n^{-1} \partial_{x} n\right)$. For the LHD modes of interest, $k a_{i}>1$, where $a_{i}$ is the thermal ion gyroradius. Actually, $k$ is rather larger than $1 / a_{i} ; k a_{e} \sim 1$. Therefore, $k x_{n} \gg 1$; and so the ratio of the third term to the second term in Equation (162), 1/(2kx $)$, is a very small number.

Accordingly, we neglect the third term in Equation (162) and also use the local approximation for an electrostatic mode with wave propagation in the azimuthal y-direction. Equation (162) then becomes

$$
\partial_{t}\left\{\delta f_{i}\right\}+v_{y i} \partial_{y}\left\{\delta f_{i}\right\}=-\left(\frac{q}{m_{i}}\right)\left\{\delta E_{y}\right\}\left(\frac{\partial f_{i}^{(0)}(k, \omega)}{\partial v_{y i}}\right)
$$

For a Fourier-mode $e^{(i k y-i \omega t)}$, this equation relates the Fourier amplitudes (corresponding to a first-order small perturbation) $f_{i}^{(1)}(k, \omega)$ and $E_{y}^{(1)}(k, \omega)$.

$$
-i \omega f_{i}^{(1)}+i k v_{y i} f_{i}^{(1)}=-\left(\frac{q}{m_{i}}\right) E_{y}^{(1)}\left(\frac{\partial f_{i}^{(0)}}{\partial v_{y i}}\right)
$$

The solution for the Fourier amplitude $f_{i}^{(1)}(k, \omega)$ of the ion's perturbed distribution function $\left\{\delta \mathrm{f}_{i}\right\}$ is then

$$
f_{i}^{(1)}(k, \omega)=i \frac{q}{m_{i}} \frac{E_{y}^{(1)}}{k} \frac{\partial f_{i}^{(0)} / \partial v_{y i}}{v_{y i}-\omega / k}
$$

Notice that for an unstable mode, $\omega$ has a positive imaginary part and therefore the denominator does not vanish. A nearresonant ion-particle response to the fluctuating fields arises when the unperturbed particle velocity $v_{y i}$ has a value such that the denominator in Equation (165) almost vanishes. Such resonances also can occur for the electrons, although the electron denominator is more complicated, including the electron gyrofrequency along with various electron guidingcenter drifts in the unperturbed orbits. The electron resonances can be nonlinearly broadened and are then thought to provide a mechanism of saturation of the microturbulence, as will be discussed below.

By calculating the Fourier amplitude of the ion number density, as given by

$$
n_{i}^{(1)}(k, \omega)=\int f_{i}^{(1)} \mathrm{d}^{3} v
$$

we can calculate the polarizability ratio $\alpha_{i}=q n_{i}^{(1)} / E_{y}^{(1)}$, and then the susceptibility $\chi_{i}=4 \pi i \alpha_{i} / k$ from Equation (143). The integration in Equation (166) extends over all of velocity space.

In carrying out the calculation indicated by Equations (165) and (166), we shall assume that the equilibrium distribution function of the ions is Maxwellian:

$$
f_{i}^{(0)}(k, \omega)\left(v_{i}\right)=n_{\mathrm{eq}}\left(\frac{V_{\mathrm{th} i}^{-2}}{\pi}\right)^{3 / 2} e^{-v_{i}^{2} / V_{\mathrm{th} i}^{2}}
$$

where $V_{\text {th } i}^{-2}=m_{i} / 2 T_{i}$. We have used and will be using $\sqrt{\pi}=\int_{-\infty}^{\infty} e^{-u^{2}} \mathrm{~d} u$. The constant in front of the gaussian exponential in Equation (167) is such that $\int f_{i}^{(0)}(k, \omega)(v) \mathrm{d}^{3} v=n_{\text {eq }}$. 
The calculation of the Fourier amplitude of the ion density fluctuation in Equation (166), from Equations (165) and (167), is uneventful and a bit tedious. The result only is written here:

$$
n_{i}^{(1)}(k, \omega)=-i \frac{q}{T_{i}} \frac{E_{y}^{(1)}}{k} \frac{n_{\mathrm{eq}}}{\sqrt{\pi}} \int_{-\infty}^{\infty} \frac{u e^{-u^{2}}}{u-V_{\mathrm{ph}}} \mathrm{d} u
$$

The complex normalized phase velocity is given by $V_{\mathrm{ph}}=(\omega / k) \sqrt{V_{\mathrm{th} i}^{-2}}$, where $V_{\text {th } i}^{-2}=m_{i} / 2 T_{i}$ with $V_{\text {th } i}$ being the ion thermal velocity. By subtracting and adding $V_{\mathrm{ph}}$ in the numerator of the integrand, the expression in Equation (168) can be put into a more convenient form:

$$
n_{i}^{(1)}(k, \omega)=-i \frac{q}{T_{i}} \frac{E_{y}^{(1)}}{k} n_{\mathrm{eq}}\left[1+\left(\frac{\omega}{k V_{\text {th } i}}\right) \mathrm{Z}\left(\frac{\omega}{k V_{\text {th } i}}\right)\right]
$$

where the so-called plasma dispersion function $Z(\zeta)$ is defined by

$$
Z(\zeta)=\frac{1}{\sqrt{\pi}} \int_{-\infty}^{\infty} \frac{e^{-u^{2}}}{u-\zeta} \mathrm{d} u
$$

in which $\zeta$ is taken to have a positive imaginary part. Note that $\zeta=\omega / k V_{\text {th } i}$ is the independent variable of $Z(\zeta)$ and that $V_{\text {th } i}$ in Equation (169) is the ion thermal velocity, $\sqrt{2 T_{i} / m_{i}}$.

The ratio $\alpha_{i}=q n_{i}^{(1)} / E_{y}^{(1)}$ is available from Equation (169). Then $\chi_{i}=4 \pi i \alpha_{i} / k$ can be written as follows:

$$
\chi_{i}=2 \frac{\omega_{p i}^{2}}{k^{2} V_{\text {th } i}^{2}}\left[1+\left(\frac{\omega}{k V_{\text {th } i}}\right) \mathrm{Z}\left(\frac{\omega}{k V_{\text {th } i}}\right)\right]
$$

The square of the ion plasma frequency has been introduced; thus, $\omega_{p i}^{2}=4 \pi n_{\mathrm{eq}} q^{2} / m_{i}$ in cgs units.

Returning now to the phase-averaged azimuthal momentum equation of the electrons (Eq. (159)), we suppose that there is a quasi-steady state relative to the rapid microscopic fluctuations. The reason for this is discussed below after Equation (174). Furthermore, we also set

$$
\left\langle E_{y}\right\rangle=\eta_{a}\left(-q\langle n\rangle\left\langle v_{y e}\right\rangle\right)=\eta_{a}\left\langle J_{y}\right\rangle
$$

which serves to define anomalous resistivity $\eta_{a}$. Then, from Equation (171), Equation (159) reduces to

$$
\begin{aligned}
\eta_{a}=4 k \frac{\omega_{p i}^{2}}{k^{2} V_{\mathrm{th} i}^{2}} \text { Imaginary }\left[\left(\frac{\omega}{k V_{\mathrm{th} i}}\right) \mathrm{Z}\left(\frac{\omega}{k V_{\mathrm{th} i}}\right)\right] \\
\times \frac{W_{\mathrm{fl}}^{E}}{q_{e}^{2}\left\langle n_{e}^{2} v_{y e}\right\rangle}
\end{aligned}
$$

Here, average electron velocity $\left\langle v_{y e}\right\rangle$ is identified with the azimuthal current drift velocity $V_{d}$ of the electrons, in the macroscopic rest frame of the ions. Also, recall that the second-order-small quantity $W_{\mathrm{fl}}^{E}$ is the average energy density in the fluctuating electric field. Thus, $W_{\mathrm{fl}}^{E}$ is a second-order-small factor in the expression for the anomalous resistivity. The second-order distinction between the equilibrium number density $n_{e q}$ in $\omega_{p i}^{2}$ in the numerator and the ensemble-averaged $\langle n\rangle$ in the denominator is then a correction of no consequence for the leading second-order evaluation of the right-hand side of Equation (173).

An anomalous collision frequency $v_{a}$ of electrons can be defined as usual, for singly ionized ions, by $\eta_{a}=m_{e} v_{a} /\langle n\rangle q^{2}$. We then find from Equation (173) that $v_{a} e$ is given by an expression that agrees exactly with Equation (33) in the paper of Davidson and Gladd (Ref. 18). Our derivation, however, differs somewhat from that in Reference 18.

Equation (173) is applied the to the small drift velocity regime, $V_{d} \ll<v_{i}$, which is equivalent to the case in which the gradient length $x_{n}$ is rather larger than a thermal ion gyroradius. This regime is consistent with our estimate of the initial width of the plasma-magnetic-field mixing layer. At the same time, we follow Reference 18 by using an energy argument to obtain an upper bound for the fluctuating electric-field energy density $W_{\mathrm{fl}}^{E}$. This procedure, with Equation (173), then yields an upper bound to the anomalous resistivity in the small-drift regime.

Referring to Equation (144) for properties of the most rapidly growing linear mode in the low drift velocity regime, the real frequency Real $(\omega)$ approaches zero with $V_{d}$, and the growth rate $\gamma$ approaches zero with $V_{d}^{2}$. The wave number at maximum growth, however, stays fixed as $V_{d} \rightarrow 0$. Thus, $\zeta=\omega / k V_{\text {th } i} \rightarrow 0$ as $V_{d} \rightarrow 0$. A simple contour integration then can be used to show that as $\omega \rightarrow 0$ from above the real axis, the Z-function in Equation (173) approaches the imaginary constant value, $i \sqrt{\pi}$. The imaginary quantity in Equation (173) Imaginary[ $\zeta Z(\zeta)$ ] can then be evaluated to leading order in the low-drift regime as 


$$
\text { Imaginary }\left[\left(\frac{\omega}{k V_{\text {th } i}}\right) \mathrm{Z}\left(\frac{\omega}{k V_{\text {th } i}}\right)\right]=\left[\frac{\operatorname{Real}(\omega)}{k V_{\text {th } i}}\right]_{]} \sqrt{\pi}
$$

In order to obtain an upper bound on anomalous resistivity $\eta_{a}$, it is noted by Davidson and Gladd (Ref. 18) (after Fowler) that the energy in the fluctuating electric field can only be supplied by the drift kinetic energy of the electron current (in the rest frame of the ions). This is thought by them to be the only source of energy available to be converted into fluctuation energy. We are supposing that this drift current is at least momentarily sustained by the local average azimuthal electric field, $\left\langle E_{y}\right\rangle$, which, in turn, is induced by the momentary local radial expansion of the plasma across the longitudinal magnetic nozzle field. Therefore, the electricfield fluctuation energy can at most be

$$
W_{\mathrm{fl}}^{E}=\frac{1}{2} m_{e}\langle n\rangle\left\langle v_{y e}\right\rangle^{2}
$$

Now, Equations (174) and (175), the expression for $\omega_{p i}^{2}$, as well as the properties of real frequency $\operatorname{Real}(\omega)$ and azimuthal wave number $k$ are used in Equation (144). Then, after some cancellation, the expression for anomalous resistivity in Equation (173) reduces to the following simple form in cgs units (with $\eta_{a}$ in seconds):

$$
\eta_{a}=4 \pi \sqrt{\frac{\pi}{2}} \frac{m_{e}}{m_{i}} \frac{1}{\omega_{L H}}\left(\frac{V_{d}}{V_{\text {th } i}}\right)^{2}\left(T_{e} \ll T_{i}\right)
$$

Here $V_{\text {th } i}$ is the ion thermal velocity, and $\omega_{L H}=\sqrt{\omega_{c i} \omega_{c e}}$ is the lower hybrid gyrofrequency. This expression for anomalous resistivity in the magnetoplasma gradient's current layer constitutes an upper bound, which is limited by the available drift kinetic energy in the electron's azimuthal drift current in the macroscopic rest frame of the ions. In view of our somewhat different derivation, it is satisfying that Equations (173) and consequently (176) for $\eta_{a}$ agree exactly with Equation (40) in the paper of Davidson and Gladd (Ref. 18). The upper bound on the fluctuation-electric-field energy density is called the Fowler bound as discussed in that paper.

It is sometimes convenient to carry out basic calculations in cgs units rather than mks units. When applied to resistivity, the cgs unit is seconds. Thus, the anomalous resistivity in Equations (176), (179), (196), and (198) all manifestly have identical dimensions of inverse frequency and hence are immediately recognized as being in terms of cgs units. The same is true as well of Equation (193) in the next subsection.
To convert any one of these to resistivities measured in the mks unit, ohm-meters, multiply these expressions by $9 \cdot 10^{9}$.

The electron collision frequency associated with $\eta_{a}$ in Equation (176) proves to be

$$
v_{a}=\sqrt{\frac{\pi}{2}} \frac{\omega_{p i}^{2}}{\omega_{L H}}\left(\frac{V_{d}}{V_{\mathrm{th} i}}\right)^{2}
$$

wherein $\omega_{i p}=\sqrt{4 \pi\langle n\rangle q^{2} / m_{i}}$ is the ion plasma frequency.

However, a critical refinement of the Fowler upper bound is required, as observed by Davidson (Ref. 23). In a regime often encountered in magnetoplasmas, which includes magnetic nozzle parameters of interest here, the averaged waveenergy density $W_{\text {wave }}$ far exceeds the energy density in the fluctuating electric field $W_{\mathrm{fl}}^{E}$. This regime of interest has $\omega_{p e}^{2} \gg \omega_{c e}^{2}$ (or $3 \cdot 10^{9} n \gg 3 \cdot 10^{14} B^{2}$ in cgs units, with $n$ in $\mathrm{cm}^{-3}$ and $B$ in gauss).

The energy density in the fluctuating electric field is only part of the total wave energy. It is related to the total waveenergy density as follows, according to Reference 23:

$$
W_{\mathrm{fl}}^{E}=\left(\frac{\omega_{c e}^{2}}{\omega_{p e}^{2}}\right) W_{\mathrm{wave}} \ll W_{\text {wave }}
$$

The reason the wave energy $W_{\text {wave }}$ far exceeds the energy in the fluctuating electric field $W_{\mathrm{fl}}^{E}$ is that the totality of wave energy includes contributions not only from $W_{\mathrm{fl}}^{E}$, but also from energy involved in the fluctuating particle motions themselves. The latter contribution actually proves much larger when $\omega_{p e}^{2} \gg \omega_{c e}^{2}$. In turn, the electron-drift kinetic energy constitutes the source for the total wave energy. In other words, the "Fowler bound" should be supplanted by the "wave-energy bound."

Following Davidson (Ref. 23), who states (without a clear physical justification) that only half the electron-drift energy goes into the wave energy, it is apparent from Equation (178) that a factor $\left[1 / 2 \omega_{c e}^{2} / \omega_{p e}^{2}\right]$ has to be inserted into Equation (176). Furthermore, Davidson chooses to express electron-drift velocity in terms of ion gyroradius in the limit $T_{e} \ll T_{i}$; namely, $V_{d} / V_{\text {th } i}=1 / 2 a_{i} / x_{n}$. Here, $1 / x_{n}=\left|n^{-1} \partial_{x} n\right|$ is the reciprocal radial gradient length. Equation (176) thereby becomes modified so as to yield a much smaller upper bound on the anomalous resistivity: 


$$
\eta_{a}=\left(\frac{\pi}{2}\right)^{3 / 2} \frac{\omega_{c e}^{2}}{\omega_{p e}^{2}} \frac{m_{e}}{m_{i}} \frac{1}{\omega_{L H}}\left(\frac{a_{i}}{x_{n}}\right)^{2} \quad\left(\mathrm{~T}_{\mathrm{e}} \ll<\mathrm{T}_{\mathrm{i}}\right)
$$

This is essentially the result stated by Davidson in the first section of his paper (Ref. 23). It can be shown to correspond to an electron collision frequency

$$
v_{a}=\frac{1}{8} \sqrt{\frac{\pi}{2}} \omega_{L H}\left(\frac{V_{d}}{V_{\mathrm{th} i}}\right)^{2}
$$

scaling as $\omega_{L H}$, whereas $v_{a}$ obtained from the Fowler bound (Eq. (177)) scales as $\left(1 / \omega_{L H}\right)$.

Later, the result Equation (179) will be compared with two published computer simulations of nonlinear evolution and concomitant radial diffusive transport of plasma particles. In this context, it should be noted that Davidson's derivation of Equation (179) had to rely upon the three approximations mentioned earlier: the local approximation, the electrostatic approximation, and the approximation of unmagnetized ions. Moreover, the derivation of Equation (179) in Reference 23 does not include some other features that are automatically incorporated in the computer simulations, such as wave-wave interactions.

\subsection{Alternative Models for Saturation of LHD Microturbulence}

Equation (173) indicates that the microturbulent resistivity $\eta_{a}$ depends fundamentally on the quantity of fluctuations squared in the electric field; specifically, $\eta_{a}$ depends upon the average energy density (second-order small) of electric field fluctuations, namely, $W_{\mathrm{fl}}^{E}=\left\{\left\langle\left\{\delta E_{y}\right\}^{2}\right\rangle / 8 \pi\right\}$. More complete models of quasi-linear theory include many modes, not just one. Alternative hypotheses have been proposed for saturation mechanisms for $W_{\mathrm{fl}}^{E}$, which include trapping of ions in the electrostatic wave-potential, electron resonance broadening, wave-wave coupling of growing waves to damped waves, and individual particle-orbit modification by the growing wave (see discussions in Refs. 14, 20, and 24 to 27). Each mechanism has had its advocates. Within the realm of plasma theory, the final arbiter of the various nonlinear-saturation hypotheses would be a well-done computer simulation with examples covering broad ranges of the relevant parameters.

In this section, we provide an overview of two iontrapping models, one using an energy method and another based on a time-scale method. Ion trapping, being intuitively obvious, was one of the first saturation mechanisms to be suggested. We also summarize the results of two other models, which proved to be in surprising agreement with the wave-energy-bound model. It is understood that all calculations are being done in the local longitudinal reference frame of the edge plasma. Also, ions are assumed to be singly charged in this subsection. We then conclude this subsection by summarizing the results of two nonlinear computer simulations of LHD microturbulence, one of which avoids two of the approximations mentioned above and the other of which avoids all three. Their results for the parameter dependencies of $\eta_{a}$ bear a striking resemblance to the anomalous-resistivity prediction of the quasi-linear model with the wave-energy bound. Unfortunately, there apparently is as yet no definitive agreement on the value of the numerical coefficient. Moreover, this numerical information is needed for optimization of magnetic nozzle design, so as to minimize attachment.

The idea behind the energy-based ion-trapping saturation mechanism is that if the traveling drift-wave potential accrues too much mass, the wave will be slowed and the synchronous dynamics that facilitates its growth will eventually be spoiled. The possible importance of the iontrapping mechanism can be estimated as follows: If the electric field fluctuation energy density, evaluated according to the wave-energy bound $W_{\mathrm{fl}}^{\text {wave }}$ is smaller than the electric field fluctuation energy from ion trapping $W_{\mathrm{fl}}^{\mathrm{it}}$ then the wave-energy-bound level of electric field fluctuations will be reached first and will saturate. The fluctuation level for ion trapping then would not be energetically accessible.

The kinetic energy of a thermal ion relative to a traveling drift wave of phase velocity $V_{\mathrm{ph}}$ is

$1 / 2 m_{i}\left(V_{\mathrm{th} i}-V_{\mathrm{ph}}\right)^{2}=1 / 2 m_{i} V_{\text {th } i}^{2}(1-\Phi)^{2}=T_{i}(1-\Phi)^{2}$

where $T_{i}$ is the ion temperature, and the ratio of wave phase velocity to ion thermal velocity is $\Phi=V_{\mathrm{ph}} / V_{\mathrm{th} i}$. If the electrostatic potential-energy pulse experienced by a representative co-moving ion $q\{\delta \varphi\}$ reaches the above level of kinetic energy, then the traveling wave potential can begin to drag ions along with it. The required electrostatic potential fluctuation $\{\delta \varphi\}$ is therefore

$$
\{\delta \varphi\} \approx \frac{T_{i}}{q}(1-\Phi)^{2}
$$

The electric field fluctuation is related to the fluctuation of electrostatic potential by $\{\delta E\} \approx k\{\delta \varphi\}$, where $k$ is essentially the azimuthal mode wave number of the fluctuation. Also, the field fluctuation energy density from ion trapping is on the order of $W_{\mathrm{fl}}^{\mathrm{it}} \approx\{\delta E\}^{2} / 8 \pi$. Therefore, the electric field energy-density level associated with ion trapping is

$$
W_{\mathrm{fl}}^{\mathrm{it}} \approx \frac{k^{2}}{8 \pi} \frac{T_{i}^{2}}{q^{2}}(1-\Phi)^{4}
$$


On the other hand, the electric field fluctuation energy density from the wave-energy bound is (suppressing Davidson's factor of $1 / 2$ )

$$
W_{\mathrm{fl}}^{\mathrm{wave}} \approx \frac{\omega_{c e}^{2}}{\omega_{p e}^{2}} \frac{1}{2} m_{e}\langle n\rangle V_{d}^{2}=\frac{\omega_{c i}^{2}}{\omega_{p i}^{2}} \frac{1}{2} m_{i}\langle n\rangle V_{d}^{2}
$$

The ratio of field fluctuation energy density from the waveenergy bound (Eq. (184)) to that from the ion trapping Equation (183) can be written as follows, after a bit of manipulation that utilizes the expressions for $\omega_{c i}$ and $\omega_{p i}$ :

$$
\frac{W_{\mathrm{fl}}^{\text {wave }}}{W_{\mathrm{fl}}^{\text {it }}} \approx \frac{4}{k^{2} a_{i}^{2}} \frac{V_{d}^{2}}{V_{\mathrm{thi}}^{2}} \frac{1}{(1-\Phi)^{4}}
$$

As the LHD wave of interest has its phase velocity $V_{\text {ph }}=$ $\operatorname{Real}(\omega) / k$ on the order of the drift velocity $V_{d}$ to within a constant $C_{\Pi}<1$ (for example, see Ref. 19, nonresonant case), Equation (185) will be written as

$$
\frac{W_{\mathrm{fl}}^{\text {wave }}}{W_{\mathrm{fl}}^{\text {it }}} \approx \frac{4}{k^{2} a_{i}^{2}} \frac{V_{d}^{2}}{V_{\mathrm{th} i}^{2}} \frac{1}{\left(1-C_{\mathrm{II}} V_{d} / V_{\mathrm{th} i}\right)^{4}} \text { where } \mathrm{C}_{\mathrm{II}}<1
$$

In the low-drift-velocity regime of interest (but still the nonresonant case), $V_{d}^{2} \ll V_{\text {th } i}^{2}$ in Equation (186). This strong inequality signifies a gradient length $x_{n}$ larger than an ion gyroradius $a_{i}$. Moreover, recall Equation (144b), which gives the wave number of the most rapidly growing LHD mode. It has the order of magnitude of $k \sim 1 / a_{e}$. Then, in Equation (186), the product $k^{2} a_{i}^{2}$ becomes on the order of $m_{i} / m_{e} \gg 1$. Hence, the numerator in Equation (186) is somewhat smaller than 1 , and the denominator is very large. It appears, therefore, that $W_{\mathrm{fl}}^{\text {wave }} \ll W_{\mathrm{fl}}^{\mathrm{it}}$, so that the iontrapping level of electric field fluctuations is not energetically possible in the regime of low drifts and broad gradients. Moreover, even in the opposite limit of a sharp gradient, $V_{d} \gg V_{\text {th }} i$, ion trapping according to Equation (186) is again not possible. It may be that energy-based ion trapping becomes marginally competitive in a narrow velocity interval centered at $V_{d} \approx V_{\text {th } i}$. It is clear that this energy-based model of ion trapping requires a single dominant wave.

There also is a different and simpler criterion for ion trapping, which will be aluded to below. It is based upon a time scale argument rather than an energy argument. The idea is that if a linearly small growing wave exists with growth rate $\gamma$ that is dominant over all other waves, then wave saturation occurs when the bounce frequency of an ion in the dominant wave potential $\omega_{\text {bnce }}$ exceeds the linear-phase growth rate; thus, $\omega_{\text {bnce }}>\gamma$ is the criterion. From Newton's equation of motion $(F=m a)$ of an ion in the dominant wave potential, one easily estimates $\omega_{\text {bnce }}^{2} \approx\left(q_{i} / m_{i}\right) k\{\delta E\}$, where $k$ is the wave number for the azimuthal direction and $\{\delta E\}$ is the fluctuating electric field in the azimuthal direction. The critical level for ion trapping then is $\{\delta E\} \approx \gamma^{2} m_{i} / q k$, where $\gamma$ is the dominant linear-phase growth rate as given, for example, in the collisionless case by Equation (144). A narrow wave spectrum is again required for this form of ion trapping. The anomalous resistivity discussed in the present report will not be related to this particular criterion, as the latter may require a critical damping condition made possible by frequent electron collisions with ions (see discussion below regarding Refs. 25 to 27). Nevertheless, the time-scale-based mechanism of ion trapping cannot be definitively ruled out.

Gary invoked electron resonance broadening as a secondorder mechanism of LHD-mode saturation. See the discussion in Reference 24, and references therein where it is believed that, nonlinearly, a large fraction of the electron distribution function is brought into near-resonance with the waves and extracts energy from them. In the same paper, he also applied that technique to several other kinds of microinstabilities that could be expected in magnetoplasma gradients confined by magnetic pressure, notably including the "universal instability," an oblique mode. His calculations are carried out analytically, and his result for electron resistivity from LHD microinstabilities proves to be practically the same as that obtained from the wave-energy bound in Reference 23.

Gary's calculations on the LHD instability in Reference 24 are restricted within the local approximation, the electrostatic approximation, and the unmagnetized ion approximation. Also, those calculations ignore wave-wave coupling, as does Reference 23. Gary's employment of electron resonance broadening to calculate saturation of turbulence and anomalous resistivity has been criticized by Drake et al. (Ref. 26) on the grounds that the model is restricted to being electrostatic.

Gary actually finds that anomalous resistivity from the oblique "universal mode" (in which the wave vector is not perpendicular to the magnetic field) exceeds that from the LHD instability. However, Gary's theory is electrostatic and therefore is restricted to zero $\beta$. Other work on the universal mode has shown that this mode is stable when $\beta$ exceeds a few percent (possibly 14 percent). Electromagnetic theory of the universal instability was addressed in References 28 to 30 . We infer that the universal mode should not be of principal importance in the edge layer of the propellant wherein $\beta$ is not small compared to 1 and moreover in which $\beta$ has a large range of values. Whether the universal mode would be of some secondary importance in a low-density plasma halo extending out into the magnetic nozzle field is an open question. 
Drake et al. (Ref. 26) followed the progression of "nearest neighbor" wave-wave couplings by means of numerical computation. Nearest neighbor modes are separated by the wave vector of the fastest growing linearized instability, known as the "pump" wave. The LHD waves were driven by resonant ions. Saturation of the resulting turbulence $(\mathbf{k} \cdot \mathbf{B}=$ 0 ) was ultimately obtained by electron damping at short wavelengths, either by $\nabla B$ drift-resonant electrons or by electron collisions (electron viscosity and electron-ion collisions).

Unlike the simulation in Reference 14 that is described below, that in Reference 26 is not a first-principles simulation. Rather, it is based upon reduced-model equations obtained from prior physical reasoning. Thus, there are two simplifying restrictions: to $T_{e}=0$ and to unmagnetized ions. There is also a simplification to nonlinear coupling of just nearest neighbor modes, which ultimately requires justification (in their appendix) by the presence of electron damping. Finally, the model features the spreading of energy in modespace, implying the generation of a broad spectrum of modes that is not suitable for the phenomenon of ion trapping. On the other hand, the model in Reference 26 is electromagnetic; hence, it is valid for nonzero $\beta$. Furthermore, the authors do simulate examples in which $V_{d}<V_{\text {th } i}$, unlike the examples presented in Reference 14, which are restricted to $V_{d} \approx V_{\text {th } i}$.

From their electromagnetic standpoint in Reference 26, Drake et al. criticize the electrostatic electron-resonance broadening theory of Gary (Ref. 24) by claiming that such a process in an electrostatic model can only shuffle energy back and forth between modes without producing true dissipation and concomitant saturation of turbulence. They claim that to produce saturation, an electromagnetic model is required with specific wave-particle resonances. They also point out that, within the electromagnetic model, magnetic energy effectively constitutes a very large reservoir of free energy, thereby invalidating the wave-energy-bound concept invoked in quasi-linear theory that the wave energy is limited by the available electron-drift kinetic energy. (Thus, it is implied in Ref. 26 that a resistively broadened plasma edge gradient can be steepened anew by the continual application of external magnetic pressure.)

In view of this remark, it is most interesting that the anomalous resistivity implied in Reference 26 has the same parameter dependencies obtained by Davidson (Ref. 23), albeit with a larger numerical factor. This result of Drake et al., which is not explicitly provided by them, will be derived now, based upon Equation (48) in Reference 26.

The crossfield particle diffusivity $D_{\perp}$ can be set within the context of the usual density-gradient-driven diffusion equation, namely

$$
n \mathbf{V}_{\perp}=-D_{\perp} \nabla n
$$

At the same time, one has radial force balance within the plasma gradient, neglecting inertia (hence, slow diffusion). This can be written as

$$
\nabla P_{\text {tot }}=c^{-1} \mathbf{J} \times \mathbf{B}
$$

wherein $P_{\text {tot }}$ is the total plasma pressure. (It is expeditious to use cgs units here, with $c$ the speed of light in vacuum.) Invoking the simple form of Ohm's law, the azimuthal current density is related to the azimuthal motional electric field by

$$
\mathbf{J}=\sigma\left(c^{-1} \mathbf{V}_{\perp} \times \mathbf{B}\right)
$$

These three macroscopic equations above may be easily combined to yield the relation between the particle diffusion coefficient $D_{\perp}$ and the resistivity $\eta=1 / \sigma$. For uniform temperatures of electrons and ions, that relation is

$$
D_{\perp}=D \frac{1}{2} \beta_{i}\left(1+\frac{T_{e}}{T_{i}}\right)
$$

where the resistive diffusivity (in cgs units) is

$$
D_{\eta}=\left(\frac{c^{2}}{4 \pi}\right) \eta
$$

Now, Drake et al. (Ref. 26) extract the following expression for the crossfield particle diffusivity from their simulations, notably including the value of the numerical factor $C_{\text {Drake: }}$

$D_{\perp}=C_{\text {Drake }} a_{\mathrm{es}}^{2} \omega_{L H}\left(\frac{V_{d}}{V_{\text {th } i}}\right)^{2}\left(\right.$ where $\left.C_{\text {Drake }}=2.4\right)$

Here, from their Equation (48) (in Ref. 26) as extracted from the simulations, $C_{\text {Drake }}=2.4$. The "strange" gyroradius $a_{\mathrm{es}}$ ("es" represents "electron-sound") is defined by $a_{\mathrm{es}}=a_{i} \sqrt{m_{e} / 2 m_{i}}$, where $a_{i}$ is the thermal ion gyroradius, $a_{i}=V_{\text {th } i} / \omega_{c i}$. Although the Drake et al. model is electromagnetic, they claim no explicit $\beta$ dependence for this $D_{\perp}$.

One can directly solve Equation (190) for the resistivity, using Equations (191) and (192) and the definition of $a_{\mathrm{es}}$. In turn, that result can be manipulated further, using the following identities: 


$$
\begin{gathered}
\beta_{i}=\frac{V_{\mathrm{th} i}^{2}}{V_{A}^{2}} \\
\frac{c^{2}}{\omega_{p i}^{2}}=\frac{V_{A}^{2}}{\omega_{c i}^{2}} \\
\frac{\omega_{c i}^{2}}{\omega_{p i}^{2}} \frac{m_{i}}{m_{e}}=\frac{\omega_{c e}^{2}}{\omega_{p e}^{2}} \\
\omega_{L H}^{2}=\omega_{c e} \omega_{c i}
\end{gathered}
$$

Also, in cgs units, we have to use the following definitions (with $P_{\text {tot }}=n T_{e}+n T_{i}$ ):

$$
\begin{gathered}
\beta_{i}=\frac{8 \pi P_{i}}{B^{2}} \\
V_{\mathrm{th} i}^{2}=\frac{2 T_{i}}{m_{i}} \\
V_{A}^{2}=\frac{B^{2}}{4 \pi n m_{i}} \\
\omega_{p}^{2}=\frac{4 \pi n q^{2}}{m_{s}} \\
\omega_{c}=\frac{q B}{m_{s} c}
\end{gathered}
$$

Here, $T$ is the temperature in ergs.

The final result for the resistivity, now noted as the anomalous resistivity, $\eta=\eta_{a}$, can then be written exactly as follows:

$$
\eta_{a}=\frac{4 \pi C_{\text {Drake }}}{\left(1+T_{e} / T_{i}\right)} \frac{\omega_{c e}^{2}}{\omega_{p e}^{2}} \frac{m_{e}}{m_{i}} \frac{1}{\omega_{L H}}\left(\frac{V_{d}}{V_{\mathrm{th} i}}\right)^{2}
$$

Drake et al. (Ref. 26) sets $T_{e}=0$ in their model, as does Davidson in Reference 23. Except for the numerical factor, this expression is then identical to the zero- $\beta$ expression of Davidson, Equation (179). After converting Equation (179) in terms of the current drift velocity $V_{d}$, the numerical factor in Equation (179) is about 7. In Equation (195) above, for $C_{\text {Drake }}=2.4$, the numerical factor $4 \pi C_{\text {Drake }}$ is $\sim 30$.
The anomalous resistivity inferred in the Brackbill et al. paper (Ref. 14) discussed below again proves to be given by the identical expression to the above except for the numerical coefficient, provided that the ion- $\beta$ factor is neglected. In the Brackbill et al. paper, the largest value of the numerical coefficient in $\eta_{a}$, as extracted from the simulations, is stated to be 0.4. Thus, the numerical coefficient in Drake et al. is almost 2 orders of magnitude larger than that in Brackbill et al. This is a significant discrepancy in view of the fact that the results of Drake et al. compare somewhat favorably with measurements on a theta pinch implosion, as will be discussed next.

The output of Drake et al. is compared with measurements on theta pinch implosions performed by Fahrbach et al. (Ref. 27). In so doing, Drake et al. remark that it is important to include electron-ion collisions in the theory, which they do. At the same time, they emphatically maintain that their Equation (48) (in Ref. 26) should still be interpreted as the collisionless expression for $D_{\perp}$ because their simulations show that crossfield particle transport is very insensitive to the magnitude of electron dissipation.

The actual comparisons made with the experiments of Fahrbach et al. are not the particle diffusion coefficient $D_{\perp}$ or the resistively broadened theta pinch profiles; rather, they are the shape and position of the mode spectrum and the amplitude of the plasma density fluctuations. The spectral comparisons agree very well; for example, the predicted down-shift in the peak of the wave number spectrum differ by only about a factor of 2 . The fractional amplitude of the density fluctuations differ more, but still within an order of magnitude: 0.034 versus 0.014 at the lowest filling pressure and 0.014 versus 0.0023 at the intermediate filling pressure (where the greater values represent the theoreticals). (Note that anomalous resistivity depends on the squares of those fluctuation values.) At the highest filling pressure in the experiments, the theoretical model finds that the system is very close to marginal stability for LHD modes because of electron collisions with ions; hence it should be dominated by a single mode, and ion trapping should probably be responsible for the saturated amplitude. In fact, the experimentalists invoke the time-scale-based ion trapping mechanism to explain their results at all three filling pressures.

In view of the several competing mechanisms proposed for nonlinear saturation, recourse to first-principles simulations of the evolution of magnetoplasma gradients is useful. Such simulations are unbiased as to mechanisms. Brackbill et al. (Ref. 14) developed full-particle (both electron and ion) electromagnetic simulations (hence, capable of addressing nonzero $\beta$ ). The considered equilibria had $T_{e} / T_{i}<1$, corresponding to our Equation (144) and Davidson's case (Ref. 23), but the electron and ion temperatures were allowed to evolve self-consistently with the microturbulence. As in Reference 26, a major restrictive assumption was that 
only modes with wave vectors perpendicular to the magnetic field were allowed. Unlike Reference 26 , however, only examples with $V_{d} \approx V_{\text {th }} i$ were simulated, although this restriction was not required in principle.

In Reference 14, the microturbulent behavior was simulated in a self-consistent manner over the entire width of the plasma density gradient, so there was no local approximation. Particle orbits were self-consistently calculated in the ambient zero-order magnetic and electric fields as well as in the nonlinearly fluctuating fields produced by the particle motions themselves. Fluid equations were utilized in an intermediate time step to advance the fields. There was no "unmagnetized ion" approximation. The magnetic field profile ranged from a large external value to zero deep in the plasma, at the center of the current layer.

To our knowledge, this is the only published firstprinciples electromagnetic full-particle simulation of LHDrelated microturbulence in a global-configuration magnetoplasma gradient, from which a practical formula for the internal anomalous resistivity $\eta_{a}$ has been extracted. A paper was recently published that included oblique modes, but the principal interest there was three-dimensional magnetic reconnection across the current layer; see Reference 31 . The configuration addressed in the Brackbill et al. paper (Ref. 14) is relevant to the magnetic nozzle device because the internal plasma is confined by external magnetic pressure. If no such practical formula for $\eta_{a}$ were forthcoming, then simulations of microturbulence in the magnetic nozzle would have to be carried out in an iterative manner at many locations along the propellant's flow field within a resistive MHD simulation, which seems impractical within normally available resources.

A note of caution, however, is that the paper of Brackbill et al., as in related work dealing with theta-pinch implosions, differs in a fundamental way from the magnetic nozzle. As emphasized by Turchi, in virtue of the longitudinal flow of propellant the magnetic nozzle has "new" plasma coming from upstream, which is continually entering previously disturbed regions of the plasma gradient. Thus, broadening of the plasma-field interface region in the magnetic nozzle may differ from the results of Reference 14.

Since $V_{d} / V_{\text {th }} \approx 1$ in the initial equilibria in Reference 14 , it is somewhat surprising that the same parameter dependencies (except for the $\beta$ dependence) are inferred for the anomalous resistivity as are found in References 23 (Davidson) and 26 (Drake). However, both Davidson's and Drake's numerical coefficients in $\eta_{a}$ are substantially larger than observed in the Brackbill et al. simulations.

Data from the simulations in Reference 14 is somewhat sparse and exhibits significant scatter. Nevertheless, the overall trends of the data points from Reference 14 (which also include the results of earlier simulations) are consistent with the $\eta_{a}$ expressions in the papers of Davidson (Ref. 23), Gary (Ref. 24), and Drake et al. (Ref. 26). In fact, the practical formula for anomalous resistivity in the Brackbill paper was inferred (with an adjustable constant) by comparing the parameter dependencies of the particle heating rates in the simulation with the second-order heating rates obtained analytically by Gary. (Note, however, that Gary employed an electrostatic zero- $\beta$ model.)

We shall write down the simulation-based formula for $\eta_{\mathrm{a}}$ as set forth in the Brackbill paper (Ref. 14), except that a factor $1 / \beta_{i}$ shall be suppressed. We believe that this inverse$\beta$ factor is not well founded. The ion $\beta$ is defined by $\beta_{i}=$ $8 \pi P_{i} / B^{2}$. The reasons for our reluctance to accept this $\beta$ factor, besides its absence in equation (48) of Drake et al. (Ref. 26), is that the Brackbill simulation results are compared with a template based upon a zero-beta electrostatic model, so that an inference about the beta dependence is not logically possible. In any event, the ion $\beta$ would be of order 1 within the magnetically confined plasma gradient.

The formula suggested in Reference 14 for the effective anomalous resistivity then can be written without $\beta_{i}$ as

$$
\begin{aligned}
\eta_{a}=C_{\mathrm{Brack}} \frac{\omega_{c e}^{2}}{\omega_{p e}^{2}} \frac{m_{e}}{m_{i}} \frac{1}{\omega_{L H}}\left(\frac{V_{d e}}{V_{\text {th } i}}\right)^{2} \\
\text { where } 0.1<C_{\text {Brack }}<0.4
\end{aligned}
$$

This symbol $C_{\mathrm{Brack}}$ corresponds to $4 \pi C_{\text {Drake }}$ in the Drake et al. discussion above. The largest value of $C_{\mathrm{Brack}}$ obtained in the Brackbill et al. simulations was $C_{\mathrm{Brack}}=0.4$, and the smallest was $C_{\mathrm{Brack}}=0.1$. The range of $C_{\mathrm{Brack}}$ was related to the stage of evolution of the microturbulence and concomitantly of the evolving plasma gradient. When comparing this $\eta_{a}$ to classical resistivity $\eta_{\mathrm{cl}}$, we shall refer to the largest value of $C_{\mathrm{Brack}}, 0.4$. It is an open question as to what stage of evolution, in terms of Reference 14, is appropriate in the magnetic nozzle, as a given plasma element travels along the magnetic nozzle field in a macroscopic state of stationary flow.

Reverting from the velocity ratio to the ratio of ion gyroradius to gradient scale length, and in the limit of small $T_{e} / T_{i}$, the simulation result, Equation (196), is compared with Davidson's quasi-linear result, Equation (179). Since $\left(V_{d} / V_{\mathrm{th}}\right)^{2}=1 / 4\left(a_{i} / x_{n}\right)^{2}$ when $T_{e}=0$, the constant $C_{\mathrm{Brack}}$ in the first-principles simulation result Equation (196) is about an order of magnitude smaller than that produced by the quasilinear wave-energy-bound model represented in Equation (179). The simulations in Reference 14 had $T_{i}$ somewhat larger than $T_{e}$ (see Table I in Ref. 14), as in Davidson's analytical case (Ref. 23), so the temperature ratio probably cannot account for this discrepancy. The discrepancy might have been understandable from the expectation that spreading of available energy within wave-vector space by wave coupling and energy loss to damped modes in the simulation would lead to a reduced level of fluctuations. However, 
Drake et al. include wave coupling, yet find a much larger numerical coefficient for anomalous resistivity $\eta_{a}$ than does Brackbill et al.

Drake et al. (Ref. 26) pointed out that if there were a channel for magnetic energy to be continually converted to plasma energy, then the wave-energy bound used in quasilinear theory, as limited by the electron-drift kinetic energy in the azimuthal current layer, would not be applicable. In principle, the level of microturbulent fluctuations could then increase beyond that value from the wave-energy bound, due to the essentially infinite supply of magnetic energy. In fact, the simulations in Reference 14 did find that magnetic energy was being continually converted into plasma energy. However, it was apparently being converted directly into thermal energy of the particles, rather than going into an increased level of microturbulent fluctuations. This result is understandable from the point of view that continually applied external magnetic pressure just keeps squeezing and heating the plasma.

\subsection{Comparison of Anomalous and Classical Resistivities}

The simulation result Equation (196) will now be compared with classical resistivity. However, we should be mindful that this may constitute an underestimate of anomalous resistivity in view of the results of Drake et al., which have the same parameter dependence but with a significantly larger numerical coefficient. Moreover, the latter result compares favorably with some experimental measurements. It is understood that all calculations are being done in the local longitudinal reference frame of the edge plasma.

For comparison to classical resistivity, it proves convenient to rewrite the anomalous resistivity simulation result, Equation (196). The following identities are utilized:

$$
\begin{gathered}
\left(\frac{\omega_{c e}^{2}}{\omega_{p e}^{2}}\right)\left(\frac{m_{e}}{m_{i}}\right)=\left(\frac{\omega_{c i}^{2}}{\omega_{p i}^{2}}\right) \\
\omega_{L H}=\omega_{c i}\left(\frac{m_{i}}{m_{e}}\right)^{1 / 2} \\
\frac{c}{\omega_{p i}}=\frac{V_{A}}{\omega_{c i}} \\
\omega_{p i}\left(\frac{m_{i}}{m_{e}}\right)^{1 / 2}=\omega_{p e}
\end{gathered}
$$

Here, $V_{A}$ is the Alfven velocity. Invoking these identities, after some manipulation, Equation (196) can be written as

$$
\eta_{a}=C_{\mathrm{Brack}} \frac{V_{A}}{c} \frac{1}{\omega_{p e}}\left(\frac{V_{d}}{V_{\mathrm{th} i}}\right)^{2}
$$

Here, $c$ is the speed of light in vacuum, in centimeters per second. Numerical calculations of this anomalous resistivity shall be performed at a representative position within the plasma gradient.

To evaluate the factor $\left(V_{A} / c\right)$ in Equation (198), we utilize the cgs expression, $V_{A}=B / \sqrt{4 \pi n_{i} m_{i}}$. Mass $m_{i}$ is assumed to be that of the hydrogen ion. We wish to evaluate $\eta_{a}$ in Equation (198) at a representative position within the density gradient that constitutes the plasma-field interface layer. Midway within the gradient, magnetic pressure $\left(B^{2} / 8 \pi\right)$ is set to half the external magnetic ( $B_{\text {ext }}$ ) pressure; hence, $B^{2}=0.5$ $B_{\text {ext }}^{2}$. Also, ion number density $n_{i}$ is set to half the internal (core) number density $n_{\text {core }}$; hence, $n_{i}=0.5 n_{\text {core }}$. Then pressure equilibrium $B_{\text {ext }}^{2}=16 \pi n_{\text {core }} T$ is invoked across the entire layer width for external magnetic confinement of the internal plasma pressure. A uniform temperature $T$ (ergs) is assumed, with $T=T_{e}=T_{i}$. The result is $V_{A} / c=0.67 \cdot 10^{-4}$ $\sqrt{T(\mathrm{eV})}$, with the temperature $T(\mathrm{eV})$ now measured in electron volts. Similarly, the electron plasma frequency in Equation (198) is given in cgs units by $\omega_{p e}=\sqrt{4 \pi n_{p} q^{2} / m_{e}}=5.6 \cdot 10^{4} \sqrt{n_{p}}$, and the plasma number density $n_{p}$ in the gradient is again set to half the core density. Thus $n_{p}=0.5 n_{\text {core }}$. Then, from Equation (198), the anomalous resistivity $\eta_{a}$ at a representative position within the magnetically confined plasma gradient reads as follows:

$$
\eta_{a}=\left[1.7 C_{\text {Brack }}\right]\left(10^{-9}\right) \sqrt{\frac{T(\mathrm{eV})}{n_{\text {core }}}}\left(\frac{V_{d}}{V_{\text {th } i}}\right)^{2}
$$

The ratio of current drift velocity $V_{d}$ to ion thermal velocity $V_{\text {th } i}$ actually is profile dependent. The treatment of this quanity is discussed below. Of course, use of Equations (196) or (198) is preferred for $\eta_{a}$ in a resistive MHD simulation, so as to provide better resolution within the edge-plasma gradient.

The well-known classical resistivity $\eta_{\mathrm{cl}}$ across the magnetic field (Ref. 9) due to coulomb collisions of electrons with ions is practically independent of plasma number density. If we set $\ln \Lambda \approx 10$, which is typical, then $\eta_{\mathrm{cl}}$ will read as follows:

$$
\eta_{\mathrm{cl}}=1.1 \cdot 10^{-13} T(\mathrm{eV})^{-3 / 2}
$$


Both resistivities $\eta_{a}$ and $\eta_{\mathrm{cl}}$ are measured in seconds in cgs units. Multiplication by the number $9 \cdot 10^{9}$ converts them into mks values measured in ohm-meters.

Either classical or anomalous resistivity can be employed in a simple Ohm's law, to relate the azimuthal current density in the edge-plasma gradient layer to the motional azimuthal electric field $\mathbf{V} \times \mathbf{B}$ (the cross product of quasiradial diffusion velocity with longitudinal magnetic field). Their use in the simple Ohm's law, however, requires that the radial Hall voltage does not become shorted out by a flow of Hall current. As a practical matter, a resistive MHD simulation such as the MACH2 code can be set to run with the sum of the two resistivities. Then the dominant one will automatically be the effective resistivity.

For a more systematic impression of the integrity of the plasma-field interface, it is of interest to describe conditions under which microturbulent (anomalous) resistivity is the larger of the two. From Equations (200) and (199), the ratio of classical to anomalous resistivity, where temperature $T$ is in electron volts, is

$$
\frac{\eta_{\mathrm{cl}}}{\eta_{a}}=\frac{1}{1.5 C_{\mathrm{Brack}}}\left(10^{-4}\right) \frac{n_{\mathrm{core}}^{1 / 2}}{T^{2}} \frac{1}{\left(V_{d} / V_{\mathrm{th} i}\right)}
$$

Classical resistivity clearly dominates at lower temperatures and higher densities. The two are equal, however (again, when temperature $T$ is in electron volts), when

$$
T=\frac{1}{1.22 \sqrt{C_{\text {Brack }}}}\left(10^{-2}\right) \frac{n_{\text {core }}^{1 / 4}}{V_{d} / V_{\text {th } i}}
$$

Numerical examples for which $\eta_{\mathrm{cl}}=\eta_{a}$ are tabulated below for $C_{\mathrm{Brack}}=0.4$ and $T=T_{e}=T_{i}$. Our earlier analysis of the ion gyroradius relative to the initial interface width also confirmed the approximate constancy of that ratio (i.e., $\left.a_{i} / x_{n}\right)$ from breech to throat. Note $x_{n}$ is the characteristic density gradient scale length, which is defined in terms of the ratio of the local ion number density to the local gradient of the ion number density. A special derivation is needed to relate that ratio, $a_{i} / x_{n}$ to the velocity ratio $V_{d} / V_{\text {th } i}$ by virtue of the MHD equilibrium relation in the quasi-radial direction across the flux surfaces, within the interface layer. In this derivation it is assumed that the electron and ion temperatures are equal and uniform. Accordingly, we have chosen the example $V_{d} / V_{\text {th } i}=a_{i} / x_{n}=1 / 3$.

There is a lack of precision, however, because the current drift velocity $V_{d}$ (or also ion gyroradius $a_{i}$ ) depends upon location within the plasma-field mixing layer, and also because its thickness $\delta\left(=x_{n}\right)$ as a multiple of $\left(c / \omega_{p}\right)$ is not precisely known (see Appendix I). Hence, a slightly greater value of $V_{d} / V_{\text {th } i}=a_{i} / x_{n}=1 /(2.5)$ is also considered.

Figure 4 presents temperatures $(\mathrm{eV})$ below which $\eta_{\mathrm{cl}}$ (with $\ln \Lambda=10$ ) is larger than $\eta_{a}$ (Ref. 14 with $C_{\mathrm{Brack}}=0.4$ ), for a given ion number density $\left(\mathrm{cm}^{-3}\right)$. The two values for the ratio $V_{d} / V_{\text {th }} i$ are plotted. The critical temperature according to Equation (202) is rounded to the nearest $10 \mathrm{eV}$.

The results in Figure 4 ought to be insensitive to position along the nozzle between breech and throat. That is because density $n$ at the throat is still about $2 / 3$ of its breech value (and $n^{1 / 4}$ is used), and temperature $T$ at the throat is still $3 / 4$ of its breech value. Thus, at a hydrogen-ion number density $10^{15} \mathrm{~cm}^{-3}$, an envisioned stagnation temperature of $50 \mathrm{eV}$ in the breech would represent a condition of classical resistivity, whereas 180 to $220 \mathrm{eV}$ would represent a marginal condition where the two resistivities are about equal. The $220 \mathrm{eV}$ range is, in fact, envisioned in a recent space-mission systems study (see Ref. 4).

In view of the results of Drake et al. (Ref. 26), the reader should be warned of the possibility that Figure 4 may

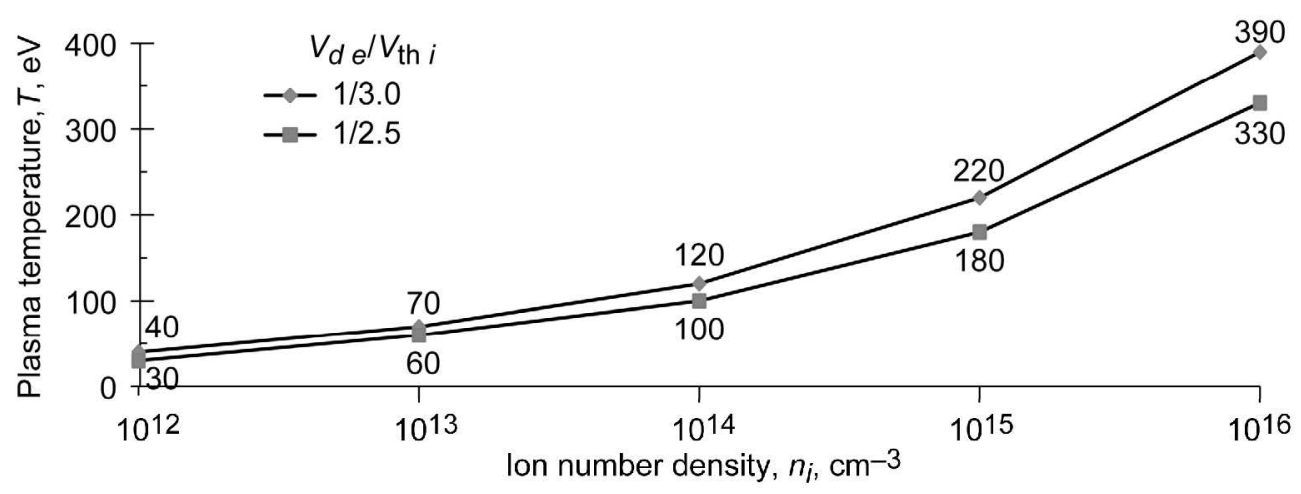

Figure 4.-Temperatures for which classical resistivity exceeds anomalous resistivity (for specific ratios of electron drift velocity $V_{d e}$ to ion thermal velocity $V_{\text {th }} i$ ). 
wrongly downgrade the importance of anomalous resistivity. The critical temperatures in this figure actually depend on $1 / \sqrt{C_{\mathrm{Brack}}}$. If $C_{\mathrm{Brack}}$ should have been 2 orders of magnitude larger, then the critical temperatures would be smaller by a factor 10 . If such were the case, then the anomalous resistivity $\eta_{a}$ would be totally dominant over the classical resistivity $\eta_{\mathrm{cl}}$ for most cases of interest. However, the fluctuation levels predicted by Drake et al. were somewhat too large compared with the experimental measurements, and it is the squares of those fluctuation levels that determine the anomalous resistivity.

\subsection{Rayleigh-Taylor- (RT-) Type Instabilities}

In this section, we primarily examine MHD surface instabilities of the flute-mode type, associated with adverse longitudinal curvature of the plasma boundary. To begin with, however, brief consideration is also accorded the unique conditions in the injection region associated with the initial plasma impact with the confining magnetic field.

Two distinct regions involve instabilities of the RT type. The usual one involves propellant flow along magnetic-field lines of gradual adverse curvature. An effective gravitational force field arises from centrifugal deceleration. In the same region there are separate pressure-driven modes with similar characteristics, but for which there is no effective gravity because there is no macroscopic centrifugal force field. The pressure-driven modes require a separate treatment, which is supplied below. In magnetic nozzle flow, a combined instability occurs driven both by centrifugal effects and pressure. Therefore, growth rates for modes of the combined type will also be derived. The treatment is based on surveying the forces acting on a plasma element as it is displaced outwards in a region of adverse magnetic curvature. Treatments in the literature appear to be more mathematical and less accessible.

For these instabilities, the vulnerable regions are where propellant approaches the throat of the nozzle and also near the nozzle's exit, where the diverging flow is redirected axially by straightening out the diverging magnetic field lines (Fig. 2). The most dangerous RT-type instabilities in these two regions are usually considered to be flute modes, with azimuthal wave vectors, because these modes do not bend magnetic field lines; hence, they are energetically easier to destabilize than oblique modes directed partially along $\mathbf{B}$.

The other region to consider is the nozzle entrance region where plasma first impacts the magnetic field. That process involves quasi-radial expansion of injected hot plasma (in the direction $\hat{\mathbf{x}}=\hat{\boldsymbol{\theta}} \times(\mathbf{B} / B)$ ) across the nozzle magnetic field. The plasma's subsequent sudden deceleration and redirection along $\mathbf{B}$ necessarily creates a propellant-flow streamline with sharp adverse curvature. The problem is first addressed in the nozzle entrance, to identify the degree of adverse curvature inherent in the injected edge-plasma streamlines. After that, the RT-type instabilities occurring further downstream are addressed.

\subsection{RT-Type Instabilities at Initial Impact With Magnetic Field}

Plasma impact at approximately normal incidence to a surrounding magnetic field has been studied experimentally, with theoretical validation, by Ripin et al. (Ref. 17). Laserproduced plasma was generated at an aluminum surface, and the plasma puff then expanded across an ambient magnetic field. Fine-scale radial protrusions of plasma were observed at the plasma radius where deceleration of the plasma front by the magnetic field began to occur, and they were attributed to RT unstable conditions of deceleration at the plasma-field interface. Extensions of the MHD model to include Hall effects (Huba, Ref. 32) improved the relation of observations to theory. Evolution of RT structures was followed into the nonlinear regime, both experimentally and theoretically. Finite Larmor radius (FLR) effects (Huba, Ref. 33) were not invoked in Reference 17, possibly because the plasma temperature was too low for them to be relevant. Also, early experiments on theta-pinch implosions were observed in the implosion-onset phase to contain fine-scale radial protrusions around the circumference (McKenna, Ref. 34), but no detailed identification was attempted.

In the magnetic nozzle, one might hope to mitigate RT instabilities by inserting propellant at grazing incidence to magnetic field lines, thus minimizing adverse curvature of the edge streamlines in the injection region. This point of view is adopted below. It will be seen, however, that the transition from oblique incidence of flow (relative to B) over to flow approximately along $B$ is so sudden that the usual MHD model is not adequate. A more detailed model is needed to describe (and enable a more rigorous investigation of) propellant flow in the plasma-field interface layer at the breech region of the nozzle, which would enable RT instabilities.

From the point of view of the single-fluid MHD model with $T_{e}=T_{i}$, the equation governing crossfield motion of plasma is, in mks units,

$$
\rho \frac{\mathrm{d} V_{x}}{\mathrm{~d} t}+\frac{\partial P}{\partial x}=J_{y} B_{z}=-\sigma V_{x} B_{z}^{2}
$$


This can be rewritten schematically as

$$
\frac{\mathrm{d} V_{x}}{\mathrm{~d} t}+\frac{V_{A}^{2}}{D} V_{x}=\frac{V_{\mathrm{th} i}^{2}}{x_{n}}
$$

The negative pressure gradient on the left in Equation (203a) has reappeared on the right in Equation (203b) as a positive term, and the negative resistive-drag term on the right has reappeared as a positive term on the left. The quasi-radial fluid velocity is $V_{x}, D$ is resistive diffusivity, $V_{A}$ is Alfven velocity in the gradient layer, and the reciprocal gradient length is $x_{n}^{-1}=\left|n^{-1} \partial_{x} n\right|=\delta^{-1}$. When the inertial term, $\mathrm{d} V_{x} / \mathrm{d} t$, is neglected, one recovers the quasi-radial resistive diffusion velocity that was identified near the beginning of Section 2.0. The magnetic flux is assumed to have no give because it has already been squeezed against the metallic wall (coil) by the plasma, and a state of stationary flow has already been reached. Equation (203b) is to be interpreted in a Lagrangian sense of following a given plasma element.

The solution of Equation (203b) is

$$
V_{x}=V_{x \text { core }} e^{-t / t_{\text {trans }}}+V_{x \infty}\left(1-e^{-t / t_{\text {trans }}}\right)
$$

The subscript "core" now refers to the initial value of the crossfield velocity when the considered element of plasma first encounters the magnetic field at the nominal plasmafield interface. The subscript " $\infty$ " signifies the eventual crossfield resistive-diffusion velocity attained at large times after the inertial transient has dissipated. $V_{x}$ is realistically assumed to be very small compared with $V_{x}$ core. The time interval over which the inertial transient lasts, $t_{\text {trans }}$, is the time during which there is appreciable crossfield velocity, in terms of the incident velocity of the plasma element.

$$
t_{\text {trans }}=\frac{D}{V_{A}^{2}}
$$

For parameters of interest, this transient lasts only a fraction of a nanosecond, which signifies that the macroscopic single-fluid MHD model is intruding into the purview of microphysical processes. This cautionary conclusion is further verified when one considers the radius of curvature of the trajectory of the considered edge-plasma element, which ordinarily would provide information relevant to growth rates of RT instabilities. Pursuant to calculating the longitudinal radius of curvature $R$, the distance the considered plasma element travels along B during time $t_{\text {trans }}$ is approximately

$$
z_{\text {trans }} \approx V_{\text {core }} t_{\text {trans }}=\frac{V_{\text {core }} D}{V_{A}^{2}}
$$

valid for a shallow (i.e., grazing) angle of incidence.

Let $\phi_{\mathbf{B}} \mathbf{v}(\mathrm{rad})$ denote the incident angle between $\mathbf{B}$ and $\mathbf{V}$ at the nominal plasma-field interface. Then, $\sin \phi_{\mathbf{B} \mathbf{V}} \approx \phi_{\mathbf{B} \mathbf{V}}$ $\approx V_{x \text { core }} / V_{\text {core }} \ll 1$. Simple geometrical considerations show that the radius of curvature $R$ of the trajectory can be expressed approximately as

$$
R \approx \frac{z_{\text {trans }}}{\phi_{\mathbf{B V}}}=\frac{V_{\text {core }} / V_{A}}{\phi_{\mathbf{B V}}} \frac{D}{V_{A}}
$$

Equation (207) shows that $R$ is very small for parameters of interest in this report, although the angle $\phi_{\mathbf{B V}}$ is moderately small (for grazing incidence). For example, $R \sim 0.01-0.001 \mathrm{~cm}$. This follows from typical values of the resistive diffusivity $D\left(\sim 10^{4} \mathrm{~cm}^{2} / \mathrm{s}\right)$, and of the Alfven velocity $V_{A}\left(\sim 10^{7} \mathrm{~cm} / \mathrm{s}\right)$, jointly producing a very small length $D / V_{A}$. (The impacting velocity $V_{\text {core }}$ is only smaller than $V_{\text {th }} i$ by a moderate area ratio, and $V_{\text {th } i} \sim V_{A}$ upstream of the throat. Hence, $V_{\text {core }} / V_{A}$ is only moderately small.) In reality, "microscopic" lengths properly incorporated into a more complete plasma model, such as the ion gyroradius $a_{i}$ or the ion skin depth $c / \omega_{p} i$, both $\sim 1 \mathrm{~cm}$, would provide lower bounds to $R$.

The single-fluid MHD model has proven inappropriate for a quantitative description of the initial trajectory of an edgeplasma fluid element at "magnetic impact," although it is incident at a shallow angle with magnetic flux at the interface. Nevertheless, the message is clear. From a macroscopic point of view, the plasma element's incident trajectory onto the external magnetic flux undergoes an instantaneous transition into motion practically along $B$, due to eddy current braking from electron resistivity.

A large RT growth rate, $\gamma_{R T}$, is implied by the small radius of curvature of the incident trajectory, at its point of impact with magnetic field. But the importance of RT instabilities in that region yet depends upon the time $t_{\text {cur }}$ during which the plasma element resides in the highly localized region of adverse curvature. The product $\gamma_{\mathrm{RT}} t_{\mathrm{cur}}$ determines the number of e-folds available for the RT instability in that region. The RT instabilities in the plasma-field impact region therefore should be examined with the use of appropriately detailed plasma models beyond the ordinary MHD fluidbased model (see, for example, Ref. 35). The incident internal streamlines that impact the external magnetic flux at the plasma-field interface in the injection region (breech) unavoidably acquire a segment of severe adverse curvature not treatable under ordinary MHD. 


\subsection{RT-Type Instabilities Downstream From Injection Region}

We turn now to the topic of RT instabilities downstream from the injection region but upstream of the throat, where the cross section of the plasma shrinks to form the throat. There, the transition to field-aligned flow has already been made, and the plasma propellant streamlines near the plasma-field interface closely adhere to the magnetic-field line shape because of the high electrical conductivity of the plasma. Section 3.0 addressed gradient-driven microinstabilities localized within the plasma-field interface, which cause broadening of the interface layer by resistive diffusion. Here, Section 4.0 addresses macroscopic instabilities that cause the interface to deform (to wrinkle) as an entity. This process of macroscopic interface deformation is a consequence of instability wavelengths that are large compared with the width of the interface layer. The practical consequence is a loss of nozzle-based control of the flow of propellant.

The usual fluid-based MHD model can be applied to describe RT flute instabilities, provided thermal excursions of individual particles are short compared to characteristic macroscopic lengths in the nozzle. Examples of such macroscopic lengths are the plasma radius $r$, the radius of curvature of field lines and/or streamlines $R$, and long flutemode wavelengths in the azimuthal direction $2 \pi / k$, with $k$ as the azimuthal wave number. It is similarly required that times for those thermal excursions be short compared to macroscopic times such as longitudinal transit times and instability growth times (e.g., see Ref. 9).

An example of a thermal excursion length across the magnetic field is the nominally small ion gyroradius, which takes place during a nominally short ion gyroperiod. An example along the field is the mean free path for ion-ion coulomb collisions, which transpires during a mean free time between ion-ion collisions. The electron mean free path is about the same as the ion mean free path. For cases of interest here, mean free paths $\lambda$ along $B$ are several centimeters (for $n \sim 10^{15} \mathrm{~cm}^{-3}$ and $T \sim 50$ to $100 \mathrm{eV}$ ) and are generally much smaller than meters.

Within the core plasma, from which the nozzle magnetic field has been excluded by the azimuthal current layer, the thermal excursions are governed by just the mean free paths and mean free times for coulomb collisions of charged particles. Thermal excursions in the core plasma are unrestricted by the magnetic field, and so they take place in all directions. If $\lambda$ is several centimeters, then not only the characteristic axial dimension should be at least tens of centimeters, but the radial dimension should be as well.

These restrictions on thermal excursions of individual particles are meant to ensure that any given fluid element retains its identity (undergoes very little random thermal dispersal) over the relevant macroscopic lengths and times of interest in the device. Then the use of a fluid model makes sense.
Sometimes, though, fluid-MHD simulations are applied to hot plasma configurations without raising cautions that the above restrictions on thermal excursions may not always be well satisfied. At lower densities and higher temperatures relative to the nominal working values of interest for this report, a kinetic multispecies model may have to supplant the basic single-fluid model of MHD. Such models have been developed in the magnetic confinement fusion (MCF) community, but for different purposes; nevertheless there is some expertise in the MCF community in global-kinetic modeling.

In the discussion here of RT instabilities downstream from the injection region, we shall assume that the conditions for a single-fluid model are fulfilled. An exception is made in Section 4.4, "Short-Wavelength Gravity-Driven Instabilities," where short-wavelength instabilities that are related to RT physics are discussed.

The present subsection first derives in a heuristic manner the RT-type instabilities in a static field-free plasma (without flow), confined by pressure equilibrium with the external magnetic field and situated within a region of adverse magnetic curvature. The derivation is carried out by considering the forces acting on a plasma element under the region of adverse curvature. Then the modification of the derivation to incorporate the longitudinal flow of propellant is presented. The instantaneous RT growth rate is derived at any point of adverse curvature along the flow, due to the combined effects of plasma pressure and propellant flow. Then it is converted to a practical expression in terms of specific impulse. In the course of the derivation, it will become evident that the growth rates from the two effects acting separately actually represent two distinct instabilities.

Finally, finite gyroradius stabilization due to collisionless ion gyroviscosity is used to estimate the shortest azimuthal wavelengths that are RT unstable. Their growth rates are compared with axial transit times through hypothetical regions of adverse curvature. On the basis of these calculations, it is recommended that the magnetic nozzle be (selfconsistently) shaped so as to have no regions of adverse magnetic curvature, to the extent possible.

In the following presentation, we do not attempt to consider longitudinal global effects on the structure and growth rates of flute instabilities, which would be induced by finitelength regions of adverse curvature. The treatment of such effects actually constitutes the subject of ballooning modes, which is sufficiently complicated as to deserve its own special presentation. The subject of ballooning modes in longitudinally flowing plasma therefore is beyond the purview of the present report. However, such work was carried out within the quest for magnetic fusion energy by Nagornyj, Ryutov, and Stupakov (Ref. 36) for plasmas confined longitudinally by magnetic mirrors. That configuration is closely related to the magnetic nozzle concept. They found that ballooning instabilities originating in regions of adverse curvature could be stabilized by the presence of nearby regions of strong good curvature, if such regions were sufficiently loaded with plasma. (The nozzle throat and the mirror 
throat are regions of good curvature.) Even so, they found that there remained residual instabilities. The results of Nagornyj, Ryutov, and Stupakov are not easily utilized in a practical manner for the problem of plasma flow in magnetic nozzles. That work ought to be revisited within the present context. Of course the entire problem could be avoided by eliminating regions of adverse curvature. Due to the selfconsistent modification of the vacuum magnetic nozzle field by the injection of hot plasma, this approach seems to imply that the nozzle be rather long and gradual.

\subsubsection{Surface Instability of Static Plasma in Region of Adverse Curvature}

We consider a static field-free plasma configuration of uniform pressure $P_{\text {tot }}$ and mass density $\rho$, confined by the pressure of external magnetic field $\mathbf{B}, B^{2} / 2 \mu_{0}$, in a region of equilibrium adverse curvature of radius $R_{\text {eq }}$ (the center of curvature lies within the plasma). This is illustrated in Figure 5. The plasma pressure and magnetic pressure is acting on the rim element of width $\mathrm{d} r\left(<<R_{\text {eq }}\right)$, subtending a very small meridian angle $d \phi$. Those radial forces must sum to zero in equilibrium. The propellant velocity flow vector is V. Centrifugal force density (not shown) is outward, with magnitude $\rho V^{2} / R$, and $R \approx R_{\text {eq. }}$. Outward radial displacement $\xi$ of the rim element as a whole (with $\mathrm{d} R$ and $\mathrm{d} \phi$ held fixed) is monitored for instability growth rate.

The forces are the pressures multiplied by their respective surface areas. In calculating the various surface areas of the rim element, the element is assumed to extend an azimuthal distance $d y$ perpendicular to the plane of Figure 5. For brevity, $\mathrm{d} y$ will be suppressed in the equations. For example, the surface area associated with the outer arc of the rim element in equilibrium is just written as $R_{\mathrm{eq}} \mathrm{d} \phi$, instead of $R_{\mathrm{eq}} \mathrm{d} \phi \mathrm{d} y$.

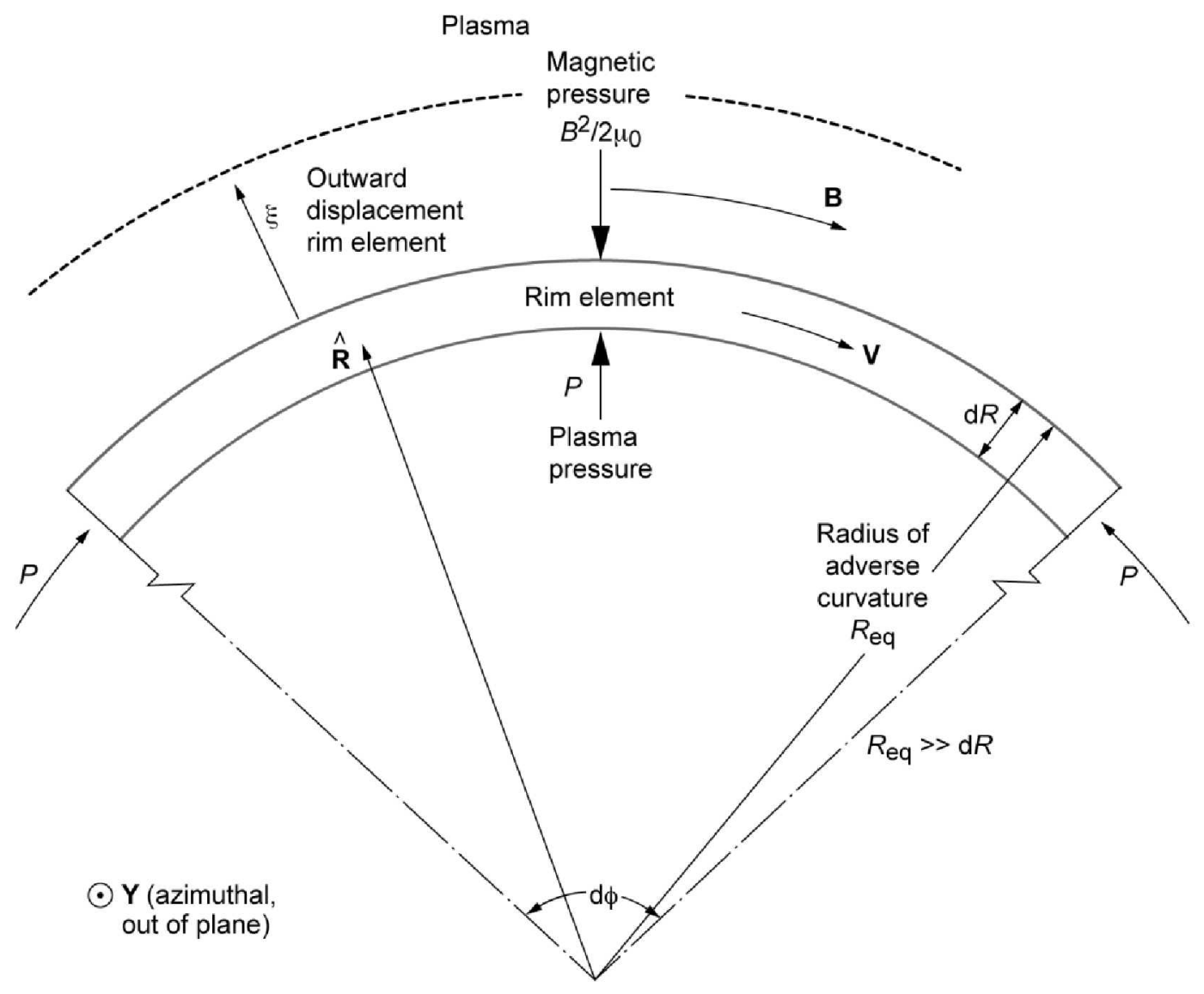

Figure 5.-Side-on view of plasma in region of adverse curvature. 
The elongated flute mode analyzed here is energetically favorable because, unlike oblique modes, it does not bend field lines. Also, it is basically a surface perturbation, which has only a limited influence beneath the surface. The width of the rim element in Figure 5, $\mathrm{d} R$, is chosen to encompass the range of influence under the surface. Farther below the surface than $\mathrm{d} R$, the fluid remains practically undisturbed by the presence of the surface perturbation. The specific interpretation of $\mathrm{d} R$ will be made clear below.

In terms of Figure 5, radial forces are also vertical forces, to lowest order in small $\mathrm{d} \phi$. Therefore, in equilibrium, the total radial force $\mathrm{d} F_{R}$ acting vertically on the element as a whole (see Fig. 5), to lowest order in $d \phi$, then reads

$$
\begin{aligned}
\mathrm{d} F_{R} & =P_{\text {tot }}\left(R_{\text {eq }}-\mathrm{d} R\right) \mathrm{d} \phi+P_{\text {tot }} \mathrm{d} R d \phi-\left(\frac{B_{\text {eq }}^{2}}{2 \mu_{0}}\right) R_{\text {eq }} \mathrm{d} \phi \\
& =0
\end{aligned}
$$

in mks units. The first term in Equation (208) is the outward radial (vertical) force acting on the inner arc (i.e., inner surface) of the rim element. The second is the radial (vertical) force due to lateral pressure acting on the canted end surfaces of the element. The effective radial component, or vertical component, of lateral pressure acting on each end of the rim element, which is experienced by the rim element as a whole, is just $P_{\text {tot }} \times[1 / 2 \mathrm{~d} \phi]$; the surface area of each end is $\mathrm{d} R$ (see Fig. 5). The third term in Equation (208) is the inward radial (vertical) force due to external magnetic pressure on the outer arc (outer surface) of the rim element. The notation used is that $B$ at radius $R=R_{\text {eq }}$ is denoted as $B_{\text {eq }}$.

After canceling some terms in Equation (208), the equilibrium condition reduces to

$$
P_{\text {tot }}=\frac{B_{\mathrm{eq}}^{2}}{2 \mu_{0}}
$$

This condition will be utilized in the following discussion of the dynamical behavior of the rim element as it undergoes a slight displacement.

Now consider the rim element as being displaced outward radially by a small distance $\xi$ (see Fig. 5). Both the inner and outer arcs are displaced outward by distance $\xi$, while keeping a fixed separation $\mathrm{d} R$ and holding fixed the angle $\mathrm{d} \phi$. The plasma fluid both upstream and downstream of the considered rim element is likewise displaced in the meridional plane, in the elongated flute mode being discussed. In this connection, it is important to note that the external magnetic field in the vacuum region, into which the displaced plasma will protrude, is completely determined in the local neighborhood. In particular, its $R$-dependence is determined.
The local dependence of external $B$ on $R$ can be demonstrated with the aid of a stream function $\Psi$, with $\mathbf{B}=\nabla \times(\hat{\mathbf{y}} \Psi)$ $=\hat{\mathbf{y}} \times \nabla \psi$, and $\nabla^{2} \psi=0$ from $\nabla \times \mathbf{B}=0$ in vacuum. Here, the symbol $\nabla^{2}$ represents the two-dimensional Laplacian operator in the plane of Figure 5, and $\hat{\mathbf{y}}$ is a locally constant unit azimuthal vector out of that plane. The Laplacian operator is expressed in local cylindrical coordinates based upon the local radius of curvature, and the boundary condition on $\mathbf{B}$ is applied at the equilibrium plasma surface. The salient feature of the vacuum magnetic field just above the plasma surface is that it depends locally on the radius (for small $\xi$ ) as $1 / R$, with $R=R_{\text {eq }}+\xi$. It is important to note that the linearly small amplitude $\xi$ is much smaller than the flute mode's azimuthal wavelength out of the plane of Figure 5.

Concomitantly, the external magnetic flux is not constrained to accumulate over the plasma's outward-moving conducting protrusion, because the protrusion actually is of limited extent in azimuth (perpendicular to the plane of Fig. 5). The external longitudinal magnetic field lines are shunted azimuthally to either side of the vertically extended conducting surface instead of piling up on top of it, thereby maintaining a state of lower magnetic energy. That is why the external magnetic field magnitude $B$, just above the crest of the protrusion, can be taken as being undistorted by the presence of the flute mode.

Consequently, the field magnitude $B$ just above the crest of the protrusion is related to the field $B_{\text {eq }}$ at the equilibrium surface by

$$
\frac{B}{B_{\text {eq }}}=\frac{R_{\text {eq }}}{R_{\text {eq }}+\xi}
$$

The local spatial behavior of the longitudinal magnetic field just outside of the plasma constitutes the principal reason for the adverse curvature instability. As displayed in Equation (210), the field strength becomes locally weaker going away from the plasma, in the region of adverse curvature. Consequently, a plasma element that is displaced outwards is subjected to a smaller magnetic backpressure. Thus, the plasma displacement continues to grow outwards.

This critical behavior of the spatial variation of the longitudinal magnetic nozzle field can be directly exhibited by utilizing Ampere's law, which is $\nabla \times \mathbf{B}=\mu_{0} \mathbf{J}$ in mks units. By means of Stokes's theorem, Ampere's law can be converted to an integral representation, which reads $\oint \mathbf{B} \cdot \mathrm{d} \mathbf{l}=\mu_{0} I$. Here, the indicated loop integral is taken around a closed contour, with $I$ being the net current through the contour. In the case being considered, the contour is around a wedge-shaped rectangle similar to that shown in Figure 5. In the present situation, however, the inner leg of 
the rectangle lies just below the perfectly conducting sharp boundary, where $B=0$. The outer leg of the rectangle lies a displacement distance $\xi$ above the boundary, say at $R=R_{\mathrm{eq}}$ $+\xi$, in the notation of Figure 5. Then the integral form of Ampere's law reduces exactly to $B R \mathrm{~d} \phi=\mu_{0} K R_{\text {eq }} \mathrm{d} \phi$, where $K$ is the azimuthal surface current density on the boundary. Thus, the field strength just beyond the boundary is $B=$ $\mu_{0} K R_{\text {eq }} / R$, which is exactly equivalent to Equation (210).

Although an equilibrium boundary was assumed in deriving Equation (210), the identical result for $B(R)$ is also obtained when taking into account the presence of flute deformations of the boundary. This result will be shown explicitly in Appendix K.

Our simplified physical derivation of the RT type of instability adapts the viewpoint of a plasma element defined in the meridional $r, Z$-plane. The forces operating on the plasma element are identified and summed within this plane. This approach highlights the role of adverse longitudinal curvature, which clearly must influence a mode that is extended along the magnetic field (flute mode).

On the other hand, we suppress physical effects from cylindrical geometry associated with curvature the short way around. This approximate treatment is valid for small azimuthal wavelengths. By looking downstream at the r, $\theta$-plane, one sees that a very localized (in azimuth) flute-mode crest can scarcely be "aware" of the cylindrical curvature. The only reminder of cylindrical geometry is that there be an integer number of wavelengths around the circumference, to avoid discontinuous behavior. This integer is the so-called azimuthal mode number $M_{\theta}$, and the associated azimuthal wave number is then

$$
k=M_{\theta} / r
$$

where $r$ is the cylindrical radius.

Knowing the radial dependence of the external magnetic field allows us to write the equation of motion of the rim element in terms of its displacement $\xi$. The equation of motion reads

$$
\left(\rho R_{\text {eq }} \mathrm{d} R \mathrm{~d} \phi\right)\left(\frac{\mathrm{d}^{2} \xi}{\mathrm{d} t^{2}}\right)=\mathrm{d} F_{R}
$$

Here, $\rho R_{\text {eq }} \mathrm{d} R \mathrm{~d} \phi$ is the mass of the rim element (suppressing $\mathrm{d} \theta$ ), to zero order in $\xi$. Higher order corrections in $\xi$ to this mass element are not relevant in a linear theory for small $\xi$ because the acceleration in Equation (212), $\mathrm{d}^{2} \xi / \mathrm{d} t^{2}$, is already first order in $\xi$. Similarly, on the right side of Equation (212), the total radial (vertical) force on the displaced rim element, $\mathrm{d} F_{R}(\xi)$, needs to be computed only to first order in $\xi$. That force is

$$
\begin{aligned}
& \mathrm{d} F_{R}(\xi)=P_{\text {tot }}\left(R_{\text {eq }}+\xi-\mathrm{d} R\right) \mathrm{d} \phi+P_{\text {tot }} \mathrm{d} R \mathrm{~d} \phi \\
&-\frac{B_{\text {eq }}^{2}}{2 \mu_{0}} \frac{R_{\text {eq }}^{2}}{\left(R_{\text {eq }}+\xi\right)^{2}}\left(R_{\text {eq }}+\xi\right) \mathrm{d} \phi
\end{aligned}
$$

The first term in Equation (213) is the outward force on the displaced inner arc (surface) of the rim element. The second term is the outward force on the rim element as a whole due to the lateral pressure at its canted ends (still of radial width $\mathrm{d} R$ and subtending angle $\mathrm{d} \phi$ ). The last term is the inward magnetic force on the displaced outer arc. Equation (210) has been used.

Actually, the effective lateral pressure (averaged over $\mathrm{d} R$ ) is smaller than indicated in Equation (213), second term. Continuity of pressure at the displaced outer surface (to avoid unphysically large acceleration of the outer surface) requires that the internal plasma pressure should spatially decrease so as to approach the reduced external magnetic pressure there. Taking this feature into account, however, only proves to introduce a small correction of order $\mathrm{d} R / R_{\mathrm{eq}}$. This remark will be quantified below.

After some cancellations in Equation (213), and expanding to first order in $\xi$ and invoking the equilibrium condition, Equation (209), the force in Equation (213) becomes simply

$$
\mathrm{d} F_{R}(\xi)=2 P_{\text {tot }} \xi \mathrm{d} \phi
$$

We substitute the first-order force equation, Equation (214), into the equation of motion, Equation (212) and look for a temporally growing displacement $\mathrm{d} \xi / \mathrm{d} t=\gamma_{\mathrm{RT}} \xi$ having RT growth rate $\gamma_{\mathrm{RT}}$. After further cancellations, the equation of motion reduces to an expression for $\gamma_{\mathrm{RT}}$ :

$$
\gamma_{\mathrm{RT}}^{2}=\frac{2 P_{\text {tot }} / \rho}{R_{\text {eq }} \mathrm{d} R}
$$

At this point, it is important to recognize that the most energetically favorable motions of plasma in producing the flute instability are those that do no compressive work; that is, the internal fluid motions in the plane perpendicular to B are incompressible. For such incompressible motions, a flute mode at the plasma surface with the azimuthal dependence $e^{i k y}$ has only a limited influence beneath the surface, with the radial dependence $e^{-k x}$, where $x(>0)$ is the distance beneath the surface (e.g., Ref. 37). To derive this feature, write the fluid momentum equation for small perturbations in the plane perpendicular to $\mathbf{B}$ as $\rho \gamma_{\mathrm{RT}}\{\delta \mathrm{V}\}+\nabla\left\{\delta P_{\text {tot }}\right\}=0$. Then the condition of incompressibility $\nabla \cdot\{\delta \mathbf{V}\}=0$ implies that $\nabla^{2}\left\{\delta P_{\text {tot }}\right\}=0$. Inserting the azimuthal mode dependence $e^{i k y}$ into Laplace's equation for the pressure perturbation $\delta P_{\text {tot }}$ confirms the stated result. 
Therefore, the effective depth of the rim element influenced by the surface mode is $\mathrm{d} R=1 / k$, with $k$ being the azimuthal wave number. Plasma fluid at lower depths is practically undisturbed by flute-mode perturbations at the plasma surface. Thus, we interpret $\mathrm{d} R$ as $1 / k$ in Equation (215). The RT-type growth rate then becomes

$$
\gamma_{\mathrm{RT}}=\sqrt{\frac{2 P_{\text {tot }} / \rho}{R_{\mathrm{eq}}} k}
$$

Note that $2 P_{\text {tot }} / \rho \approx S^{2}$, where $S$ is the speed of sound in the plasma. Equation (216) thereby has the form of a gravitational instability of the RT type; namely $\gamma_{\mathrm{RT}}=\sqrt{g k}$, in which $g \approx S^{2} / R_{\mathrm{eq}}$. The effective gravitational acceleration $g$ might therefore be interpreted as the centrifugal force of charged particles (of small gyroradius) that move approximately with thermal velocity in either direction along the curved magnetic field at the plasma surface. Nevertheless, this flute mode is not a true gravitational instability because no effective gravitational field permeates the plasma.

To check the assumption of incompressible motion, note that the plasma's velocity $\mathrm{d} \xi / \mathrm{d} t=\gamma_{\mathrm{RT}} \xi$, as well as the effective wave velocity $\gamma_{\mathrm{RT}} / k$, should both be small compared to the speed of sound $S$. The first condition is satisfied because $\xi$ is arbitrarily small. The second condition reduces to $k R_{\text {eq }} \gg 1$. The azimuthal wave number is $k=M_{\theta} / r$ (Eq. (211)), where $r$ (distinct from $R_{\mathrm{eq}}$ ) is the cylindrical radius of the propellant, and $M_{\theta}$ is the azimuthal mode number (i.e., $e^{i k y}=e^{i M_{\theta} \theta}$ ). Therefore, the second condition for incompressible motion is satisfied whenever the radius of curvature $R_{\mathrm{eq}}$ is large compared with $r$, the propellant's radius, or when $M_{\theta} \gg 1$.

Finally, we return to the requirement of continuity of pressure at the outer displaced surface of the rim element. The above model, taken literally, yields the following expression for the fractional discontinuity of pressure there (we omit the details):

$$
\left[\left.\frac{P_{\text {tot }}-\left(B^{2} / 2 \mu_{0}\right)}{P_{\text {tot }}}\right|_{\rfloor_{R=R_{\mathrm{eq}}+\xi}}=\left(\frac{\xi}{\mathrm{d} R}\right)\left(\frac{\mathrm{d} R}{R_{\mathrm{eq}}}\right) \ll 1\right.
$$

expanded to first order in $\xi$ and using the equilibrium relation, Equation (209). Since $\mathrm{d} R$ is comparable to an azimuthal wavelength, linear theory for small amplitude motions requires that $\xi \ll<\mathrm{d} R$. Hence, the first factor on the right has to be small in the linearized dynamical model. The second factor is also small, assuming that azimuthal wavelengths of the considered flute modes are small compared to the radius of curvature of the magnetic nozzle field. Consequently, in this limit, the error in continuity of pressure at the displaced interface is negligible.

\subsubsection{Surface Instability of Flowing Propellant in Region of Adverse Curvature}

The previous example serves to illustrate the methods to be applied to flowing propellant in the magnetic nozzle. It will be assumed in this section that the external magnetic field diffuses only a small distance into the plasma compared with the thickness of the rim element. The previously derived pressure-driven contribution to the growth rate persists, but proves to be augmented by the centrifugal force effect of the curved flow. The practical expression for the growth rate, derived below, would be useful offline, in postprocessing of axially symmetric resistive MHD simulations of magnetic nozzle flow. In this manner one could follow the flow and the self-consistent nozzle geometry and thereby estimate the extent of e-folding of flute instabilities within regions of adverse curvature, without the necessity of running three-dimensional simulations.

The centrifugal force density due to propellant flow with velocity $V_{/ /}$is here denoted by $F_{g}$ and is locally defined as

$$
F_{g}=\frac{\rho V_{\| \prime}^{2}}{R}
$$

where $\rho$ is propellant mass density, and radius $R\left(\approx R_{\mathrm{eq}}\right)$ lies within the rim element depicted in Figure 5 . This is equivalent to gravitational force density $\rho g$ with $g=V_{/ /}^{2} / R$.

In this case, there are two relevant equilibrium conditions: a local one and a global one. Local internal equilibrium becomes nonuniform, due to the effective gravitational field that now permeates the plasma. The condition of internal equilibrium is

$$
\partial_{R} P_{\text {tot }}=F_{g}
$$

Here, as usual, $P_{\text {tot }}$ is the local, total plasma pressure due to both electrons and ions.

The condition Equation (219) holds not only for the equilibrium configuration, but also for the time-dependent perturbed state with growing displacement $\xi$. As long as the radial transit time for sound propagation through depth $\mathrm{d} R$ is short compared to the growth time of the mode, Equation (219) holds. The latter condition ultimately can be expressed as $\gamma_{\mathrm{RT}} / k \ll S$, which is the same as the condition for incompressible motion mentioned in the previous example.

We turn now to the condition of global equilibrium, which applies to the rim element as a whole. The notation $P_{0}$ refers to plasma pressure at the inner arc of the element, and 
$P_{1 / 2} \approx P_{0}+1 / 2 \mathrm{~d} R \partial_{R} P_{\text {tot }}$ represents pressure halfway up on the width $\mathrm{d} R$ and is representative of the average lateral pressure on $\mathrm{d} R$. The centrifugal force density $F_{g}$ will be regarded as an average value within the volume of the rim element. Then, equilibrium force balance on the rim element of Figure 5, radially (or vertically), reads

$$
\begin{aligned}
& P_{0}\left(R_{\mathrm{eq}}-\mathrm{d} R\right) \mathrm{d} \phi+P_{1 / 2} \mathrm{~d} R \mathrm{~d} \phi \\
& \quad+F_{\mathrm{g}}\left(R_{\mathrm{eq}}-\frac{1}{2} \mathrm{~d} R\right) \mathrm{d} R \mathrm{~d} \phi=\left(\frac{B_{\mathrm{eq}}^{2}}{2 \mu_{0}}\right)_{\rfloor_{R=R_{\mathrm{eq}}}} R_{\mathrm{eq}} \mathrm{d} \phi
\end{aligned}
$$

The first term in Equation (220) is the outward force acting on the lower surface of the rim element, and the second term is the outward force on the element due to lateral pressure acting on its canted ends. The third term, analogous to the effect of gravity on the element, is the outward force due to the centrifugal force density $F_{g}$ multiplied by the volume of the element $\left(R_{\mathrm{eq}}-1 / 2 \mathrm{~d} R\right) \mathrm{d} R \mathrm{~d} \phi$. An average (midvalue) has been used for the arc-length in the volume element. The right side of Equation (220) constitutes the inward force acting on the element due to the external magnetic pressure acting on its outer surface. Dividing through by $R_{\text {eq }} \mathrm{d} \phi$ using $P_{1 / 2}=P_{0}+1 / 2 \mathrm{~d} R \partial_{R} P_{\text {tot }}$ and using Equation (219), Equation (220) then can be written exactly as

$$
P_{0}+F_{g} \mathrm{~d} R=\left(\frac{B_{\mathrm{eq}}^{2}}{2 \mu_{0}}\right)
$$

Equation (221) is the consequence of global equilibrium of the rim element in Figure 5. It is as if $\left(F_{g} \mathrm{~d} R\right)=\partial_{R} P_{\text {tot }} \mathrm{d} R$ is the pressure increment to be added to the inner pressure $P_{0}$ to ensure pressure continuity across the outer surface of the rim element.

The linear extrapolation of pressure within $\mathrm{d} R$, $P_{1 / 2}=P_{0}+1 / 2 \mathrm{~d} R \partial_{R} P_{\text {tot }}$, assumes that the first two terms in the Taylor series for the pressure profile are sufficient; that is, the pressure gradient term is assumed to constitute a small correction. This formula, used to obtain Equation (221), proves to require the strong inequality $\left(\mathrm{d} R / R_{\mathrm{eq}}\right) V^{2} \ll S^{2}$ (refer to Eq. (219)). Because a large radius of curvature is assumed in the sense $\mathrm{d} R \ll R_{\text {eq }}$, it follows that the equilibrium model allows transonic and supersonic flows within $\mathrm{d} R$.

Let us define $\mathrm{d} R=1 / k$ for the flute-mode wave number. Since $k=M_{\theta} / r$ (Eq. (211)), the above inequality then can be put in the form $\left(V^{2} / S^{2}\right) \ll M_{\theta} R_{\mathrm{eq}} / r$. In this form, transonic and supersonic flows can be handled within the dynamical model. The required geometric conditions are that the radius of curvature $R_{\mathrm{eq}}$ be large compared with the cylindrical radius $r$ or that the azimuthal mode number $M_{\theta}$ is large compared to 1 . The same linear extrapolation of the pressure profile (with its same consequence) is relevant to the next problem.

We turn now to the dynamical problem, which is to calculate the net force on the plasma element as it is displaced outwards by the amount $\xi$, as in the previous example. As before, that force will be inserted into the equation of motion (Eq. (212)) of the element, so as to obtain the growth rate of the instability. Superscript $\xi$ will refer to the state of the superscripted quantity as it exists in the displaced element; for example, $P_{0}^{\xi}$ refers to the pressure on the displaced inner surface of the rim element, and $P_{1 / 2}^{\xi}$ refers to the pressure halfway up on $\mathrm{d} r$ in the displaced element.

The force on the rim element is written as

$$
\begin{aligned}
\mathrm{d} F_{R}=P_{0}^{\xi}\left(R_{\mathrm{eq}}-\mathrm{d} R\right. & +\xi) \mathrm{d} \phi+P_{1 / 2}^{\xi} \mathrm{d} R \mathrm{~d} \phi \\
& +F_{g}^{\xi}\left(R_{\mathrm{eq}}-1 / 2 \mathrm{~d} R+\xi\right) \mathrm{d} R \mathrm{~d} \phi \\
& -\left(\frac{B_{\mathrm{eq}}^{2}}{2 \mu_{0}}\right) \frac{R_{\mathrm{eq}}^{2}}{\left(R_{\mathrm{eq}}+\xi\right)^{2}}\left(R_{\mathrm{eq}}+\xi\right) \mathrm{d} \phi
\end{aligned}
$$

Here, the first term is the outward pressure-force on the displaced lower surface, and the second term is the outward force on the element from lateral pressures at the canted ends (still of width $\mathrm{d} R$ and subtending angle $\mathrm{d} \phi$ ). The third term is the outward gravitational force density multiplied by the displaced volume using its average arc length. Finally, the last term is the inward magnetic-pressure force on the displaced outer surface of the element. The $1 / R$ dependence of the external magnetic field, with $R=R_{\text {eq }}+\xi$, has been invoked.

The above-mentioned linear extrapolation within $\mathrm{d} R$ for $P_{1 / 2}^{\xi}$, and the local equilibrium relation Equation (219) are used, as well as the global equilibrium Equation (221) as it regards $B_{\mathrm{eq}}^{2}$. Expanding expressions to first order in $\xi$, Equation (222) then becomes

$$
\mathrm{d} F_{R}=R_{\text {eq }} \mathrm{d} \phi\left[\begin{array}{l}
\left(P_{0}^{\xi}-P_{0}\right)+\left(F_{g}^{\xi}-F_{g}\right) \mathrm{d} R \\
+\frac{\xi}{R_{\text {eq }}}\left(P_{0}^{\xi}+F_{g}^{\xi} \mathrm{d} R+P_{0}+F_{g} \mathrm{~d} R\right)
\end{array}\right]
$$

To first order in $\xi$, the final square bracket in Equation (223) may as well be written $2\left(P_{0}+F_{g} \mathrm{~d} R\right)$.

The first term on the right in Equation (223) can be written as $\xi \partial_{R} P_{\text {tot }}\left(=\xi F_{g}\right)$. The physical reason for being able to 
write it this way is that the displaced fluid element climbs up the preexisting equilibrium pressure profile, because rising fluid at the displaced lower surface of the element is replaced at that level by azimuthal inflow of fluid from either side. The replacement fluid brings with it the original equilibrium pressure-profile at that level. (Note that the fluid below $\mathrm{d} R$ remains practically undisturbed.)

The second term on the right of Equation (223), due to the rising fluid, involves spatial variation of density $\rho$ and position $R$ (supposing zero-order flow velocity $V_{/ /}$is constant). It can be written as $\xi \partial_{R} F_{g} \mathrm{~d} R\left(=\xi F_{g} \mathrm{~d} R\left(L_{\rho}^{-1}-R^{-1}\right)\right)$. Here, $L_{\rho}^{-1}=\rho^{-1} \partial_{R} \rho$ is the inverse scale length for radial variation of propellant density in the core plasma (not in the edge layer). The core plasma is assumed to be fairly uniform, with $L_{\mathrm{\rho}} \sim R \gg \mathrm{d} R$. Then the entire second term, on the order of $\xi F_{g} \mathrm{~d} R / R$, can be neglected compared with the first term, $\xi F_{g}$.

The third term is $2\left(P_{0}+F_{g} \mathrm{~d} R\right)\left(\xi / R_{\text {eq }}\right)$. The gravitational contribution (from $F_{g}$ ) is again small compared with the first term in Equation (223) because $\mathrm{d} R<<R_{\text {eq. }}$.

Therefore, neglecting terms on the order of $\mathrm{d} R / R_{\text {eq }}$ in Equation (223) compared to the first term, Equation (223) can be written simply as

$$
\left.\mathrm{d} F_{R}=R_{\mathrm{eq}} \mathrm{d} \phi\left\{\xi \xi F_{g}+2 P_{0} \frac{\xi}{R_{\mathrm{eq}}}\right\}\right\}
$$

Using this force in the equation of motion (Eq. (212)) of the rim element together with the temporally growing representation $\mathrm{d} \xi / \mathrm{d} t=\gamma_{\mathrm{RT}} \xi$ yields the following result for $\gamma_{\mathrm{RT}}$ :

$$
\gamma_{\mathrm{RT}}^{2}=\frac{F_{\mathrm{g}}+2 P_{\mathrm{tot}} / R_{\mathrm{eq}}}{\rho \mathrm{d} R}=\frac{\left(V_{/ /}^{2} / R_{\mathrm{eq}}\right)+\left(2 P_{\mathrm{tot}} / \rho R_{\mathrm{eq}}\right)}{\mathrm{d} R}
$$

Here, $V_{/ /}$refers to the velocity of propellant flow parallel to the magnetic field $\mathbf{B}$. It does not really matter in Equation (225) whether $P_{0}$ or $P_{\text {tot }}$ is used anywhere in $\mathrm{d} R$, since the difference is small, on the order of $\mathrm{d} R / R$ compared with $F_{\boldsymbol{g}}$

The previous discussion about incompressible perturbation flow in flute-mode dynamics (in the plane perpendicular to $\mathbf{B}$ as before) provided that the plasma properties $\left(\rho, P_{\text {tot }}\right.$, and $V_{/ l}$ ) vary only weakly within $\mathrm{d} R$. Then, the periodic azimuthal mode structure $e^{i k y}$ again implies that the radial mode structure $e^{-k x}$ decreases exponentially beneath the surface. Thus, we again have the relation $\mathrm{d} R=k^{-1}$ in terms of the azimuthal wave number $k$. The growth rate, which now includes the effects of both flow and pressure in the adverse-curvature region, finally can be written as follows:

$$
\gamma_{\mathrm{RT}}=\sqrt{\left(\frac{V_{l /}^{2}+2 P_{\mathrm{tot}} / \rho}{R_{\mathrm{eq}}}\right) k}
$$

The effective gravitational accelerations in regions of adverse curvature, which arise both from thermal motions of particles and from propellant flow, just appear in an additive manner within the growth rate. This is what would be expected intuitively. Bidirectional diffusion of plasma and magnetic field across the fluted surface will then create a plasma-field mixing layer.

As before in the pressure-driven case, the model used to derive this growth rate suffers from a pressure discontinuity at the outer displaced surface of the plasma. Nevertheless, it can again be demonstrated that the fractional pressure discontinuity reduces to $(\xi / \mathrm{d} R)\left(\mathrm{d} R / R_{\mathrm{eq}}\right)$, which is very small for the two conditions: linearly small amplitudes $\xi \ll \mathrm{d} r$, and $\mathrm{d} r \ll R_{\text {eq. }}$. We shall omit the details of the demonstration.

The purpose of this section is to provide rough guidance as to whether the idealized RT instability has a chance to grow. $L_{\text {arc }}$ is the complete length of arc in the adverse curvature region at the plasma edge (convex outwards), and is not necessarily small. Concomitantly the subtended longitudinal angle in radians is $\delta \phi=L_{\text {arc }} / R_{\text {eq }}$. This finite longitudinal angle is to be distinguished from the "small" longitudinal angle used in the simplified model, d $\phi$. Nevertheless, as a simplification, we shall assume that the growth rate, Equation (226), is still valid. Part of this growth rate contains the expression $2 P / \rho$, but the speed of sound is $S^{2}=\gamma P / \rho$, and we shall take $\gamma=5 / 3$. Then, $2 P / \rho=\frac{6}{5} S^{2}$. The condition for growth is $\gamma_{\mathrm{RT}}^{2} t_{\mathrm{cur}}^{2}>1$, with $t_{\text {cur }}$ denoting the time the edge plasma spends in the adverse curvature region. This time is $t_{\text {cur }}=L_{\text {arc }} / V_{/ /}$. We use Equation (226) in the growth condition $\gamma_{\mathrm{RT}}^{2} t_{\text {cur }}^{2}>1$, but with the azimuthal wave number $k$ replaced by $M_{\theta} / r$, where $M_{\theta}$ is the number of azimuthal wavelengths that fit into the circumference, $2 \pi r$, the short way around. Then, the condition for growth can be expressed as

$$
(\delta \phi) \frac{M_{\theta} L_{\mathrm{arc}}}{r}>\left[1+\frac{6}{5} \frac{S^{2}}{V_{/ /}^{2}}\right]^{-1}
$$

The quantities $S^{2}$ and $V_{/ /}^{2}$ should be taken as average values along $L_{\text {arc }}$, which can be determined from axi-symmetric ideal MHD simulations. Ion magneto-viscosity sets an upper 
limit to $M_{\theta}$ (see Eq. (234)). Except for a complete treatment, the Hall effect (which is destabilizing) should also be included. This is examined in Section 4.3, "Finite Larmor Radius (FLR) Stabilization of RT-Type Flute Modes," and in Section 4.3.1, "Example: Estimate of Hall Term in Coaxial MHD Thruster."

Equation (226) can be converted to a practical formula for the growth rate that is characterized by $V_{\text {ex }}$, the velocity of propellant at the nozzle's exit plane (the specific impulse is given by $V_{\mathrm{ex}} \mathrm{m} / \mathrm{s} \div 9.8 \mathrm{~m} / \mathrm{s}^{2}$ ). The practical formula could be utilized as a postprocessing tool with axially symmetric MHD simulations of propellant flow in magnetic nozzles.

To derive the practical formula, note that the local speed of sound, squared, is given by $S^{2}=\gamma P_{\text {tot }} / \rho$. Then, by means of the Bernoulli equation with the choking condition at the throat, using the adiabatic relations along the longitudinal flow, and using Equation (211), Equation (226) can be written as

$$
\gamma_{\mathrm{RT}}=V_{\mathrm{ex}} \sqrt{\left[1-\left.\frac{3}{5}\left(\frac{\rho}{\rho_{b}}\right)^{2 / 3}\right|_{]}\left(\frac{M_{\theta}}{R_{\mathrm{eq}} r}\right)\right.}
$$

The adiabatic index (ratio of specific heats) is taken to be $\gamma=5 / 3$ (i.e., there are occasional collisions), and relations based upon infinite contraction and expansion ratios in the nozzle geometry are utilized. However, the Bernoulli flow solutions are such that the results are hardly changed by using realistic values such as 2 for radius contraction and 3 for radius expansion.

Mass density $\rho$ varies along the flow, starting with its breech value $\rho_{b}$ and finally vanishing at the exit plane. After taking the square root in Equation (228), the numerical factor due to this density variation ranges from 0.63 in the breech to 1.0 at the exit. Therefore, the density expression can be omitted by instead inserting an average numerical factor of 0.8 , which is within $\approx 30$ percent of the exact numerical value at any point along the flow. Then Equation (228) reads

$$
\gamma_{\mathrm{RT}} \approx 0.8 V_{\mathrm{ex}} \sqrt{\left(\frac{M_{\theta}}{R_{\mathrm{eq}} r}\right)}
$$

The radius of curvature $R_{\mathrm{eq}}$ and the cylindrical radius of propellant $r$ also vary along the flow, as could be monitored in MHD simulations. The $R_{\mathrm{eq}}$ is interpreted as positive in regions of adverse curvature and negative in regions of good curvature. In the latter case, the RT-type flute mode is a gravity wave that just oscillates without growing. The azimuthal mode number $M_{\theta}$ remains to be discussed.

The final issue of interest here, for macroscopic RT-type instabilities, involves the maximum azimuthal mode number
$M_{\theta}$ to be used in the expression for the growth rate in Equations (228) or (229). Arbitrarily large mode numbers would produce arbitrarily large growth rates, within the context of the present sharp-boundary model of the propellant. The question as to the maximum effective mode number can be answered in terms of FLR stabilization of the RT-type flute modes. For the hot plasmas $(\sim 100 \mathrm{eV})$ contemplated for magnetic nozzle applications in space vehicle propulsion, FLR stabilization can be a noticeable effect.

\subsection{Finite Larmor Radius (FLR) Stabilization of RT-Type Flute Modes}

In the preceding section, we explored plasma particle trajectories at their initial impact with the confining magnetic field. The concern was brought up that their sharp adverse longitudinal curvatures could potentially be a source of instabilities in the injection region. In the following sections, on the other hand, we shall look at adverse longitudinal curvature instabilities further downstream, wherein a steady flow along the confining magnetic field has already been established at the plasma boundary.

In the plane perpendicular to magnetic field $\mathbf{B}$, it is well known from plasma transport theory that ions within the plasma-field mixing layer would possess a collisionless gyroviscosity when the plasma is of sufficiently high temperature that ion collisions are infrequent (see Refs. 9 and 32). Even though there is no collisional dissipation as is usually connected to viscosity, the ion gyroviscosity can exert a stabilizing influence on RT flute instabilities by modifying the dynamical processes that have enabled the modes to grow. Moreover, the flute-mode amplitude is largest at the plasma-field interface with the magnetic field, decaying exponentially into the plasma. Therefore, the gyroviscosity is effective just where the mode amplitude is the largest.

Viscous diffusivity for ion gyroviscosity can be represented approximately as (Ref. 9)

$$
D_{\text {vis } i}=a_{i} V_{\text {th } i}
$$

where $a_{i}$ is the thermal ion gyroradius. This expression is analogous to the ordinary kinematic viscosity, $\lambda_{i} V_{\text {th }} i$, wherein $\lambda_{i}$ is the mean free path for ion-ion coulomb collisions. Kinematic viscosity is the viscosity coefficient divided by $n m_{i}$. A numerical coefficient of order 1 in Equation (230) proves to be almost irrelevant since it only enters the final expression for the growth rate as raised to the one-third power.

In Equation (230) $a_{i}=V_{\text {th } i} / \omega_{c i}$, the ion thermal velocity is $V_{\text {th } i}=\sqrt{2 T / m_{i}}$, and $\omega_{c i}=q B / m_{i}$ is the ion gyrofrequency within the plasma-field interface (using mks units, with $T$ in joules). The gyroviscosity obviously vanishes for very small $a_{i}$, which is associated with very large magnetic 
fields. It is important to realize that the flute perturbation possesses an inverse time scale associated with this gyrodiffusivity, which is $k^{2} D_{\text {vis } i}$, where $k=M_{\theta} / r$ is the azimuthal wave number. In the case of ordinary diffusivity, $k^{2} D_{\text {vis } i}$ would signify a dissipative decay rate of a macroscopic dynamical mode, due to ion-ion collisions.

For mode numbers sufficiently large that the gyrodiffusive inverse-time becomes comparable to the RT growth rate, those modes would be influenced by FLR stabilization.

$$
k^{2} D_{\mathrm{vis} i} \approx \gamma_{\mathrm{RT}}
$$

The azimuthal mode number corresponding to the condition in Equation (231) will be calculated and regarded as approximating the maximum mode number for viable growth of RT-type flute instabilities associated with adverse curvature of magnetic field lines.

For $T_{e}=T_{i}=T$, and $\gamma=5 / 3$, the square of the speed of sound in the breech can be expressed as $S_{b}^{2}=\gamma P_{b} / \rho_{b}=5 / 3 V_{b \text { th } i}^{2}$. The propellant's exit velocity can then be written

$$
V_{\mathrm{ex}}=\sqrt{3} S_{b}=\sqrt{5} V_{b \text { th } i}
$$

Although this relation strictly is for infinite contraction and expansion, it also is a good approximation for realistic converging-diverging nozzle shapes. Use of Equation (232) in Equation (228) for $\gamma_{R T}$ allows the solution to Equation (231) for the upper azimuthal mode number with the following result:

$$
M_{\theta} \approx 5^{1 / 3} \frac{r}{\left(R_{\mathrm{eq}} a_{i}^{2}\right)^{1 / 3}}\left(\frac{T_{b}}{T}\right)^{1 / 3}\left[1-\left.\frac{3}{5}\left(\frac{\rho}{\rho_{b}}\right)^{2 / 3}\right|_{\rfloor} ^{1 / 3}\right.
$$

Since this expression is nondimensional, any consistent set of units can now be used.

Increasing the radius of adverse curvature $R_{\mathrm{eq}}$ decreases the driving force for the flute instability and hence decreases the upper mode number that can go unstable in the presence of ion gyroviscosity. Increasing the ion gyroradius $a_{i}$ also enhances the spatial phase mixing of the mode, spoiling the growth dynamics, which likewise acts to decrease the upper mode number that can go unstable.

A lower bound for this upper limit on mode number is obtained by raising the temperature $T$ up to its breech value in Equation (233) and by raising the mass density $\rho$ up to its breech value. These approximations actually are fairly harmless between breech and throat because $T$ at the throat only drops to three-fourths of its breech value, and $\rho$ at the throat only drops to about two-thirds of its breech value. Then Equation (233) becomes approximately

$$
M_{\theta} \approx 1.5 \frac{r}{\left(R_{\mathrm{eq}} a_{i}^{2}\right)^{1 / 3}}
$$

Equation (234) may also be written in terms of the azimuthal wave number $k=M_{\theta} / r$ as

$$
k a_{i} \approx 1.5\left(\frac{a_{i}}{R_{\mathrm{eq}}}\right)^{1 / 3}
$$

For parameters of interest here, relevant examples are $a_{i} \approx 1 \mathrm{~cm}$ and $R_{\text {eq }} \approx 30 \mathrm{~cm}$, in which case $k a_{i} \approx 0.5$. In other words, in ordinary MHD $k a_{i}<1$, but in gyroviscous MHD FLR stabilization sets in about when $k a_{i}$ is on the order of 1 .

Returning to Equation (234), the gyroradius within the plasma-field mixing layer is expressed as $a_{i}^{2}=1 / 2 \beta c^{2} / \omega_{p i}^{2}$.

Here, $\beta$ is the local ratio of total plasma pressure of electrons and ions to magnetic pressure at a representative position within the plasma-field interface, and $\omega_{p} i$ is the ion plasma frequency at that representative position within the interface. Then setting the average density in the interface to half the density in the core plasma and reverting to the ion plasma frequency expressed in terms of the core density, Equation (234) becomes

$$
M_{\theta} \approx\left(\frac{1.5}{\beta^{1 / 3}}\right) \frac{r}{\left[R_{\mathrm{eq}}\left(c^{2} / \omega_{p i}^{2}\right)\right]^{1 / 3}}
$$

Since the value of $\beta$ is not precisely known, but is near 1 within the interface and appears only to the one-third power, the prefactor in this expression is ignored.

For hydrogen ion number density $10^{15} \mathrm{~cm}^{-3}, c / \omega_{p i}=1 \mathrm{~cm}$. Examples of other relevant parameters are $r=15 \mathrm{~cm}$ and $R_{\mathrm{eq}}$ $=30 \mathrm{~cm}$. From Equation (236) one sees that $M_{\theta} \approx 5$ is about the upper limit of flute-mode azimuthal numbers that can be unstable, with respect to avoiding FLR stabilization. In any event, the RT growth rate only depends on $\sqrt{M_{\theta}}$. Moreover, to the extent that the prefactor in Equation (236) can be ignored, the upper limit on flute-mode number is independent of temperature, depending just on local plasma number density in the core, local cylindrical radius of plasma, and local radius of curvature at the plasma-field interface.

From Equation (229) with $V_{\mathrm{ex}}=2 \cdot 10^{7} \mathrm{~cm} / \mathrm{s}$ (specific impulse is $20000 \mathrm{~s}$ ), the growth rate for $M_{\theta}=5: \gamma_{\mathrm{RT}} \approx 1.6 \cdot 10^{6} \mathrm{~s}^{-1}$. For example, suppose that the longitudinal extent of the region of adverse curvature is only $10 \mathrm{~cm}$, and $V_{/ /} \approx 10^{7} \mathrm{~cm} / \mathrm{s}$ 
within the nozzle. Then the transit time through that adversecurvature region would be about $10^{-6} \mathrm{~s}$. From the product of growth rate and transit time, we then infer that there would occur only one or two e-foldings of the most unstable RT mode. In this particular example, the RT flute instability therefore appears to be harmless.

An expression for the growth rate of the most-unstable mode, as determined by the presence of ion gyroviscosity, can be obtained without the above-mentioned approximations (i.e., replacing density and temperature by their breech values). The resulting expression is valid anywhere along the nozzle. The procedure employed is that the maximum $k$ or $M_{\theta}$ allowed by ion gyroviscosity according to Equation (231) is used in the RT growth rate, Equation (228). As before, the ion gyroradius at a representative point within the plasma-field interface is expressed in terms of the ion inertia length of the neighboring core plasma, by $a_{i}^{2}=\beta\left(c^{2} / \omega_{p i}^{2}\right)$.

Here, $\beta(\approx 1)$ is the local total $\beta$ at a representative point within the plasma-field mixing layer, but $\omega_{p} i$ is the ion plasma frequency evaluated within the core plasma. The average ion number density within the interface has been set to half the core density.

Taking the maximum wave number $k$ from Equation (231) into the growth rate Equation (228) and using the explicit expression for the ion plasma frequency, one then finds that the growth rate of the most unstable mode, as limited by ion gyroviscosity, can be written as follows:

$$
\begin{aligned}
\gamma_{\mathrm{RT}} \approx & {\left[\left.\frac{V_{\mathrm{ex}}}{R_{\mathrm{eq}}^{2 / 3}\left(c / \omega_{p i}\right)_{b}^{1 / 3}}\right|_{\mid}\right.} \\
& x_{\{}^{\}_{j}}\left(\frac{5}{\beta}\right)^{1 / 6}\left(\frac{n}{n_{b}}\right)^{1 / 6}\left(\frac{T_{b}}{T}\right)^{1 / 6}\left[1-\left.\left.\frac{3}{5}\left(\frac{n}{n_{b}}\right)^{2 / 3}\right|_{]} ^{2 / 3}\right|_{\}} ^{\}}\right.
\end{aligned}
$$

Subscript " $b$ " refers to the value of the quantity in the breech or beginning of the nozzle. This result is valid at any longitudinal position along the nozzle, provided that ion gyroviscosity constitutes the dominant modification to the high-conductivity plasma fluid model.

It is clear from Equation (237) that the principal dependence of the RT growth rate, as limited by ion gyroviscosity, occurs just in the first bracketed quantity. That quantity contains the propellant's exit velocity (specific impulse) in the numerator, the breech value of the ion inertia length in the denominator, and the local adverse radius of curvature $\left(R_{\text {eq }}>0\right)$ in the denominator.

$$
\gamma_{\mathrm{RT}} \approx \frac{V_{\mathrm{ex}}}{R_{\mathrm{eq}}^{2 / 3}\left(c / \omega_{p i}\right)_{b}^{1 / 3}}
$$

The $R_{\text {eq }}$ varies along the flow at the self-consistent plasmafield interface. In regions of good curvature, $R_{\text {eq }}$ is negative and there is no instability. The other two factors in Equation (238) have their positions fixed at the exit and breech.

The quantity in braces in Equation (237) is of order unity and is insensitive to temperature and density along the flow field. Using the adiabatic relation between $T$ and $n$ along the flow, the product of the density ratio and the inverse temperature ratio in Equation (237) reduces just to $\left(n / n_{b}\right)^{1 / 18}$, which is essentially just 1.0. In Equation (237), the braced quantity also is very insensitive to the value of local $\beta$ within the plasma-field interface. Even the last internal factor in brackets is always between 0.5 and 1.0 , but it can be explicitly taken into account if desired.

The one quantity in Equation (237) whose variation with longitudinal position along the flow is certainly needed is the self-consistent radius of curvature $R_{\mathrm{eq}}$ of the magnetic field at the edge of the plasma. This quantity can be extracted from axisymmetric MHD simulations of flow through magnetic nozzles. The cylindrical radius $r$ of the propellant is notably absent from the growth rate in Equation (237).

Thus, for design purposes the reduced versionEquation (238) - is probably sufficient. One could try to apply it to design the self-consistent magnetic nozzle field to be such that, in a region of adverse curvature, the product of growth rate and transit time of propellant flow through that region is less than 1. A fallback position is to totally avoid the presence of adverse magnetic curvature. However, achievment of that goal may be hindered by adverse selfconsistent deformation of the original vacuum magnetic field. Such adverse deformation may be difficult to avoid in the injection of hot plasma into a premagnetized nozzle tube that is burdened by constrained dimensions or by the discreteness and separation of magnetic source coils.

Equation (238) constitutes a simple practical formula for the RT growth rate at any longitudinal position in the magnetic nozzle. It should nevertheless be regarded as provisional. For example, one might be concerned that downstream of the nozzle throat the propellant temperature eventually decays to the point that ion-ion collisions become significant, implying that ion gyroviscosity should be replaced by ion collisional viscosity. However, almost the opposite proves to be the case. The ion-ion collision frequency $v_{i}$ changes as $n / T^{3 / 2}$, so is constant along the propellant's flow, since adiabatically, $T \sim n^{2 / 3}$. The ion-ion mean free path, however, varies as $\lambda_{i} \sim V_{\text {th } i} / v_{i} \sim n^{1 / 3}$. Thus, the 
kinematic viscosity varies as $\lambda_{i} V_{\text {th } i} \sim n^{2 / 3}$, which decreases towards the exit plane. In contrast, taking magnetic pressure balance into account, the gyroviscous diffusivity $a_{i} V_{\text {th }}$ varies as $n^{-1 / 6}$, slightly increasing towards the exit plane. Thus, gyroviscosity remains the dominant viscous effect as long as $\omega_{c i}>v_{i}$. While the condition $\omega_{c i}>v_{i}$ remains in force within the plasma-field interface, flute-mode dynamics in the plane perpendicular to $\mathbf{B}$ are governed by ion gyroviscosity rather than collisional viscosity. Furthermore, for relevant injected-plasma parameters, the collisional situation $\omega_{c i}<v_{i}$ would require plasma density to decrease substantially from its injected value, by at least an order of magnitude. The $v_{i}$ is practically constant along the adiabatic flow, whereas $\omega_{c i}$ decreases as $n^{5 / 6}$. But, at that point, the propellant has practically reached the nozzle exit anyway. In a more detailed model, there would also be an additional intermediate viscous diffusivity (Ref. 9) of the form $D_{\text {vis } i} v_{i} / \omega_{c i}$, but this amount of detail lies beyond the present purview.

On the other hand, the Hall effect has been neglected in this modeling of the RT instability. Huba (Ref. 33) has pursued the subject of Hall MHD, and has found that at shorter wavelengths $\sim \mathrm{c} / \omega_{p} i$, the Hall effect, taken alone, increases the growth rates of those flute modes, and changes their nonlinear structure as well. Actually, there is a competition between enhancement of growth by the Hall effect and mode stabilization by ion gyroviscosity, a subject addressed later by Huba and Winske (Ref. 38). The Hall effect should be dominant in cold plasmas, such as in the beginning phase of theta-pinch implosions exemplified by Reference 34 and in the expansion of laser-produced plasmas, Reference 17. Ripin et al. experimentally confirmed the features of the HallMHD model (Ref. 17). MHD modeling of flow through magnetic nozzles should be revisited in this regard, so that Hall effects, together with ion gyroviscosity, can be applied in a knowledgeable manner to "adverse curvature" instabilities in propellant flow through magnetic nozzles.

That Hall effects can occur in a cold plasma is correct and can be understood by considering that the generalized Ohm's law (mks units) does not explicitly contain thermal effects; yet, it contains the Hall term (as the second term on the right-hand side) as a contribution to the Hall electric field (see Eq. (37b)). Nevertheless, in specific applications to plasma-nozzle flow, thermal effects can sometimes creep in when estimating the importance of the Hall term.

$$
\mathbf{E}+(\mathbf{V} \times \mathbf{B})=\eta \mathbf{J}+\frac{\mathbf{J} \times \mathbf{B}}{n q}
$$

The first term on the right in Ohm's law is the resistive term. Here, $\eta$ is the resistivity. Equation (239) is just the electron-fluid momentum equation without the electron inertia and without the electron pressure gradient. The absence of the latter emphasizes the circumstance that here we are considering "cold plasma."

A necessary condition for the Hall term to be important is that it not be dominated by the resistive term. This requirement is easily shown to be equivalent to having the electron gyrofrequency be large compared to the electron collision frequency with the ions (or with plasma fluctuations in the case of microturbulence). Such a large gyrofrequency and relatively small collision frequency is to be expected in the plasma-field interface of the magnetic nozzle for the parameters of interest. The size of the system in the RT problem is represented by the azimuthal wavelength of modes.

If the above requirement is satisfied (small enough electron collision frequency), then the next step in estimating the importance of the Hall term is to compare it with the motional electric field term, $\mathbf{V} \times \mathbf{B}$. Here, $\mathbf{V}$ is the local fluid velocity of the plasma, which is the same as the ion-fluid velocity. Also, B is the local magnetic field vector. This particular comparison will be made in the following example of the coaxial MHD thruster, where it will be shown that the Hall effect is important when the ion gyroradius is not too small compared with the size of the system.

\subsubsection{Example: Estimate of Hall Term in Coaxial MHD Thruster}

The salient feature of the following example is that the Hall effect becomes important when the ion gyroradius becomes comparable to the macroscopic size of the system. In the RT problem, the size of the system is represented by the azimuthal wavelength of modes.

In the coaxial plasma thruster, there are inner and outer coaxial electrodes, which form an annular nozzle; plasma flow velocity $\mathbf{V}$ is in the longitudinal direction, magnetic field $\mathbf{B}$ is azimuthal, and current density $\mathbf{J}$ is radial between the electrodes. Hence the strength of $B$ decreases in the longitudinal direction, as required by Ampere's law $\nabla \times \mathbf{B}=\mu_{0} \mathbf{J}$.

From Equation (239) we want to compare the magnitudes (mks units) of the motional electric field term $V B$ and the Hall term $J B / n q$ :

\section{$V B \quad$ versus $J B / n q$}

Suppose that $V$ is about the same as the ion thermal velocity, $V_{\text {th } i}$, which, at any rate, is a desired feature of nozzle flow. When the electric field term is comparable to the Hall term, then the Hall effect becomes significant.

The following relationships are used:

$\omega_{p i}^{2}=n q^{2} / \varepsilon_{0} m_{i}$, where $\omega_{p i}$ is the ion plasma frequency

$\varepsilon_{0} \mu_{0}=1 / c^{2}$, where $c$ is the speed of light 
$V_{A} / \omega_{c i}=c / \omega_{p i}$, where $V_{A}$ is the Alfven speed and $\omega_{c i}$ is the ion gyrofrequency

$$
V_{\text {th } i} / V_{A}=\sqrt{\beta_{i}}
$$

Then the above comparison can be reduced as follows:

$$
\begin{array}{ccc}
V B & \text { versus } & J B / n q \\
\downarrow & & \downarrow \\
\sqrt{\beta_{i}} & \text { versus } & \left(c / \omega_{p i}\right) / L_{\text {coax }}
\end{array}
$$

Here, the quantity $\beta_{i}$ signifies the local ratio of ion pressure to magnetic pressure, and $L_{\text {coax }}$ is the coaxial longitudinal distance over which the azimuthal magnetic field becomes depleted by pushing the plasma downstream. Thus $L_{\text {coax }}$ is the length of the annular nozzle.

The thermal ion gyroradius $a_{i}$ is related to the ion inertia length $c / \omega_{p i}$ by $a_{i}=\sqrt{\beta_{i}}\left(c / \omega_{p i}\right)$, so the above comparison finally reads

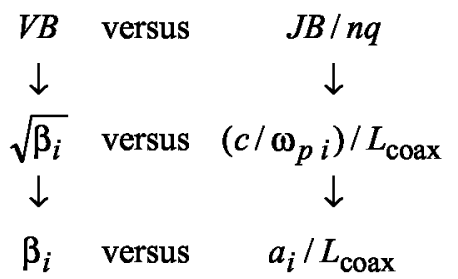

In realistic situations in the coaxial thruster, $\beta_{i}$ can be of order 1. As the ions flow downstream they make gyrations in the r,Z-plane, around the azimuthal magnetic field lines. Thus, if the ion gyroradius $a_{i}$ is not too small compared with $L_{\text {coax, }}$ so that the ions undergo only a few magnetogyrations as they flow through the coaxial nozzle, the term on the right can be important, and concomitantly, the Hall effect is important. In the case of flute modes, the azimuthal wavelength is the "size of the system."

\subsubsection{Concluding Thoughts}

In the flute-mode discussion of the RT instability of this report (in Sec. 4.2.2, "Surface Instability of Flowing Propellant in a Region of Adverse Curvature"), a similar effect is noted in the literature, since the ion skin depth $\left(c / \omega_{p}\right)$ is nearly the same as the thermal ion gyroradius when $\beta$ is near 1 . A paper by Huba and Winske (Ref. 38) contains a detailed account of the RT instability when both Hall effects and ion gyroviscosity (FLR or FLR stabilization) are active. Linear stability analyses and nonlinear numerical simulations based on both fluid and kinetic models were carried out in that paper. It is instructive to consider why this pioneering paper is not yet applicable to the magnetic nozzle configuration.
First, in Reference 38, the disparate light and heavy fluids, which constitute the RT configuration, are separated by a planar interface in the presence of an artificial gravitational force. Thus, geometric effects are missing. There is the effect of lateral pressure forcing a wedge-shaped element radially outwards (see Fig. 5). A concomitant effect is the outward spatial decay of external field strength in the region of adverse curvature. These geometric effects of adverse curvature are essential for the derivation that produces the pressure contribution to the growth rate in Equation (226). They are especially required since artificial gravity is not invoked in our model.

Second, the models used in Reference 38 are "low $\beta$ " models. This assumption has two consequences. The magnetic field changes only slightly in traversing the interface, in contrast to the magnetic field profile in the interface of the magnetic nozzle. Moreover, the ion gyroradius $a_{i}$, important for FLR stabilization, is small against the ion inertia length, $c / \omega_{p}$, in Reference 38. The latter is important for Hall destabilization. In contrast, both of these lengths are comparable in the magnetic nozzle interface, because $\beta$ is of order 1 there. Thus, short azimuthal wavelengths would be simultaneously influenced to roughly equal extents by both processes in the magnetic nozzle. In Reference 38, however, the Hall effect was dominant because the ion inertia length was much larger than the ion gyroradius.

Since Equation (238) is based only upon FLR stabilization, one may question whether it provides meaningful engineering guidance. It does so by eliminating from consideration those plasma profiles that are RT unstable. That is, if a configuration were found to be RT unstable in spite of including FLR stabilization effects in the model, then surely it would prove to be yet more unstable had Hall destabilization effects also been included. On the other hand, if a stable configuration is found in the sense that $\gamma_{\mathrm{RT}} t<1$, there is then no certainty that such a configuration would remain benign if Hall effects had been present. Thus, further work is needed in this regard.

Thus, in the flute-mode RT instability, it has been found by Huba and Winske (Ref. 38) that the Hall effect becomes important and destabilizing when the macroscopic wavelength is on the order of the ion gyroradius, but also it is known that the FLR stabilization effect becomes important under the same conditions. Thus the two effects work against one another. This process constitutes a delicate balance that deserves a careful calculation oriented to the magnetic nozzle. The paper of Huba and Winske shows that the two effects are in opposing directions, but those special calculations are artificial and constructed to clearly show both effects by turning one on after the other is on. That paper is not directly applicable to the magnetic nozzle, however; a special calculation focussed on the magnetic nozzle problem is therefore needed.

In conclusion, it is indeed the case that the model of the flute-mode RT instability in this report, which includes FLR 
stabilization but omits the Hall effect, may be giving incorrect results by not including both effects at the same time. That is to say, a traditional approach was followed, consisting of subjecting the ideal MHD results to FLR stabilization, without regard to any additional effects. That is exactly why it is suggested that there exists a need for the more complete calculation in Section 4.2.2, since if the RT instability appears threatening even when including FLR stabilization effects, it would appear even worse if the Hall destabilization effect were also included. In this sense, the formula Equation (238) is a meaningful engineering design criterion.

\subsection{Short-Wavelength Gravity-Driven Instabilities}

In this section, we shall examine ion gyroviscous stabilization of the unstable MHD flute modes associated with adverse longitudinal curvature. It is also pointed out that the destabilizing Hall effect should ultimately be included selfconsistently in the model so as to obtain a more complete picture of the behavior of these modes.

Up to this point, discussion of RT-type instabilities has been carried out within the fluid description and has been directed to flute modes of long wavelengths compared with the thickness of the plasma-field interface. The manifestation of these long wavelength modes is the azimuthal deformation of the interface as an entity.

However, it was recognized by Davidson and Gladd in Reference 18, and by Gary and Thomsen in Reference 35, that ion inertial force, or effective gravity, can drive instabilities of the LHD type within the interface layer itself. These unstable flute modes have very short wavelengths, which range between thermal ion gyroradius $a_{i}$ and thermal electron gyroradius $a_{e}$. They are not stabilized by finite iongyroradius effects, except at very small current drift velocities where the unstable wavelengths become as large as the ion gyroradius (see Ref. 19).

The salient observation regarding the short-wavelength LHD flute modes, as connected to the presence of effective gravitational acceleration, is that the treatment given in Section 3.0 of this report, "Resistivity From Gradient-Driven Microinstabilities," applies without modification (see Ref. 18). The reason is that the treatment is set in terms of the current-drift velocity $V_{d}$. The reader will recall that the ratio $V_{d} / V_{\text {th } i}$ is the characteristic driving factor for the real part of the frequency of this drift mode, and its square is the characteristic driving factor for the growth rate of the instability (see Eq. (144)). Moreover, the square of this velocity ratio also is a principal feature of the anomalous resistivity arising from LHD microturbulence, as was discussed in Section 3.0. Thus, the theory in terms of $V_{d} / V_{\text {th }} i$ remains intact.

The role of gravity in the LHD modes only becomes apparent when the expression for the current drift-velocity itself is examined. This expression will now be developed within the context of propellant flow in the magnetic nozzle.
For simplicity of discussion electron temperature will be neglected, but this is not an essential restriction. The ion macroscopic momentum equation is written in a frame of reference that is instantaneously at rest in the ion fluid; nevertheless, ion inertial effects (ion-fluid acceleration) can appear in this frame.

$$
\partial_{x} P_{i}-\rho g_{x \text { eff }}=n q E_{x}
$$

The $\mathrm{x}$-direction is the quasi-radial direction, outwards across the external magnetic flux. The effective gravitational field is $g_{x \text { eff }}=V_{/ /}^{2} / R_{\mathrm{eq}}$, with $V_{/ /}$the propellant flow velocity along $\mathbf{B}$ and $R_{\mathrm{eq}}$ the local equilibrium radius of adverse curvature. The magnetic field vector defines the local z-direction. The electron drift velocity $V_{d} y_{e}=$ $\left[(\mathbf{E} \times \mathbf{B}) / B^{2}\right]_{\mathrm{y}}$ in the y- (azimuthal) direction is obtained by dividing Equation (240) by $n q B$. The result is (in mks units)

$$
V_{y d e}=\frac{T}{q B} \frac{1}{x_{n}}+\frac{V_{l /}^{2}}{\omega_{c i} R_{\mathrm{eq}}}
$$

Here, we have assumed uniform ion temperature and have defined the density gradient length within the interface by $\left(1 / x_{n}\right)=h^{-1} \partial_{x} n \mid$. Earlier in this report, the interface width was described as $\delta$, so $x_{n}=\delta$.

Now, throughout most of the nozzle, the flow velocity is somewhat comparable to the local sound speed, which, in turn, is comparable to the ion thermal velocity. Therefore, Equation (241) says that, in general, the gravitational term is not important unless $R_{\text {eq }}$ is small enough to be comparable to $x_{n}$. Such is clearly not the case throughout most of the nozzle: $R_{\text {eq }}$ is likely several tens of centimeters, whereas $x_{n}$ is on the order of just a few centimeters. We conclude that gravitational acceleration due to adverse curvature generally should constitute only a minor modification to the edgelayer gradient that drives the short-wavelength LHD flute instabilities.

The exception to this conclusion occurs at the point of impact of injected plasma with magnetic flux. It was seen earlier that even at near-grazing incidence of injected flow streamlines with the ambient magnetic field, the local radius of curvature at the point of impact would be so small as to lie beyond the purview of a fluid model. Its scale of smallness would probably be set by "microscopic" lengths, such as $a_{i}$, or by $c / \omega_{p i}$. Only then would the two contributions to $V_{d}$ become comparable in Equation (241). Thus, it is indicated that the initial point of impact of plasma with field in the injection region be scrutinized with respect to modification of LHD flute modes by gravitational effects.

Near the exit plane, the second (gravitational) term in Equation (241) would increase (as $n^{-5 / 6}$ ) faster than the first 
term (as $n^{-1 / 6}$ ), unless the adverse radius of curvature $R_{\text {eq }}$ is maintained at a large value by judicious arrangement of downstream magnetic coils.

\subsection{Summary and Conclusions}

The principal objective of this report was to ascertain the degree of attachment of propellant plasma to magnetic nozzle flux, as represented by the spatially evolving width of the plasma-field mixing layer, and to explore ways of mitigating that attachment. However, it was emphasized at the outset that even attached plasma undergoes converging-diverging nozzle acceleration along with core plasma. Moreover, reference was made to extant calculations showing that attached plasma can be resistively detached downstream with little loss of efficiency. This is provided that the necessary weak divergence of the nozzle in a long detachment region supported by trim coils is compatible with the overall mission design of the vehicle.

The four principal results from this report are as follows:

(1) The initial thickness $\delta$ of the plasma-magnetic field interface in the nozzle breech $b$ (where the hot plasma is injected) is derived. It is $\delta_{b} \approx 2 c / \omega_{p}$, which serves as the initial condition for resistive broadening of the interface along the flow. Here, $c$ is the speed of light in vacuum and $\omega_{p i}$, the ion plasma frequency in the breech. For the parameters of interest here, $\delta_{b}$ is a few centimeters.

(2) The subject of gradient-driven Lower Hybrid Drift (LHD) microturbulent (anomalous) resistivity $\eta_{a}$ is reviewed and compared with the classical resistivity $\eta_{\mathrm{cl}}$ that arises from electron coulomb scattering on the ions. The results from the smaller of two predictions for $\eta_{a}$ are summarized. This subject bears on the spatial rate of resistive broadening of the interface, for which a simple algorithm is derived in terms of a general resistivity. The parameter dependencies of $\eta_{a}$ appear to be agreed upon in various models, but the large discrepancies in the numerical coefficient need to be resolved.

(3) The subject of flute instabilities of the magnetically confined interface in regions of adverse magnetic curvature is addressed. A physical derivation of the growth rate is performed for curved geometry without artificial gravity, which includes the effects of both pressure and flow. A practical formula for the growth rate is obtained that takes into account flute-mode stabilization by ion gyroviscosity (finite Larmor radius (FLR) stabilization). The formula can be used in magnetic nozzle design, by monitoring the self-consistent plasma-nozzle shape and flow velocity in axisymmetric simulations of propellant flow through magnetic nozzles. In this manner, one can estimate the extent of e-folding of flute instabilities in regions of adverse curvature.
(4) The relevance of the Hall effect to Ohm's law is clarified. The simple Ohm's law used to connect azimuthal current density with azimuthal motional electric field in calculating resistive interface broadening depends on the Hall voltage not being shorted out by a flow of Hall current. This subject is important because the electron gyrofrequency $\omega_{c e}$ far exceeds the electron collision frequency $v_{e}$ within the plasma-magnetic field interface. A complementary example is provided whereby the full Hall current is allowed to flow; and concomitantly the plasma is allowed to rotate. A critical nozzle length is identified below which the interface thickness is limited to about one ion gyroradius $a_{i}$. The critical length is $\lambda_{e} \sqrt{m_{i} / m_{e}}$, where $\lambda_{e}$ is a representative electron mean free path and $m_{i}$ and $m_{e}$ are the ion and electron masses, respectively.

In this study, the basic features of converging-diverging nozzle flow were reviewed. An algorithm was identified to represent resistive broadening along the flow, of the plasmafield mixing layer. The results for the evolving width of the mixing layer proved to be essentially the same for two distinct models: diffusion of plasma into magnetic field and diffusion of magnetic field into plasma. Assuming a zerowidth starting condition for the mixing layer, so as to compare with some reported resistive magnetohydrodynamics (MHD) simulations, the simulations were found to be rather more effectively diffusive than would be predicted by classical resistivity.

Resistive MHD simulations sometimes take for granted the simple form of Ohm's law, despite the presence of a strong longitudinal magnetic field in the mixing layer. Thus, the magnetic field produces the condition, $\omega_{c e} \gg v_{e}$. Invoking the simple form of a local Ohm's law that incorporates unmagnetized electrical conductivity, to relate azimuthal current density to azimuthal motional electric field, actually requires that the Hall voltage is not shorted out. In other words, it is required that no Hall current be allowed to flow. Therefore, global electrophysical boundary conditions in the device ought to be kept in mind when assessing the validity of this local Ohm's law. In order to suppress the Hall current, resistive MHD simulations (and experiments) should have an insulating wall layer inserted between the metallic field coils and the plasma. Such a wall layer also aids in protecting the field coils. (However, plasma near the wall might longitudinally short out the insulating wall layer and thereby enhance a closure path for the Hall current.)

The opposite condition, zero Hall voltage, therefore was also explored in this report. If the Hall current were indeed allowed to flow, as well as to self-consistently spinup the plasma propellant, then it might be possible to limit plasma attachment to magnetic nozzle flux. Specifically, we found that if the nozzle's length were held smaller than a critical length $\lambda_{e} \sqrt{m_{i} / m_{e}}$, then the width of the plasma-field interface layer could be held to about one ion gyroradius (here, 
the initial interface-layer thickness was neglected, except in one previously discussed exception). If Hall current could be maximized in a magnetic nozzle of subcritical length, then the detachment problem at the nozzle's exit would be reduced to a secondary issue. It was also pointed out that the associated rotational spinup energy would be recovered in the diverging portion of the flow field.

It is necessary to specify a spatial initial condition in the injection region, for calculating the downstream evolution of the width of the plasma-field mixing layer. Moreover, its width characterizes the anomalous resistivity that influences that very evolution. Therefore, special attention was given to the mixing-layer's width $\delta=x_{n}$ in the injection (breech) region of the magnetic nozzle. From first-principles physics considerations, it was inferred that it would be somewhat more than twice the ion inertia length, $c / \omega_{p} i$ (in terms of the internal ion number density). Concomitantly, it was estimated that the ion gyroradius $a_{i}$ would be only about one-half to onethird of $\delta$. In addition, it was shown that the ratio $a_{i} / \delta$ is insensitive to position between breech and throat of the nozzle.

This result for $a_{i} / \delta$ determines the ratio of electron current drift-velocity to ion thermal velocity. That velocity ratio, in turn, is a principal factor in the growth rate of LHD modes as well as their subsequent evolution into microturbulent resistivity.

The LHD instability was singled out for explaining the evolution of edge gradients of magnetically confined plasmas, because it is an instability that is easier to excite than other possible microinstabilities. For example, in comparison to the ion-acoustic instability, the current drift-velocity need not be large, and the electron-ion temperature ratio also need not be large. The ion-acoustic instability and an associated empirical resistivity had been previously invoked to explain the structure of magnetically imploded theta-pinches. Moreover, because the LHD mode is basically a non-cyclotronresonant mode in regards to the ions, it is robust enough to saturate at a significant level of fluctuations. This behavior presents a contrast with the well-studied electron-cyclotron drift instability, which has a very high growth rate but is basically a cyclotron-resonant electron mode easily destroyed by nonlinearity.

The derivation of the small-amplitude linear LHD mode was outlined and its properties at maximal growth rate were summarized. The properties show a drift-wave behavior, with a wave phase velocity $V_{\mathrm{ph}}=\operatorname{Real}(\omega) / k$, proportional to current-drift velocity $V_{d}$. The characteristic wave number $k$ is on the order of the reciprocal electron gyroradius $a_{e}$. The wave's temporal growth rate is a fraction of the lower hybrid gyrofrequency $\sqrt{\omega_{c e} \omega_{c i}}$. Moreover, the growth rate is proportional to $\left(V_{d} / V_{\text {th } i}\right)^{2}$. In the ion-cyclotron drift regime, with magnetized ions and very small $V_{d}$, the growth rate is so small as to have little relevance to the transit time in the magnetic nozzle application.
The linear characteristics of the LHD wave were then invoked in a derivation of the quasi-linear theory of wave saturation. The saturated level of electric field fluctuations is a principal factor in the size of the anomalous resistivity produced by the wave. Use of the wave-energy bound, in which the total wave-fluctuation energy (not just electric-field-fluctuation energy) is obtained from electron drift inetic energy, produced a much smaller resistivity than did the Fowler bound. The resistivity from the wave-energy bound has parameter dependence identical to some alternative models of wave saturation and is notably similar to a first-principles electromagnetic particle simulation (Ref. 14) by Brackbill et al. The latter, however, produced a numerical factor in the resistivity that is about one order of magnitude smaller than in the quasi-linear model with the wave-energy bound. The explanation may lie in wave-wave coupling to damped modes in the simulation, except that Drake et al. (Ref. 26) also consider wave-wave coupling and yet find a much larger numerical coefficient.

One principal observation of this report is that several alternative models of the anomalous resistivity in the interface layer do agree on the multiparameter dependencies of $\eta_{\mathrm{a}}$. The second principal observation is that there are large discrepancies in the numerical value of the coefficient $\eta_{a}$ predicted by these models. For example, the model of Drake et al. (Ref. 26) has a numerical coefficient almost 2 orders of magnitude larger than the largest value from the simulations of Brackbill et al. (Ref. 14). This disagreement in the numerical coefficient needs to be resolved in order for the subject of anomalous resistivity to be applied with confidence to engineering design of the magnetic nozzle.

The anomalous resistivity $\eta_{a}$ extracted from the simulation runs of Brackbill et al. was expressed in terms relevant to the plasma-field interface in the magnetic nozzle concept, facilitating a comparison to classical resistivity $\eta_{\mathrm{cl}}$ arising from coulomb collisions of electrons with ions. The results are displayed in Figure 4. For example, at hydrogen-ion densities near $10^{15} \mathrm{~cm}^{-3}, \eta_{a}$ is dominant over $\eta_{\mathrm{cl}}$ at injectedplasma temperatures exceeding about $200 \mathrm{eV}$. However, if the results of Drake et al. are correct, then a breech temperature exceeding only $20 \mathrm{eV}$ would be sufficient for anomalous resistivity to become dominant.

Also, an open question remains as to the correctness of the $\beta$ dependence of $\eta_{a}$, which purportedly was extracted from the Brackbill et al. simulations by comparison with the plasma heating results of Gary (Ref. 24). This is a very important question because the local $\beta$ of the magnetically confined plasma varies from zero outside the plasma to $\gg 1$ inside the plasma, in traversing the edge-plasma gradient. Thus, resolution of this question affects the ability of resistive MHD simulations to properly represent the internal evolution of the plasma's edge gradient, hence to represent the evolution of the width of the plasma-field interface. The difficulty with the $\beta$ dependence proposed in Reference 14 is 
outside the scope of this report. We note the contrasting result of Drake et al., in which no $\beta$ dependence is claimed even though their simulations employ an electromagnetic model.

We conclude this summary with a few remarks on the Rayleigh-Taylor- (RT-) type instability. The presence of RT-type flute modes in regions of adverse curvature represents a loss of axisymmetric nozzle-based control of the flow of propellant. This loss of control can be mitigated by having the growth of the mode with the maximum mode number be limited by the available transit time of propellant through the adverse region.

A physical derivation was given of the growth rate of RT-type flute modes downstream from the injection region in magnetically confined plasma in regions of adverse magnetic curvature. As is appropriate to the magnetic nozzle, the derivation incorporated the joint effects of propellant pressure and propellant flow (see Eq. (226)). A practical formula was obtained for the growth rate (Eq. (238)), which takes into account the stabilization of RT-type flutes of the higher mode numbers by the effects of collisionless ion gyroviscosity FLR stabilization).

Within the injection region, the point of initial oblique impact of propellant with external nozzle magnetic field was singled out as having a localized region of very severe adverse curvature in the flow streamline. The extreme curvature there is due to the strong eddy current braking associated with the crossfield motion of injected hot plasma of high electrical conductivity. It was recognized that the structure of this region and the instabilities therein are inaccessible to a fluid model. It was suggested that the region of initial meeting of plasma with excluded flux receive special consideration regarding gravitational effects on shortwavelength flute modes that are closely related to the LHD instability.

Lastly, there are effects of hot-plasma physics not covered in the fluid-based modeling of Huba on the FLR and Hall effects. These hot plasma kinetic effects should be taken into account with regard to modes driven by adverse curvature, even in modes that are not limited to short wavelengths, and are, therefore, also additional to the short wavelength effects existing in the LHD-type modes. Here, we exclude the ballooning modes that were already mentioned with regard to Reference 36.

We were careful to base our physical derivation of elongated flute instabilities on the presence of adverse magnetic curvature rather than on artificial gravity. It is nevertheless expeditious for purposes of incorporating more plasma physics to relinquish the curved geometry in favor of a plane interface with uniform artificial gravity. With this artifice, Migliuolo (Ref. 39) generalized the famous FLR-flute mode theory of Rosenbluth et al. (first reference in Ref. 39) to extend to more plasma-physical effects. In particular Migliuolo included electromagnetic effects, nonzero $\beta$ effects, and the effects of particle drift-resonances azimuthally across the magnetic field. Thus, Reference 39 shows how to obtain more accurate and more comprehensive properties of gravity-driven flute modes. He also explains that the short wavelength modes treated in Reference 35 do not contain electromagnetic effects.

Also, Freidberg and Wesson (Ref. 40) used the same artifice to show that conditions could arise wherein flute modes are essentially stabilized by FLR, but at the same time, gravitydriven oblique modes are unstable. The latter are driven by resonant ion motion along the magnetic field, which is sometimes able to supply the extra energy needed to bend magnetic field lines. In magnetic nozzles containing regions of self-consistent adverse curvature traversed by hot plasma, it therefore would be important to apply appropriately detailed plasma-physical models to accurately describe and deal with the resulting instabilities.

It is possible to include more detailed plasma-physical models in the analysis of surface perturbations of curved plasma boundaries by neglecting the surface curvature and instead introducing an artificial gravity. The relevance, however, of these artificial models to the integrity of the magnetic nozzle has not yet been demonstrated.

In 2005, Arefiev and Breizman published a paper (Arefiev, A.V.; and Breizman, B.N.: Magnetohydrodynamic Scenario of Plasma Detachment in a Magnetic Nozzle. Phys. Plasmas, vol. 12, 2005.) in which they show that in a highly conductive plasma - where the flow kinetic energy density exceeds the ambient magnetic energy density - the plasma can stretch the magnetic field lines to infinity and thereby escape. (A follow-on paper published in 2008 (Breizman, B.N.; Tushentsov, M.R.; and Arefiev, A.V.: Magnetic Nozzle and Plasma Detachment Model for a Steady-State Flow. Phys. Plasmas, vol. 15, 2008.) presents a model that includes kinetic ions, but not electron-ion collisions, so resistivity was still absent.) However, here in the present report, it is found that the temperature of the plasma drops downstream of the throat of the nozzle. This is also shown by George Marklin's numerical calculation in Reference 6 (Gerwin, Richard A., et al: Characterization of Plasma Flow Through Magnetic Nozzles. AL-TR-89-092 (LA-UR-89-4212), 1989.). Hence, resistivity becomes important, precluding the stretching of field lines. Thus we conclude that the detachment of plasma from magnetic field lines remains an important process.

In summary, there are many fundamental aspects left to be studied regarding the physics of high-temperature, highnumber-density plasma acceleration by means of the magnetic nozzle. The derivations in this report on selected topics, building on the work of others, can guide further theory development and experimental research in this emerging area of study. Only then can fast, piloted interplanetary travel utilizing magnetic nozzles begin to become viable.

\author{
Glenn Research Center \\ National Aeronautics and Space Administration \\ Cleveland, Ohio, December 11, 2009
}





\section{Appendix A.-Symbols and Expressions}

\begin{tabular}{|c|c|c|c|}
\hline$A$ & plasma cross-sectional area & $I$ & electric current \\
\hline $\mathbf{a}$ & local particle acceleration & $\mathbf{J}$ & current density \\
\hline$a$ & thermal gyroradius & $\{\delta \mathbf{J}\}$ & current density fluctuation \\
\hline B & magnetic field & $J$ & magnitude of current density $\mathbf{J}$ \\
\hline$\{\delta B\}$ & fluctuating magnetic field & $\mathbf{K}$ & azimuthal surface current density \\
\hline$\hat{B}$ & magnitude of magnetic field $B$ & $K$ & $\begin{array}{l}\text { magnitude of azimuthal surface current density } \\
\mathbf{K}\end{array}$ \\
\hline b & unit vector along B & $k$ & azimuthal wave number \\
\hline$C$ & constant & $\mathbf{k}$ & azimuthal wave vector \\
\hline$c$ & speed of light in vacuum & $L$ & length \\
\hline$\{\delta \mathbf{D}\}$ & electric displacement & $\mathbf{l}$ & vector distance along magnetic field line (or \\
\hline$D$ & $\begin{array}{l}\text { diffusion coefficient, arising from resistive } \\
\text { diffusivity }\end{array}$ & $l$ & $\begin{array}{l}\text { streamline in field-free region of core plasma) } \\
\text { magnitude of distance } 1\end{array}$ \\
\hline$D_{y}^{(1)}(k, \omega)$ & Fourier-mode amplitude of $\{\delta \mathbf{D}\}$ & $M$ & mode number \\
\hline $\mathbf{E}$ & electric field (or generalized electric field) & $m$ & mass \\
\hline$\{\delta \mathbf{E}\}$ & electric field fluctuation & $\dot{m}$ & mass flow rate \\
\hline$E$ & magnitude of electric field $\mathbf{E}$ & $n$ & number density \\
\hline$E_{y}^{(1)}(k, \omega)$ & $\begin{array}{l}\text { Fourier-mode amplitude of azimuthal compo- } \\
\text { nent of electric field } \mathbf{E}\end{array}$ & $n_{e}^{(1)}(k, \omega)$ & $\begin{array}{l}\text { Fourier-mode amplitude of electron density } \\
\text { fluctuation }\end{array}$ \\
\hline$e$ & base of natural logarithm, (2.7183) & $\{\delta n\}$ & number density fluctuation \\
\hline$F$ & force density & $P$ & pressure \\
\hline $\mathrm{f}(u)$ & $\begin{array}{l}\text { generic function of a similarity variable in } \\
\text { Appendix C }\end{array}$ & $\begin{array}{l}\{\delta P\} \\
Q\end{array}$ & $\begin{array}{l}\text { pressure perturbation } \\
\text { degrees of freedom }\end{array}$ \\
\hline$f$ & function & $q$ & fundamental electric charge $\left(1.6 \times 10^{-19} \mathrm{C}\right)$ \\
\hline $\begin{array}{l}\{\delta \mathrm{f}\} \\
f_{i}^{(0)}(k, \omega)\end{array}$ & $\begin{array}{l}\text { perturbed distribution function } \\
\text { ion phase-space distribution function in the }\end{array}$ & $R$ & $\begin{array}{l}\text { local longitudinal radius of curvature of field } \\
\text { line }\end{array}$ \\
\hline & equilibrium situation & $\operatorname{Re}$ & Reynolds number \\
\hline$f_{i}^{(1)}(k, \omega)$ & $\begin{array}{l}\text { Fourier-mode amplitude corresponding to ion's } \\
\{\delta f\}\end{array}$ & $\begin{array}{l}\mathbf{r} \\
\hat{\mathbf{r}}\end{array}$ & $\begin{array}{l}\text { particle position } \\
\text { unit vector in radial r-direction }\end{array}$ \\
\hline$G(x)$ & $\begin{array}{l}\text { resistively diffused magnetic field's } \\
x \text {-dependence within plasma }\end{array}$ & $r$ & $\begin{array}{l}\text { radial coordinate (positive toward wall); origin } \\
\text { at throat on nozzle centerline }\end{array}$ \\
\hline$g$ & gravitational field & $S$ & speed of sound \\
\hline$g(u)$ & $\begin{array}{l}\text { first derivative of } f(u) \text { with respect to } u \text { in } \\
\text { Appendix C }\end{array}$ & $\begin{array}{l}T \\
t\end{array}$ & $\begin{array}{l}\text { temperature } \\
\text { time }\end{array}$ \\
\hline $\mathbf{H}$ & 6-dimensional phase space $[\mathbf{r}, \mathbf{v}]$ & $u$ & similarity variable \\
\hline$h$ & $\begin{array}{l}\text { fraction of radial fluid velocity lost by decele- } \\
\text { ration }(h \leq 1)\end{array}$ & $\mathbf{v}$ & macroscopic (fluid) velocity \\
\hline
\end{tabular}




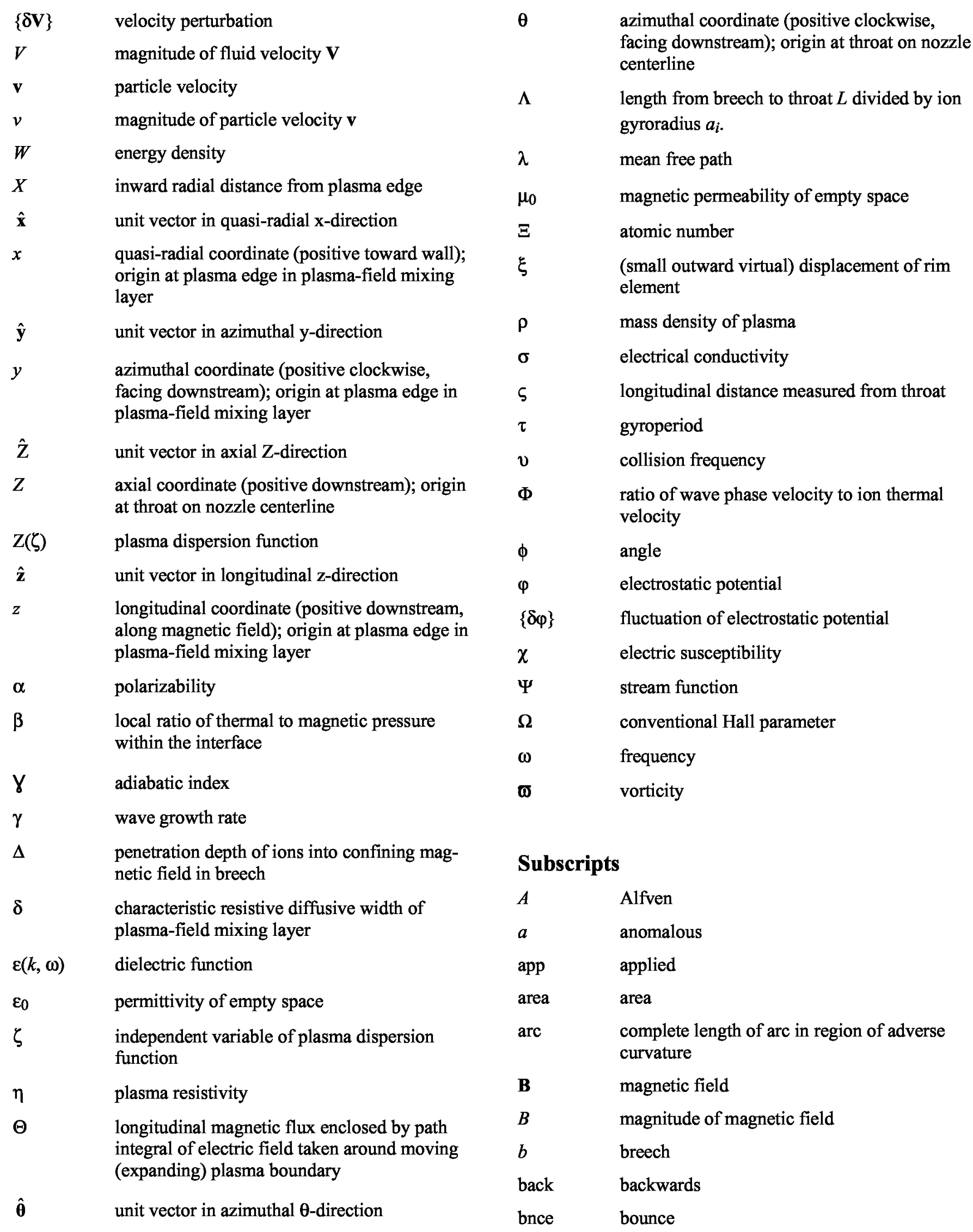




\begin{tabular}{|c|c|c|c|}
\hline Brack & Brackbill & $\mathrm{ph}$ & wave phase \\
\hline$c$ & cyclotron & $R$ & longitudinal radius of curvature \\
\hline char & characteristic & $r$ & radial vector component \\
\hline $\mathrm{cl}$ & classical & ran & random \\
\hline $\operatorname{coax}$ & coaxial & RT & Rayleigh-Taylor \\
\hline core & core & rot & rotating \\
\hline crit & critical & $s$ & species \\
\hline cur & (adverse) curvature & $t$ & throat \\
\hline$D$ & diffusion & tot & total \\
\hline$d$ & drift & th & thermal \\
\hline Drake & Drake & $\operatorname{trans}$ & transient \\
\hline$e$ & electron & $\mathbf{V}$ & velocity \\
\hline eff & effective & vis & viscous \\
\hline eq & equilibrium & wave & wave \\
\hline es & electron sound & $x$ & quasi-radial coordinate (positive toward wall); \\
\hline ex & exit & & layer \\
\hline ext & external & $y$ & azimuthal coordinate (positive clockwise, \\
\hline $\mathrm{fl}$ & fluctuating & & $\begin{array}{l}\text { facing downstream); origin at plasma edge in } \\
\text { plasma-field mixing layer }\end{array}$ \\
\hline$G$ & global & & \\
\hline$g$ & $\begin{array}{l}\text { effective gravitational field due to centrifugal } \\
\text { force }\end{array}$ & $Z$ & $\begin{array}{l}\text { axial coordinate (positive downstream); origin } \\
\text { at throat on nozzle centerline }\end{array}$ \\
\hline Hall & Hall & $z$ & $\begin{array}{l}\text { longitudinal coordinate (positive downstream, } \\
\text { along magnetic field); origin at plasma edge in }\end{array}$ \\
\hline$i$ & ion & & plasma-field mixing layer \\
\hline in & incoming & $1 / 4$ & one quarter \\
\hline kin & kinetic & $1 / 2$ & radial location halfway through width $\mathrm{d} R$ \\
\hline$L H$ & Lower Hybrid & I & constant from Section 2.3.3, Equation (96a) \\
\hline long & longitudinal & II & constant from Section 3.4, Equation (186) \\
\hline $\operatorname{mag}$ & magnetic & III & $\begin{array}{l}\text { denoting constant of integration from } \\
\text { Equation (C4) }\end{array}$ \\
\hline $\max 1$ & maximum bound & $\mathrm{IV}$ & 1enotino constant of inteoration from \\
\hline $\max 2$ & maximum bound (even larger) & IV & $\begin{array}{l}\text { denoting constant of integration from } \\
\text { Equation (C7) }\end{array}$ \\
\hline mid & middle of transition layer & $\mathrm{V}$ & denoting constant from Equation (G8b) \\
\hline$n$ & number density & VI & denoting constant from Equation (G14) \\
\hline norm & normalized & VII & denoting constant from Equation (G14) \\
\hline out & outgoing & $\beta$ & pertaining to global beta \\
\hline$p$ & plasma & $\Delta$ & maximum radial extent \\
\hline proj & projection & $\eta$ & resistive \\
\hline pe & plasma edge & $\delta$ & just beyond diffusive mixing layer \\
\hline
\end{tabular}




$\begin{array}{ll}\mu & \text { mobility } \\ \rho & \text { density } \\ \theta & \text { azimuthal } \\ \tau & \text { time } \\ \boldsymbol{\sigma} & \text { vorticity } \\ \perp & \text { perpendicular to local flux surfaces } \\ / / & \text { parallel to local longitudinal field } \\ \infty & \text { asymptotic }\end{array}$

\section{Superscripts}

E electric field

it ion trapping

$k \quad$ azimuthal wave number

$M_{\theta} \quad$ azimuthal mode number

$\begin{array}{ll}\text { ran } & \text { random } \\ \text { wave } & \text { wave-energy bound } \\ x & \begin{array}{l}\text { quasi-radial coordinate (positive toward wall); } \\ \text { origin at plasma edge in plasma-field mixing } \\ \text { layer }\end{array} \\ y & \begin{array}{l}\text { azimuthal coordinate (positive clockwise, } \\ \text { facing downstream); origin at plasma edge in } \\ \text { plasma-field mixing layer }\end{array} \\ \boldsymbol{\Delta} & \begin{array}{l}\text { variation radially outward } \\ \text { state of quantity as it exists in displaced } \\ \text { element }\end{array} \\ & \text { phase factor exponent } \\ (0) & \begin{array}{l}\text { Fourier-mode amplitude corresponding to a } \\ \text { zero-order small perturbation (equilibrium) }\end{array} \\ (1) & \begin{array}{l}\text { Fourier-mode amplitude corresponding to a } \\ \text { first-order small perturbation }\end{array} \\ & \text { moving reference frame of plasma }\end{array}$

ran random

quasi-radial coordinate (positive toward wall); origin at plasma edge in plasma-field mixing azimuthal coordinate (positive clockwise, facing downstream); origin at plasma edge in

state of quantity as it exists in displaced

Fourier-mode amplitude corresponding to a zero-order small perturbation (equilibrium)

Fourier-mode amplitude corresponding to a moving reference frame of plasma 


\section{Appendix B.-Influence of Dwell Time on Plasma-Field Mixing Layer Width}

Starting from Equation (99), and within the context of a quasi-one-dimensional model, it can be shown without further approximation that the square of the interface width, undergoing resistive diffusion along the flow, can be expressed as follows:

$$
\delta_{t}^{2}=\frac{\rho_{b}}{\rho_{t}} \frac{D_{t} L_{\text {back }}}{V_{t}} \int_{0}^{L_{\text {back }}} \frac{D(\varsigma)}{D_{t}} \frac{\rho(\varsigma)}{\rho_{b}} \frac{A(\varsigma)}{A_{t}} \frac{\mathrm{d} \varsigma}{L_{\text {back }}}
$$

It has been assumed that the initial interface width is zero. If not, one can add $\delta_{b}^{2}$ to the right-hand side of the above equation. In this equation, subscript $t$ refers to the position of the throat, and the integration proceeds back a distance, $L_{\text {back, }}$ to the breech of the nozzle. The breech position is represented by subscript $b$. The symbols $D, \rho, V$, and $A$ represent, respectively, the resistive diffusivity, plasma mass density, plasma flow velocity, and core plasma crosssectional area at position $\varsigma$ measured upstream from the throat at the position $\varsigma=0$.

If the resistivity were classical, then the quasi-onedimensional temperature dependence of $D$ on position $\zeta$ would be known along the flow. Likewise, the quasi-onedimensional density dependence $\rho(\zeta)$ is known along the flow. For a given nozzle shape (actually the shape of the confined plasma), the area dependence $A(\zeta)$ is known as well. All of these quantities can be obtained from an ideal magnetohydrodynamic (MHD) simulation or a quasi-onedimensional model. Their numerical representations can then be inserted into the above integral, which can subsequently be performed numerically to provide an estimate of the resistively broadened interface width at the position of the throat. Thus, an ideal MHD simulation or quasi-onedimensional model can be employed to make a preliminary estimate of the resistive interface broadening, by numerically evaluating a single integral.

In this appendix, however, we want to show that fairly close upper bounds to the above expression can be obtained in a simple manner, without having to perform the numerical integration.

It is assumed here that the relative-area variation of an annular ring containing the plasma-field mixing layer is the same as that of the main nozzle shape, a function of longitudinal distance along the flow. Conservation of mass flow is assumed $(\rho(\varsigma) V(\varsigma) A(\varsigma)=$ a constant), where $A(\varsigma)$ is the crosssectional area at position $\zeta$ and velocity $V(\varsigma)$ is primarily parallel to the core plasma Z-axis. From Equation (99), we see that if the resistive diffusivity is replaced by its larger value $D_{t}$ in the throat (since temperature drops along the flow and there is classical resistivity), the square of the maximum value of the mixing layer $\delta_{\max 1}^{2}$ can be bound at the throat by

$$
\delta_{\max 1}^{2}=D_{t} \int_{0}^{t_{b t}} \mathrm{~d} t=-D_{t} \int_{t_{b t}}^{0} \mathrm{~d} t=D_{t} \int_{0}^{L_{\text {back }}} \frac{1}{V(\varsigma)} \mathrm{d} \varsigma
$$

Here, we integrate backwards from the throat $t$ to the breech $L_{\text {back }}$ using a quasi-one-dimensional model. The longitudinal coordinate $\zeta$ runs from 0 in the throat to $L_{\text {back }}$ in the breech. The outgoing longitudinal velocity $V(\zeta)>0$ is assumed to be essentially the same in the plasma-field mixing layer as in the core plasma, in virtue of the above statement about relative area variation in the annulus containing the plasma-field mixing layer.

Now, by quasi-one-dimensional mass conservation, $V(\varsigma)=\left[\rho_{t} V_{t} A_{t}\right] /[\rho(\varsigma) A(\varsigma)]$. A still larger bound on $\delta_{\max 2}$ can be obtained by replacing $V$ in the above integral by a lower bound. This is obtained by replacing $\rho(\zeta)$ by the larger density in the breech $\rho_{b}$ in the above expression for the longitudinal flow velocity $V(\varsigma)$. Then the expression in Equation (D2) is bounded above by

$$
\delta_{\max 2}^{2}=D_{t}\left(\frac{\rho_{b}}{\rho_{t} V_{t}}\right) \int_{0}^{L_{\text {back }}}\left[\frac{A(\zeta)}{A_{t}}\right] \mathrm{d} \zeta
$$

For the relative area variation, we take the following generic model. The radius of the nozzle as a function of longitudinal position measured from the throat at position $\varsigma=0$ is taken as

$$
r(\varsigma)=r_{t}\left[1+\left.\left(\frac{\varsigma}{\varsigma_{\text {area }}}\right)^{2}\right|^{1 / 2}\right.
$$

where $\zeta_{\text {area }}$ is a characteristic axial length over which significant radial variation of the nozzle area occurs. (The length $\zeta_{\text {area }}$ is not exactly the same as the distance $L_{\text {back }}$ from throat to breech.) Obviously, $\mathrm{d} r / \mathrm{d} \zeta=0$ at $\varsigma=0$, which is appropriate for the shape of the throat. Therefore, the relative area variation is

$$
\frac{A(\varsigma)}{A_{t}}=1+\left(\frac{\varsigma}{\varsigma_{\text {area }}}\right)^{2}
$$

Use of Equation (B5) in Equation (B3) yields 


$$
\delta_{\max 2}^{2}=D_{t}\left(\frac{\rho_{b}}{\rho_{t} V_{t}}\right) \varsigma_{\text {area }}\left[\left(\frac{L_{\text {back }}}{\text { Sarea }_{\text {area }}}\right)+\frac{1}{3}\left(\frac{L_{\text {back }}}{\text { Sarea }_{\text {are }}}\right)^{3}\right]
$$

A radius contraction by a factor 2 from breech to throat is reasonable, producing density and velocity functions of $\varsigma$ up to the throat that differ only by a few percent from what would be produced by an infinite contraction ratio. From Equation (B5), an area contraction of 4 implies that $L_{\text {back }} / \varsigma_{\text {area }}=\sqrt{3}$. Then Equation (B6) becomes

$$
\begin{aligned}
\delta_{\max 2}^{2}=D_{t}\left(\frac{\rho_{b}}{\rho_{t} V_{t}}\right)\left(\frac{L_{\mathrm{back}}}{\sqrt{3}}\right)(2 \sqrt{3}) & \\
= & D_{t}\left(\frac{\rho_{b}}{\rho_{t} V_{t}}\right) 2 L_{\text {back }}
\end{aligned}
$$

Now, $\rho_{b} / \rho_{t} \approx 3 / 2$ constitutes a very good approximation to the nozzle flow results (see Eq. (10)). Then the upper bound becomes

$$
\delta_{\max 2}^{2} \approx D_{t}\left(\frac{3}{V_{t}}\right) L_{\mathrm{back}}
$$

Thus, for a radius-contraction ratio of 2 , the resistive layer width at the throat is bounded above by a quantity that is larger than the original estimate, $\sqrt{\left[D_{t} L_{b a c k} / V_{t}\right]}$, by only a factor of about $\sqrt{3}=1.7$. For a radius-contraction ratio of 3 , the numerical factor proves to be 2.3 . 


\section{Appendix C.-Planar Similarity Solution for Resistive Diffusion of Field Into Plasma}

When cylindrical effects are not important (thin plasmafield mixing layer), Equation (90c) can be written as follows:

$$
\partial_{X}^{2} B=\partial_{\tau} B
$$

Here, $B=B_{z} ; X$ is the inward radial distance from the plasma edge; and $\tau=\left[(z D) / V_{z}\right]$, where $z$ is the local axial coordinate, $D$ is the uniform resistive diffusivity of plasma, and $V_{z}$ is the uniform axial velocity of plasma. Note that Equations (90) and (C1) take the point of view of describing the process of magnetic-field diffusion into the plasma within the lab frame, in which a steady state (with flow) exists. Boundary conditions on the system are envisioned to be applied at the breech end and will be discussed below.

One-dimensional diffusion equations of this type permit similarity solutions having the form $B(X, \tau)=\mathrm{f}(u)$, wherein $u$ is the similarity variable, $u=X^{2} / \tau$. The equation for $\mathrm{f}(u)$ reads

$$
f^{\prime \prime}(u)=-\left(\frac{1}{4}\right) f^{\prime}(u)\left(1+\frac{2}{u}\right)
$$

where prime symbols denote derivatives with respect to the argument of the function.

Defining $g(u)=f^{\prime}(u)$, the equation for $g(u)$ is

$$
\frac{\mathrm{d}[\ln g(u)]}{\mathrm{d}(u / 4)}+\frac{\mathrm{d}\left(\frac{u}{4}+\ln \sqrt{\frac{u}{4}}\right)}{\mathrm{d}(u / 4)}=0
$$

The solution of Equation (C3) for $\mathrm{g}(u)$ is immediate. One then has

$$
f^{\prime}(u)=C_{\Pi I}\left(\frac{1}{\sqrt{u}}\right) e^{-u / 4}
$$

where $C_{\mathrm{III}}$ is a constant of integration. Integrating Equation (C4) from $u=\infty$ to $u$, one has

$$
\mathrm{f}(u)-\mathrm{f}(\infty)=C_{\mathrm{III}}\left[\int_{0}^{u} \frac{e^{-u / 4} \mathrm{~d} u}{\sqrt{u}}-\int_{0}^{\infty} \frac{e^{-u / 4} \mathrm{~d} u}{\sqrt{u}}\right\rceil_{\mid}
$$

though $f(\infty)$ corresponds to a field in the plasma at $z=0$. A boundary condition is that the fields have not yet diffused into the plasma at the breech of the nozzle. Thus, $f(\infty)=0$. It is also observed that

$$
\int_{0}^{u} \frac{e^{-u / 4} \mathrm{~d} u}{\sqrt{u}}=2 \sqrt{\pi} \operatorname{erf}(0.5 \sqrt{u})
$$

Then Equation (C5) becomes the following:

$$
f(u)=B=C_{\mathrm{IV}}\left[1-\operatorname{erf}\left(\frac{0.5 X}{\sqrt{u_{\tau}}}\right)\right]
$$

Here, $C_{\mathrm{IV}}$ is just another modified constant of integration, and the original variables have been reinserted. Also, recall that $u_{\tau}={ }_{z} D / V_{z}$. Note that the latest constant $C_{\mathrm{IV}}$ represents the uniform value of $B$ at the edge of the plasma, where $X=0$. 



\section{Appendix D.-Azimuthal Magnetic Field When Hall Voltage Is Shorted Out}

This appendix constitutes an estimate of the azimuthal magnetic field that is necessitated by the incoming longitudinal return current, which must balance the outgoing transverse Hall current if the Hall current circuit is fully closed. The estimate is admittedly non-self-consistent in the sense that results obtained by neglecting the azimuthal magnetic field are used to estimate the size of that field. The effects of plasma rotation and electron pressure are included here.

From Equations (62) and (67) the Hall current density can be written as

$$
J_{\text {Hall }}=-\Lambda^{-1} n q V_{\theta}=-\Lambda^{-1} B^{-1} \partial_{x} P_{i}
$$

All quantities herein are, in principle, functions of the distance along a given field line. In practice, they shall signify representative or average values near or in the throat region of the nozzle and near or in the plasma-field mixing layer.

Now, let $I_{\text {in }}$ represent the incoming longitudinal current, which will be presumed to exist inside the plasma core of radius $r(l)$. The region of the plasma-field mixing layer then begins at $r(l)$, and extends outwards by a small increment $\delta(l)$.

The total outgoing average Hall current $I_{\text {out }}$ can be roughly estimated as

$$
I_{\text {out }} \approx J_{\text {Hall }} 2 \pi r L
$$

where $r$ signifies the radius of the plasma core. The righthand side of Equation (D2) is intended to signify only average or representative values.

By setting $I_{\text {in }}=I_{\text {out }}$, the azimuthal magnetic field in or near the plasma-field mixing layer then can be estimated as follows:

$$
B_{\theta}=\frac{\mu_{0} I_{\text {in }}}{2 \pi r}=\mu_{0} J_{\text {Hall }} L=-\mu_{0} L \Lambda^{-1} B_{r Z}^{-1} \partial_{x} P_{i}
$$

Here, $B_{r} Z$ represents the original longitudinal magnetic nozzle field magnitude without any azimuthal component. This is equivalent to the projection of the total magnetic field vector into the r,Z-plane, Now, by making the convenient approximation that $\partial_{x} P_{i} \approx-P_{i} / \delta$, the ratio $B_{\theta} / B_{r Z}$ can be estimated. Also recall the definition of the ion $\beta_{i}$, namely that $\beta_{i}=2 \mu_{0} P_{i} / B^{2}$. Dividing Equation (D3) by the magnetic field $B_{r Z}$ one then finds

$$
\frac{B_{\theta}}{B_{r Z}}=\frac{L}{2 \Lambda}\left(\frac{2 \mu_{0} P_{i}}{B_{r Z}^{2}}\right) \delta^{-1}
$$

Recalling Equation (65) for $\Lambda$, this ratio of fields becomes

$$
\frac{B_{\theta}}{B_{r Z}}=\frac{\beta_{i}}{2}\left[\left.\frac{V_{/ l}}{\omega_{c i} \delta}\right|_{]}\right.
$$

Since the longitudinal flow velocity $V_{/ /}$is on the order of the ion thermal velocity, this result is approximately

$$
\frac{B_{\theta}}{B_{r Z}}=\frac{\beta_{i}}{2}\left[\left.\frac{a_{i}}{\delta}\right|_{]}\right.
$$

Suppose $\delta$ is scaled by the ion thermal gyroradius $a_{i}$, as in Equation (74). For example, suppose that $\delta$ is one ion gyrodiameter. The azimuthal magnetic field ratio is then on the order of $0.25 \beta_{i}$, whereas with the resistive plasma-field mixing layer, as in Equation (77), $\delta$ could be somewhat larger, and hence the azimuthal field would be somewhat smaller.

It is worth noticing that when the azimuthal magnetic field is somewhat comparable to the magnetic nozzle field, as is indicated here, then it is possible that substantial magnetic shear can arise within the plasma-field mixing layer. The magnetic nozzle field increases from a relatively small value within the plasma core to its ambient value in a rather short radial distance, which is the plasma-field mixing layer. It is well known that magnetic shear has a stabilizing influence on some microinstabilities as well as on flute-type instabilities driven in regions of adverse curvature of the magnetic field lines. For this reason among others it appears worth exploring in some detail whether the full Hall current mode of operation (including plasma rotation) may enhance the integrity of the plasma-field mixing layer. This is further developed in Appendix E. 



\section{Appendix E.-Inward Radial Drift of Propellant}

Although the main concern of this report is loss of propellant outward onto the nozzle magnetic field lines, it is worth pointing out that there also is a mechanism for inward drift, which is activated by the presence of the azimuthal magnetic field in a resistive plasma medium. For simplicity we assume a straight, cylindrical geometry.

The azimuthal magnetic field lines must enclose the core where, in a steady state, the incoming longitudinal current balances the transversely outgoing Hall current. The resulting small inward drift may be somewhat beneficial in maintaining the integrity of the core plasma. For simplicity, it was not included in the model of field diffusion into the plasma. It must arise, however, from a steady-state balance between the rate at which magnetic energy is resistively dissipated by the longitudinal current and the rate at which magnetic energy is brought into the core. This balance can be expressed as follows:

$$
\left(\frac{B_{\theta}^{2}}{2 \mu_{0}}\right) V_{r} 2 \pi r \mathrm{~d} Z=\eta J_{Z}^{2} \pi r^{2} \mathrm{~d} Z
$$

Here, it is known that in highly conducting plasma, magnetic flux can only be brought towards the core by the inward velocity of plasma; that is, the flux lines move inwards approximately with the plasma. Equation (E1) is essentially an integral form of Poynting's theorem.

Now, from Ampere's law, $B_{\theta}=\left(\mu_{0} I\right) /(2 \pi r)$, where $I$ is the longitudinal current within the core of radius $r$. Also, we have assumed uniform resistivity and uniform density of longitudinal current, $J_{Z}=I / \pi r^{2}$. As a result, one finds from Equation (E1) that the radial inward velocity at the edge of the core is

$$
V_{r}=\frac{4 D}{r}
$$

where $D=\eta / \mu_{0}$ is the resistive diffusivity of plasma.

Consider an example with classical resistivity (Spitzer resistivity) in hydrogen plasma with the parameters in Table I. The resistive diffusivity then is $D_{\mathrm{cl}}=3 \mathrm{~m}^{2} / \mathrm{s}$. Also, taking the core radius to be $r=0.1 \mathrm{~m}$, the inward drift velocity then is $120 \mathrm{~m} / \mathrm{s}$, which is 3 orders of magnitude smaller than the longitudinal flow velocity of propellant. This inward drift velocity is somewhat comparable to the outward diffusion velocity found earlier (see the discussion following Eq. (17)). 



\section{Appendix F.-Derivation of Some Results Noted in Presentation of Initial Boundary-Layer Width}

The results presented below in Sections F.1 and F.2 refer to the small electron displacement distances treated in the discussion around Equations (121) and (122) in Section 3.1, "Interface Width in Breech of Nozzle." Numerical factors of order 1 will be suppressed in the derivations represented here. Section F.3 pertains to small inertial forces on individual ions (see Eq. (103)). Section F.4 validates the assumption when only electrons carry azimuthal current.

\section{F.1 Radial Mobility Drift Distance of Electron Versus $c / \omega_{p i}$}

In Equation (122), the first term gives the outward quasiradial mobility drift-velocity of the electron fluid in the electric field $E_{x}$ in the limit of large $\Omega=\left(\omega_{c e} / v_{e}\right)$. This quasi-radial velocity term will be multiplied by about onefourth of an ion gyroperiod $\left(\approx 1 / \omega_{c i}\right)$ so as to obtain the outward quasi-radial distance $x_{\mu}$ drifted by electrons during that time increment.

$$
\begin{aligned}
x_{\mu}=\frac{1}{\Omega} \frac{E_{x}}{B_{z}} \frac{1}{\omega_{c e}} \frac{\omega_{c e}}{\omega_{c i}}=\frac{1}{\Omega} \frac{J_{y} B_{z}}{n q B_{z}} & \frac{1}{\omega_{c e}} \frac{m_{i}}{m_{e}} \\
& =\frac{1}{\Omega} \frac{\mu_{0} J_{y}}{n q \mu_{0}} \frac{1}{\omega_{c e}} \frac{m_{i}}{m_{e}}
\end{aligned}
$$

Ampere's law is used in the numerator of the last expression above, to replace $\mu_{0} J_{y}$ by $[\nabla \times \mathbf{B}]_{y}$. Then, the radial length increment in the definition of $[\nabla \times \mathbf{B}]_{y}$ is identified as $c / \omega_{p}$. After substituting the definition of $\omega_{c} e=q B / m_{e}$, a resulting combination of factors is identified as the square of the electron plasma frequency $\omega_{p e}^{2}=n q^{2} / \varepsilon_{0} m_{e}$ in mks units. Finally, recall the relation $\varepsilon_{0} \mu_{0}=1 / c^{2}$, where $c$ is the speed of light. After some cancellation, and using $\omega_{p e}=\omega_{p i} \sqrt{m_{i} / m_{e}}$, the above expression reduces to

$$
x_{\mu}=\frac{1}{\Omega} \frac{c}{\omega_{p i}}
$$

Thus the classical outward mobility drift distance traversed by an electron in the space-charge quasi-radial electric field is small compared with the initial outward ion penetration depth.

\section{F.2 Radial Diffusion Distance of Electron Versus $c / \omega_{p i}$}

We consider the quasi-radial diffusion distance $x_{D}$ traversed by an electron undergoing a random walk (of gyroradius steps) across the magnetic field, during one-fourth of an ion gyroperiod. Recall that the diffusion coefficient of an electron in a magnetic field is given by the expression $D_{e}=a_{e}^{2} / \tau_{e}=a_{e}^{2} v_{e}$, wherein $v_{e}$ is the collision frequency of an average electron (having $\omega_{c e} \gg v_{e}$ ) and $a_{e}$ is the electron thermal gyroradius. Then

$$
x_{D}=\sqrt{D_{e} t}=\sqrt{a_{e}^{2} \frac{v_{e}}{\omega_{c e}} \frac{\omega_{c e}}{\omega_{c i}}}=\sqrt{\frac{a_{e}^{2}}{\Omega} \frac{m_{i}}{m_{e}}}=\sqrt{\frac{a_{i}^{2}}{\Omega}}=\frac{a_{i}}{\sqrt{\Omega}}
$$

but the ion thermal gyroradius $a_{i}$ is comparable to the ion inertia length $c / \omega_{p i}$ whenever the ion $\beta$ is on the order of 1 (as expected according to Appendix $\mathrm{H}$ ). Hence,

$$
x_{D} \approx \Omega^{1 / 2} c / \omega_{p i}
$$

Thus the classical outward collisional diffusion traversed by an electron across the local magnetic field is small compared with the initial outward ion penetration depth.

\section{F.3 Fluid Deceleration Neglected in Ion Trajectory Equation}

An equivalent gravitational deceleration field was neglected in the trajectory equation of an ion, which was employed in Section 3.2, "Linear Theory of Lower Hybrid Drift (LHD) Instability." This force field is to be compared to the force on the ion from the radial space-charge electric field.

From Equation (H11) and by recalling that the square of the Alfven velocity is $V_{A}^{2}=B^{2} / \mu_{0} \rho$, we estimate that $g_{\text {eff }} \delta=h V_{x} V_{A}$. Here $V_{x}$ is the radial fluid expansion velocity of the plasma during the first one-fourth ion gyroperiod, and $h$ is the fraction of that velocity that is lost to deceleration during that time increment. Setting $\delta=c / \omega_{p i}=V_{A} / \omega_{c i}$ (the second equation is an identity), the effective gravitational acceleration becomes

$$
g_{\text {eff }}=h V_{x} \omega_{c i}
$$


which is approximately the same as Equation (H11). Recall that $c$ is the speed of light, $\omega_{p i}$ is the ion plasma frequency, and $\omega_{c i}$ is the ion gyrofrequency in the plasma-field mixing layer. Because $\omega_{c i}=q B / m_{i}$, the equivalent gravitational force on the ion is

$$
m_{i} g_{\text {eff }}=h q V_{x} B
$$

The radial momentum equation for fluid electrons (neglecting electron pressure for simplicity) is $n q E_{x}=\mathrm{J}_{y} B_{z}$. With help of Ampere's law, this becomes

$$
q E_{x}=-\frac{1}{n} \partial_{x}\left(\frac{B^{2}}{2 \mu_{0}}\right) \approx \frac{m_{i} V_{A}^{2}}{c / \omega_{p i}}
$$

We have ignored a sign to just get the order of magnitude and have set the length scale for magnetic pressure change to $c / \omega_{p i}$. Using the identity mentioned above, radial force on the ion (magnitude only) due to the space-charge field is found to be

$$
q E_{x}=q V_{A} B
$$

This electric force exceeds $m_{i} g_{\text {eff }}$ when the plasma-fluid's radial expansion velocity is sub-Alfvenic.

\section{F.4 Azimuthal Current Carried by the Ions During One-Fourth of a Gyroperiod}

A fundamental assumption in Section 3.1 has been that only the electrons carry azimuthal current during the short time $\left(1 / \omega_{c i}\right)$ when a volume element of plasma first runs into the confining external magnetic field. In this subsection, we test that assumption. Again neglecting electron pressure for simplicity, it is seen from the above estimate of the radial space-charge electric field that the azimuthal $\mathbf{E} \times \mathbf{B}$ drift velocity of the electrons is just the Alfven velocity. If the azimuthal fluid velocity of the ions were much smaller than that in the early-time increment of interest, then the ion contribution to the azimuthal current also would be small. The azimuthal electric field from the radial fluid expansion is $E_{y}=V_{x} B_{z}$. The Faraday phase due to time-dependent interior flux expulsion by the instantaneous electron current layer is assumed already completed. The azimuthal velocity acquired by an ion during time $\tau_{i} \approx 1 / \omega_{c i}$ is then

$$
V_{y i} \approx q E_{y} \tau_{i} / m_{i}=q E_{y} / m_{i} \omega_{c i}=E_{y} / B_{z}=V_{x i}
$$

This is small compared with the electron-current velocity when the plasma fluid crossfield expansion is sub-Alfvenic. 


\section{Appendix G.-Effect of Electron Pressure on the Initial Plasma-Field Mixing Layer Width}

This appendix examines the effect of electron pressure on the initial width of the plasma-field mixing layer. The effect of electron collisions has been sufficiently covered in the main text. For conciseness, we will ignore electron collisions here. However, electron (and ion) pressure $\left(P_{e} \approx P_{i}\right)$ will be included in this calculation of the initial width of the plasmafield mixing layer. Moreover, we attempt to carry out a more rigorous and detailed evaluation of the radial average electric field needed in this calculation, yet without invoking specific shapes for the profiles of density and magnetic field within the plasma-field mixing layer.

Consider the azimuthal frame of reference in which newly injected ions carry no macroscopic azimuthal current. This is presumably the lab frame. In that frame, the quasi-radial component (i.e., directed across flux surfaces) of the electron-fluid momentum equation then reads as follows:

$$
\partial_{x} P_{e}=-n q\left(E_{x}+V_{y} B_{z}\right)
$$

A key feature of the model is that, for times less than an ion gyroperiod the azimuthal current density $J_{y}=-n q V_{y}$ is carried only by the electrons. As discussed in the main text, we shall assume that quasi-neutrality has been achieved in an early transient subphase and is maintained (see Section 3.1, "Interface Width in Breech of Nozzle"). We also assume that the electron and ion temperatures are equal and uniform within the plasma-field mixing layer.

Solving Equation (G1) for the space-charge electric field and using Ampere's law on $J_{y}$,

$$
E_{x}=-\frac{1}{n q_{e}} \partial_{x}\left(\frac{B^{2}}{2 \mu_{0}}+\frac{P_{\text {tot }}}{2}\right)
$$

Here, $P_{\text {tot }}=P_{e}+P_{i}=2 P_{i}$ is the total plasma pressure. Therefore, with mass density $\rho=m_{i} n$, it is obvious that

$$
-\frac{2 q_{e} \Delta}{m_{i}}\left\langle E_{x}\right\rangle=\Delta\left\langle\frac{1}{\rho} \partial_{x}\left(\frac{B^{2}}{\mu_{0}}+P_{\text {tot }}\right)\right\rangle
$$

where $\Delta$ is the penetration depth of ions into confining magnetic field in the breech. The brackets $\langle\ldots .$.$\rangle signify the radial$ average of the enclosed quantity over the width of the plasma-field mixing layer.

Use of azimuthal momentum balance Equation (110) for the velocity of an individual ion at the farthest radial extent of the ion's trajectory, $v_{y i \Delta}=-1 / 2 \omega_{c i} \Delta$, allows the total energy balance of that ion, from Equation (111b) to be expressed as follows (the ion gyrofrequency, $\omega_{c i}$ without brackets, refers to the outer magnetic field beyond the plasma-field mixing layer):

$$
v_{x \operatorname{core} i}^{2}=-\frac{2 q_{e} \Delta}{m_{i}}\left\langle E_{x}\right\rangle+\frac{1}{4} \omega_{c i}^{2} \Delta^{2}
$$

Here, $v_{x}$ core $i$ is the radial velocity with which the ion is incident upon the edge boundary layer. It has been assumed that the incident azimuthal velocity $v_{y \text { core } i}=0$ for a representative ion in the midst of a thermal distribution.

The intent is to utilize Equation (G3) in Equation (G4). Therefore, the two radial averages indicated in Equation (G4) are evaluated. We begin with the second term because it entails the most straightforward evaluation. Using $P_{\text {tot }}=2 n T$ with uniform $T$, the second term is

$$
\Delta\left\langle\frac{1}{n m_{i}} \partial_{x} P_{\text {tot }}\right\rangle=\Delta\left\langle\frac{2 T}{m_{i}} \frac{\partial_{x} n}{n}\right\rangle=\frac{2 T}{m_{i}} \Delta\left\langle\partial_{x} \ln n\right\rangle
$$

The notation $V_{t h i}^{2}=2 T / m_{i}$ is introduced for the square of the ion thermal velocity. The radial average of the partial derivative of the logarithm of number density is evaluated as follows:

$$
\begin{aligned}
\left\langle\partial_{x} \ln n\right\rangle=\frac{1}{\Delta} \int_{\text {core }}^{\Delta} \partial_{x} \ln n \mathrm{~d} x & =\frac{1}{\Delta}\left[\ln n^{\Delta}-\ln n_{\text {core }}\right] \\
& =\frac{1}{\Delta} \ln \frac{n^{\Delta}}{n_{\text {core }}}=-\frac{1}{\Delta} \ln \frac{n_{\text {core }}}{n^{\Delta}}
\end{aligned}
$$

This is to be multiplied by $\Delta$ in Equation (G5). It is reasonable to associate the characteristic width $\Delta$ of the plasma-field mixing layer with the $1 / e$ spatial decay length of the plasma density (where $e \approx 2.71828$, the base of natural logarithms). The characteristic width $\Delta$ originally was associated with the protrusion of an average ion's trajectory beyond the core plasma into the magnetic field region. Its quantitative connection with the density profile's spatial decay, as well as its connection with the spatial increase of the magnetic pressure, requires more calculation than can be accommodated here. This clarification would require following the full thermal velocity distribution of trajectories of outgoing ions into the (unknown) nonuniform magnetic field profile. But using a few $\Delta \mathrm{s}$ for the $1 / e$ spatial decay of the plasma density, say $n=n_{\text {core }} \exp \left(-x / C_{\mathrm{V}} \Delta\right)$, makes little difference, as shown later. Then $\ln \left(n_{\text {core }} / n^{\Delta}\right)=1 / C_{\mathrm{V}}$, where $n^{\Delta}=n_{\text {core }} / e$ is 
the variation of number density radially outward and $C_{\mathrm{V}}$ is an unknown free constant. Note, however, that the use of this specific decaying exponential density profile is only an example; it is not essential to the argument. Consequently,

$$
\left\langle\partial_{x} \ln n\right\rangle=-\frac{1}{\Delta}
$$

If the spatial decay length of the density were taken as $C_{\mathrm{V}} \Delta$, then instead of Equation (G7a) one would find

$$
\left\langle\partial_{x} \ln n\right\rangle=-\frac{1}{C_{\mathrm{V}} \Delta}
$$

Use of Equation (G7a) in Equation (G5) yields

$$
\Delta\left\langle\frac{1}{\rho} \partial_{x} P_{\mathrm{tot}}\right\rangle=-V_{\mathrm{th} i}^{2}=-\frac{2 T}{m_{i}}
$$

Retaining the flexibility of $C_{\mathrm{V}} \Delta$ for the characteristic distance of spatial decay of the density profile, Equation (G8a) becomes instead

$$
\Delta\left\langle\frac{1}{\rho} \partial_{x} P_{\mathrm{tot}}\right\rangle=-\frac{V_{\mathrm{th} i}^{2}}{C_{\mathrm{V}}}
$$

We have assumed that the characteristic length for the increase of magnetic pressure in the plasma-field mixing layer $B^{2} / 2 \mu_{0}$ is $\Delta$. The quantitative connection of the density decay length to $\Delta$ has not been established, so a few values $C_{\mathrm{V}} \neq 1$ are considered.

Now the second term on the right-hand side in Equation (G3) has been accounted for. Next, we address the first term in Equation (G3). A simple calculation in terms of the local Alfven velocity $V_{A}$ yields

$$
\Delta\left\langle\frac{1}{\rho} \partial_{x} \frac{B^{2}}{\mu_{0}}\right\rangle=\Delta\left\langle\partial_{x} V_{A}^{2}+V_{A}^{2} \frac{\partial_{x} n}{n}\right\rangle
$$

Recall that the local Alfven speed squared is $V_{A}^{2}=B^{2} / \mu_{0} \rho$. The first term of Equation (G9) can be written

$$
\begin{aligned}
\Delta\left\langle\partial_{x} V_{A}^{2}\right\rangle=\Delta \frac{1}{\Delta} \int_{\text {core }}^{\Delta} & \partial_{x} V_{A}^{2} \mathrm{~d} x \\
& =V_{A \Delta}^{2}-V_{A \text { core }}^{2} \approx V_{A \Delta}^{2}=e V_{A}^{2}
\end{aligned}
$$

Subscript " $\Delta$ " refers to local evaluation at the outer edge of the plasma-field mixing layer, and subscript "core" refers to evaluation at its inner (plasma core) edge. But the final $V_{A}$ without those subscripts is to be evaluated with the outer $B$ and the inner $\rho$. The inner $\rho$ is taken as $e$ multiplied by the outer $\rho$. The density's spatial decay length is taken as the original $\Delta$.

If the spatial decay length of the density were instead taken to be $C_{\mathrm{V}} \Delta$, then instead of Equation (G10a) one would find

$$
\Delta\left\langle\partial_{x} V_{A}^{2}\right\rangle=e^{1 / C_{\mathrm{V}}} V_{A}^{2}
$$

Next, the second term of Equation (G9) is evaluated just by using an intermediate value of the local $V_{A}^{2}$. Since $B$ increases going outwards and $\rho$ decreases, $V_{A}^{2}$ increases strongly going outwards. Therefore, its intermediate value is simply taken as half the outer value. For the density decay scale of $\Delta$, one finds

$$
\Delta\left\langle V_{A}^{2} \frac{\partial_{x} n}{n}\right\rangle=-\frac{1}{2} e V_{A}^{2}
$$

The local $V_{A}^{2}$ appears in Equation (G11a), and the final $V_{A}^{2}$ in Equation (G10a) has been evaluated at the outer $B$ and the inner $\rho$.

If the spatial decay length of the density were $C_{\mathrm{V}} \Delta$, one would find instead

$$
\Delta\left\langle V_{A}^{2} \frac{\partial_{x} n}{n}\right\rangle=-\frac{1}{2} e^{1 / C_{\mathrm{V}}} \frac{1}{C_{\mathrm{V}}} V_{A}^{2}
$$

Use of Equations (G7a) to (G11a) in Equation (G3) yields

$$
-\frac{2 q \Delta}{m_{i}}\left\langle E_{x}\right\rangle=\frac{e}{2} V_{A}^{2}\left[1-\left.\frac{\beta_{G}}{e}\right|_{j}\right.
$$

The global beta, $\beta_{G}$, is the ratio of core plasma pressure (electron pressure plus ion pressure) to outer magnetic pressure. Since $\beta_{G}=1$ for pressure balance across a static layer, the right-hand side is guaranteed to be positive, in agreement with $E_{x}$ being negative. In case of radial deceleration of the macroscopic plasma fluid, it will be shown that $\beta_{\mathrm{G}}<1$, making the right-hand side of Equation (G12a) still more positive.

Equation (G12a) applies to the case that the density decay length in the plasma-field mixing layer is only $\Delta$. If instead it were $C_{\mathrm{V}} \Delta$, then Equation (G12a) would be replaced by

$$
\begin{aligned}
& -\frac{2 q \Delta}{m_{i}}\left\langle E_{x}\right\rangle \\
& \left.\quad=e^{1 / C_{\mathrm{V}} V_{A}^{2} \frac{2 C_{\mathrm{V}}-1}{2 C_{\mathrm{V}}}\left[1-\beta_{G} \frac{1}{e^{1 / C_{\mathrm{V}}}\left(2 C_{\mathrm{V}}-1\right)}\right]}\right]
\end{aligned}
$$


For the negative space-charge field $E_{x}$ it is clearly necessary that $C_{\mathrm{V}}>1 / 2$, but this is not sufficient because the expression in brackets in Equation (G12b) also must be positive. The latter condition amounts to

$$
c_{\mathrm{V}}>1 / 2\left[1+\left.\beta_{G} e^{-1 / c_{\mathrm{V}}}\right|_{]}\right.
$$

It will be shown below that $\beta_{G} \leq 1$. Then this condition is satisfied for $C_{\mathrm{V}} \geq 0.5925$. The lower bound 0.5925 for $C_{\mathrm{V}}$ is an artifact of shortening the electron pressure drop for fixed magnetic pressure gradient. The radial electron momentum equation reads: $n_{e} q E_{x}=-\partial_{x} P_{e}-\partial_{x}\left(B^{2} / 2 \mu_{0}\right)$. Thus, an artificially sharp negative electron pressure gradient, for fixed positive magnetic pressure gradient, annihilates the spacecharge electric field, an unphysical consequence.

Now Equations (G12a) and (G12b) are used to calculate the plasma-field mixing layer thickness $\Delta$, following the same procedures as are utilized in the main text. Both Equations (G12a) and (G12b) can be expressed as

$$
-\frac{2 q \Delta}{m_{i}}\left\langle E_{x}\right\rangle=C_{\mathrm{VI}} V_{A}^{2}\left(1-C_{\mathrm{VII}} \beta_{G}\right)
$$

where $C_{\mathrm{VI}}$ and $C_{\mathrm{VII}}$ are constants:

$$
\begin{gathered}
C_{\mathrm{VI}}=e^{1 / C_{\mathrm{V}} \frac{2 C_{\mathrm{V}}-1}{2 C_{\mathrm{V}}}} \\
C_{\mathrm{VII}}=\frac{1}{e^{1 / C_{\mathrm{V}}}\left(2 C_{\mathrm{V}}-1\right)}
\end{gathered}
$$

The $\Delta$ represents the physical penetration distance of a representative ion at the edge of the plasma into the confining magnetic field, when that element of plasma first encounters the interface region. The nondimensional version of $\Delta, \Delta_{\text {norm }}$, is represented as

$$
\Delta_{\text {norm }}=\frac{\Delta \omega_{p i}}{c}
$$

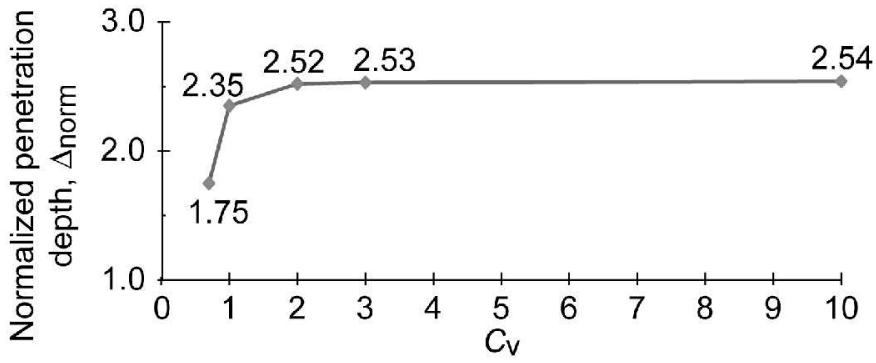

Figure 6.-Dependence of normalized ion penetration depth on constant of the density decay length, $C_{\mathrm{v}} \Delta$. in which $\omega_{p i}$ is the ion plasma frequency referred to the ion number density in the core. Using the same procedures as in the main text, namely Equation (117), $\Delta_{\text {norm }}^{2}=\left(\Delta \omega_{p i} / c\right)^{2}$, and the subsequent discussion, one finds

$$
\Delta_{\text {norm }}^{2}=\frac{\pi^{2} / 4}{1-\pi^{2} / 16} C_{\mathrm{VI}}\left(1-C_{\mathrm{VII}} \beta_{G}\right)
$$

Inserting the constants from Equation (G15) into Equation (G17), we then find

$$
\Delta_{\text {norm }}^{2}=\frac{\pi^{2} / 4}{1-\pi^{2} / 16} \frac{1}{2 C_{\mathrm{V}}}\left[e^{1 / C_{\mathrm{V}}}\left(2 C_{\mathrm{V}}-1\right)-\left.\beta_{G}\right|_{]}\right.
$$

Setting the global beta to its upper bound $\beta_{G}=1$ in Equation (G18), the lower bounds can be found for the numerical values of $\Delta_{\text {norm }}$. We say that these results are "lower bounds" for the following reason: In Appendix $\mathrm{H}$, we show that $\beta_{G}=1$ in the absence of radial deceleration of the plasma by the magnetic field (i.e., no fluid-plasma motion across magnetic flux), but $\beta_{G}<1$ in the presence of radial deceleration ("radial" always means "quasi-radial" across the flux surfaces). Hence, according to Equation (G18), setting $\beta_{G}=1$ produces a value of $\Delta_{\text {norm }}$ that constitutes an underestimate in the presence of radial deceleration.

Figure 6 illustrates the dependence of nondimensional ion penetration depth $\Delta_{\text {norm }}$ into the external magnetic field on the characteristic electron density decay length into that field, $\left(C_{\mathrm{V}} \Delta\right)$, when $C_{\mathrm{V}}$ is regarded as an unknown free parameter. Figure 6 indicates that the (underestimated) width of the initial plasma-field mixing layer, as defined by the radial protrusion of a representative ion into the magnetic field, is quite insensitive to the exact numerical decay length assumed for the density halo. Here, $\beta_{G}$ has been replaced by its upper bound. Appendix $\mathrm{H}$ relates the numerical value of $\beta_{G}$ to the presence or absence of macroscopic radial deceleration of newly injected, radially expanding plasma. The effects of both electron and ion pressure gradients are explicitly included in Appendix $\mathrm{H}$. 



\section{Appendix H.-Relation Between Global Beta and Radial Deceleration}

Initially, displaced magnetic flux between the plasma and the metallic wall (behind the insulating layer) builds up external magnetic pressure to match the radially expanding internal plasma pressure and slow down the radial expansion. This phase constitutes a dynamical adjustment described below in more detail. It is to be distinguished from the subsequent resistive phase that consists of radially expanding ions attempting to drag reluctant electrons across magnetic flux. The latter phase may involve either classical resistivity or anomalous resistivity from microturbulence.

Newly injected high-temperature plasma tries to expand across the ambient magnetic field, if it is not injected right along the magnetic field lines. The preexisting nozzle magnetic field, however, counters that tendency early on. In the simplest MHD model, the high-conductivity Ohm's law is $\mathbf{E}+\mathbf{V} \times \mathbf{B}=0$, which has some validity at short times during the setting-up phase. Here, $\mathbf{E}$ is the electric field in the lab frame of reference. The symbol $\mathbf{V}$ is employed for the fluid (macroscopic) velocity vector of the plasma ions in order to be clearly distinct from the velocity vector $\mathbf{v}_{i}$ of an individual ion. As the plasma's lateral boundary crosses outwards through downstream-directed longitudinal magnetic flux (in the local z-direction), with quasi-radial fluid velocity $V_{x}$ (in the local $\mathbf{x}$-direction, $\hat{\mathbf{x}}=\hat{\boldsymbol{\theta}} \times \mathbf{B} / B$ ), an azimuthal electric field $\left(V_{x} B_{z}\right)$ is generated in the clockwise direction (looking downstream). Then, by Faraday's law (or Lenz's law),

$$
\oint \mathbf{E} \cdot \mathrm{d} \mathbf{l}=-\frac{\mathrm{d} \Theta}{\mathrm{d} t}
$$

where the closed-line integral is taken around the plasma circumference in the clockwise direction, and $\Theta$ is the enclosed longitudinal magnetic flux.

The downstream-directed magnetic flux thereby becomes diminished within the plasma and displaced to the annular volume outside of the plasma. Longitudinal magnetic flux can be laterally repositioned, but once established it cannot be created or destroyed within the volume defined by the highly conducting wall, during the short time interval of interest. The shifted flux, in addition to the original external flux, is squeezed between the plasma boundary and the conducting wall or coil, which thereby results in an increased magnetic field strength in the annulus outside of the plasma. The reconfigured flux therefore exerts an increased magnetic backpressure impeding the further radial expansion of plasma. The squeezed longitudinal magnetic flux acts like a compressible gas having an adiabatic index of 2 . The expanding plasma therefore experiences a radial deceleration.

Although the above brief description was based upon the simplest MHD model of a high-conductivity Ohm's law, it can be extended to more detailed plasma models. This model envisions neutral plasma motion across the magnetic field.
The kinetic ion intrusion $\Delta$ in the breech into the confining magnetic field takes place in a few hundredths of a microsecond in a field of thousands of Gauss. This takes place in a very short time span (on the order of one-quarter ion gyroperiod) compared with the transit time from breech to throat $\left(\sim 10 \mu \mathrm{s}\right.$ at a velocity of $\left.10^{5} \mathrm{~m} / \mathrm{s}\right)$. We assume this kinetic penetration has already occurred, and so we go to the next phase, which is described by a fluid model. For example, as a steady-flow configuration is approached, with axial symmetry, the azimuthal electric field in the lab frame tends to vanish; however, resistive currents are driven so as to continue to shield the plasma interior from penetration by the external magnetic field. To see this, use the next level of plasma model, namely, a resistive medium of resistivity $\eta$. In this case the perfect conductivity model $\mathbf{E}+\mathbf{V} \times \mathbf{B}=0$ evolves over time into $\mathbf{V} \times \mathbf{B}=\eta \mathbf{J}$, where $\mathbf{J}$ is the current density in the edge plasma (where $\mathbf{V}$ represents the resistive leakage of fluid velocity of the plasma in the quasiradial direction across the local magnetic field. The resistive currents generated according to this model are driven in the counterclockwise direction (looking downstream). They generate magnetic fields that have the net effect of excluding magnetic flux from the interior plasma but reenforcing it in the exterior annulus. Magnetic shielding by an edge layer of plasma current is according to Ampere's law, $\nabla \times \mathbf{B}=\mu_{0} \mathbf{J}$.

Still more detailed plasma models can be taken into account, in which collisions of electrons with ions are rare and the Hall effect is important. It is then necessary to include the effects of electric fields due to charge separation and to employ separate models for the electron and ion motions (see, for example, the discussion after Equation (101) in Section 3.1, "Interface Width in Breech of Nozzle"). Magnetic shielding of the interior plasma is again realized.

The purpose of this appendix is to focus on the initial dynamical adjustment phase, by furnishing an account of the influence of the radial deceleration of plasma on the $\beta_{G}$. We work in a reference frame that follows a plasma element in the main longitudinal flow, and a planar model of the plasma-field interface is employed. Curvature of the magnetic field lines is neglected for the short time interval of interest. The ion and electron quasi-radial momentum equations then can be expressed as follows:

$$
\rho\left(\partial_{t} V_{x}+V_{x} \partial_{x} V_{x}\right)+\partial_{x} P_{i}=n q E_{x}=-\partial_{x} \frac{B^{2}}{2 \mu_{0}}-\partial_{x} P_{e}
$$

The first equation is the ion quasiradial momentum balance equation, wherein the ions carry no azimuthal current at early times; the second equation, with the help of Ampere's law, is the electron quasiradial momentum balance equation. 
With the help of the ion mass conservation equation, $\partial_{t} \rho+\partial_{x}\left(\rho V_{x}\right)=0$, the equations in Equation (H2) may be combined as follows:

$$
\partial_{t}\left(\rho V_{x}\right)+\partial_{x}\left(\rho V_{x}^{2}\right)+\partial_{x}\left(P_{\text {tot }}+\frac{B^{2}}{2 \mu_{0}}\right)=0
$$

As mentioned above, $V_{x}$ is the radial component of the ionfluid velocity, and the equations are written in the frame that tracks the longitudinal flow. In Equation (H3), the total plasma pressure is $P_{\text {tot }}=P_{e}+P_{i}$. This equation would have had the same form were the ions to carry some azimuthal current.

Equation (H3) is integrated across the evolving interface plasma-field mixing layer of width $\delta$. The integration is performed from a point at the edge of the core plasma where the density is large (subscript "core") and the magnetic field is small, to just beyond the mixing layer where the density is small and the magnetic field is large (subscript " $\delta$ "). These inner and outer points are not fixed, but move radially with the plasma fluid.

The integral of the first term in Equation (H3) is rearranged with the time derivative moved in front of the whole integral. It then is important to note that contributions to that integral arise that are associated with the timedependent limits of integration. These exactly cancel the contributions to the integration that originate from the second term in Equation (H3). In view of these cancellations, the integrated equation then can be written as

$$
\langle\rho\rangle g_{\text {eff }} \delta=\left(\frac{B_{\delta}^{2}}{2 \mu_{0}}-P_{\text {core }}\right)
$$

In Equation (H4), $\langle\rho\rangle$ is the average mass density in the layer of width $\delta$, and the effective gravitational acceleration $g_{\text {eff }}$ is

$$
g_{\text {eff }}=-\frac{\mathrm{d}\left\langle V_{x}\right\rangle}{\mathrm{d} t}
$$

where $\left\langle V_{x}\right\rangle$ is the density-weighted average fluid velocity in the layer. Specifically,

$$
\begin{gathered}
\left\langle V_{x}\right\rangle=\frac{\int_{\text {core }}^{\delta} \rho V_{x} \mathrm{~d} x}{\int_{\text {core }}^{\delta} \rho \mathrm{d} x} \\
\langle\rho\rangle=\frac{1}{\delta} \int_{\text {core }}^{\delta} \rho \mathrm{d} x
\end{gathered}
$$

Now dividing through in Equation (H4) by the external magnetic pressure, the global beta is

$$
\beta_{G}=1-\frac{\langle\rho\rangle g_{\text {eff }} \delta}{B_{\delta}^{2} / 2 \mu_{0}}
$$

If there were no radial deceleration $(g=0)$ or radial macroscopic expansion $\left(V_{x}=0\right)$, then one would have $\beta_{G}=1$. With radial deceleration $g_{\text {eff }}>0$, one must have $\beta_{G}<1$. We shall now estimate the deviation $\left(1-\beta_{G}\right)$ due to $g_{\text {eff. }}$ In order to do so, the following three assumptions are made:

(1) The average density in the plasma-field mixing layer is roughly half of the density in the bulk plasma:

$$
\langle\rho\rangle \approx \frac{\rho_{\text {core }}}{2}
$$

(2) Consistent with the estimates made earlier, the width of the edge-plasma boundary layer still is at least roughly given by

$$
\delta \approx \frac{c}{\omega_{p i}}=\frac{V_{A}}{\omega_{c i}}
$$

The second part of this equation is an identity. Here, the inner plasma density and the outer magnetic field are used.

(3) A fraction $h$ of the radial fluid velocity is lost by deceleration during the short time increment of interest, $\mathrm{d} t \approx 1 / \omega_{c i}$ and $h \leq 1$. The effective gravitational acceleration $g_{\text {eff }}=-\mathrm{d}\left\langle V_{x}\right\rangle / \mathrm{d} t$, can then be represented as follows. The numerator, $-\mathrm{d}\left\langle V_{x}\right\rangle=h\left\langle V_{x}\right\rangle$, is to be divided by $\mathrm{d} t$, roughly one-fourth of an ion gyroperiod. Then,

$$
g_{\text {eff }} \approx \omega_{c i} h\left\langle V_{x}\right\rangle
$$

Using the above three assumptions and referring to Equation (H7), a simple calculation shows that the deviation of the $\beta_{G}$ from 1 is given by

$$
\frac{\langle\rho\rangle g_{\text {eff }} \delta}{B_{\delta}^{2} / 2 \mu_{0}} \approx h \frac{\left\langle V_{x}\right\rangle}{V_{A}}
$$

Our basic model has been based on the concept of a highly sub-Alfvenic expansion of injected plasma across the flux surfaces, $V_{x} \ll V_{A}$. This approximation corresponds to a very strong vacuum magnetic field and/or injection of propellant at grazing incidence to $\mathbf{B}$, so that the displaced magnetic flux very quickly exerts a backpressure that prevents 
the realization of a large radial expansion velocity. As the plasma expands radially, the plasma pressure falls and the external magnetic pressure rises, so that the net force on the edge layer is inwards. After this initial dynamical adjustment, the plasma may still expand slowly across the magnetic flux, but only by the ions dragging reluctant electrons across the flux. This second phase corresponds to resistive diffusion. Since $h \leq 1$, it then follows that $\beta_{G}$ is very close to 1 . 



\section{Appendix I.-Initial Ratio of Ion Gyroradius to Plasma-Field Interface Width}

Starting with the basic local identity

$$
\frac{a_{i}}{c / \omega_{p i}}=\sqrt{\beta}
$$

we wish to evaluate this quantity first at a representative position in the middle of the plasma's edge gradient layer, the interface region, and then convert that result to the ratio $\left[a_{i}\right]_{\mathrm{mid}} / \delta$, where $\delta$ is the width of the interface; $\delta \approx 2\left[c / \omega_{p} i\right]_{\text {core. }}$ Here, "core" means the internal number density is used to evaluate the ion plasma frequency. In Section 3.1, "Interface Width in Breech of Nozzle" the interface width was estimated to have a somewhat larger value.

When evaluating the local $\beta_{i}=8 \pi n T_{i} / B^{2}$, the local $n$ in the numerator is set to half the core density, and the local magnetic pressure in the denominator is set to half the external magnetic pressure. The result is $\beta_{i}=\beta_{G}$, where the subscript " $G$ " refers to the global ion beta, which is defined by the ratio of core ion pressure to external magnetic pressure. Assuming that the uniform temperatures are equal, $T_{e}=T_{i}$, the total $\beta_{G}$ must be 1 across a static plasma-field mixing layer. Therefore, the ion contribution to $\beta_{G}$ is onehalf; $\beta_{G i}=1 / 2$. Equation (I1) then reads

$$
\left[\left.\frac{a_{i}}{c / \omega_{p i}}\right|_{\text {mid }}=\frac{1}{\sqrt{2}}\right.
$$

However, $\left[\omega_{p i}\right]_{\mathrm{mid}}=\left[\omega_{p i}\right]_{\mathrm{core}} / \sqrt{2}$, when the mid-density is taken as half the core density. Then, Equation (I2) becomes

$$
\frac{\left[r_{i}\right]_{\text {mid }}}{\sqrt{2}\left[c / \omega_{p i}\right]_{\text {core }}}=\frac{1}{\sqrt{2}}
$$

Dividing Equation (I3) by $\sqrt{2}$ and for $\delta \approx 2\left[c / \omega_{p i}\right]_{\text {core }}$, we then obtain

$$
\frac{\left[a_{i}\right]_{\mathrm{mid}}}{\delta}=\frac{1}{2}
$$

Because $\delta$ actually was calculated to have a somewhat larger value, we chose a somewhat smaller final working ratio:

$$
\frac{\left[a_{i}\right]_{\mathrm{mid}}}{\delta}=\frac{1}{3}
$$

The distinction is not overly significant (see Fig. 4). This then characterizes the ratio of electron current-drift velocity to ion thermal velocity at a representative position within the edge-plasma gradient. Of course, different positions within the gradient layer will produce different numerical results for $a_{i} / \delta$. We do not attempt to obtain a profile-dependent result in this report. 



\section{Appendix J.-Generalized Plasma-Field Interface Thickness at the Throat Calculation and Example}

This appendix presents a more generalized derivation of the plasma-field interface thickness at the throat-where both the initial interface thickness at the breech and its growth from breech to throat are taken into account. The related issue of the ion gyroradius to interface thickness ratio equation is also discussed.

\section{J.1 Calculation of Plasma-Field Interface Thickness and Fraction of Attached Plasma Discussion and Example}

Given a desired specific impulse, which is essentially the desired exit velocity $V_{\mathrm{ex}}$ at the effective exit of the thruster's nozzle, the velocity of propellant at the throat is, to a good approximation,

$$
V_{t}=0.5 V_{\mathrm{ex}}
$$

The provisionally estimated travel time $t_{b} t$ of propellant from breech to throat may be expressed as

$$
t_{b t}=\frac{L_{b t}}{V_{t}}
$$

where $L_{b} t$ is the axial distance from breech to throat. This time estimate is subject to correction as described later.

If the interface thickness in the breech were zero, it would thicken with time as

$$
\delta=\sqrt{D t}=\sqrt{\frac{D L_{b t}}{V_{t}}}
$$

so as to acquire this value in the throat, provided that we take beta to be 1 as a representative magnitude within the interface. We provisionally regard this $\delta$ as the width of the radial density profile in the interface, but subject to correction as described later.

If, instead, the boundary layer thickness in the breech were $\delta_{b}$, then the above formula would generalize to

$$
\delta=\sqrt{\delta_{b}^{2}+D t}=\sqrt{\delta_{b}^{2}+\frac{D L_{b t}}{V_{t}}}
$$

This result is based upon the solution of a differential equation in time along the flow.

In this rendering of Equation (J4), a time-integration has been artificially avoided by regarding $D$ as the average value of the resistive diffusivity between breech and throat. However, $D$ does not vary much between breech and throat, whether classical or anomalous due to Lower Hybrid Drift (LHD) microturbulence. Using $D$ at the position of the throat provides a reasonable estimate of the interface thickness for conditions of interest, because the diffusive contribution to Equation (J4) generally does not greatly dominate the initial (breech) contribution $\delta_{b}^{2}$, and moreover, a square root is taken to get the final result.

When modified as described below, Equation (J4) can be utilized to estimate the fraction of attached plasma at the position of the throat.

As shown in Section 3.1, "Interface Width in Breech of Nozzle," the interface thickness in the breech, which appears in Equation (J4), can be estimated as

$$
\delta_{b}=\frac{2 c}{\omega_{p i}}
$$

in which $c$ is the speed of light in free space and $\omega_{p} i$ is the ion plasma frequency.

Equation (J4), moreover, provides the basis for analytic estimates of the ratio of ion gyroradius to interface thickness, $a_{i} / \delta$, describing that ratio as it varies along the flow. That work is presented in Section 3.1. A particular relevance of that ratio is that it plays a central role in analytic theories of LHD microturbulence, and especially in the anomalous magnitude of the associated resistivity. In this regard, it is important to remark that these analytic theories (for example, Refs. 18, 23, 24, and 35) are usually limited to the electrostatic (zero-beta) model, in which the gradient under consideration is only the density gradient. Thus, for the purpose of referring to the seminal analytic results of these theories, the equations for $a_{i} / \delta$ should be viewed within the context of a resistively diffused density gradient. The $\delta$ therein thus represents the density gradient width, without the effect of bidirectional diffusion.

In the application to space vehicle thrusters with magnetic nozzles, however, we need to estimate the fraction of plasma that has become attached to the magnetic nozzle field lines. For that application, inward magnetic diffusion into the plasma is just as important as outward diffusion of plasma into the external magnetic field.

In that case, two corrections ought to be made to Equation (J4). The first is trivial; namely, to expand the travel time by about a factor 2 in order to allow for the time needed to accelerate the interface plasma up to speed. Thus, $t=L_{b} t / V_{t}$ becomes

$$
t=\frac{2 L_{b t}}{V_{t}}
$$


Equation (J6) is the approximate result of an analytic investigation with realistic nozzle geometry for the plasma edge. (This modification with the factor 2 , in principle, should also be made to the above-mentioned equations for the ratio $a_{i} / \delta$, but that will have only a minor effect on the general conclusion that this ratio varies little between breech and throat.)

The second correction is the more subtle, namely, to allow for simultaneous resistive diffusion of plasma into nozzle magnetic field and of field into plasma; the so-called bidirectional diffusion. Each of these diffusive processes separately (in the absence of Hall current and plasma rotation) would accrue a diffusive distance of about $\sqrt{ }(D \mathrm{t})$, starting from zero thickness in the breech. (For $D=3 \mathrm{~m}^{2} / \mathrm{s}$ and $t=10 \mu \mathrm{s}$, this diffusive distance is about $0.5 \mathrm{~cm}$.) Heuristically adding the two effects, we could reasonably estimate the total interface thickness as

$$
\delta=\sqrt{D t}=\sqrt{4 D t}
$$

The estimate that constitutes Equation (J7) is in need of a careful numerical investigation, within the context of a slowly convergent breech-to-throat geometry, as mediated by a continuously distributed and continuously graded magnet coil. Such a gradual configuration geometry would likely preclude the anomalous cross-field loss of plasma observed in a prior resistive MHD simulation, which employed discrete coils and axially limited distances appropriate to an envisioned lab experiment.

Accounting for both of the above-mentioned corrections, Equation (J4) now reads

$$
\delta=\sqrt{\delta_{b}^{2}+\frac{8 D L_{b t}}{V_{t}}}
$$

At sufficiently low temperatures, $D$ would be the classical resistive diffusivity associated with coulomb scattering of electrons on ions.

The classical resistivity itself appears in Equation (200) in CGS units. The coulomb logarithm therein (the logarithm of the ratio of the maximum-to-minimum impact parameter in an electron collision with an ion) has been set to 10 , which is sufficient at the present level of development of our investigation. (See Ref. 9.) Multiplication by $\left(c^{2} / 4 \pi\right)$ converts this resistivity to $D$ in CGS units. Multiplication of the CGS resistivity by $9 \cdot 10^{9}$ converts the resistivity to MKS units. Dividing the latter by $\mu_{0}$, which is the magnetic permeability of free space, then yields $D$ in MKS units. Alternatively, one can convert $D$ (CGS) into $D$ (MKS) by just dividing the former by $10^{4}$.

At sufficiently high temperatures, $D$ could result from LHD microturbulence evolving from edge-gradient instabilities. At intermediate temperatures, $D$ would be appropriately regarded as arising from the sum of both resistivities; that is, the two types of electron collision frequencies, classical and anomalous, would be additive.

We shall now present a numerical example of the calculation of the fraction of attached plasma, based upon Equation (J8). Of course, the parameters to be assumed here can be changed as needed, concomitantly with changes of the resistive diffusivity $D$, and the interface width in the breech $\delta_{b}$

\section{J.2 Calculation of the Fraction of Attached Plasma-An Example}

Consider hydrogen propellant, breech temperature $50 \mathrm{eV}$, and breech number density $1.0 \cdot 10^{15} \mathrm{~cm}^{-3}$. The specific impulse is then about $20000 \mathrm{~s}$, and $V_{t}=1.0 \cdot 10^{7} \mathrm{~cm} / \mathrm{s}$. (Here, we employ approximate numbers, but they can be made more accurate in a final calculation. Of course, other parameters can also be assumed.)

The interface thickness in the breech is then $\delta_{b}=1.7 \mathrm{~cm}$. More to the point,

$$
\delta_{b}^{2}=2.9 \mathrm{~cm}^{2}
$$

For the given parameters, classical resistivity by far dominates over anomalous resistivity due to the LHD instability (see Fig. 4) and one finds approximately

$$
D=3 \cdot 10^{4} \mathrm{~cm} / \mathrm{s}
$$

which is the value in the throat and which thus allows for the temperature drop relative to the breech.

With an axial breech to throat distance of one meter, $L_{b} t=$ $100 \mathrm{~cm}$, then Equation (J8) becomes

$$
\delta=\sqrt{3.0=2.4}=2.3 \mathrm{~cm}
$$

Because we included bidirectional diffusion, the diffusive contribution is about the same as that from the initial (breech) contribution. Without the correction for bidirectional diffusion, the second term would be rather small in comparison to the breech contribution to the interface thickness. That result for density diffusion alone then would be relevant to the gyro-radius ratio to the thickness of the density gradient, as discussed earlier.

In order to calculate the fraction of attached plasma, one must already know the rate of mass ejection, $\dot{m}$. This quantity is supposed to be predetermined by the logistics of the given mission. With a hydrogen plasma radius in the throat, $r_{t}=10 \mathrm{~cm}$, one has $\dot{m}=3.5 \mathrm{~g} / \mathrm{s}$, which already accounts for the drop in density from breech to throat. Different desired values of the mass ejection rate would lead to different values of the plasma radius in the throat, at the assumed breech 
density. (Of course, the plasma radius in the throat must be the result of a self-consistent steady-flow interaction between the injected plasma and the shaped vacuum magnetic nozzle field. Prior simulations have already demonstrated the self-consistent formation of a plasma throat, within the time-dependent resistive MHD model.)

If the core density were to extend throughout the width of the interface, then the fraction of attached plasma would be, to sufficient approximation,

$$
\frac{2 \delta}{r_{t}}=46 \text { percent }
$$

However, the average density in the interface will, of course, be somewhat less than the core density. A reasonable provisional estimate, subject to the results of a future resistive MHD simulation without ill understood anomalies, is that the average density in the interface is roughly half of the core value. (This would be the case for a linear spatial decrease to zero in the radial edge density profile.) Then one finds the fraction of attached plasma to be 23 percent. This is, after all, a significant loss of plasma, which portends that there will be a significant detachment problem to be solved downstream, if the idealized thruster results are to be approached.

At first glance, the above example points to the advantages of working at higher temperatures to slow down resistive diffusion. Then, higher mass propellant ions would be employed to preserve the desired specific impulse. Furthermore, in order to mitigate the initial interface width in the breech, it would be advantageous as well to work with a higher number density of ions, so as to increase the ion plasma frequency there. But note that the higher mass ion decreases the ion plasma frequency, whereas the higher ion charge increases it! The presence of a higher ion charge also tends to increase resistive diffusion due to coulomb scattering of electrons by ions. Obviously, there are several competing effects that need scrutiny. A scenario involving higher stagnation number density and higher stagnation temperature in a propellant consisting of higher mass (and charge) ions, becomes of interest for future investigations. 



\section{Appendix K.-Raleigh-Taylor Instability: Further Considerations}

In this appendix, we furnish the details leading to the spatial dependence of the external magnetic field when flute distortions of the surface are present. In the main report, we presented a derivation of the growth rate of this flute-mode instability in the magnetic nozzle-propellant interface. The derivation given suppressed some details; specifically, the property that the external magnetic field $B$ has a $1 / R$ dependence is crucial, but was not given a full discussion. Here, $R$ is the local longitudinal radius of curvature of the surface, which can be extended beyond the equilibrium surface to signify a spatial variable in the external volume outside of the propellant.

In the equilibrium situation involving only the unperturbed boundary surface of propellant, the above-mentioned spatial dependence of the external magnetic field can readily be demonstrated. The integral form of Ampere's law is employed, using an Ampere circuit around the highly conducting, sharp-boundary propellant configuration, which is assumed to diamagnetically exclude the magnetic flux. Therefore, the integral form of Ampere's law immediately yields the result that $B=\mu_{0} K R_{\mathrm{eq}} / R$, where $R_{\mathrm{eq}}$ is the equilibrium radius of local longitudinal curvature and $K$ is the local transverse surface current per unit longitudinal arc length.

The question remains as to the spatial dependence of the external magnetic field under the nonequilibrium condition associated with the distortion of the surface from $R_{\mathrm{eq}}$ (denoted by $\delta R$ ) into flutes. Then $R=R_{\mathrm{eq}}+\delta R$ represents the distance of a point on the distorted interface from the local center of longitudinal curvature of the interface. (The equilibrium shape of the interface still has longitudinal radius of curvature $R_{\text {eq. }}$ )

Figure 7 illustrates a longitudinal local section of a converging-nozzle surface with "adverse" longitudinal curvature and flutes. The center of longitudinal curvature is at $\mathrm{C}$. The equilibrium surface possesses a radius of longitudinal curvature $R_{\text {eq. }}$. The crests and troughs of the flutes on that surface are indicated by $R=\left(R_{\mathrm{eq}}+\delta R\right)$. The flutes are observed by rotating the r,Z-plane of the figure around the $\mathrm{Z}$-axis, while $\mathrm{C}$ is fixed (in the rotating plane) but the radius of longitudinal curvature fluctuates. The subtended longitudinal angle is $\mathrm{d} \phi$, and the length of arc shown (solid) is $R_{\mathrm{eq}} \mathrm{d} \phi$. In the equilibrium situation, without surface distortions, one may regard $R$ as a radial position variable in the space outside of the unperturbed surface. Since the magnetic field B only exists in the external volume, one may apply the integral form of Ampere's law to the circuit indicated by $(\rightarrow)$, to show that $B=\mu_{0} K\left(R_{\mathrm{eq}} / R\right)$, where $K$ is the surface current per unit longitudinal arc length.

In this appendix we furnish the details leading to the spatial dependence of the external magnetic field when flute distortions of the surface are present. The proof will be carried out primarily verbally and pictorially to facilitate its accessibility, but we believe that it is nevertheless a rigorous proof when based upon the following assumptions and their corollaries:

(1) The propellant core is highly conducting (a "perfect" conductor) and is bounded by a sharply defined surface (the interface) separating the internal field-free plasma from the external longitudinal magnetic nozzle field $\left(B_{r}, B_{Z}\right)$. These are the only components of $\mathbf{B}$ after the original axially symmetric configuration becomes distorted by flute modes.

(2) According to the integral form of Ampere's law, $\nabla \times \mathbf{B}=\mu_{0} \mathbf{J}$, there must necessarily exist a surface current that separates the null-field interior plasma from the nozzle magnetic field in the external region. We denote this surface current density by $\mathbf{K}$.

(3) The longitudinal flute-mode displacement of each point on the sharp-boundary plasma surface occurs only in the $r, Z$-plane corresponding to that equilibrium point and is a periodic function of the azimuthal angle $\theta$. (The cylindrical coordinates are $(r, \theta, Z)$.) We caution that the flute-distortion motion of the surface itself is not identical to the motion of the underlying fluid. Nevertheless, only the surface displacement is relevant to the disposition of the external magnetic field.

(4) Our simple model of the flute distortion is that the local distorted surface remains "parallel" to the equilibrium surface, in the sense depicted in Figure 7(a). This assumption is meant to reproduce, as closely as possible, the fluting of a plane surface, in which the longitudinal generators of the distortions remain parallel to the original plane. This particular model of the flute distortions is realized by having the center of curvature remain fixed in the r,Z-plane as it rotates around the axis of initial symmetry. Then the longitudinal radius of curvature of the surface varies as the plane rotates, thus generating the crests and troughs of the flutes. (Note: Restriction of the present model to address only a short longitudinal increment of the surface means that the phenomena of global ballooning modes are neglected. Localized g-modes are the objects of the present investigation. Eventually, however, the ballooning modes should be studied to provide a more comprehensive picture of processes that affect regions of adverse curvature. Such regions might exist throughout the nozzle: either upstream of the nozzle-throat (where the nozzle narrows down into the throat) or also downstream of the throat (where the diverging field lines are turned so as to straighten out the flow).

(5) The magnetic field must remain external to the distorted fluid surface because magnetic field lines cannot penetrate a perfect conductor if they initially lie outside. Moreover, the field lines must remain longitudinal $\left(B_{\theta}=0\right)$ during the flute distortion, because there exists no conductingsurface motion restricted to longitudinal flutes that can initiate any transverse magnetic field component. (There is 

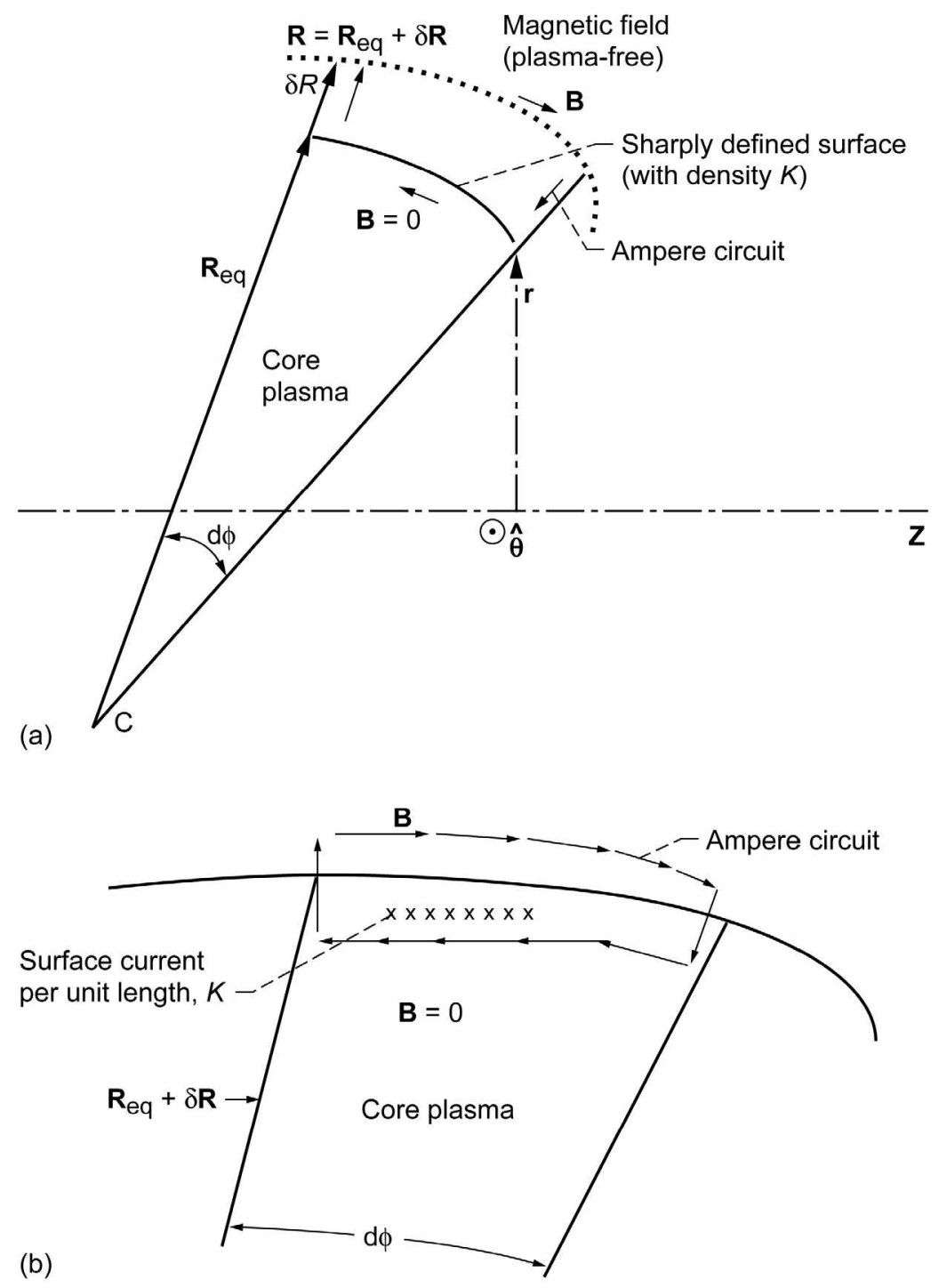

(b)

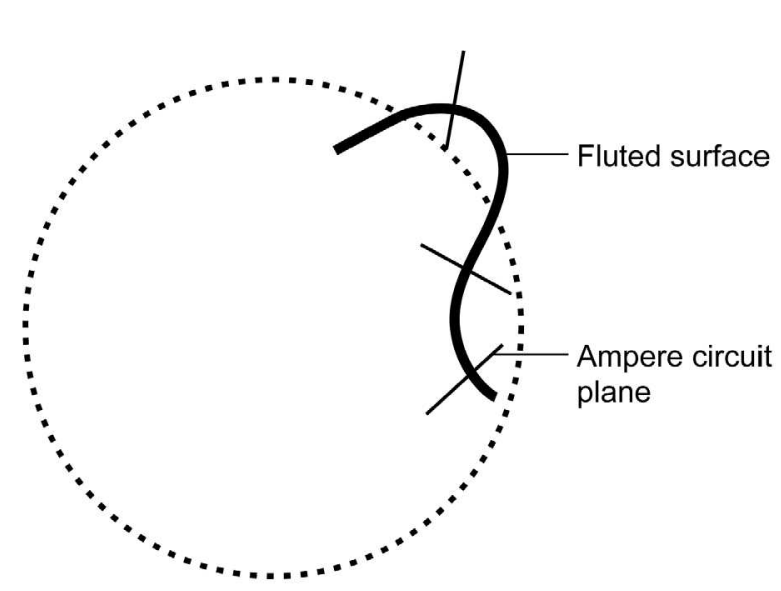

Figure 7.-Ampere circuit planes in region of adverse curvature. (a) Ampere circuit in section with adverse longitudinal curvature and flutes. (b) Ampere circuit with vanishing width. (c) End-on view of fluted surface with three Ampere circuit planes. 
no preferred transverse field direction under the given surface displacement.)

(6) The longitudinal field lines just outside the surface must remain tangential to the distorted surface: first, because they cannot enter or leave the surface and second, because any vacated external vacuum volume would become instantly filled with magnetic field (at the speed of light).

(7) The vector field that describes the current density $\mathbf{K}$ must (of course) lie on the evolving surface during the distortion, and it also must remain locally perpendicular to the contiguous longitudinal magnetic field vectors. Otherwise, if $\mathbf{K}$ had acquired a component along $\mathbf{B}$, then $\mathbf{B}$ would necessarily acquire a transverse component, but this is impossible under the conditions restricted to flute distortions of the surface (see above).

Based upon the above assumptions, we can now show that the magnetic field at the distorted surface varies as $1 / R$, where $R$ is the local longitudinal radius of curvature on the distorted surface. This is done by means of the integral form of Ampere's law.

It can be inferred from Figure 7(a) that as the r,Z-plane is rotated to register a sequence of flute distortions, lines of the vector field $\mathbf{K}$ cannot cross the boundaries of the sector $\mathbf{d} \phi$. For if they did, the surface would then necessarily acquire a component along longitudinal $\mathbf{B}$, which cannot happen in the considered flute distortions (see above). Therefore, the total surface current in $\mathrm{d} \phi$ is conserved. That is, $K R \mathrm{~d} \phi$ is constant. ( $R \mathrm{~d} \phi$ is the longitudinal arc length crossed by the surface current.)
Figure 7(b) shows an Ampere circuit of vanishing width, indicated by $(\rightarrow)$. The thin Ampere circuit contains the propellant's sharply defined surface. The model considered here is delimited by a longitudinal angle increment $d \phi$, which is measured from the center of longitudinal curvature C shown in Figure 7(a). The symbol "xxxxx" schematically indicates the surface current per unit longitudinal length, denoted as $K$. The plane of Figure 7(b) (and 7(a)) is the $r, Z$-plane, but the plane of the Ampere circuit is perpendicular to the distorted (fluted) surface. Hence the Ampere circuit is in the r,Z-plane only at the crests and troughs of the distorted surface. Figure 7(c) illustrates this end-on view of a fluted surface, showing three Ampere circuit planes.

Considering Figure 7(b), we can apply the integral form of Ampere's law to the indicated circuit. The result reads $B R$ $\mathrm{d} \phi=\mu_{0} K R \mathrm{~d} \phi$; also, $B R \mathrm{~d} \phi=$ constant. Hence, B varies as $1 / R$ even when the surface possesses flute distortions. This result was specifically utilized in Section 4.2.1, "Surface Instability of Static Plasma in Region of Adverse Curvature," in the discussion of the exponential growth rate of the linearized flute-mode fluctuations.

Aside from the spatial variation of magnetic field $\mathbf{B}$ near the surface of the propellant, there is a concern that $\mathbf{B}$ could suffer an overall shift because of a change in surface current $\mathbf{K}$ as the flutes evolve. However, the large inductance of the global currents provides very large impedance against shifts in $\mathbf{K}$ on the time scales for growth of the Raleigh-Taylor instabilities. 



\section{References}

1. Mikellides, I.G., et al.: Theoretical Design of a MagneticNozzle Acceleration System for Fusion Propulsion. AIAA2000-3367, 2000.

2. Turchi, P.J., et al.: Numerical Simulation of Magnetic Nozzle Flow for Nuclear-Fusion Space Propulsion. AIAA-19992701, 1999.

3. Mikellides, I.G., et al.: Design of a Fusion Propulsion SystemPart 2: Numerical Simulation of Magnetic-Nozzle Flows. J. Propul. P., vol. 18, no. 1, 2002.

4 Williams, Craig H., et al.: Realizing "2001: A Space Odyssey"-Piloted Spherical Torus Nuclear Fusion Propulsion. NASA/TM-2005-213559, 2001.

5. Williams, Craig H., et al:: A Sperical Torus Nuclear Fusion Reactor Space Propulsion Vehicle Concept for Fast Interplanetary Piloted and Robotic Missions. AIAA-1999-2704, 1999.

6. Gerwin, Richard A., et al.: Characterization of Plasma Flow Through Magnetic Nozzles. AL-TR-89-092 (LA-UR-894212), 1989.

7. Moses, R.W.J.; Gerwin, R.A.; and Schoenberg, K.F.: Resistive Plasma Detachment in Nozzle Based Coaxial Thrusters. AIP Conference Proceedings, pt. 3, 1992, pp. 1293-1303.

8. Peter Turchi, 1999, Ohio State University, Columbus, $\mathrm{OH}$, personal communication.

9. Braginskii, S.I.: Transport Processes in a Plasma. Rev. Plasma Phys., vol. I, M.A. Leontovich, ed., Consultants Bureau, New York, NY, 1965, p. 205.

10. Spitzer, Lyman: Physics of Fully Ionized Gases. Interscience Publishers, New York, NY, 1956.

11. Black, D.C., et al.: Two-Dimensional Magnetic Field Evolution Measurements and Plasma Flow Speed Estimates From the Coaxial Thruster. Phys. Plasmas, vol. 1, issue 9, 1994, pp. 3115-3131.

12. Sgro, A.G.; and Nielson, C.W.: Hybrid Model Studies of Ion Dynamics and Magnetic Field Diffusion During Pinch Implosions. Phys. Fluids, vol. 19, 1976, pp. 126-133.

13. Chodura, R.: A Hybrid Fluid-Particle Model of Ion-Heating in High Mach Number Shock Waves. Nuclear Fusion Journal, vol. 15 , no. 7,1975, p. 55.

14. Brackbill, J.U., et al.: Nonlinear Evolution of the Lower-Hybrid Drift Instability. Phys. Fluids, vol. 27, no. 11, 1984, pp. 26822693.

15. Drake, J.F.; Huba, J.D.; and Gladd, N.T.: Stabilization of the Lower-Hybrid-Drift Instability in Finite-Beta Plasmas. Phys Fluids, vol. 26, no. 8, 1983, pp. 2247-2249.

16. Peter, W.; Ron, A.; and Rostoker, N.: Instability of the Boundary-Layer Between a Streaming Plasma and a Vacuum Magnetic-Field. Phys. Fluids, vol. 26, no. 8, 1983, pp. 2276-2280.

17. Ripin, B.H., et al.: Sub-Alfvenic Plasma Expansion. Phys. Fluids B, vol. 5, no. 10, 1993, pp. 3491-3506.

18. Davidson, R.C.; and Gladd, N.T.: Anomalous Transport Properties Associated With Lower-Hybrid-Drift Instability. Phys. Fluids, vol. 18, no. 10, 1975, pp. 1327-1335.

19. Freidberg, J.P.; and Gerwin, R.A.: Lower Hybrid Drift Instability at Low Drift Velocities. Phys. Fluids, vol. 20, no. 8, 1977, pp. 1311-1315.

20. Gladd, N.T.; Sgro, A.G.; and Hewett, D.W.: Microstability Properties of the Sheath Region of a Field-Reversed Configuration. Phys. Fluids, vol. 28, no. 7, 1985, pp. 2222-2234.
21. Choueiri, E.Y.: Electron-Ion Streaming Instabilities of an Electromagnetically Accelerated Plasma. Ph.D. Thesis, Princeton Univ., 1991.

22. Huba, J.D.; and Ossakow, S.L.: Destruction of Cyclotron Resonances in Weakly Collisional, Inhomogeneous Plasmas. Phys. Fluids, vol. 22, no. 7, 1979, pp. 1349-1354.

23. Davidson, R.C.: Quasilinear Stabilization of Lower-HybridDrift Instability. Phys. Fluids, vol. 21, no. 8, 1978, pp. 13751380.

24. Gary, S. Peter: Wave-Particle Transport From Electrostatic Instabilities. Phys. Fluids, vol. 23, issue 6, 1980, pp. 1193-1204.

25. Drake, J.F.; Guzdar, P.N.; and Huba, J.D.: Saturation of the Lower-Hybrid-Drift Instability by Mode-Coupling. Phys. Fluids, vol. 26, no. 3, 1983, pp. 601-604.

26. Drake, J.F., et al.: Nonlinear Mode-Coupling Theory of the Lower-Hybrid-Drift Instability. Phys. Fluids, vol. 27, no. 5, 1984, pp. 1148-1159.

27. Fahrbach, H.U., et al.: Measurement of Lower Hybrid Drift Fluctuations in the Boundary-Layer of a High-Beta Plasma by Collective $\mathrm{CO}_{2}$-Laser Light-Scattering. Nucl. Fusion, vol. 21, no. 2,1981 , pp. 257-270.

28. Berk, H.L.; and Dominguez, R.R.: Variational Method for Electromagnetic Waves in a Magneto-Plasma. J. Plas. Phys., vol. 18,1977 , pp. $31-48$.

29. Hastings, D.E.; and McCune, J.E.: The High- $\beta$ Universal Drift Mode. Phys. Fluids, vol. 25, 1982, pp. 509-517.

30. Huba, J.D.; and Gary, S.P.: Finite- $\beta$ Stabilization of the Universal Drift Instability: Revisited. Phys. Fluids, vol. 25, 1982, pp. 1821-1823.

31. Lapenta, G.; and Brackbill, J.U.: 3D Reconnection Due to Oblique Modes: A Simulation of Harris Current Sheets. Nonlinear Processes in Geophysics, vol. 7, no. 3/4, 2000, pp. 151-158.

32. Huba, J.D.: Finite Larmor Radius Magnetohydrodynamics of the Rayleigh-Taylor Instability. Phys. Plasmas, vol. 3, no. 7, 1996, pp. 2523-2532.

33. Huba, J.D.: Hall Magnetohydrodynamics In-Space and Laboratory Plasmas. Phys. Plasmas, vol. 2, no. 6, pt. 2, 1995 , pp. 2504-2513.

34. McKenna, K.F., et al.: Radial Plasma Structure During a ThetaPinch Implosion -Flute Instabilities. Phys. Fluids, vol. 18, no. 10, 1975, pp. 1371-1373.

35. Gary, S. Peter; and Thomsen, Michelle F.: Collisionless Electrostatic Interchange Instabilities. J. Plasma Phys., vol. 28, pt. 3, 1982, pp. 551-564.

36. Nagornyj, V.P.; Ryutov, D.D.; and Stupakov, G.V.: Flute Instability of Plasma in a Gas-Dynamic Trap. Nucl. Fusion, vol. 24, no. 11, 1984, pp. 1421-1438.

37. Landau, L.D.; and Lifshitz, E.M.: Fluid Mechanics. Second ed., Pergamon Books Ltd., Oxford, 1987.

38. Huba, J.D.; and Winske, D.: Rayleigh-Taylor Instability: Comparison of Hybrid and Nonideal Magnetohydrodynamic Simulations. Phys. Plasmas, vol. 5, no. 6, 1998, pp. 2305-2316.

39. Migliuolo, S.: Stability Theory of Drift-Type Flute Modes in Finite-Beta Plasmas. Phys. Fluids, vol. 25, no. 12, 1982 , pp. 2289-2294.

40. Freidberg, J.P.; and Wesson, J.A.: Ion Drift Wave Instability. Phys. Fluids, vol. 13, issue 4, 1970, pp. 1009-1016. 


\begin{tabular}{|c|c|c|}
\hline \multicolumn{2}{|c|}{ REPORT DOCUMENTATION PAGE } & $\begin{array}{l}\text { Form Approved } \\
\text { OMB No. 0704-0188 }\end{array}$ \\
\hline \multicolumn{3}{|c|}{ 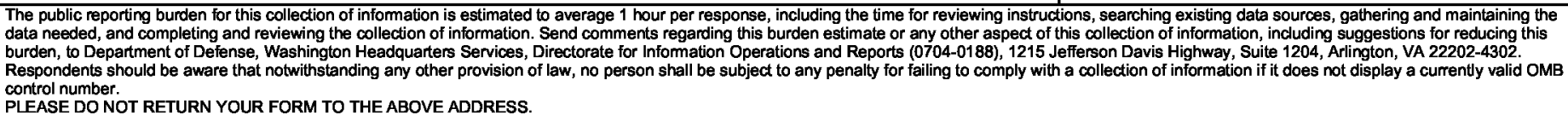 } \\
\hline $\begin{array}{l}\text { 1. REPORT DATE (DD-MM-YYYY) } \\
01-12-2009\end{array}$ & $\begin{array}{l}\text { 2. REPORT TYPE } \\
\text { Technical Paper }\end{array}$ & 3. DATES COVERED (From - To) \\
\hline \multirow{3}{*}{\multicolumn{2}{|c|}{$\begin{array}{l}\text { 4. TITLE AND SUBTITLE } \\
\text { Integrity of the Plasma Magnetic Nozzle }\end{array}$}} & $\begin{array}{l}\text { 5a. CONTRACT NUMBER } \\
\text { C-32057-G }\end{array}$ \\
\hline & & 5b. GRANT NUMBER \\
\hline & & 5c. PROGRAM ELEMENT NUMBER \\
\hline \multirow{3}{*}{\multicolumn{2}{|c|}{$\begin{array}{l}\text { 6. AUTHOR(S) } \\
\text { Gerwin, Richard, A. }\end{array}$}} & 5d. PROJECT NUMBER \\
\hline & & 5e. TASK NUMBER \\
\hline & & $\begin{array}{l}\text { 5f. WORK UNIT NUMBER } \\
\text { WBS 22-973-90-01 }\end{array}$ \\
\hline \multicolumn{2}{|c|}{$\begin{array}{l}\text { 7. PERFORMING ORGANIZATION NAME(S) AND ADDRESS(ES) } \\
\text { National Aeronautics and Space Administration } \\
\text { John H. Glenn Research Center at Lewis Field } \\
\text { Cleveland, Ohio 44135-3191 }\end{array}$} & $\begin{array}{l}\text { 8. PERFORMING ORGANIZATION } \\
\text { REPORT NUMBER } \\
\text { E-14974 }\end{array}$ \\
\hline \multirow{2}{*}{\multicolumn{2}{|c|}{$\begin{array}{l}\text { 9. SPONSORING/MONITORING AGENCY NAME(S) AND ADDRESS(ES) } \\
\text { National Aeronautics and Space Administration } \\
\text { Washington, DC 20546-0001 }\end{array}$}} & $\begin{array}{l}\text { 10. SPONSORING/MONITOR'S } \\
\text { ACRONYM(S) } \\
\text { NASA }\end{array}$ \\
\hline & & $\begin{array}{l}\text { 11. SPONSORING/MONITORING } \\
\text { REPORT NUMBER } \\
\text { NASA/TP-2009-213439 }\end{array}$ \\
\hline \multicolumn{3}{|c|}{$\begin{array}{l}\text { 12. DISTRIBUTION/AVAILABILITY STATEMENT } \\
\text { Unclassified-Unlimited } \\
\text { Subject Category: } 15 \\
\text { Available electronically at http://gltrs.grc.nasa.gov } \\
\text { This publication is available from the NASA Center for AeroSpace Information, 443-757-5802 }\end{array}$} \\
\hline
\end{tabular}

\section{SUPPLEMENTARY NOTES}

\section{ABSTRACT}

This report examines the physics governing certain aspects of plasma propellant flow through a magnetic nozzle, specifically the integrity of the interface between the plasma and the nozzle's magnetic field. The injection of $100 \mathrm{~s}$ of eV plasma into a magnetic flux nozzle that converts thermal energy into directed thrust is fundamental to enabling $10000 \mathrm{~s}$ of seconds specific impulse and $10 \mathrm{~s}$ of $\mathrm{kW} / \mathrm{kg}$ specific power piloted interplanetary propulsion. An expression for the initial thickness of the interface is derived and found to be $\sim 10^{-2} \mathrm{~m}$. An algorithm is reviewed and applied to compare classical resistivity to gradient-driven microturbulent (anomalous) resistivity, in terms of the spatial rate and time integral of resistive interface broadening, which can then be related to the geometry of the nozzle. An algorithm characterizing plasma temperature, density, and velocity dependencies is derived and found to be comparable to classical resistivity at local plasma temperatures of $\sim 200 \mathrm{eV}$. Macroscopic flute-mode instabilities in regions of "adverse magnetic curvature" are discussed; a growth rate formula is derived and found to be one to two e-foldings of the most unstable Rayleigh-Taylor (RT) mode. After establishing the necessity of incorporating the Hall effect into Ohm's law (allowing full Hall current to flow and concomitant plasma rotation), a critical nozzle length expression is derived in which the interface thickness is limited to about 1 ion gyroradius.

\section{SUBJECT TERMS}

Magnetic nozzle; Nuclear fusion; Space propulsion; Plasma physics

\begin{tabular}{|c|c|c|c|c|c|}
\hline \multicolumn{3}{|c|}{ 16. SECURITY CLASSIFICATION OF: } & \multirow{2}{*}{$\begin{array}{l}\text { 17. LIMITATION OF } \\
\text { ABSTRACT } \\
\text { UU }\end{array}$} & \multirow{2}{*}{$\begin{array}{l}\text { 18. NUMBER } \\
\text { OF } \\
\text { PAGES } \\
102\end{array}$} & $\begin{array}{l}\text { 19a. NAME OF RESPONSIBLE PERSON } \\
\text { STI Help Desk (email:help@sti.nasa.gov) } \\
\text { 19b. TELEPHONE NUMBER (include area code) }\end{array}$ \\
\hline $\begin{array}{l}\text { a. REPORT } \\
\text { U }\end{array}$ & $\begin{array}{l}\text { b. ABSTRACT } \\
\text { U }\end{array}$ & $\begin{array}{l}\text { c. THIS } \\
\text { PAGE } \\
\text { U }\end{array}$ & & & $\begin{array}{l}\text { 19b. TELEPHONE NUMBER (include area code) } \\
443-757-5802\end{array}$ \\
\hline
\end{tabular}



